\title{
THE SPECTRAL SEQUENCE RELATING ALGEBRAIC $K$-THEORY TO MOTIVIC COHOMOLOGY
}

\author{
BY ERIC M. FRIEDLANDER ${ }^{1}$ AND ANDREI SUSLIN ${ }^{2}$
}

\begin{abstract}
Beginning with the Bloch-Lichtenbaum exact couple relating the motivic cohomology of a field $F$ to the algebraic $K$-theory of $F$, the authors construct a spectral sequence for any smooth scheme $X$ over $F$ whose $E_{2}$ term is the motivic cohomology of $X$ and whose abutment is the Quillen $K$-theory of $X$. A multiplicative structure is exhibited on this spectral sequence. The spectral sequence is that associated to a tower of spectra determined by consideration of the filtration of coherent sheaves on $X$ by codimension of support.
\end{abstract}

( 2002 Éditions scientifiques et médicales Elsevier SAS

RÉSUMÉ. - Partant du couple exact de Bloch-Lichtenbaum, reliant la cohomologie motivique d'un corps $F$ à sa $K$-théorie algébrique, on construit, pour tout schéma lisse $X$ sur $F$, une suite spectrale dont le terme $E_{2}$ est la cohomologie motivique de $X$ et dont l'aboutissement est la $K$-théorie de Quillen de $X$. Cette suite spectrale, qui possède une structure multiplicative, est associée à une tour de spectres déterminée par la considération de la filtration des faisceaux cohérents sur $X$ par la codimension du support.

(C) 2002 Éditions scientifiques et médicales Elsevier SAS

The purpose of this paper is to establish in Theorem 13.6 a spectral sequence from the motivic cohomology of a smooth variety $X$ over a field $F$ to the algebraic $K$-theory of $X$ :

$$
E_{2}^{p, q}=H^{p-q}(X, \mathbb{Z}(-q))=C H^{-q}(X,-p-q) \quad \Rightarrow \quad K_{-p-q}(X) .
$$

Such a spectral sequence was conjectured by Beilinson [2] as a natural analogue of the AtiyahHirzebruch spectral sequence from the singular cohomology to the topological $K$-theory of a topological space. The expectation of such a spectral sequence has provided much of the impetus for the development of motivic cohomology (e.g., $[3,34]$ ) and should facilitate many computations in algebraic $K$-theory.

In the special case in which $X$ equals $\operatorname{Spec} F$, this spectral sequence was established by Bloch and Lichtenbaum [5]. Our construction depends crucially upon the main result of [5], the existence of an exact couple relating the motivic cohomology of the field $F$ to the multirelative $K$-theory of coherent sheaves on standard simplices over $F$ (recalled as Theorem 5.5 below). A major step in generalizing the work of Bloch and Lichtenbaum is our re-interpretation of their spectral sequence in terms of the "topological filtration" on the $K$-theory of the standard cosimplicial scheme $\Delta^{\bullet}$ over $F$. We find that the spectral sequence arises from a tower of

\footnotetext{
${ }^{1}$ Partially supported by the N.S.F. and the N.S.A.

${ }^{2}$ Partially supported by the N.S.F. grant DMS-9510242.
} 
$\Omega$-prespectra

$$
\mathcal{K}\left(\Delta^{\bullet}\right)=\mathcal{K}^{\mathcal{C}^{0}}\left(\Delta^{\bullet}\right) \leftarrow \mathcal{K}^{\mathcal{C}^{1}}\left(\Delta^{\bullet}\right) \leftarrow \mathcal{K}^{\mathcal{C}^{2}}\left(\Delta^{\bullet}\right) \leftarrow \cdots
$$

Thus, even in the special case in which $X$ equals $\operatorname{Spec} F$, we obtain a much clearer understanding of the Bloch-Lichtenbaum spectral sequence which is essential for purposes of generalization.

Following this re-interpretation, we proceed using techniques introduced by Voevodsky in his study of motivic cohomology. In order to do this, we provide an equivalent formulation of $K$-theory spectra associated to coherent sheaves on $X$ with conditions on their supports which is functorial in $X$. We then verify that the homotopy groups of these spectra satisfy almost all of the conditions of a pretheory in the sense of Voevodsky. This enables us to apply Voevodsky's machinery to identify the homotopy fibers of the tower for $\mathcal{K}\left(\Delta^{\bullet} \times X\right)$ for $X$ the spectrum of a semi-local $F$-algebra. We then globalize this result to an arbitrary smooth, quasiprojective variety $X$ over $F$ using Brown-Gersten techniques for simplicial sheaves. (In [16], Levine constructs the spectral sequence (13.6.1) by starting with our results for Spec $F$ and then proceeding by alternate methods.)

We conclude this introduction with a brief summary of the various sections of this paper. The first four sections of the paper are dedicated to proving for a simplicial prespectrum $X_{\bullet}$ that the homotopy fiber $f i b\left(c u b e_{n}\left(X_{\bullet}\right)\right)$ of the associated $n$-cube of prespectra maps naturally to the $(n-1)$-st loops $\Omega^{n-1}\left|X_{\bullet}\right|$ of the geometric realization inducing an isomorphism in homotopy groups in a specified range of degrees. The relevance of this purely topological result for our purposes is that the multirelative $K$-theory considered by Bloch and Lichtenbaum is easily identified as the homotopy groups of such a homotopy fiber. The proof of this general topological result for simplicial prespectra proceeds in several steps. In Section 2, we show the existence of a natural map from the homotopy cofiber $\operatorname{cofib}\left(\operatorname{cube}_{n}\left(X_{\bullet}\right)\right)$ to $\Sigma\left|X_{\bullet}\right|$ which induces a homotopy equivalence in a specified range. This is proved using the special case of a simplicial abelian group considered in Section 1. Sections 3 and 4 then present a comparison of (iterated) homotopy fibers and cofibers for maps of prespectra.

In Theorem 6.2, we present our topological interpretation of the Bloch-Lichtenbaum exact couple (for $X$ equal to Spec $F$ ). Having observed in Theorem 5.7 that the derived exact couple of the Bloch-Lichtenbaum exact couple has a pleasing interpretation in terms of $K$-theory of cosimplicial schemes, the verification of this topological interpretation is relatively straightforward. Section 7 establishes the homotopy invariance of the $K$-theory prespectra $\mathcal{K}^{\mathcal{C}^{q}}\left(\Delta^{\bullet} \times X\right)$ which is required for our modification of the $K$-theory spectra $\mathcal{K}^{\mathcal{C}^{q}}\left(\Delta^{\bullet} \times X\right)$ given in the following section. This modification in the case $X=\operatorname{Spec} F$, replacing coherent sheaves with support of codimension $\geqslant q$ on $\Delta^{n}$ by coherent sheaves on $\Delta^{n} \times \mathbb{A}^{q}$ with support quasi-finite over $\Delta^{n}$, is shown in Theorem 8.6 to yield a prespectrum $\mathcal{K}^{\mathcal{Q}, \mathbb{A}^{q}}\left(\Delta^{\bullet}\right)$ weakly equivalent to $\mathcal{K}^{\mathcal{C}^{q}}\left(\Delta^{\bullet}\right)$. Section 10 introduces "pseudo pretheories", a slightly less rigid structure than Voevodsky's pretheories and Corollary 11.4 shows that our modified prespectra yielding $K$-theory with support conditions determine pseudo pretheories.

The work of earlier sections establishes the necessary tower of fibrations for $X$ equal to Spec $R$ where $R$ is the semi-local ring associated to a finite collection of points on a smooth scheme $X$ of finite type over $F$. After stipulating our formulation of motivic cohomology of smooth schemes in Section 12, we employ in Section 13 the techniques of simplicial sheaves developed by Brown and Gersten [9] to globalize the requisite tower of fibrations and thereby the Bloch-Lichtenbaum spectral sequence. We observe in Theorem 13.12 that our spectral sequence for smooth varieties easily yields a similar spectral sequence for an arbitrary scheme $X$ of finite type over $F$ (converging to the $K$-theory $K_{*}^{\prime}(X)$ of coherent sheaves on $X$ ). After discussing multiplicative structures on exact couples and spectral sequences in Section 14, we establish in Section 15 the expected natural multiplicative structure for the spectral sequence for smooth varieties. Finally, in 
Section 16, we observe that our spectral sequence yields similar multiplicative spectral sequences for $K$-theory with finite or rational coefficients.

In five apendices, we discuss products in cohomology of unbounded complexes, prespectra and $\Omega$-prespectra, constructions leading to a functorial definition of $K$-theory, the Brown-Gersten techniques for simplicial sheaves, and closed cofibrations of topological spaces.

\section{Iterated cofibers for simplicial abelian groups}

In this first section, we investigate the relationship between the total complex of an $n$-cube cube $_{n}\left(A_{\bullet}\right)$ of abelian groups associated to a simplicial abelian group $A_{\bullet}$ with the associated chain complex of $A_{\text {. }}$. Our conclusion in Theorem 1.2 is that there is a natural quasi-isomorphism from $\operatorname{Tot}\left(\operatorname{cube}_{n}\left\{A_{\bullet}\right\}\right)$ to $\left(\sigma_{\leqslant n-1}\left(\mathcal{M}\left(A_{\bullet}\right)\right)[1]\right.$, the naive truncation of the Moore complex of $A_{\bullet}$ shifted "to the left". The refinement of this to simplicial spaces in the next section will provide the key link between multirelative $K$-groups (defined in terms of the homotopy fibre of a multi-cube associated to applying the $K$-functor to a cosimplicial variety) and $K$-groups of the cosimplicial variety (defined as an associated total space).

By an $n$-cube in a category $\mathcal{C}$ we mean a commutative diagram in $\mathcal{C}$ indexed by vertices (and edges) of an $n$-dimensional cube. To be more precise, an $n$-cube $Y_{\bullet}, \ldots, \bullet$ in $\mathcal{C}$ consists of the data of objects $Y_{i_{0}, \ldots, i_{n-1}} \in \mathcal{C}$ (for each $n$-tuple of indices $\left(i_{0}, \ldots, i_{n-1}\right) \in\{0,1\}^{\times n}$ ) and the data of arrows

$$
d_{k}=d_{k}^{i_{0}, \ldots, i_{k-1}, i_{k+1}, \ldots, i_{n-1}}: Y_{i_{0}, \ldots, 1, \ldots, i_{n-1}} \rightarrow \underset{k}{i_{0}, \ldots, 0, \ldots, i_{n-1}},
$$

such that for $k<l$ all diagrams of the form

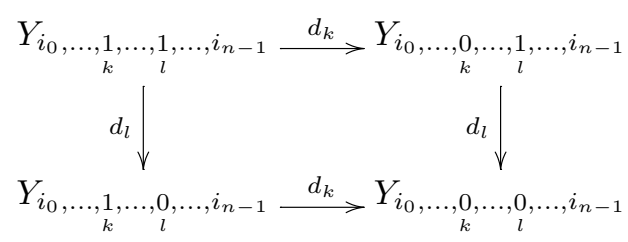

commute. The same concept may be described in slightly different terms. Note that to give an $n$-tuple of indices $\left(i_{0}, \ldots, i_{n-1}\right) \in\{0,1\}^{\times n}$ is the same as to give a subset $S=\left\{k: i_{k}=1\right\}$ of $[n-1]=\{0,1, \ldots, n-1\}$. One checks immediately that the $n$-cube $Y_{\bullet}, \ldots, \bullet$ is nothing but a contravariant functor to $\mathcal{C}$ from the category whose objects are subsets $S \subset\{0,1, \ldots, n-1\}$ and whose maps $S \rightarrow T$ are inclusions. We often use the notation $\partial_{l}: Y_{S} \rightarrow Y_{T}$ for the structure morphism corresponding to the embedding of the subset $T=\left\{s_{0}, \ldots, \hat{s}_{l}, \ldots, s_{k-1}\right\}$ into the set $S=\left\{s_{0}<\cdots<s_{l}<\cdots<s_{k-1}\right\}$ (thus $\partial_{l}=d_{s_{l}}$ ).

To any $n$-cube $Y=Y_{\bullet} \ldots \bullet$ one can associate two $(n-1)$-cubes, which we usually denote $Y_{1}$ and $Y_{0}$. These cubes are defined via the formulas

$$
\left(Y_{1}\right)_{i_{0}, \ldots, i_{n-2}}=Y_{i_{0}, \ldots, i_{n-2}, 1} \quad\left(Y_{0}\right)_{i_{0}, \ldots, i_{n-2}}=Y_{i_{0}, \ldots, i_{n-2}, 0}
$$

Assume that the category $\mathcal{C}$ has a final object $*$ and let $X \bullet$ be a simplicial object in $\mathcal{C}$. For any $n \geqslant 0$ we define a $n$-cube $Y=$ cube $_{n}\left(X_{\bullet}\right)$ setting

$$
Y_{i_{0}, \ldots, i_{n-1}}=X_{i_{0}+\cdots+i_{n-1}-1}
$$


(here we use convention $X_{-1}=*$ ) and taking the map

$$
d_{k}: Y_{i_{0}, \ldots, 1, \ldots, i_{n-1}} \rightarrow Y_{i_{0}, \ldots, 0, \ldots, i_{n-1}}
$$

to be $\partial_{i_{0}+\cdots+i_{k-1}}$. Note that with these definitions the arrow $\partial_{l}: Y_{S}=X_{|S|-1} \rightarrow Y_{T}=X_{|S|-2}$ as defined above coincides with the face operator $\partial_{l}$ of the original simplicial object $X$. . Another useful general remark is that for any $n$ we have a natural identification

$$
\left\{\operatorname{cube}_{n}\left(X_{\bullet}\right)\right\}_{0}=\text { cube }_{n-1}\left(X_{\bullet}\right) .
$$

We start with the following auxiliary construction. Let $r<n$ be a pair of integers. We consider partially defined non-decreasing surjective maps $\phi:[n-1] \rightarrow[r]$ with domain of definition $\operatorname{Dom}(\phi)$ consisting of $r+k+1$ elements. Denote by $C_{k}=C_{k}(n, r)$ the free abelian group generated by such maps. Define the differential $d: C_{k} \rightarrow C_{k-1}$ by the formula

$$
d(\phi)=\sum_{i=1}^{r+k+1}(-1)^{i-1} \partial_{i-1}(\phi),
$$

where $\operatorname{Dom}\left(\partial_{i-1}(\phi)\right)$ is obtained from $\operatorname{Dom}(\phi)$ by deleting the $i$ th element, and $\partial_{i-1}(\phi)$ is the restriction of $\phi$ to $\operatorname{Dom}\left(\partial_{i-1}(\phi)\right)$ in case this restriction is surjective and $\partial_{i-1}(\phi)=0$ otherwise. One checks easily that in this way we get a complex

$$
C_{\bullet}=\left(C_{0} \leftarrow C_{1} \leftarrow \cdots \leftarrow C_{n-r-1}\right) .
$$

Lemma 1.1.- The obvious augmentation map $C_{0} \rightarrow \mathbb{Z}$ defines a quasi-isomorphism $C_{\bullet}=C_{\bullet}(n, r) \rightarrow \mathbb{Z}$.

Proof. - We proceed by induction on $r$. In case $r=0$ our complex coincides with the standard complex computing homology of a $n-1$ simplex and the statement is obvious. Denote by $C_{\bullet}^{0} \subset C_{\bullet}$ the subcomplex generated by those functions $\phi$ for which $\phi^{-1}(0)=\{0\}$. This subcomplex is canonically isomorphic to $C_{\bullet}(n-1, r-1)$ and hence is a resolution of $\mathbb{Z}$ according to the induction hypothesis. Define further a homotopy operator $s: C_{k} \rightarrow C_{k+1}$ via the formula

$$
s(\phi)= \begin{cases}0 & \text { if } 0 \in \operatorname{Dom}(\phi), \\ \text { the unique extension of } \phi \text { to } 0 \cup \operatorname{Dom}(\phi) & \text { if } 0 \notin \operatorname{Dom}(\phi) .\end{cases}
$$

A straightforward verification shows that the operator $p=1-d s-s d$ is given by the formula

$$
p(\phi)= \begin{cases}0 & \text { if }\left|\phi^{-1}(0)\right|>1, \\ \phi_{0} & \text { if }\left|\phi^{-1}(0)\right|=1 .\end{cases}
$$

Here $\operatorname{Dom}\left(\phi_{0}\right)=\left(\operatorname{Dom}(\phi) \backslash \phi^{-1}(0)\right) \cup\{0\}$ and $\phi_{0}$ coincides with $\phi$ on the set $\operatorname{Dom}(\phi) \backslash \phi^{-1}(0)$ whereas $\phi_{0}(0)=0$ (in particular $\phi_{0}=\phi$ in case $\phi^{-1}(0)=\{0\}$ ). Thus $p$ defines a homomorphism of complexes $p: C_{\bullet} \rightarrow C_{\bullet}^{0}$ which is inverse (up to homotopy) to the obvious embed$\operatorname{ding} i: C_{\bullet}^{0} \hookrightarrow C_{\bullet}$. This shows that the embedding $i$ is a quasi-isomorphism and concludes the proof.

Recall (see [20]) that the category of simplicial abelian groups is naturally equivalent to the category of non-negative complexes of degree -1 . The complex corresponding to the simplicial 
abelian group $A_{\bullet}$ is its Moore complex

$$
\mathcal{M}\left(A_{\bullet}\right)=\left(M_{o} \stackrel{\partial_{0}}{\leftarrow} M_{1} \stackrel{\partial_{0}}{\longleftarrow} M_{2} \leftarrow \cdots\right)
$$

Here $M_{n} \subset A_{n}$ is the intersection of kernels of the face operations $\partial_{i}: A_{n} \rightarrow A_{n-1}(i=1, \ldots, n)$ and the differential of the Moore complex coincides with the face operation $\partial_{0}$. We shall use also a slightly different description of the complex $\mathcal{M}\left(A_{\bullet}\right)$ : namely, the complex $\mathcal{M}\left(A_{\bullet}\right)$ is naturally isomorphic to the quotient of the complex obtained from $A_{\bullet}$ by taking the alternating sum of all face operations as a differential (we keep the same notation $A$ • for this complex) modulo the subcomplex consisting of degenerate elements.

To each simplicial abelian group $A_{\bullet}$ (and each $n \geqslant 0$ ) we may associate the $n$-cube in the category $A b:$ cube $_{n}\left\{A_{\bullet}\right\} \equiv Y_{\bullet}, \ldots, \bullet$. Note further that $n$-cubes in the category $A b$ are the same as $n$-complexes bounded in all directions between 0 and 1 . Thus $Y_{\bullet}, \ldots, \bullet$ may be viewed as a $n$-complex. Denote by $T_{\bullet}=\operatorname{Tot}\left(Y_{\bullet}, \ldots, \bullet\right)$ the corresponding total complex.

For a complex $A_{*}$ of abelian groups with differential of degree -1 (i.e., a homological complex), we define the complex $A[k]$, setting $A[k]_{n}=A_{n-k}$ and taking the differential of $A[k]$ to be the differential of $A$ ( (shifted by $k$ to the "left" or "up").

THEOREM 1.2. - The complex $\operatorname{Tot}\left(\right.$ cube $\left._{n}\left\{A_{\bullet}\right\}\right) \equiv T_{\bullet}$ is naturally quasi isomorphic to $\left(\sigma_{\leqslant n-1} \mathcal{M}\left(A_{\bullet}\right)\right)[1]$. Here $\sigma_{\leqslant n-1}$ denotes the naive truncation of the complex $\mathcal{M}\left(A_{\bullet}\right)$, that is

$$
\left\{\sigma_{\leqslant n-1} \mathcal{M}\left(A_{\bullet}\right)\right\}_{j}= \begin{cases}M_{j} & \text { for } j<n, \\ 0 & \text { for } j \geqslant n,\end{cases}
$$

and the shift $C_{\bullet}[1]$ of a complex $C_{\bullet}$ satisfies $\left(C_{\bullet}[1]\right)_{j}=C_{j-1}$.

Proof. - We first construct a homomorphism of complexes $T_{\bullet} \rightarrow\left(\sigma_{\leqslant n-1} A_{\bullet}\right)[1]$, where we keep the same notation $A_{\bullet}$ for the complex with terms $A_{k}$ and the differential equal to the alternating sum of the face operations. The group $T_{r}$ is a direct sum of $\left(\begin{array}{l}n \\ r\end{array}\right)$ copies of $A_{r-1}$ $(0 \leqslant r \leqslant n)$, which are indexed by $r$-element subsets $S \subset\{0,1, \ldots, n-1\}$. The total differential of $T_{\bullet}$, restricted to the summand $A_{r-1}^{S}$ corresponding to the subset $S=\left\{s_{1}<\cdots<s_{r}\right\}$ is given by the formula

$$
d\left(a^{S}\right)=\sum_{i=1}^{r}(-1)^{i-1} \partial_{i-1}(a)^{\left\{s_{1}, \ldots, \hat{s}_{i}, \ldots, s_{r}\right\}} .
$$

We map $T_{r}$ to $A_{r-1}$, taking the identity map on each copy $A_{r-1}^{S}$ of $A_{r-1}$ in $T_{r}$. The above formula for the differential shows immediately that in this way we get a homomorphism of complexes $T_{\bullet} \rightarrow A_{\bullet}[1]$, which factors through $\left(\sigma_{\leqslant n-1} A_{\bullet}\right)[1]$ since the complex $T_{\bullet}$ is zero in degrees $>n$. We compose this homomorphism with the natural projection

$$
\left(\sigma_{\leqslant n-1} A_{\bullet}\right)[1] \rightarrow\left(\sigma_{\leqslant n-1} \mathcal{M}_{\bullet}\right)[1]
$$

We proceed to show that the resulting map of complexes is a quasi-isomorphism. To do so we recall that each $A_{j}$ is a direct sum of copies of $M_{k}(k \leqslant j)$ indexed by the set $\Phi(j, k)$ of nondecreasing surjective maps $\phi:[j] \rightarrow[k]-$ see [20]

$$
A_{j}=\bigoplus_{k=0}^{j} \bigoplus_{\phi \in \Phi(j, k)} M_{k}
$$


where the copy of $M_{k}$, corresponding to $\phi \in \Phi(j, k)$, is mapped to $A_{j}$ by means of the simplicial operation $\phi^{*}: A_{k} \rightarrow A_{j}$. Each of the face maps respects this direct sum decomposition and does not increase the corresponding index $k$. Thus we may filter the complex $T_{\bullet}$, taking $F_{l}\left(T_{\bullet}\right)$ to be the direct sum of terms $M_{j}$ with $j \leqslant l$. We take a similar filtration on $\mathcal{M}_{\bullet}$ (which happens to be just the canonical filtration). The homomorphism from $T_{\bullet}$ to $\left(\sigma_{\leqslant n-1} \mathcal{M}_{\bullet}\right)[1]$ obviously respects the above filtrations, so to prove our claim it suffices to show that the induced map on quotient complexes $F_{r} / F_{r-1}$ is a quasi-isomorphism for each $0 \leqslant r \leqslant n-1$. In other words we have to verify that if we leave only $M_{r}$ terms in the complex $T_{\bullet}$, then the resulting complex is a resolution of $M_{r}[r+1]$. Denote the complex $F_{r / r-1}\left(T_{\bullet}\right)[-r-1]$ by $D_{\bullet}$. This is a non-negative complex with the following terms:

$$
D_{k}=\bigoplus_{\substack{S \subset\{0,1, \ldots, n-1\} \\|S|=r+k+1}} \bigoplus_{\phi \in \Phi(r+k, r)} M_{r}
$$

The above total sum may be re-indexed using partially defined non-decreasing surjective maps $\phi:[n-1] \rightarrow[r]$ with domain of definition $\operatorname{Dom}(\phi)$, consisting of $r+k+1$ elements. Thus $D_{k}=C_{k}(n, r) \otimes_{\mathbb{Z}} M_{r}$. Moreover one checks easily that the differential of $D_{\bullet}$ coincides with that of $C_{\bullet}(n, r) \otimes_{\mathbb{Z}} M_{r}$, i.e., $D_{\bullet} \cong C_{\bullet}(n, r) \otimes_{\mathbb{Z}} M_{r}$. Thus, it suffices to apply Lemma 1.1.

COROLLARY 1.3. - The homomorphisms in homology induced by the homomorphism of complexes $T_{\bullet} \rightarrow A_{\bullet}[1]$ is an isomorphism in degrees $\leqslant n-1$ and an epimorphism in degree $n$.

Proof. - This follows immediately from the fact that the natural projection $A_{\bullet} \rightarrow \mathcal{M}\left(A_{\bullet}\right)$ is a quasi-isomorphism.

\section{Iterated homotopy cofibres for simplicial spaces}

In this section, we relate the homotopy cofibre of an $n$-cube associated to a simplicial space to the suspension of the Segal realization of the simplicial space. This should be viewed as a "non-abelian" extension of the results of Section 1.

Definition 2.1. - Let $X_{\bullet}$ be a simplicial space. The Segal realization $\left\|X_{\bullet}\right\|$ of $X_{\bullet}$ is the following quotient space of $\coprod_{n=0}^{\infty} X_{n} \times \Delta^{n}$,

$$
\|X .\| \equiv\left(\coprod_{n=0}^{\infty} X_{n} \times \Delta^{n}\right) /\left\langle\left(\delta^{*}(x), t\right) \sim\left(x, \delta_{*}(t)\right)\right\rangle
$$

where $n \geqslant 0, x \in X_{n}, t \in \Delta^{m}$, and $\delta:[m] \rightarrow[n]$ is strictly increasing. The geometric realization of $X_{\bullet}$ is the quotient space of $\left\|X_{\bullet}\right\|$ defined by

$$
\left|X_{\bullet}\right| \equiv\left(\coprod_{n=0}^{\infty} X_{n} \times \Delta^{n}\right) /\left\langle\left(\theta^{*}(x), t\right) \sim\left(x, \theta_{*}(t)\right)\right\rangle
$$

where $n \geqslant 0, x \in X_{n}, t \in \Delta^{m}$, and $\theta:[m] \rightarrow[n]$ is non-decreasing.

Although it is customary to consider the geometric realization $\left|X_{\bullet}\right|$ of a simplicial space $X_{\bullet}$, we shall find it convenient to use the Segal realization on many occasions. The following theorem of Segal tells us that the canonical quotient map is a homotopy equivalence in most cases of interest. 
DEFINITION 2.2.0. - (1) A simplicial space $X$ • is called good, provided that all degeneracy maps $X_{n} \rightarrow X_{n+1}$ are closed cofibrations. For example a level-wise geometric realization of a bisimplicial set is obviously a good simplicial space.

(2) A pointed simplicial space $X_{\bullet}$ is called good if in addition all distinguished points $* \in X_{i}$ are non-degenerate (i.e. $* \hookrightarrow X_{i}$ is a closed cofibration).

Proposition 2.2 (Segal, [26, A.1]).- (1) Let X• be a good simplicial space. Then the canonical quotient map

$$
\left\|X_{\bullet}\right\| \rightarrow\left|X_{\bullet}\right|
$$

is a homotopy equivalence.

(2) Let $X$. be a good pointed simplicial space. Then the canonical quotient map

$$
\left\|X_{\bullet}\right\|_{\text {red }}=\left\|X_{\bullet}\right\| /\|*\| \rightarrow\left|X_{\bullet}\right|
$$

is a homotopy equivalence.

Proof. - The first part of the proposition is proved in [26, A.1]. To prove the second we note that, according to the first part the space $\|*\|$ is contractible. Furthermore one checks easily that the embedding $\|*\| \hookrightarrow\left\|X_{\bullet}\right\|$ is a closed cofibration. These two facts imply that the quotient map $\left\|X_{\bullet}\right\| \rightarrow\left\|X_{\bullet}\right\|_{\text {red }}$ is a homotopy equivalence and hence the quotient map $\left\|X_{\bullet}\right\|_{\text {red }} \rightarrow\left|X_{\bullet}\right|$ is a homotopy equivalence as well.

Let $X_{\bullet}$ be a pointed simplicial space. Then $\left\|X_{\bullet}\right\|$ has a canonical distinguished point $* \in X_{0} \subset\left\|X_{\bullet}\right\|$.

By definition the space $\left\|X_{\bullet}\right\|$ is a quotient of $\coprod_{n \geqslant 0} X_{n} \times \Delta^{n}$. Since the unit interval I is compact, we conclude that the corresponding map

$$
\coprod_{n=0}^{\infty} X_{n} \times \Delta^{n} \times \mathrm{I}=\left(\coprod_{n=0}^{\infty} X_{n} \times \Delta^{n}\right) \times \mathrm{I} \rightarrow\left\|X_{\bullet}\right\| \times \mathrm{I}
$$

is again a quotient map. Since the obvious map $\left\|X_{\bullet}\right\| \times \mathrm{I} \rightarrow \Sigma\left\|X_{\bullet}\right\|$ is also a quotient map, we conclude that $\Sigma\left\|X_{\bullet}\right\|$ is a quotient of $\coprod_{n=0}^{\infty} X_{n} \times \Delta^{n} \times$ I modulo an appropriate equivalence relation. Note further that the canonical map $X_{n} \times \Delta^{n} \times \mathrm{I} \rightarrow \Sigma\left\|X_{\bullet}\right\|$ factors through $X_{n} \times\left(\Delta^{n} \times \mathrm{I} / \Delta^{n} \times 0\right)$. The space $\Delta^{n} \times \mathrm{I} / \Delta^{n} \times 0$ may be identified with $\Delta^{n+1}$ via the map

$$
\Delta^{n} \times \mathrm{I} \stackrel{\left(t_{0}, \ldots, t_{n}\right) \times t \mapsto\left(t t_{0}, \ldots, t t_{n}, 1-t\right)}{\longrightarrow} \Delta^{n+1} .
$$

Furthermore since this quotient map $\Delta^{n} \times \mathrm{I} \rightarrow \Delta^{n+1}$ is proper, we conclude that for any $X$ the corresponding map $X \times \Delta^{n} \times \mathrm{I} \rightarrow X \times \Delta^{n+1}$ is still a quotient map. The above remarks show that for each $n$ the resulting map

$$
X_{n} \times\left(\Delta^{n} \times \mathrm{I} / \Delta^{n} \times 0\right)=X_{n} \times \Delta^{n+1} \rightarrow \Sigma\left\|X_{\bullet}\right\|
$$

is continuous and moreover $\Sigma\left\|X_{\bullet}\right\|$ is a quotient of $\coprod_{n \geqslant 0} X_{n} \times \Delta^{n+1}$ modulo an appropriate equivalence relation.

Since set-theoretically the description of $\Sigma\left\|X_{\bullet}\right\|$ does not present any difficulties. we have proved the following (presumably well known) proposition. 
PROPOSITION 2.3. - For any pointed simplicial space $X_{\bullet}$ the topological space $\Sigma\|X \bullet\|$ is canonically homeomorphic to the quotient space

$$
\coprod_{n=0}^{\infty} X_{n} \times \Delta^{n+1} / \sim
$$

where $\sim$ is an equivalence relation generated by the following identifications

(1) For any strictly increasing map $\theta:[m] \rightarrow[n]$, any $x \in X_{n}$ and any $v \in \Delta^{m+1}$ we have:

$$
\theta^{*}(x) \times v \sim x \times(\tilde{\theta})_{*}(v) .
$$

Here $\tilde{\theta}:[m+1] \rightarrow[n+1]$ is the strictly increasing map taking $m+1$ to $n+1$ and coinciding with $\theta$ on $[\mathrm{m}]$.

(2) For any $x \in X_{n}$ and any $v=\left(v_{0}, \ldots, v_{n}\right) \in \Delta^{n}$

$$
x \times(v, 0) \sim x \times(0, \ldots, 0,1) \sim * .
$$

(3) $* \times v \sim *$ for any $v \in \Delta^{1}$. Here $*$ on the left denotes the distinguished point of $X_{0}$.

Let $Y_{\bullet}, \ldots, \bullet$ be a $n$-cube of pointed spaces. We define the (iterated) cofibre of the $n$-cube $Y_{\bullet}, \ldots, \bullet$ using induction on $n$. A 1 -cube is just a morphism $Y_{1} \stackrel{d_{0}}{\rightarrow} Y_{0}$ of pointed topological spaces and we define the cofiber $\operatorname{cofib}\left(Y_{\bullet}\right)$ as the (reduced) mapping cone of $d_{0}$. In the general case the $n$-cube $Y_{\bullet}, \ldots, \bullet$ defines two $(n-1)$-cubes $Y_{1}=Y_{\bullet}, \ldots, \bullet, 1$ and $Y_{0}=Y_{\bullet}, \ldots, \bullet, 0$ and a morphism $d_{n-1}: Y_{1} \rightarrow Y_{0}$ of $(n-1)$-cubes, so that we may define $\operatorname{cofib}\left(Y_{\bullet}, \ldots, \bullet\right)$ as the (reduced) mapping cone of the corresponding morphism $\operatorname{cofib}\left(d_{n-1}\right): \operatorname{cofib}\left(Y_{1}\right) \rightarrow \operatorname{cofib}\left(Y_{0}\right)$. One checks easily that the iterated cofiber may be also described directly as the quotient space

$$
\operatorname{cofib}\left(Y_{\bullet}, \ldots, \bullet\right) \equiv \bigvee_{S} Y_{S} \wedge \mathrm{I}^{\wedge|S|} / \sim
$$

where $S$ runs through all subsets of $[n-1]$ and the equivalence relation $\sim$ is generated by the identification

$$
y \wedge\left(t_{0} \wedge \cdots \wedge 1_{l} \wedge \cdots \wedge t_{k-1}\right) \sim \partial_{l}(y) \wedge\left(t_{0} \wedge \cdots \wedge \hat{1} \wedge \cdots \wedge t_{k-1}\right) \quad y \in Y_{S}, k=|S| .
$$

The above description of the iterated cofiber is equivalent to the following universal mapping property.

LEMMA 2.4. - Let $Z$ be a pointed topological space. To give a pointed continuous map $p: \operatorname{cofib}\left(Y_{\bullet}, \ldots, \bullet\right) \rightarrow Z$ is the same as to give a family of continuous pointed maps $p_{S}: Y_{S} \wedge \mathrm{I}^{\wedge|S|} \rightarrow Z(S \subset[n-1])$ which satisfy the following compatibility property.

For every $S$ and every $0 \leqslant l \leqslant|S|-1$ the following diagram (in which T denotes the subset of $S$ obtained by deleting the lth element) commutes:

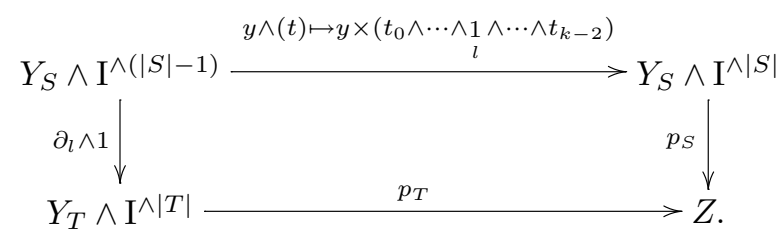


Let $Y_{\bullet}, \ldots, \bullet$ be a $n$-cube of pointed topological spaces and let $Z$ be a compact pointed topological space. In this case $\left(i_{0}, \ldots, i_{n-1}\right) \mapsto Y_{i_{0}, \ldots, i_{n-1}} \wedge Z$ is a new $n$-cube of pointed topological spaces. For each $S \subset[n-1]$ we have a pointed continuous map

$$
\left(Y_{S} \wedge Z\right) \wedge I^{\wedge|S|} \stackrel{\sim}{\rightarrow}\left(Y_{S} \wedge I^{\wedge|S|}\right) \wedge Z \rightarrow \operatorname{cofib}\left(Y_{\bullet}, \ldots, \bullet\right) \wedge Z
$$

These maps are obviously compatible in the sense of Lemma 2.4 and hence define a continuous map $\operatorname{cofib}\left(Y_{\bullet}, \ldots, \bullet \wedge\right) \rightarrow \operatorname{cofib}\left(Y_{\bullet}, \ldots, \bullet\right) \wedge Z$. Induction on $n$ leads easily to the following result.

LEMMA 2.5. - Assume that the space $Z$ is compact. Then for any $n$-cube of pointed spaces $Y$.... we have a natural identification

$$
\operatorname{cofib}\left(Y_{\bullet}, \ldots, \bullet \wedge Z\right)=\operatorname{cofib}\left(Y_{\bullet}, \ldots, \bullet\right) \wedge Z .
$$

In particular, we have a natural identification

$$
\operatorname{cofib}\left(\Sigma^{k} Y_{\bullet}, \ldots, \bullet\right)=\Sigma^{k} \operatorname{cofib}\left(Y_{\bullet}, \ldots, \bullet\right) .
$$

Moreover, let $f_{\bullet} \ldots \bullet: X \bullet \bullet \rightarrow Y_{\bullet} \ldots \bullet$ be a morphism of $n$-cubes of pointed spaces. Let $\mathcal{C}_{i_{0}, \ldots, i_{n-1}}$ denote the cone of the map $f_{i_{0}, \ldots, i_{n-1}}: X_{i_{0}, \ldots, i_{n-1}} \rightarrow Y_{i_{0}, \ldots, i_{n-1}}$. Then the spaces $\mathcal{C} \bullet \ldots$. form $a$ $n$-cube, and we have a natural identification

$$
\operatorname{cofib}\left(\mathcal{C}_{\bullet} \ldots \bullet\right)=\operatorname{cone}\left(\operatorname{cofib}\left(X_{\bullet} \ldots \bullet\right) \rightarrow \operatorname{cofib}\left(Y_{\bullet} \ldots \bullet\right)\right) .
$$

As in Section 1, we associate an $n$-cube of spaces

$$
\operatorname{cube}_{n}\left(X_{\bullet}\right) \equiv Y_{\bullet}, \ldots, \bullet
$$

to a simplicial space $X_{\bullet}$. We now assume that the simplicial space $X_{\bullet}$ is pointed and proceed to construct a natural map

$$
\eta^{X}: \operatorname{cofib}\left(\operatorname{cube}_{n}\left(X_{\bullet}\right)\right) \rightarrow \Sigma\left\|X_{\bullet}\right\| / \Sigma\|*\|=\Sigma\left(\left\|X_{\bullet}\right\|_{\text {red }}\right) .
$$

Since the space $\operatorname{cofib}\left(\operatorname{cube}_{n}\left(X_{\bullet}\right)\right)$ is built out of cubes whereas the suspension $\Sigma\left(\left\|X_{\bullet}\right\|_{\text {red }}\right)$ is built out of simplices, we start the construction of the map

$$
\eta^{X}: \operatorname{cofib}\left(\operatorname{cube}_{n}\left(X_{\bullet}\right)\right) \rightarrow \Sigma\left(\left\|X_{\bullet}\right\|_{\text {red }}\right)
$$

by defining appropriate maps $\eta_{k}: \mathrm{I}^{k} \rightarrow \Delta^{k}(0 \leqslant k \leqslant \infty)$.

LEMMA 2.6. - There exists a sequence of continuous maps $\eta_{k}: \mathrm{I}^{k} \rightarrow \Delta^{k}(0 \leqslant k<\infty)$ with the following properties.

(1) For each $0 \leqslant l \leqslant k-1$ the map $\eta_{k}$ takes the $(k-1)$-dimensional subcube of $\mathrm{I}^{k}$, given by the equation $t_{l}=0$ to the face $t_{k}=0$ of $\Delta^{k}$.

(2) For each $0 \leqslant l \leqslant k-1$ the following diagram commutes

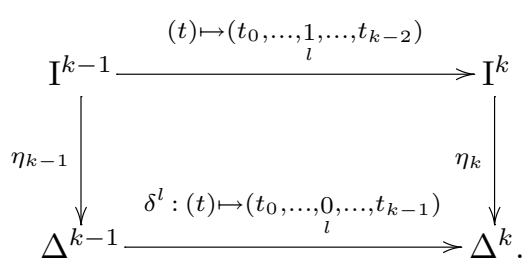


Moreover this sequence of maps is unique up to homotopy. More precisely, if $\eta_{k}$ and $\eta^{\prime}{ }_{k}$ are two families of maps with the above properties then there exists a family of maps $F_{k}: I^{k+1} \rightarrow \Delta^{k}$ such that

(1) $F_{\left.k\right|_{t_{k}=0}}=\eta_{k}, F_{k_{t_{k}=1}}=\eta_{k}^{\prime}$.

(2) For each $0 \leqslant l \leqslant k-1$ the map $F_{k}$ takes the $k$-dimensional subcube of $\mathrm{I}^{k+1}$, given by the equation $t_{l}=0$ to the face $t_{k}=0$ of $\Delta^{k}$.

(3) For each $0 \leqslant l \leqslant k-1$ the following diagram commutes

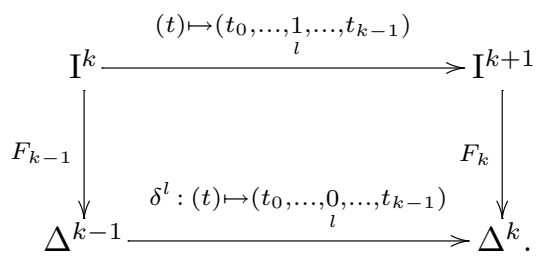

Proof. - Both the existence and the uniqueness are essentially obvious. For example given two families $\eta_{k}$ and $\eta^{\prime}{ }_{k}$ as above one can define the homotopy $F_{k}$ using the formula

$$
F_{k}\left(t_{0}, \ldots, t_{k}\right)=\left(1-t_{k}\right) \eta_{k}\left(t_{0}, \ldots, t_{k-1}\right)+t_{k} \eta^{\prime}{ }_{k}\left(t_{0}, \ldots, t_{k-1}\right) .
$$

To be absolutely precise we fix one family $\eta_{k}$, which we will use in the sequel, by setting

$$
\eta_{k}\left(t_{0}, \ldots, t_{k-1}\right)=\left(1-t_{0}, t_{0}\left(1-t_{1}\right), \ldots, t_{0} \ldots t_{k-2}\left(1-t_{k-1}\right), t_{0} \ldots t_{k-1}\right) .
$$

Remark 2.7. - Let $\eta_{k}$ be the family of maps as above. According to the definition, the map $\eta_{k}$ takes the boundary $\partial \mathrm{I}^{k}$ to the boundary $\partial \Delta^{k}$ and hence defines a map $\bar{\eta}_{k}: \mathrm{I}^{k} / \partial \mathrm{I}^{k} \rightarrow \Delta^{k} / \partial \Delta^{k}$, which is independent (up to homotopy) of the choice of $\eta_{k}$. The spaces $\mathrm{I}^{k} / \partial \mathrm{I}^{k}$ and $\Delta^{k} / \partial \Delta^{k}$ are both homeomorphic to the sphere $S^{k}$. Moreover one checks easily that with our particular choice of $\eta_{k}$ the corresponding map $\bar{\eta}_{k}: \mathrm{I}^{k} / \partial \mathrm{I}^{k} \rightarrow \Delta^{k} / \partial \Delta^{k}$ is a homeomorphism, which we choose to identify these two models of the sphere.

Having fixed a sequence of maps $\eta_{k}$ as above and using Proposition 2.3 and Lemma 2.4, we immediately obtain the desired natural morphisms $\eta^{X}: \operatorname{cofib}\left(\operatorname{cube}_{n}\left(X_{\bullet}\right)\right) \rightarrow \Sigma\left(\left\|X_{\bullet}\right\|_{\text {red }}\right)$.

PROPOSITION 2.8. - For any pointed simplicial space $X_{\bullet}$ and any $n \geqslant 0$ there exists a unique continuous map $\eta^{X}: \operatorname{cofib}\left(\right.$ cube $\left._{n}\left(X_{\bullet}\right)\right) \rightarrow \Sigma\left\|X_{\bullet}\right\| / \Sigma\|*\|$ such that for any subset $S \subset[n-1]$ (containing $k$ elements) the following diagram commutes.

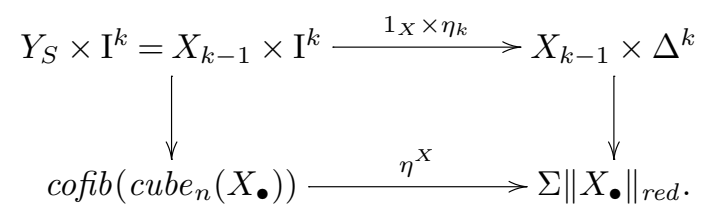

The preceding preliminaries provide the technical basis for the following extension of the results of Section 1 to simplicial spaces.

THEOREM 2.9. - Let $X_{\bullet}$ be a pointed simplicial space. Assume that all distinguished points $* \in X_{i}$ are non-degenerate (i.e. $* \hookrightarrow X_{i}$ is a closed cofibration). Assume further that each $X_{i}$ is $N$-acyclic for some fixed $N \geqslant-1$. In this case the natural map

$$
\eta^{X}: \operatorname{cofib}\left(\operatorname{cube}_{n}\left(X_{\bullet}\right)\right) \rightarrow \Sigma\left\|X_{\bullet}\right\|_{\text {red }}
$$


is an $(N+n+1)$-homology equivalence of $(N+1)$-acyclic spaces, i.e. it induces isomorphisms in singular homology up to degree $N+n$ and an epimorphism in degree $N+n+1$.

Proof. - Denote the $n$-cube $c u b e_{n}\left(X_{\bullet}\right)$ by $Y_{\bullet}, \ldots, \bullet$, the space $c o f i b\left(c u b e_{n}\left(X_{\bullet}\right)\right)$ by $C$, and the space $\Sigma\left\|X_{\bullet}\right\| /$ red by $\Sigma$. Consider the following filtrations of these spaces

$$
\begin{aligned}
& C^{(k)} \equiv \operatorname{Im}\left\{\coprod_{|S| \leqslant k} Y_{S} \times \mathrm{I}^{|S|} \rightarrow C=\operatorname{cofib}\left(Y_{\bullet}, \ldots, \bullet\right)\right\} \quad\left(C^{(k)}=* \text { for } k \leqslant 0\right) . \\
& \Sigma^{(k)} \equiv \operatorname{Im}\left\{\coprod_{j \leqslant k} X_{j-1} \times \Delta^{j} \rightarrow \Sigma=\Sigma\left\|X_{\bullet}\right\|_{\text {red }}\right\} \quad\left(\Sigma^{(k)}=* \text { for } k \leqslant 0\right) .
\end{aligned}
$$

One checks easily that $\Sigma^{(k)}$ (respectively $C^{(k)}$ ) is closed in $\Sigma$ (respectively in $C$ ) and that topology of $\Sigma^{(k)}$ (respectively of $C^{(k)}$ ) is coinduced by the obvious projection

$$
\coprod_{j \leqslant k} X_{j-1} \times \Delta^{j} \rightarrow \Sigma^{(k)}
$$

(respectively $\coprod_{|S| \leqslant k} Y_{S} \times \mathrm{I}^{|S|} \rightarrow C^{(k)}$ ). Using these remarks one concludes further that the space $\Sigma^{(k)}$ is obtained from $\Sigma^{(k-1)}$ by attaching $X_{k-1} \times \Delta^{k}$ along a continuous map

$$
X_{k-1} \times \partial \Delta^{k} \cup * \times \Delta^{k} \rightarrow \Sigma^{(k-1)},
$$

so that we have a cocartesian square

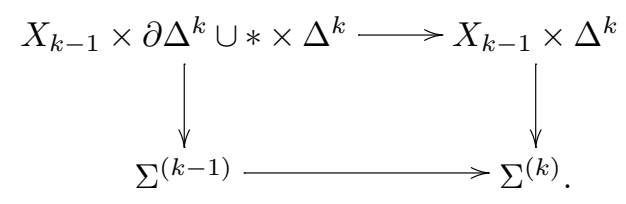

In the same way $C^{(k)}$ is obtained from $C^{(k-1)}$ by attaching $\coprod_{|S|=k} X_{k-1} \times \mathrm{I}^{k}$ along a continuous map $\coprod_{|S|=k}\left(X_{k-1} \times \partial \mathrm{I}^{k} \cup * \times \mathrm{I}^{k}\right) \rightarrow C^{(k-1)}$, so that we have a cocartesian square

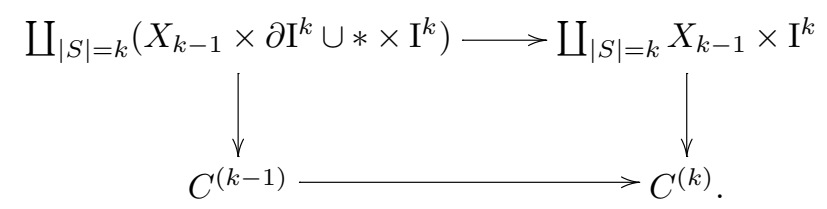

Note that in both cases the top horizontal arrow is a closed cofibration and hence the bottom horizontal arrow is also a closed cofibration. The above filtrations of spaces define induced filtrations on the corresponding singular complexes. In the case of the space $C$ the filtration is obviously finite. In the case of $\Sigma$ one checks easily - see Lemma 2.9.0 below, that every continuous map from a compact space to $\Sigma$ factors through one of $\Sigma^{(k)}$ and hence the singular complex of $\Sigma$ coincides with the direct limit of singular complexes of $\Sigma^{(k)}$. Thus in both cases we get a spectral sequence converging to the reduced homology of the total space and with $E_{1}$-term consisting of relative homology groups. Since $\Sigma^{(k-1)} \hookrightarrow \Sigma^{(k)}$ (and also $C^{(k-1)} \hookrightarrow C^{(k)}$ ) is a closed cofibration we conclude that the $E_{1}$-term of the spectral sequence, corresponding to the filtration of $\Sigma$ looks as follows:

$$
E_{p q}^{1}=H_{p+q}\left(\Sigma^{(p)}, \Sigma^{(p-1)}\right)=\widetilde{H}_{p+q}\left(\Sigma^{(p)} / \Sigma^{(p-1)}\right)=\widetilde{H}_{p+q}\left(X_{p-1} \wedge\left(\Delta^{p} / \partial \Delta^{p}\right)\right)=\widetilde{H}_{q}\left(X_{p-1}\right) .
$$


In the same way we compute the $E^{1}$-term of the spectral sequence corresponding to the filtration of the space $C$

$$
\begin{aligned}
E_{p q}^{\prime 1} & =H_{p+q}\left(C^{(p)}, C^{(p-1)}\right)=\widetilde{H}_{p+q}\left(C^{(p)} / C^{(p-1)}\right)=\widetilde{H}_{p+q}\left(\bigvee_{|S|=p} X_{p-1} \wedge\left(\mathrm{I}^{p} / \partial \mathrm{I}^{p}\right)\right) \\
& =\bigoplus_{|S|=p} \widetilde{H}_{p+q}\left(X_{p-1} \wedge\left(\mathrm{I}^{p} / \partial \mathrm{I}^{p}\right)\right)=\bigoplus_{|S|=p} \widetilde{H}_{q}\left(X_{p-1}\right) .
\end{aligned}
$$

A straightforward computation shows that the differential $d^{1}$ in the spectral sequence $E$

$$
d_{p q}^{1}: E_{p q}^{1}=\widetilde{H}_{q}\left(X_{p-1}\right) \rightarrow \widetilde{H}_{q}\left(X_{p-2}\right)=E_{p, q-1}^{1}
$$

coincides with the alternating sum of maps in homology induced by face operations $\partial_{l}: X_{p-1} \rightarrow X_{p-2}(0 \leqslant l \leqslant p-1)$. In other words the $q$ th row $E_{* q}^{1}$ coincides with the standard complex of the simplicial abelian group $\widetilde{H}_{q}\left(X_{\bullet}\right)$ shifted by one. In the same way the $q$ th row of $E^{\prime 1}$ coincides with the complex $\operatorname{Tot}\left(\operatorname{cube}{ }_{n}\left(\widetilde{H}_{q}\left(X_{\bullet}\right)\right)\right)$. Clearly the map $\eta^{X}$ respects the above filtrations and hence defines a homomorphism of spectral sequences

$$
\begin{aligned}
& \begin{aligned}
{E^{\prime}}_{p q}^{1}=\oplus_{|S|=p} H_{q}\left(X_{p-1}\right) & \Longrightarrow H_{p+q}(C) \\
& \downarrow\left(\eta^{X}\right)_{*}
\end{aligned} \\
& E_{p q}^{1}=H_{q}\left(X_{p-1}\right) \quad \Longrightarrow \quad H_{p+q}(\Sigma) .
\end{aligned}
$$

Remark 2.7 shows that the homomorphism of complexes $E_{* q}^{\prime 1} \rightarrow E_{* q}^{1}$ is nothing but the map considered in Section 1 applied to the simplicial abelian group $\widetilde{H}_{q}\left(X_{\bullet}\right)$. Corollary 1.3 implies now that the homomorphism $E_{p, q}^{\prime 2} \stackrel{\left(\eta^{X}\right)_{*}}{\longrightarrow} E_{p, q}^{2}$ is an isomorphism for $p<n$ and an epimorphism for $p=n$ (with $q$ arbitrary). Since $E_{p, q}^{2}=E_{p, q}^{\prime 2}=0$ for $q \leqslant N$ and $p$ arbitrary, we conclude that the map $E_{p, q}^{\prime 2} \stackrel{\left(\eta^{X}\right)_{*}}{\longrightarrow} E_{p, q}^{2}$ is an isomorphism for $p+q \leqslant n+N$ and an epimorphism for $p+q=n+N+1$. The standard comparison theorem for spectral sequences implies immediately that the map on abutments

$$
\left(\eta^{X}\right)_{*}: H_{i}\left(\operatorname{cofib}\left\{\operatorname{cube}_{n}\left(X_{\bullet}\right)\right\}\right) \rightarrow H_{i}\left(\Sigma\left\|X_{\bullet}\right\|_{r e d}\right)
$$

is an isomorphism in degrees $\leqslant n+N$ and an epimorphism in degree $n+N+1$.

LEMMA 2.9.0. - In conditions and notations of the previous proof every continuous map $f: Z \rightarrow \Sigma$ from a compact space $Z$ to $\Sigma$ factors through one of $\Sigma^{(k)}$.

Proof. - Set $Z^{(k)}=f^{-1}\left(\Sigma^{(k)}\right)$ and assume that $Z^{(k)} \neq Z$ for all $k$. Choose a point $z_{k} \in$ $Z \backslash Z^{(k)}$. Since the space $Z$ is compact the sequence $\left\{z_{k}\right\}_{k=1}^{\infty}$ contains a converging subsequence. To simplify notations we assume that the sequence $\left\{z_{k}\right\}_{k=1}^{\infty}$ converges itself and denote by $z \in Z$ its limit: $z=\lim _{k \rightarrow \infty} z_{k}$. Fix an integer $n$ such that $f(z) \in \Sigma^{(n)}$. Since $f$ is continuous we conclude that $f(z)=\lim _{k \rightarrow \infty} f\left(z_{k}\right)$. Now we are going to construct an open neighborhood $f(z) \in U \subset \Sigma$, such that $f\left(z_{k}\right) \notin U$ for $k \geqslant n$ which would obviously give a contradiction and thus complete the proof. To do so we construct using induction on $N \geqslant n$ an open neighborhood $U_{N}$ of $f(z)$ in $\Sigma^{(N)}$ such that $f\left(z_{k}\right) \notin U_{N}$ for $k \geqslant n$ and $U_{N} \cap \Sigma^{(N-1)}=U_{N-1}$ for $N>n$. We start with any open neighborhood $U_{n}$ of $f(z)$ in $\Sigma^{(n)}$ and note that according to our choice $f\left(z_{k}\right) \notin \Sigma^{(n)}$ for $k \geqslant n$ and, in particular, $f\left(z_{k}\right) \notin U_{n}$ for $k \geqslant n$. Assume now that $U_{N-1}$ with the desired property is already constructed. Start with an open subset $V \subset \Sigma^{(N)}$ such that $V \cap \Sigma^{(N-1)}=U_{N-1}$. Note next that the only members of the sequence $f\left(z_{k}\right)(k \geqslant n)$ which could be in $V$ are $f\left(z_{n}\right), \ldots, f\left(z_{N-1}\right)$ and moreover these possibly bad members lie in 


$$
V \backslash U_{N-1} \subset \Sigma^{(N)} \backslash \Sigma^{(N-1)}=\left(X_{N-1} \backslash *\right) \times\left(\Delta^{N} \backslash \partial \Delta^{N}\right) .
$$

Note finally that every closed cofibration is a zero set - see [23], thus there exists a continuous function $u: X_{N-1} \rightarrow \mathrm{I}$ such that $u^{-1}(0)=*$. This readily implies that there exist closed subsets $A \subset X_{N-1}, B \subset \Delta^{N}$ such that $* \notin A, B \cap \partial \Delta^{N}=\emptyset$ and $\left\{f\left(z_{n}\right), \ldots, f\left(z_{N-1}\right)\right\} \cap V \subset A \times B$ Now it suffices to set $U_{N}=V \backslash A \times B$.

We keep the same notation $\eta^{X}$ for the composition map

$$
\operatorname{cofib}\left(\operatorname{cube}_{n}\left(X_{\bullet}\right)\right) \rightarrow \Sigma\left\|X_{\bullet}\right\|_{r e d} \rightarrow \Sigma\left|X_{\bullet}\right|
$$

Combining the Theorem 2.9 with the Theorem of Segal (Proposition 2.2 above) we get easily the following corollary.

Corollary 2.9.1. - Let $X_{\bullet}$ be a good pointed simplicial space. Assume that each $X_{i}$ is $N$-acyclic for some fixed $N \geqslant-1$. In this case the natural map

$$
\eta^{X}: \operatorname{cofib}\left(\operatorname{cube}_{n}\left(X_{\bullet}\right)\right) \rightarrow \Sigma\left|X_{\bullet}\right|
$$

is an $(N+n+1)$-homology equivalence of $(N+1)$-acyclic spaces.

We recall that a continuous map $f: X \rightarrow Y$ between connected pointed spaces is said to be an $n$-equivalence (or, equivalently, $n$-connected) provided that the induced map on homotopy groups $\pi_{i}(X) \rightarrow \pi_{i}(Y)$ is injective for $i<n$ and surjective for $i \leqslant n$. In particular, a pointed space $X$ is said to be $n$-connected provided that the map from the base point to $X$ is $n$-connected (i.e., provided that $\pi_{i}(X)=0$ for $\left.i \leqslant n\right)$.

COROLlaRY 2.10. - Let $X_{\bullet}$ be a good pointed simplicial space. Assume further that $N \geqslant 0$ and each $X_{i}$ is $N$-connected, then the natural map

$$
\eta^{X}: \operatorname{cofib}\left(\operatorname{cube}_{n}\left(X_{\bullet}\right)\right) \rightarrow \Sigma\left|X_{\bullet}\right|
$$

is an $(N+n+1)$-equivalence of $(N+1)$-connected spaces.

Proof. - One checks easily (using the van Kampen Theorem) that under our assumptions both domain and range of $\eta^{X}$ are simply connected so that our statement follows from Theorem 2.9 and the Whitehead Theorem.

We finish this section by observing in Corollary 2.12 that construction of the canonical map $\eta^{X}: \operatorname{cofib}\left(\operatorname{cube}_{n}\left(X_{\bullet}\right)\right) \rightarrow \Sigma\left(\left|X_{\bullet}\right|\right)$ admits a natural extension from simplicial spaces to simplicial prespectra. Such an extension requires the following observation which follows from the associativity of the smash product.

Lemma 2.11. - Let $X \bullet$ be any simplicial space. Then the following diagram of pointed spaces commutes

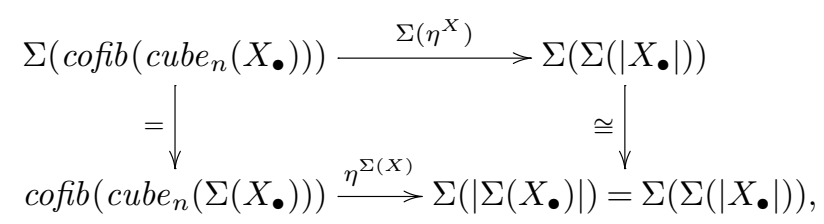

where the right vertical arrow interchanges the two suspensions. 
COROLlary 2.12. - Let $X_{\bullet}$ be a simplicial prespectrum. The family of maps

$$
\eta^{X^{i}}: \operatorname{cofib}\left(\operatorname{cube}_{n}\left(X_{\bullet}^{i}\right)\right) \rightarrow \Sigma\left(\left|X_{\bullet}^{i}\right|\right)
$$

is a morphism of prespectra. Moreover the following diagram of homotopy groups (in which the vertical arrows are suspension homomorphisms) commutes up to a sign

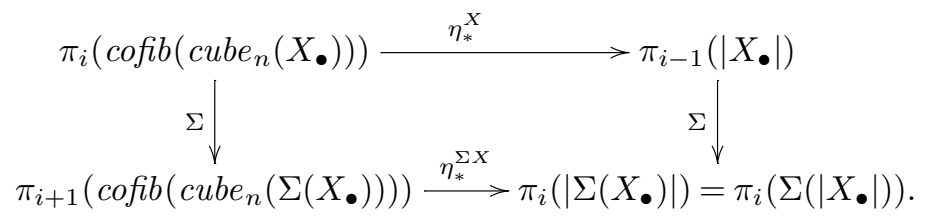

\section{Comparison of iterated homotopy fibres and cofibres}

As we saw in Corollary 2.10, the iterated cofibre $\operatorname{cofib}\left(\operatorname{cube}_{n}\left(X_{\bullet}\right)\right)$ of the $n$-cube associated to a simplicial space $X_{\bullet}$ is closely related to the suspension of the geometric realization $\Sigma\left|X_{\bullet}\right|$. On the other hand, techniques developed for the study of algebraic $K$-theory have utilized the iterated homotopy fibre; namely, multi-relative $K$-theory can be interpreted as an iterated homotopy fibre of an $n$-cube of spaces obtained by applying the $K$-functor to an $n$-cube of schemes. The purpose of this section is to prove in Proposition 3.4 a comparison between iterated fibres and iterated cofibres of $n$-cubes of spaces. Although the material we present here may well be known to experts, we work through the proofs for want of a suitable reference.

We proceed by induction on $n$ to define $f i b\left(Y_{\bullet}, \ldots, \bullet\right)$, the homotopy fibre of the $n$-cube $Y_{\bullet}, \ldots, \bullet$ of pointed spaces. For $n=1$, we define $f i b\left(Y_{\bullet}\right)$ as the homotopy fiber $f i b\left\{Y_{1} \stackrel{d_{0}}{\rightarrow} Y_{0}\right\}$ of the structure map $d_{0}: Y_{1} \rightarrow Y_{0}$. Having defined the iterated fibre for $(n-1)$-cubes of pointed spaces, we proceed as in Section 2 to define $f i b\left(Y_{\bullet}, \ldots, \bullet\right)$ for the $n$-cube $Y_{\bullet}, \ldots, \bullet$. Namely, we consider two $(n-1)$-cubes $Y_{1}=Y_{\bullet}, \ldots, \bullet, 1$ and $Y_{0}=Y_{\bullet}, \ldots, \bullet, 0$ and the structure map $d_{n-1}: Y_{1} \rightarrow Y_{0}$ and we define $f i b\left(Y_{\bullet}, \ldots, \bullet\right)$ as the homotopy fiber of the induced map $f i b\left(d_{n-1}\right): f i b\left(Y_{1}\right) \rightarrow f i b\left(Y_{0}\right)$.

One can easily provide an explicit description of $f i b\left(Y_{\bullet}, \ldots, \bullet\right)$ similar to the description of the iterated cofiber given in Section 2, which presents $f i b\left(Y_{\bullet} . . \bullet\right)$ as a subspace in the product space of function spaces

$$
f i b\left(Y_{\bullet} \ldots\right) \subset \prod_{S \subset[n-1]}\left(Y_{S}\right)^{\left(I^{n-|S|}\right)} .
$$

Namely, for any $S \subset[n-1]$ denote by $I_{S}^{n} \cong I^{n-|S|}$ the subcube of $I^{n}$ given by equations $t_{i}=0$ $(i \in S)$. The subspace $f i b\left(Y_{\bullet} \bullet \bullet\right) \subset \prod_{S \subset[n-1]}\left(Y_{S}\right)^{\left(I^{n-|S|}\right)}$ consists of families of continuous maps $\omega=\left\{\omega_{S}: I_{S}^{n} \rightarrow Y_{S}\right\}_{S \subset[n-1]}$ which satisfy the following compatibility properties

(1) $\omega_{S}(t)=*$ if at least one of the coordinates of $t$ equals 1 .

(2) Let $T$ be obtained from $S$ by deleting the $l$ th element $(0 \leqslant l \leqslant|S|-1)$. Then the following diagram commutes

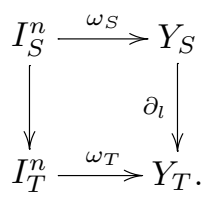


Proceeding in the same way as in Section 2, one proves easily the following result dual to Lemma 2.5.

LEMMA 3.1. - For any $n$-cube of pointed spaces $Y_{\bullet} \ldots \bullet$ we have a natural identification

$$
f i b\left(\Omega^{k} Y_{\bullet}, \ldots, \bullet\right)=\Omega^{k} f i b\left(Y_{\bullet}, \ldots, \bullet\right) .
$$

Moreover, let $f_{\bullet} \ldots \bullet: X \bullet \ldots \bullet \rightarrow Y_{\bullet} \ldots \bullet$ be a morphism of $n$-cubes and let $\mathcal{F}_{i_{0}, \ldots, i_{n-1}}$ denote the homotopy fiber of the map

$$
f_{i_{0}, \ldots, i_{n-1}}: X_{i_{0}, \ldots, i_{n-1}} \rightarrow Y_{i_{0}, \ldots, i_{n-1}} .
$$

Then the spaces $\mathcal{F}_{\bullet . . .}$ form a $n$-cube and moreover we have a natural identification

$$
f i b\left(\mathcal{F}_{\bullet} \ldots \bullet\right)=f i b\left(f i b\left(X_{\bullet} \ldots \bullet\right) \rightarrow f i b\left(Y_{\bullet} \ldots \bullet\right)\right) .
$$

We next proceed to define a map $f i b\left(Y_{\bullet} \ldots \bullet\right) \rightarrow \Omega^{n}\left(\operatorname{cofib}\left(Y_{\bullet} \ldots \bullet\right)\right)$. We start with the case $n=1$, in which case we have to define a map from the homotopy fiber to loops on the homotopy cofiber for an arbitrary continuous map of pointed spaces $f: X \rightarrow Y$.

We define a map

$$
\Omega_{f}: Q(f) \rightarrow \mathcal{P}(\operatorname{cofib}(f))
$$

by sending $(x \in X, \tau: \mathrm{I} \rightarrow Y)$ to the path $\omega$ defined by sending $t \in I$ to the image of $x \wedge 2 t$ in $\operatorname{cofib}(f)$ for $0 \leqslant t \leqslant 1 / 2$ and to $\tau(2 t-1)$ for $1 / 2 \leqslant t \leqslant 1$. We readily verify that $\Omega_{f}$ is continuous and determines a commutative diagram

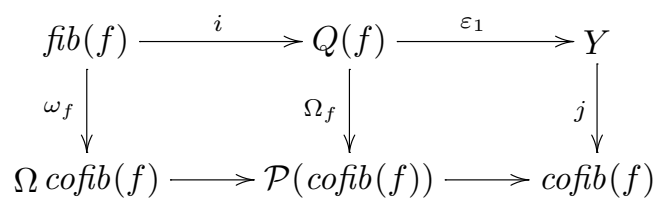

where $i: f i b(f) \rightarrow Q(f), j: Y \rightarrow c o f i b(f)$ are the structure embeddings.

In the general case we use induction on $n$ and define the map $f i b\left(Y_{\bullet} \ldots \bullet\right) \rightarrow \Omega^{n}\left(\operatorname{cofib}\left(Y_{\bullet} \ldots \bullet\right)\right)$ as the composition

$$
\begin{aligned}
f i b\left(Y_{\bullet} . \bullet\right. & =f i b\left\{f i b\left(Y_{1}\right) \rightarrow f i b\left(Y_{0}\right)\right\} \rightarrow f i b\left\{\Omega^{n-1}\left(\operatorname{cofib}\left(Y_{1}\right)\right) \rightarrow \Omega^{n-1}\left(\operatorname{cofib}\left(Y_{0}\right)\right)\right\} \\
& =\Omega^{n-1}\left(f i b\left\{\operatorname{cofib}\left(Y_{1}\right) \rightarrow \operatorname{cofib}\left(Y_{0}\right)\right\}\right) \rightarrow \Omega^{n-1}\left(\Omega\left(\operatorname{cofib}\left\{\operatorname{cofib}\left(Y_{1}\right) \rightarrow \operatorname{cofib}\left(Y_{0}\right)\right\}\right)\right) \\
& =\Omega^{n}\left(\operatorname{cofib}\left(Y_{\bullet} \ldots \bullet\right)\right) .
\end{aligned}
$$

We denote the resulting map $f i b\left(Y_{\bullet} \ldots \bullet\right) \rightarrow \Omega^{n}\left(\operatorname{cofib}\left(Y_{\bullet} \ldots \bullet\right)\right)$ by $\rho$ or $\rho^{Y}$. We use the notation $\rho_{Y}: \Sigma^{n}\left(f i b\left(Y_{\bullet} \ldots \bullet\right)\right) \rightarrow \operatorname{cofib}\left(Y_{\bullet} \ldots \bullet\right)$ for the adjoint of $\rho^{Y}$.

The preceding explicit description of $\omega_{f}$ in the case $n=1$ easily yields the following explicit formula

$$
\rho^{Y}(\omega)(t)=\omega_{S}(\overline{2 t-1}) \wedge\left(2 t_{i_{0}} \wedge \cdots \wedge 2 t_{i_{k-1}}\right) .
$$

Here $S=\left\{i_{0}<\cdots<i_{k-1}\right\}=\left\{i \in[n-1]: t_{i} \leqslant 1 / 2\right\}$, and the point $\overline{2 t-1}$ is obtained from $2 t-1$ replacing all negative coordinates by 0 .

Using (for example) this formula one establishes immediately the following fact. 
Lemma 3.2. - For any $(n+1)$-cube $Y=Y_{\bullet} \ldots$ of pointed spaces the following diagram commutes up to (base point preserving) homotopy

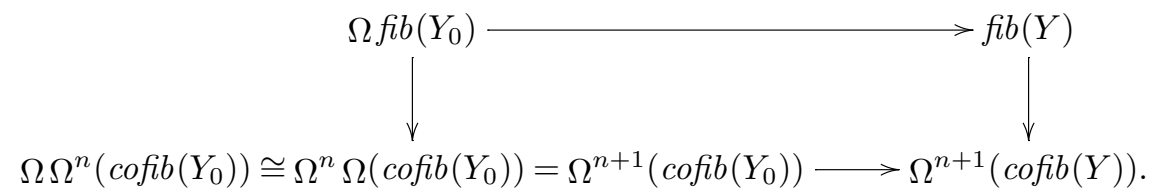

Lemma 3.2 immediately gives us the following useful fact relating the boundary map $\delta$ in the long exact sequence for the fibration sequence $f i b(Y) \rightarrow f i b\left(Y_{1}\right) \stackrel{d_{n}}{\rightarrow} f i b\left(Y_{0}\right)$ to the map on homotopy groups of iterated cofibres induced by the lower horizontal map of the above square.

COROLlary 3.2.1. - The following diagram of homotopy groups commutes up to a sign $(-1)^{n}$.

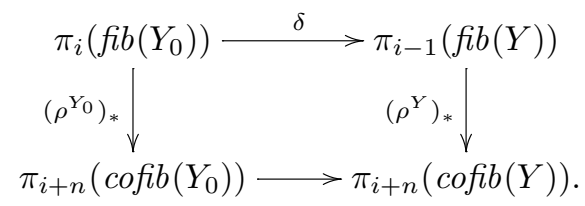

We will also need the following result, which is an easy application of Corollary 3.2.1.

COROLlARY 3.2.2. - Let $f: X \rightarrow Y$ be a morphism of n-cubes. Let $\mathcal{F}=\mathcal{F}_{\bullet} \ldots \bullet$ (respectively $\mathcal{C}=\mathcal{C}_{\bullet} \ldots \bullet$ ) denote the $n$-cube of homotopy fibers (respectively cofibers) of $f$. The following diagram of homotopy groups commutes up to a sign

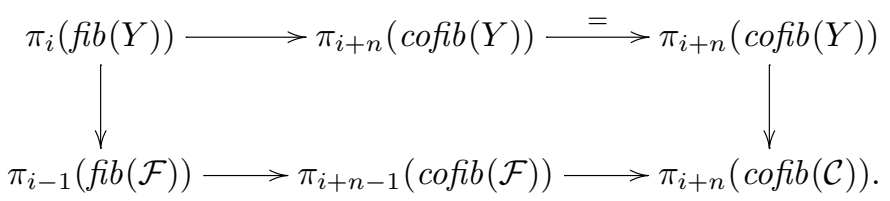

Here the last arrow at the bottom line is the composition of the suspension homomorphism $\pi_{i+n-1}(\operatorname{cofib}(\mathcal{F})) \rightarrow \pi_{i+n}(\Sigma \operatorname{cofib}(\mathcal{F}))=\pi_{i+n}(\operatorname{cofib}(\Sigma \mathcal{F}))$ and the homomorphism in homotopy groups induced by the morphism of $n$-cubes $\Sigma \mathcal{F} \rightarrow \mathcal{C}$.

Proof. - Let $Z$ denote the $(n+1)$-cube such that $Z_{0}=X, Z_{1}=Y$ and the structure morphism $d_{n}$ coincides with $f$. Lemmas 3.1 and 2.5 provide us with canonical identifications

$$
f i b(\mathcal{F})=f i b(Z), \quad \operatorname{cofib}(\mathcal{C})=\operatorname{cofib}(Z) .
$$

Now it is easy to see that the composition of the bottom row of our diagram coincides with the map induced by $\rho^{Z}: f i b(\mathcal{F})=f i b(Z) \rightarrow \Omega^{n+1}(\operatorname{cofib}(Z))=\Omega^{n+1}(\operatorname{cofib}(\mathcal{C}))$.

Our main objective in this section is to show that the resulting map

$$
f i b\left(Y_{\bullet} \ldots \bullet\right) \rightarrow \Omega^{n}\left(\operatorname{cofib}\left(Y_{\bullet} \ldots \bullet\right)\right)
$$

is an equivalence up to a certain degree. As always we start with the case $n=1$.

$4^{\mathrm{e}}$ SÉRIE - TOME $35-2002-\mathrm{N}^{\circ} 6$ 
Proposition 3.3. - Let $f: X \rightarrow Y$ be a map of $N$-connected, pointed spaces. Assume that distinguished points in $X$ and $Y$ are non-degenerate. Then the space cofib $(f)$ is also $N$-connected and the map of (3.2.0)

$$
\omega_{f}: f i b(f) \rightarrow \Omega \operatorname{cofib}(f)
$$

is a $2 N$-equivalence (i.e., the induced map on $\pi_{i}$ is an isomorphism in degrees $<2 N$ and an epimorphism in degree $2 N)$ of $(N-1)$-connected spaces.

Proof. - In cases $N=-1,0$ there is nothing to prove, so assume that $N \geqslant 1$. The Theorem of van Kampen readily implies that in this case the space $\operatorname{cofib}(f)$ is simply connected. Since its homology groups are related to homology groups of $X$ and $Y$ by a long exact sequence we immediately conclude that it is $N$-acyclic and hence also $N$-connected in view of the Hurewitz Theorem. The space $f i b(f)$ is obviously $(N-1)$-connected so it suffices to show that $\omega_{f}$ is a $2 N$-equivalence; in other words, we must show that the induced map in homotopy groups

$$
\pi_{i}(f i b(f)) \stackrel{\left(\omega_{f}\right)_{*}}{\longrightarrow} \pi_{i}(\Omega(\operatorname{cofib}(f)))=\pi_{i+1}(\operatorname{cofib}(f))
$$

is an isomorphism in degrees $<2 N$ and an epimorphism in degree $2 N$. To do so we set $\mathcal{F}=f i b(f), \mathcal{C}=\operatorname{cofib}(f)$ and denote further by $Z$ the reduced mapping cylinder of $f$, so that $\mathcal{C}=\operatorname{cofib}(f)$ is obtained from $Z$ by collapsing the subspace $X \times 0$ to a point. Since all the spaces in question are simply connected, the pair $(Z, X \times 0)$ is $N$-connected and the space $X \times 0$ is $N$-connected as well, the Blakers-Massey Homotopy Excision Theorem (cf. [40]) shows that the canonical map

$$
p_{*}: \pi_{s}(Z, X \times 0) \rightarrow \pi_{s}(\mathcal{C})
$$

is an isomorphism in degrees $s \leqslant 2 N$ and an epimorphism in degree $2 N+1$. Moreover the above exhibited map $\omega_{f}: \mathcal{F} \rightarrow \Omega \mathcal{C}$ corresponds to a map $\mathcal{F} \times I \rightarrow \mathcal{C}$, which has an obvious lifting to a map $\omega^{f}: \mathcal{F} \times I \rightarrow Z$ such that $\omega^{f}((x, \tau), 0)=(x, 0)$. The map $\omega^{f}$ defines canonical homomorphisms $\left(\omega^{f}\right)_{*}: \pi_{i}(\mathcal{F}) \rightarrow \pi_{i+1}(Z, X \times 0)$ and the composition $p_{*} \circ\left(\omega^{f}\right)_{*}$ coincides with $\left(\omega_{f}\right)_{*}$. Finally the homomorphisms $\left(\omega^{f}\right)_{*}: \pi_{i}(\mathcal{F}) \rightarrow \pi_{i+1}(Z, X \times 0)$ are isomorphisms in all degrees as one sees comparing the long exact sequence of the fibration $\mathcal{F} \rightarrow X \rightarrow Y$ to the long exact sequence of the pair $(Z, X \times 0)$.

We now extend Proposition 3.3 in the evident way to $n$-cubes of pointed spaces.

Proposition 3.4. - Let $Y_{\bullet} \ldots$ • be a n-cube of $N$-connected spaces with non-degenerate distinguished points. Then the space cofib $\left(Y_{\bullet} \ldots \bullet\right)$ is also $N$-connected and the natural map

$$
f i b\left(Y_{\bullet} \ldots \bullet\right) \rightarrow \Omega^{n}\left(\operatorname{cofib}\left(Y_{\bullet} \ldots \bullet\right)\right)
$$

is a $(2 N-n+1)$-equivalence of $(N-n)$-connected spaces.

Proof. - We proceed by induction on $n$. The case $n=1$ was settled above. Assume that $n>1$ and denote by $Y_{1}=Y_{\bullet}, \ldots, \bullet, 1, Y_{0}=Y_{\bullet}, \ldots, \bullet, 0$ the corresponding $(n-1)$-cubes. The maps $f i b\left(Y_{1}\right) \rightarrow \Omega^{n-1}\left(\operatorname{cofib}\left(Y_{1}\right)\right), f i b\left(Y_{0}\right) \rightarrow \Omega^{n-1}\left(\operatorname{cofib}\left(Y_{0}\right)\right)$ are $(2 N-n+2)$-equivalences according to the induction hypothesis. This implies that the induced map on the homotopy fibers

$$
\begin{aligned}
f i b\left(Y_{\bullet} . . \bullet\right) & =f i b\left(f i b\left(Y_{1}\right) \rightarrow f i b\left(Y_{0}\right)\right) \rightarrow f i b\left(\Omega^{n-1}\left(\operatorname{cofib}\left(Y_{1}\right)\right) \rightarrow \Omega^{n-1}\left(\operatorname{cofib}\left(Y_{0}\right)\right)\right) \\
& =\Omega^{n-1}\left(f i b\left(\operatorname{cofib}\left(Y_{1}\right) \rightarrow \operatorname{cofib}\left(Y_{0}\right)\right)\right)
\end{aligned}
$$


is a $(2 N-n+1)$-equivalence. Furthermore the spaces $\operatorname{cofib}\left(Y_{1}\right), \operatorname{cofib}\left(Y_{0}\right)$ are still $N$-connected according to the induction hypothesis and hence the map

$$
f i b\left(\operatorname{cofib}\left(Y_{1}\right) \rightarrow \operatorname{cofib}\left(Y_{0}\right)\right) \rightarrow \Omega \operatorname{cofib}\left(\operatorname{cofib}\left(Y_{1}\right) \rightarrow \operatorname{cofib}\left(Y_{0}\right)\right)=\Omega \operatorname{cofib}\left(Y_{\bullet} \ldots \bullet\right)
$$

is a $2 N$-equivalence according to Proposition 3.3. Thus the induced map on loop spaces

$$
\Omega^{n-1}\left(f i b\left(\operatorname{cofib}\left(Y_{1}\right) \rightarrow \operatorname{cofib}\left(Y_{0}\right)\right)\right) \rightarrow \Omega^{n} \operatorname{cofib}\left(Y_{\bullet} \ldots \bullet\right)
$$

is a $(2 N-(n-1))$-equivalence.

\section{Iterated fibers for simplicial prespectra}

In this section, we extend the results of the previous sections from simplicial spaces to simplicial prespectra (i.e., simplicial objects in the category of prespectra as discussed in Appendix B). The need to pass to spectra (or their more rigid formulation as prespectra) can be seen in the formulation of Proposition 3.4. In applications, the connectivity (designated $N$ in Proposition 3.4) of pointed spaces constituting an $n$-cube will be fixed (typically, $N=-1$ ) but we shall wish to consider arbitrarily large $n$-cubes. Another advantage of this extension is that it permits us to consider negative homotopy groups (in applications, we shall consider $\pi_{-1}$ ). In Theorem 4.3, we present the extension to simplicial prespectra of the investigation of the map on homotopy groups

$$
\pi_{i}\left(f i b\left(\operatorname{cube}_{n}\left(X_{\bullet}\right)\right)\right) \rightarrow \pi_{i+n-1}\left(\left|X_{\bullet}\right|\right)
$$

provided by Corollary 2.10 and Proposition 3.4.

We refer the reader to Appendix B for our conventions concerning prespectra. In particular, distinguished points in all spaces constituting a prespectrum are always assumed non-degenerate, and a prespectrum $X=\left(X^{0}, X^{1}, \ldots\right)$ is said to be $N$-connected if $X^{k}$ is $(N+k)$-connected for each $k \geqslant 0$. We recall that the $n$th homotopy group of the prespectrum $X$ is defined as

$$
\pi_{n}(X)=\lim _{k \geqslant-n} \pi_{n+k}\left(X^{k}\right) \quad(-\infty<n<\infty)
$$

A map of prespectra $f: X \rightarrow Y$ is said to be a weak equivalence provided that $f$ induces isomorphisms $f_{*}: \pi_{j}(X) \stackrel{\sim}{\rightarrow} \pi_{j}(Y)$ for all $j$.

Let $Y_{\bullet} \ldots$ be a $n$-cube in the category of prespectra. We denote the spaces constituting the prespectrum $Y_{i_{0}, \ldots, i_{n-1}}$ by $Y_{i_{0}, \ldots, i_{n-1}}^{k}(k=0,1, \ldots)$. For each $k$ the spaces $Y_{i_{0}, \ldots, i_{n-1}}^{k}$ give us a $n$-cube in the category of pointed spaces and hence we may consider the spaces $\operatorname{cofib}\left(Y_{\bullet}^{k} \bullet_{\bullet}\right)$ and $f i b\left(Y_{\bullet}^{k} \bullet \bullet\right)$. Moreover the structure maps $\Sigma\left(Y_{\bullet}^{k} \bullet \bullet\right) \rightarrow Y_{\bullet}^{k+1}$ give us a morphism of $n$-cubes and hence we get the induced map on cofibers

$$
\Sigma\left(\operatorname{cofib}\left(Y_{\bullet \ldots \bullet}^{k}\right)\right)=\operatorname{cofib}\left(\Sigma\left(Y_{\bullet \ldots \bullet}^{k}\right)\right) \rightarrow \operatorname{cofib}\left(Y_{\bullet \ldots \bullet}^{k+1}\right)
$$

In this way we get a prespectrum

$$
\operatorname{cofib}\left(Y_{\bullet} \ldots \bullet\right)=\left(\operatorname{cofib}\left(Y_{\bullet}^{0} \ldots \bullet\right), \operatorname{cofib}\left(Y_{\bullet}^{1} \ldots \bullet\right), \ldots\right) .
$$

In the same way we verify that the spaces $f i b\left(Y_{\bullet}^{k} \bullet\right.$ ) constitute a prespectrum which we denote $f i b\left(Y_{\bullet} ..\right)$. The following lemma sums up some of the elementary properties of these operations. 
Lemma 4.1. - (a) Assume that $Y_{\bullet} \ldots$ is an $n$-cube in the category of $\Omega$-prespectra. Then $f i b\left(Y_{\bullet} \ldots \bullet\right)$ is also a $\Omega$-prespectrum.

(b) Assume that the prespectra $Y_{i_{0}, \ldots, i_{n-1}}$ are $N$-connected. Then the prespectrum fib $\left(Y_{\bullet} \ldots \bullet\right)$ is $(N-n)$-connected and the prespectrum cofib $\left(Y_{\bullet} \ldots \bullet\right)$ is $N$-connected.

(c) The family of maps $f i b\left(Y_{\bullet}^{k} \bullet\right) \rightarrow \Omega^{n} \operatorname{cofib}\left(Y_{\bullet}^{k} . \bullet\right)$ is a morphism of prespectra.

Proof. - To prove (a), it suffices to establish that the homotopy fiber of a morphism of $\Omega$-prespectra is again a $\Omega$-prespectrum, which is straightforward from definitions. Assertion (b) follows immediately from Proposition 3.4. To prove (c), it suffices (in view of the inductive definition of the map in question) to consider the case $n=1$, in which case our statement is straightforward.

The following prespectrum analogue of Proposition 3.4 has the strong conclusion of a weak equivalence rather than an equivalence in a range bounded by the connectivity.

PROPOSITION 4.2. - Let $Y_{\bullet} \ldots$ • be an $n$-cube in the category of $N$-connected prespectra. Then $f i b\left(Y_{\bullet} . . \bullet\right)$ is an $(N-n)$-connected prespectrum, cofib $\left(Y_{\bullet} \ldots \bullet\right)$ is an $N$-connected prespectrum and the natural morphisms

$$
\begin{aligned}
& f i b(Y \bullet \ldots \bullet) \stackrel{\rho^{Y}}{\longrightarrow} \Omega^{n}\left(\operatorname{cofib}\left(Y_{\bullet} \ldots \bullet\right)\right) \\
& \Sigma^{n}(f i b(Y \bullet \ldots \bullet)) \stackrel{\rho_{Y}}{\longrightarrow} \operatorname{cofib}\left(Y_{\bullet} \ldots \bullet\right)
\end{aligned}
$$

are weak equivalences of prespectra.

Proof. - For each $k$, we may apply Proposition 3.4 to the maps of pointed spaces

$$
f i b\left(Y_{\bullet}^{k} \bullet \bullet\right) \stackrel{\rho^{Y^{k}}}{\longrightarrow} \Omega^{n}\left(\operatorname{cofib}\left(Y_{\bullet}^{k} \bullet \bullet\right)\right)
$$

associated to the $n$-cube $Y_{\bullet}^{k} \ldots$. of $N+k$-connected spaces to conclude that $\rho^{Y^{k}}$ induces isomorphisms in homotopy groups up to degree $2 N+2 k-n$. Passing to the limit on $k$ we see immediately that the map in homotopy groups induced by the morphism of prespectra $\rho^{Y}$ is an isomorphism in all degrees. Since $\rho_{*}^{Y}$ on $\pi_{i}$ equals $\rho_{Y_{*}}$ on $\pi_{i+n}$, the fact that $\rho^{Y}$ is a weak equivalence immediately implies that $\rho_{Y}$ is also a weak equivalence.

Let $X_{\bullet}$ be a simplicial prespectrum. Assume that all the simplicial pointed spaces $X_{\bullet}^{k}$ are good and all the prespectra $X_{i}$ are $N$-connected. Then the prespectrum $\Sigma\left|X_{\bullet}\right|$ is $(N+1)$-connected, according to Corollary 2.10 and hence $\pi_{i}\left(\Sigma\left|X_{\bullet}\right|\right)=\pi_{i-1}\left(\left|X_{\bullet}\right|\right)$.

For every $n \geqslant 0$ we get a natural morphism of prespectra

$$
f i b\left(\operatorname{cube}_{n}\left(X_{\bullet}\right)\right) \stackrel{\rho}{\rightarrow} \Omega^{n}\left(\underset{n}{\operatorname{cofib}}\left(X_{\bullet}\right)\right) \stackrel{\eta}{\rightarrow} \Omega^{n}\left(\Sigma\left(\left|X_{\bullet}\right|\right)\right)
$$

and the induced homomorphism on homotopy groups

$$
\begin{aligned}
\pi_{i}\left(f i b\left(\operatorname{cube}_{n}\left(X_{\bullet}\right)\right)\right) & \left.\stackrel{\sim}{\rightarrow} \pi_{i}\left(\Omega^{n}\left(\operatorname{cofib}\left(\operatorname{cube}_{n}\left(X_{\bullet}\right)\right)\right)\right)=\pi_{i+n}\left(\operatorname{cofib} \operatorname{cube}_{n}\left(X_{\bullet}\right)\right)\right) \\
& \rightarrow \pi_{i+n}\left(\Sigma\left|X_{\bullet}\right|\right)=\pi_{i+n-1}\left(\left|X_{\bullet}\right|\right) .
\end{aligned}
$$

THEOREM 4.3.- Let $X_{\bullet}$ be a simplicial prespectrum. Assume that all the simplicial pointed spaces $X_{\bullet}^{k}$ are good and all the prespectra $X_{i}$ are $N$-connected. Then the natural homomorphism

$$
(\eta \circ \rho)_{*}: \pi_{i}\left(f i b\left(\operatorname{cube}_{n}\left(X_{\bullet}\right)\right)\right) \rightarrow \pi_{i+n-1}\left(\left|X_{\bullet}\right|\right)
$$

is an isomorphism in degrees $i \leqslant N$ (and an epimorphism in degree $N+1$ ). 
Proof. - Since the spaces $X_{i}^{k}$ are $(N+k)$-connected we conclude from Corollary 2.10 that the homomorphism

$$
\pi_{i+n+k}\left(\operatorname{cofib}\left(\operatorname{cube}_{n}\left(X_{\bullet}^{k}\right)\right)\right) \rightarrow \pi_{i+n+k}\left(\Sigma\left|X_{\bullet}^{k}\right|\right)
$$

is an isomorphism in degrees $\leqslant N+k+n$ (i.e., for $i \leqslant N$ ) and an epimorphism in degree $N+k+n+1$ (i.e., for $i=N+1$ ).

Proposition 4.2 and Theorem 4.3 easily imply the following useful result (whose last statement is certainly well known).

COROLLARY 4.4. - Let $f_{\bullet}: X_{\bullet} \rightarrow Y_{\bullet}$ be a morphism of $N$-connected simplicial prespectra. Assume that all the simplicial pointed spaces $X_{\bullet}^{k}, Y_{\bullet}^{k}$ are good. Assume further that each morphism of prespectra $f_{i}: X_{i} \rightarrow Y_{i}$ is a weak equivalence. Then

(1) For any $n \geqslant 0$ the induced morphism $f i b\left(\right.$ cube $\left._{n}\left(X_{\bullet}\right)\right) \rightarrow f i b\left(\right.$ cube $\left._{n}\left(Y_{\bullet}\right)\right)$ is a weak equivalence.

(2) For any $n \geqslant 0$ the induced morphism cofib $\left(\right.$ cube $\left._{n}\left(X_{\bullet}\right)\right) \rightarrow \operatorname{cofib}\left(\right.$ cube $\left._{n}\left(Y_{\bullet}\right)\right)$ is a weak equivalence.

(3) The induced morphism $\left|X_{\bullet}\right| \rightarrow\left|Y_{\bullet}\right|$ is a weak equivalence.

Proof. - The first statement is proved using immediate induction on $n$. The second follows from the first one and Proposition 4.2. The last statement follows from the first one and Theorem 4.3.

Once again, let $f_{\bullet}: X_{\bullet} \rightarrow Y_{\bullet}$ be a morphism of $N$-connected good simplicial prespectra. Denote by $\mathcal{F}_{\bullet}$ (respectively $\mathcal{C}_{\bullet}$ ) the homotopy fiber (respectively cofiber) of $f_{\bullet}$. Denote further by $\mathcal{F}$ the homotopy fiber of the morphism $\left|X_{\bullet}\right| \stackrel{\left|f_{\bullet}\right|}{\longrightarrow}\left|Y_{\bullet}\right|$. Note that we have obvious morphisms of prespectra

$$
\left|\mathcal{F}_{\bullet}\right| \rightarrow\left|X_{\bullet}\right|, \quad\left|\mathcal{F}_{\bullet}\right| \wedge \mathrm{I}=\left|\mathcal{F}_{\bullet} \wedge \mathrm{I}\right| \rightarrow\left|Y_{\bullet}\right|
$$

which are compatible and hence define a morphism

$$
\left|\mathcal{F}_{\bullet}\right| \rightarrow \mathcal{F}
$$

Here we consider I as a pointed space with distinguished point $1 \in \mathrm{I}$.

LEMMA 4.5. - The above defined morphism $\left|\mathcal{F}_{\bullet}\right| \rightarrow \mathcal{F}$ is a weak equivalence.

Proof. - Note that the simplicial spectra $\Sigma\left(\mathcal{F}_{\bullet}\right)$ and $\mathcal{C}_{\bullet}$ consist of good pointed simplicial spaces - see Corollary E.3. Our statement follows now from the commutativity of the diagram

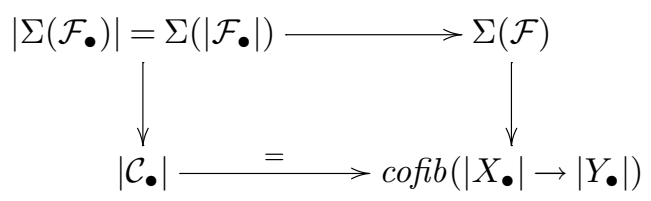

since both vertical arrows are weak equivalences, according to Corollary 4.4 and Proposition 4.2 respectively.

One might restate Lemma 4.5 by saying that the sequence

$$
\left|\mathcal{F}_{\bullet}\right| \rightarrow\left|X_{\bullet}\right| \rightarrow\left|Y_{\bullet}\right|
$$


is a homotopy fibration sequence. In particular we get canonical connecting homomorphisms

$$
\delta: \pi_{i}\left(\left|Y_{\bullet}\right|\right) \rightarrow \pi_{i-1}\left(\left|\mathcal{F}_{\bullet}\right|\right) .
$$

The following compatibility property will be essential in Section 6.

Proposition 4.6. - Let $f_{\bullet}: X_{\bullet} \rightarrow Y_{\bullet}$ be a morphism of $N$-connected good simplicial prespectra. Then the following diagram commutes (up to a sign) for any $n$.

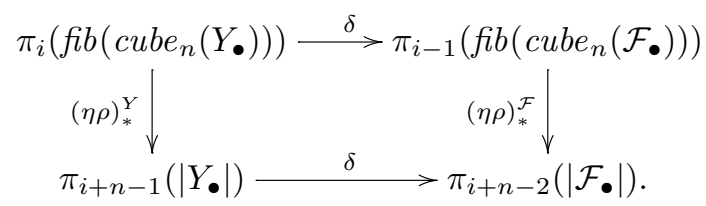

Proof. - To prove the statement we compose both maps $\pi_{i}\left(f i b\left(\operatorname{cube}_{n}\left(Y_{\bullet}\right)\right)\right) \rightarrow \pi_{i+n-2}\left(\left|\mathcal{F}_{\bullet}\right|\right)$ with the isomorphism $\pi_{i+n-2}\left(\left|\mathcal{F}_{\bullet}\right|\right) \stackrel{\sim}{\rightarrow} \pi_{i+n-1}\left(\left|\mathcal{C}_{\bullet}\right|\right)$. Using the above description of the connecting homomorphism $\delta: \pi_{*}\left(\left|Y_{\bullet}\right|\right) \rightarrow \pi_{*-1}\left(\mathcal{F}_{\bullet} \mid\right)$ one easily checks that the composition

$$
\pi_{i+n-1}\left(\left|Y_{\bullet}\right|\right) \stackrel{\delta}{\rightarrow} \pi_{i+n-2}\left(\left|\mathcal{F}_{\bullet}\right|\right) \stackrel{\sim}{\rightarrow} \pi_{i+n-1}\left(\left|\mathcal{C}_{\bullet}\right|\right)
$$

is induced by the obvious embedding $Y_{\bullet} \hookrightarrow \mathcal{C} \bullet$ and hence the composition of $\delta(\eta \rho)_{*}^{Y}$ with the above isomorphism may be also decomposed as

$$
\begin{aligned}
\pi_{i}\left(f i b\left(\operatorname{cube}_{n}\left(Y_{\bullet}\right)\right)\right) & \stackrel{\rho_{*}}{\longrightarrow} \pi_{i+n}\left(\operatorname{cofib}\left(\operatorname{cube}_{n}\left(Y_{\bullet}\right)\right)\right) \rightarrow \pi_{i+n}\left(\operatorname{cofib}\left(\operatorname{cube}_{n}\left(\mathcal{C}_{\bullet}\right)\right)\right) \\
\stackrel{\eta_{*}}{\longrightarrow} & \pi_{i+n-1}\left(\left|\mathcal{C}_{\bullet}\right|\right)
\end{aligned}
$$

On the other hand the commutative (up to a sign) diagram (see Corollary 2.12)

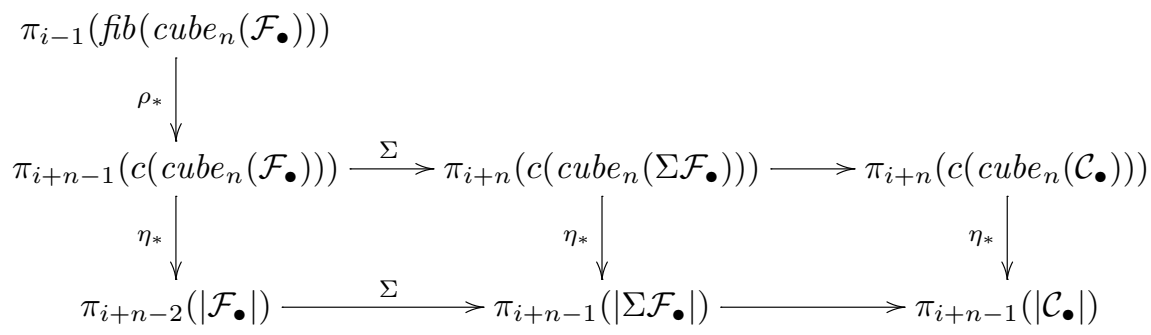

(here we had to abbreviate cofib to $c$ in the middle row) shows that the composition of $(\eta \rho)_{*} \delta$ with the isomorphism $\pi_{i+n-2}\left(\left|\mathcal{F}_{\bullet}\right|\right) \stackrel{\sim}{\rightarrow} \pi_{i+n-1}\left(\left|\mathcal{C}_{\bullet}\right|\right)$ coincides (up to a sign) with the composition

$$
\begin{aligned}
& \pi_{i}\left(f i b\left(\operatorname{cube}_{n}\left(Y_{\bullet}\right)\right)\right) \stackrel{\delta}{\rightarrow} \pi_{i-1}\left(f i b\left(\operatorname{cube}_{n}\left(\mathcal{F}_{\bullet}\right)\right)\right) \rightarrow \pi_{i+n-1}\left(\operatorname{cofib}\left(\operatorname{cube}_{n}\left(\mathcal{F}_{\bullet}\right)\right)\right)
\end{aligned}
$$

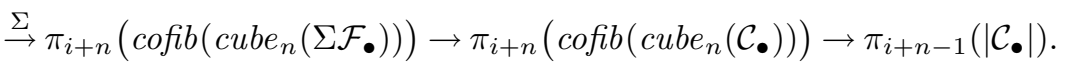

The proposition follows from these computations and Corollary 3.2.2.

Finally we mention the following result which follows immediately from Corollary 3.2.1. 
Lemma 4.7. - Let $X_{\bullet}$ be a $N$-connected simplicial prespectrum. Then the following diagram commutes up to a sign

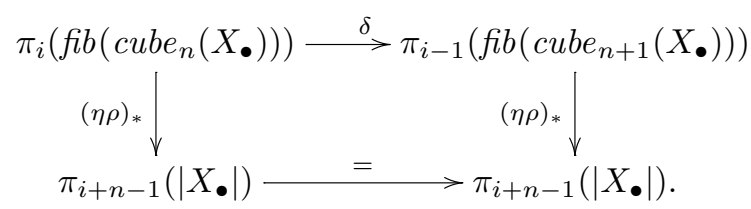

\section{Multirelative $K$-theory with supports}

A difficult theorem of Spencer Bloch and Steven Lichtenbaum, Theorem 5.5 below, asserts the exactness of a long exact sequence of multirelative $K_{0}$-groups associated to the $K$-theory of an arbitrary field $F$. This theorem is the starting point of our investigations. Bloch and Lichtenbaum's exact sequence provides them with an exact couple and thus a spectral sequence, the special case of a field of the spectral sequence we seek to construct. In Theorem 5.7, we provide a particularly useful interpretation of the derived exact couple of this exact couple in terms of homotopy groups of naturally defined simplicial prespectra.

As recalled in Appendix C.6, multirelative $K$-theory is essentially by definition the homotopy groups of appropriate iterated homotopy fibres of cubes of prespectra. We begin this section by recasting the Bloch-Lichtenbaum context of multirelative $K$-theory into our context of simplicial prespectra and associated iterated homotopy fibres. For example, Proposition 5.4 asserts that $\pi_{0}$ of an appropriate iterated homotopy fibre is the Moore complex associated to the Bloch complex, the complex whose homology groups are Bloch's higher Chow groups.

We fix a field $F$ and denote by $\Delta^{\bullet}$ the standard cosimplicial scheme over $F$ with $\Delta^{n}$ the standard algebraic $n$-simplex Spec $F\left[T_{0}, \ldots, T_{n}\right] /\left(T_{0}+\cdots+T_{n}-1\right)$. If $X$ is a smooth, irreducible scheme over $F$, we denote by $\Delta^{\bullet} \times X=\Delta^{\bullet} \times_{k} X$ the corresponding cosimplicial scheme over $X$.

For any $j, n \geqslant 0$, we let $\mathcal{C}^{j}(X, n)$ denote the family of closed subschemes $Y \subset \Delta^{n} \times X$ which meet every face of $\Delta^{n} \times X$ in codimension $\geqslant j$ (and in particular are of codimension $\geqslant j$ themselves). We shall employ the notation

$$
\mathcal{K}^{\mathcal{C}^{j}}\left(\Delta^{n} \times X\right) \equiv \mathcal{K}^{\mathcal{C}^{j}(X, n)}\left(\Delta^{n} \times X\right)
$$

for the algebraic $K$-theory prespectrum of coherent sheaves on $\Delta^{n} \times X$ with supports in $\mathcal{C}^{j}(X, n)$ (cf. Appendix C.6). Since each structure morphism of $\Delta^{\bullet}$ is a composition of a flat surjective morphism and a regular embedding, the inverse image of any $Y \in \mathcal{C}^{j}(X, n)$ under any structure morphism $\Delta^{m} \times X \rightarrow \Delta^{n} \times X$ belongs to $\mathcal{C}^{j}(X, m)$. As discussed in Appendix C.6, this implies that $n \mapsto \mathcal{K}^{\mathcal{C}^{j}}\left(\Delta^{n} \times X\right)$ is a simplicial prespectrum, which we denote by $\mathcal{K}^{\mathcal{C}^{j}}\left(\Delta^{\bullet} \times X\right)$. Whenever this can not lead to confusion, we use the same notation $\mathcal{K}^{\mathcal{C}^{j}}\left(\Delta^{\bullet} \times X\right)$ for the geometric realization of this simplicial prespectrum:

$$
\mathcal{K}^{\mathcal{C}^{j}}\left(\Delta^{\bullet} \times X\right) \equiv\left|n \mapsto \mathcal{K}^{\mathcal{C}^{j}}\left(\Delta^{n} \times X\right)\right|
$$

Following Bloch and Lichtenbaum, we consider multirelative $K$-theory. The key object of study is multirelative $K_{0}$ (with supports) of $\Delta^{n}$ with respect to all faces. Observe that the $(n+1)$-cube corresponding to the multirelative $K$-theory prespectrum of $\Delta^{n}$ with respect to all faces and with supports in $\mathcal{C}^{j} \equiv \mathcal{C}^{j}(\operatorname{Spec} F, n)$ is nothing but $c u b e_{n+1}\left(\mathcal{K}^{\mathcal{C}^{j}}\left(\Delta^{\bullet}\right)\right)$. Denoting 
this $(n+1)$-cube by $Y_{\bullet} \ldots \bullet$, one sees further that the $n$-cube corresponding to the prespectrum of multirelative $K$-theory of $\Delta^{n}$ with respect to all faces but the face given by the equation $t_{n}=0$ (and with supports in $\mathcal{C}^{j}$ ) coincides with $Y_{1}=Y_{\bullet}, \ldots, \bullet, 1$. Thus, denoting the family of all faces (of codimension one) of $\Delta^{n}$ by $\partial$ and the family of all faces except for the face given by the equation $t_{n}=0$ by $\Lambda$, we see that

$$
\begin{aligned}
& K_{0}^{\mathcal{C}^{j}}\left(\Delta^{n}, \partial\right)=\pi_{0}\left(\text { fib }\left(\text { cube }_{n+1}\left(\mathcal{K}^{\mathcal{C}^{j}}\left(\Delta^{\bullet}\right)\right)\right)\right), \\
& K_{0}^{\mathcal{C}^{j}}\left(\Delta^{n}, \Lambda\right)=\pi_{0}\left(f i b\left(\left\{\text { cube }_{n+1}\left(\mathcal{K}^{\mathcal{C}^{j}}\left(\Delta^{\bullet}\right)\right)\right\}_{1}\right)\right) .
\end{aligned}
$$

An easy but useful observation of Bloch and Lichtenbaum is that the multirelative $K$-theory of $\Delta^{n}$ with respect to all faces but one injects into the absolute $K$-theory and hence is easy to understand. Our first proposition recalls this result in slightly greater generality.

Proposition 5.1. - Let $X_{\bullet}$ be a simplicial prespectrum. Set $Y_{\bullet} \ldots \bullet=$ cube $_{n+1}\left(X_{\bullet}\right)$ and define the $n$-cube $Y_{1}=Y_{\bullet}, \ldots, \bullet, 1$ in the usual way. The homomorphisms of homotopy groups induced by the obvious projection $f i b\left(Y_{1}\right) \rightarrow Y_{1, \ldots, 1,1}=X_{n}$ are injective.

Proof. - For any $1 \leqslant k \leqslant n+1$ define the $(n+1-k)$-cube $Y_{k}$ by the formula

$$
Y_{k}=Y_{\bullet}, \ldots, \bullet \underbrace{1, \ldots, 1}_{k} .
$$

Thus $Y_{k+1}=\left(Y_{k}\right)_{1}$ and we have a homotopy fibration sequence

$$
f i b\left(Y_{k}\right) \rightarrow f i b\left(Y_{k+1}\right) \rightarrow f i b\left(\left(Y_{k}\right)_{0}\right)
$$

and hence an exact sequence of homotopy groups

$$
\pi_{i+1}\left(f i b\left(Y_{k+1}\right)\right) \rightarrow \pi_{i+1}\left(f i b\left(\left(Y_{k}\right)_{0}\right)\right) \rightarrow \pi_{i}\left(f i b\left(Y_{k}\right)\right) \rightarrow \pi_{i}\left(f i b\left(Y_{k+1}\right)\right) .
$$

We will see in a moment that the morphism $f i b\left(Y_{k+1}\right) \rightarrow f i b\left(\left(Y_{k}\right)_{0}\right)$ has a section and hence induces epimorphisms in homotopy groups. This together with the above exact sequence of homotopy groups implies that all homomorphisms $\pi_{i}\left(f i b\left(Y_{k}\right)\right) \rightarrow \pi_{i}\left(f i b\left(Y_{k+1}\right)\right.$ are (split) injective. Since $f i b\left(Y_{n+1}\right)=X_{n}$, our claim follows. The section in question is induced by the morphism of $(n-k)$-cubes $\left(Y_{k}\right)_{0} \rightarrow Y_{k+1}$

$$
\begin{aligned}
Y_{i_{0}, \ldots, i_{n-k-1}, 0,1, \ldots, 1} & =X_{i_{0}+\cdots+i_{n-k-1}+k-1} \stackrel{s_{i_{0}+\cdots+i_{n-k-1}}}{\longrightarrow} Y_{i_{0}, \ldots, i_{n-k-1}, 1,1, \ldots, 1} \\
& =X_{i_{0}+\cdots+i_{n-k-1}+k}
\end{aligned}
$$

(here $s_{\bullet}$ are the degeneracy operators of our simplicial prespectrum), which is left inverse to $d_{n-k}: Y_{k+1} \rightarrow\left(Y_{k}\right)_{0}$.

Proposition 5.1 has the following important corollary.

COROLlary 5.2. - With hypotheses and notation as in Proposition 5.1 the image of the canonical monomorphism

$$
\pi_{i}\left(f i b\left(\left\{\operatorname{cube}_{n+1}\left(X_{\bullet}\right)\right\}_{1}\right)\right) \hookrightarrow \pi_{i}\left(X_{n}\right)
$$

coincides with the nth term of the Moore complex $\mathcal{M}\left(\pi_{i}\left(X_{\bullet}\right)\right)$ corresponding to the simplicial abelian group $\pi_{i}\left(X_{\bullet}\right)$. In particular the prespectrum $f i b\left(\left\{\text { cube }_{n+1}\left(X_{\bullet}\right)\right\}_{1}\right)$ is $N$-connected provided that all prespectra $X_{i}$ are $N$-connected. 
Proof. - We show more generally that (in the notation of the proof of Proposition 5.1) for any $k$ the image of $\pi_{i}\left(f i b\left(Y_{k}\right)\right) \rightarrow \pi_{i}\left(X_{n}\right)$ coincides with the intersection of the kernels of face operations $\partial_{s}(0 \leqslant s \leqslant n-k)$ of the simplicial abelian group $\pi_{i}\left(X_{\bullet}\right)$. The statement is obvious for $k=n+1$. Proceeding by decreasing induction on $k$, we note that the image of $\pi_{i}\left(f i b\left(Y_{k}\right)\right) \rightarrow \pi_{i}\left(f i b\left(Y_{k+1}\right)\right)$ coincides with the kernel of the endomorphism of $\pi_{i}\left(f i b\left(Y_{k+1}\right)\right)$ induced by the endomorphism of the cube $Y_{k+1}$ :

$$
Y_{k+1} \stackrel{d_{n-k}}{\longrightarrow}\left\{Y_{k}\right\}_{0} \stackrel{s_{n-k}}{\longrightarrow} Y_{k+1}
$$

Since the endomorphism of $Y_{1, \ldots, 1}=X_{n}$ defined by the above endomorphism of the cube $Y_{k+1}$ equals $s_{n-k} \partial_{n-k}$, the statement follows.

We easily identify the differential in the Moore complex $\mathcal{M}\left(\pi_{i}\left(X_{\bullet}\right)\right)$.

COROLLARY 5.3. - With the identifications of Corollary 5.2, the homomorphism in homotopy groups induced by the following composition of morphisms of prespectra

$$
f i b\left(\left\{\operatorname{cube}_{n+1}\left(X_{\bullet}\right)\right\}_{1}\right) \rightarrow f i b\left(\left\{c u b e_{n+1}\left(X_{\bullet}\right)\right\}_{0}\right)=f i b\left(\left\{c u b e_{n}\left(X_{\bullet}\right)\right\}\right) \rightarrow f i b\left(\left\{c u b e_{n}\left(X_{\bullet}\right)\right\}_{1}\right)
$$

coincides with the differential $\partial_{n}$ of the Moore complex.

Proof. - This follows immediately from the commutativity of the diagram

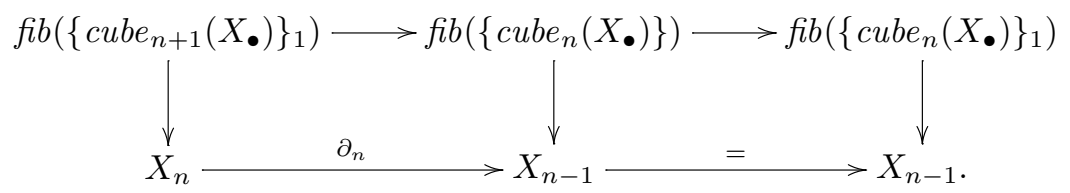

We next recall the definition of the higher Chow groups of Bloch [3]. Let $X$ be an equidimensional scheme of finite type over a field $F$. Let $z^{q}(X, n)$ be the free abelian group generated by closed integral subschemes $Z \subset \Delta^{n} \times X$ of codimension $q$ which intersect all faces of $\Delta^{n} \times X$ properly. One checks easily that $z^{q}(X, \bullet)$ is a simplicial abelian group and one defines Bloch's higher Chow groups as $C H^{q}(X, n)=\pi_{n}\left(z^{q}(X, \bullet)\right)$.

Another useful application of Proposition 5.1 is the following.

PROPOSITION 5.4 (cf. [5]). - The prespectrum fib $\left(\left\{\text { cube }{ }_{n+1}\left(\mathcal{K}^{\mathcal{C}^{q}}\left(\Delta^{\bullet}\right)\right)\right\}_{1}\right)$ is $(-1)$-connected. The group $\pi_{0}\left(f i b\left(\left\{\text { cube } e_{n+1}\left(\mathcal{K}^{\mathcal{C}^{q}}\left(\Delta^{\bullet}\right)\right)\right\}_{1}\right)\right)=K_{0}^{\mathcal{C}^{q}}\left(\Delta^{n}, \Lambda\right)$ coincides with the th term of the Moore complex $\mathcal{M}^{q}(F, \bullet)=\mathcal{M}\left(z^{q}(F, \bullet)\right)$ corresponding to the simplicial abelian group $z^{q}(F, \bullet)$.

Proof. - In view of Corollary 5.2 it suffices to establish that $K_{0}^{\mathcal{C}^{q}}\left(\Delta^{n}\right)$ is canonically isomorphic to $z^{q}(F, n)$ via the map which sends a coherent sheaf on $\Delta^{n}$ to its support. This is proved in $[15, \S 1]$ (see also [5]); the essential point is that the natural map

$$
K_{0}^{\mathcal{C}^{q+1}}\left(\Delta^{n}\right) \rightarrow K_{0}^{\mathcal{C}^{q}}\left(\Delta^{n}\right)
$$

is 0 .

Here is the fundamental exact sequence established by Bloch and Lichtenbaum which plays the central role in what follows. 
THEOREM 5.5 [5]. - The following sequence is exact

$$
\begin{aligned}
\cdots \rightarrow K_{0}^{\mathcal{C}^{q+1}}\left(\Delta^{n}, \partial\right) & \stackrel{i}{\rightarrow} K_{0}^{\mathcal{C}^{q}}\left(\Delta^{n}, \partial\right) \stackrel{j}{\rightarrow} K_{0}^{\mathcal{C}^{q}}\left(\Delta^{n}, \Lambda\right)=\mathcal{M}^{q}(F, n) \stackrel{k}{\rightarrow} K_{0}^{\mathcal{C}^{q}}\left(\Delta^{n-1}, \partial\right) \\
& \stackrel{i}{\rightarrow} K_{0}^{\mathcal{C}^{q-1}}\left(\Delta^{n}, \partial\right) \rightarrow \cdots .
\end{aligned}
$$

Here the first arrow is induced by the obvious embedding of the families of supports $\mathcal{C}^{q+1} \subset \mathcal{C}^{q}$, the second and the third arrows come from the long homotopy sequence corresponding to the fibration

$$
\begin{aligned}
f i b\left(\operatorname{cube}_{n+1}\left(\mathcal{K}^{\mathcal{C}^{q}}\left(\Delta^{\bullet}\right)\right)\right) & \rightarrow f i b\left(\left\{\operatorname{cube}_{n+1}\left(\mathcal{K}^{\mathcal{C}^{q}}\left(\Delta^{\bullet}\right)\right)\right\}_{1}\right) \\
& \rightarrow f i b\left(\left\{\operatorname{cube}_{n+1}\left(\mathcal{K}^{\mathcal{C}^{q}}\left(\Delta^{\bullet}\right)\right)\right\}_{0}\right)=f i b\left(\operatorname{cube}_{n}\left(\mathcal{K}^{\mathcal{C}^{q}}\left(\Delta^{\bullet}\right)\right)\right) .
\end{aligned}
$$

Theorem 5.5 gives immediately an exact couple

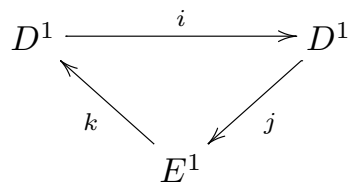

with $D_{p, q}^{1}=K_{0}^{\mathcal{C}^{q}}\left(\Delta^{p+q}, \partial\right)$ and $E_{p, q}^{1}=\mathcal{M}^{q}(F, p+q)$ and hence a spectral sequence converging to the algebraic $K$-theory of $F$. The differential of the complex $\mathcal{M}^{q}(F, \bullet)$ is the standard one by Corollary 5.3 and hence the Bloch-Lichtenbaum spectral sequence has the form

$$
E_{p, q}^{2}=C H^{q}(F, p+q) \Rightarrow K_{p+q}(F) .
$$

Changing signs, we may re-index this spectral sequence cohomologically as follows:

$$
E_{2}^{p, q}=C H^{-q}(F,-p-q) \Rightarrow K_{-p-q}(F) .
$$

The key to our generalization of this spectral sequence to smooth varieties $X$ over $F$ is the following observation which identifies the derived exact couple of the Bloch-Lichtenbaum exact couple in a useful way. Recall that the term $D^{2}$ of an exact couple equals the image of $i: D^{1} \rightarrow D^{1}$ and hence is naturally isomorphic to the cokernel of $k: E^{1} \rightarrow D^{1}$. Thus

$$
D_{p, q}^{2}=\operatorname{Coker}\left(K_{0}^{\mathcal{C}^{q+1}}\left(\Delta^{p+q+1}, \Lambda\right) \stackrel{k}{\rightarrow} K_{0}^{\mathcal{C}^{q+1}}\left(\Delta^{p+q}, \partial\right)\right) .
$$

To compute the above cokernel we consider the exact homotopy sequence of the fibration

$$
\begin{aligned}
& f i b\left(\operatorname{cube}_{p+q+2}\left(\mathcal{K}^{\mathcal{C}^{q+1}}\left(\Delta^{\bullet}\right)\right)\right) \\
& \quad \rightarrow f i b\left(\left\{\operatorname{cube}_{p+q+2}\left(\mathcal{K}^{\mathcal{C}^{q+1}}\left(\Delta^{\bullet}\right)\right)\right\}_{1}\right) \\
& \quad \rightarrow f i b\left(\left\{\operatorname{cube}_{p+q+2}\left(\mathcal{K}^{\mathcal{C} q+1}\left(\Delta^{\bullet}\right)\right)\right\}_{0}\right)=f i b\left(\text { cube }_{p+q+1}\left(\mathcal{K}^{\mathcal{C}^{q+1}}\left(\Delta^{\bullet}\right)\right)\right)
\end{aligned}
$$

whose relevant part is

$$
K_{0}^{\mathcal{C}^{q+1}}\left(\Delta^{p+q+1}, \Lambda\right) \stackrel{k}{\rightarrow} K_{0}^{\mathcal{C}^{q+1}}\left(\Delta^{p+q}, \partial\right) \stackrel{\delta}{\rightarrow} \pi_{-1}\left(f i b\left(c u b e_{p+q+2}\left(\mathcal{K}^{\mathcal{C}^{q+1}}\left(\Delta^{\bullet}\right)\right)\right)\right) \rightarrow 0 .
$$

(The exactness at the far right is a consequence of Corollary 5.2.) Theorem 4.3 (with $N=-1$, $i=-1, n=p+q+2$ ) now identifies the cokernel of $k$, thereby establishing the following statement. 
THEOREM 5.7. - We have a natural identification

$$
D_{p, q}^{2}=K_{p+q}^{\mathcal{C}^{q+1}}\left(\Delta^{\bullet}\right) \equiv \pi_{p+q}\left(\left|\mathcal{K}^{\mathcal{C}^{q+1}}\left(\Delta^{\bullet}\right)\right|\right)
$$

\section{Topological filtration for the $K$-theory of $\Delta^{\bullet}$}

Let $X$ be an irreducible smooth scheme of finite type over $F$. The homotopy invariance of algebraic $K$-theory implies readily that the obvious morphism of prespectra $\mathcal{K}(X) \rightarrow \mathcal{K}\left(\Delta^{\bullet} \times X\right)$ is a weak equivalence (cf. Proposition B.1). In generalizing the spectral sequence (5.6.0) from the special case $X=\operatorname{Spec} F$ to such a smooth $X$, we shall employ the spectral sequence associated to the following tower of prespectra (see Proposition 6.1 below)

$$
\mathcal{K}\left(\Delta^{\bullet} \times X\right)=\mathcal{K}^{\mathcal{C}^{0}}\left(\Delta^{\bullet} \times X\right) \leftarrow \mathcal{K}^{\mathcal{C}^{1}}\left(\Delta^{\bullet} \times X\right) \leftarrow \mathcal{K}^{\mathcal{C}^{2}}\left(\Delta^{\bullet} \times X\right) \leftarrow \cdots
$$

Our eventual goal is to show that the spectral sequence associated to this tower strongly converges to the $K$-theory of $X$ and has $E_{p, q}^{2}$-groups given by the higher Chow groups of $X$. To prove this, we must identify the homotopy fiber of each morphism $\mathcal{K}^{\mathcal{C}^{q}}\left(\Delta^{\bullet} \times X\right) \leftarrow \mathcal{K}^{\mathcal{C}^{q+1}}\left(\Delta^{\bullet} \times X\right)$.

In this section, we begin this task by investigating the special case in which $X=\operatorname{Spec} F$, the case in which the Bloch-Lichtenbaum Theorem (Theorem 5.5) provides us with the strongly convergent spectral sequence (5.6.0). The import of Theorem 6.2 is that it shows that the BlochLichtenbaum spectral sequence (5.6.0) arises as the spectral sequence of the tower (6.0) (for $X=\operatorname{Spec} F$ ).

PROPOSITION 6.1. - Consider a sequence of pointed maps of connected spaces with abelian fundamental groups

$$
\cdots \stackrel{f_{q+2}}{\longrightarrow} X_{q+1} \stackrel{f_{q+1}}{\longrightarrow} X_{q} \stackrel{f_{q}}{\longrightarrow} \cdots \stackrel{f_{1}}{\longrightarrow} X_{0}=X .
$$

Assume further that for each $q$ we are given a pointed map $p_{q}: X_{q} \rightarrow B_{q}$ with $B_{q}$ connected such that the composition $X_{q+1} \rightarrow X_{q} \rightarrow B_{q}$ is trivial and the associated map from $X_{q+1}$ to the homotopy fiber of $X_{q} \rightarrow B_{q}$ is a weak equivalence. Assume further that for each $i \geqslant 0$ there exists $n \geqslant 0$ such that $X_{q}$ is $i$-connected for $q \geqslant n$. In this case there exists a strongly convergent spectral sequence

$$
E_{p q}^{2}=\pi_{p+q}\left(B_{q}\right) \Longrightarrow \pi_{p+q}(X) .
$$

Proof. - Set $D_{p q}^{2}=\pi_{p+q}\left(X_{q}\right), \quad E_{p q}^{2}=\pi_{p+q}\left(B_{q}\right)$. Considering long exact homotopy sequences corresponding to the homotopy fibration sequences

$$
X_{q+1} \rightarrow X_{q} \rightarrow B_{q}
$$

we conclude that $\left(D^{2}, E^{2}\right)$ is an exact couple (with maps $i, j, k$ of bidegrees $(1,-1),(0,0)$, $(-2,1)$ respectively) and hence defines a spectral sequence. The assumption concerning high connectivity of $X_{q}$ implies that the exact couple is bounded below (i.e., for any $n$ there exists $f(n)$ such that $D_{p q}^{2}=0$ whenever $p<f(p+q)$ ). Since the exact couple is obviously bounded above we conclude that the spectral sequence converges to $H_{n}=\lim _{p} D_{p, n-p}^{2}=\pi_{n}(X)$ - see $[38, \S 5.9]$.

The previous proposition admits an obvious generalization to the case of prespectra 
COROLLARY 6.1.1. - Consider a sequence of maps of prespectra

$$
\cdots \stackrel{f_{q+2}}{\longrightarrow} X_{q+1} \stackrel{f_{q+1}}{\longrightarrow} X_{q} \stackrel{f_{q}}{\longrightarrow} \cdots \stackrel{f_{1}}{\longrightarrow} X_{0}=X .
$$

Assume further that for each $q$ we are given a map of prespectra $p_{q}: X_{q} \rightarrow B_{q}$ such that the composition $X_{q+1} \rightarrow X_{q} \rightarrow B_{q}$ is trivial and the associated map from $X_{q+1}$ to the homotopy fiber of $X_{q} \rightarrow B_{q}$ is a weak equivalence. Assume further that for each $i \geqslant 0$ there exists $n \geqslant 0$ such that $X_{q}$ is $i$-connected for $q \geqslant n$. In this case there exists a strongly convergent spectral sequence

$$
E_{p q}^{2}=\pi_{p+q}\left(B_{q}\right) \quad \Rightarrow \quad \pi_{p+q}(X) .
$$

Assume in addition that all $X_{i}$ and $B_{i}$ are $(-1)$-connected $\Omega$-prespectra. Then for every $n \geqslant 1$ the tower of connected spaces with abelian fundamental groups

$$
\cdots \stackrel{f_{q+2}^{n}}{\longrightarrow} X_{q+1}^{n} \stackrel{f_{q+1}^{n}}{\longrightarrow} X_{q}^{n} \stackrel{f_{q}^{n}}{\longrightarrow} \cdots \stackrel{f_{1}^{n}}{\longrightarrow} X_{0}^{n}=X^{n}
$$

and maps $p_{q}^{n}: X_{q}^{n} \rightarrow B_{q}^{n}$ satisfy the conditions of Proposition 6.1 and hence define a spectral sequence, which coincides (up to a shift of degrees) with the spectral sequence defined by the original tower of prespectra.

Hence, to obtain a useful spectral sequence from the tower (6.0) we must identify the homotopy fibres of the maps

$$
\mathcal{K}^{\mathcal{C}^{q+1}}\left(\Delta^{\bullet} \times X\right) \rightarrow \mathcal{K}^{\mathcal{C}^{q}}\left(\Delta^{\bullet} \times X\right)
$$

The following theorem achieves such an identification for $X=\operatorname{Spec} F$.

THEOREM 6.2. - For any $q \geqslant 0$ the sequence of maps of $\Omega$-prespectra

$$
\mathcal{K}^{\mathcal{C}^{q+1}}\left(\Delta^{\bullet}\right) \rightarrow \mathcal{K}^{\mathcal{C}^{q}}\left(\Delta^{\bullet}\right) \rightarrow\left|\mathbb{B}\left(z^{q}(F, \bullet)\right)\right|
$$

is a homotopy fibration sequence. Here, $\mathcal{K}^{\mathcal{C}^{q}}\left(\Delta^{\bullet}\right) \rightarrow\left|\mathbb{B}\left(z^{q}(F, \bullet)\right)\right|$ is the canonical morphism of prespectra of $(\mathrm{C} .1 .1)$, and the canonical map from $\mathcal{K}^{\mathcal{C}^{q+1}}\left(\Delta^{\bullet}\right)$ to the homotopy fiber of $\mathcal{K}^{\mathcal{C}^{q}}\left(\Delta^{\bullet}\right) \rightarrow\left|\mathbb{B}\left(z^{q}(F, \bullet)\right)\right|$ comes from the fact that the composition morphism

$$
\mathcal{K}^{\mathcal{C}^{q+1}}\left(\Delta^{\bullet}\right) \rightarrow \mathcal{K}^{\mathcal{C}^{q}}\left(\Delta^{\bullet}\right) \rightarrow \mathbb{B}\left(z^{q}(F, \bullet)\right)
$$

is trivial.

Proof (depending upon Lemma 6.3 and Propositions 6.5, 6.6 below). - Let $\mathcal{F}^{q}\left(\Delta^{n}\right)$ denote the homotopy fiber of the map of prespectra $\mathcal{K}^{\mathcal{C}^{q}}\left(\Delta^{n}\right) \rightarrow \mathbb{B}\left(z^{q}(F, n)\right)$,

$$
\mathcal{F}^{q}\left(\Delta^{n}\right) \equiv f i b\left(\mathcal{K}^{\mathcal{C}^{q}}\left(\Delta^{n}\right) \rightarrow \mathbb{B}\left(z^{q}(F, n)\right)\right) .
$$

The prespectra $\mathcal{F}^{q}\left(\Delta^{n}\right)$ obviously form a simplicial prespectrum. Lemma 4.5 implies that the canonical morphism of prespectra

$$
\left|\mathcal{F}^{q}\left(\Delta^{\bullet}\right)\right| \rightarrow f i b\left(\left|n \mapsto \mathcal{K}^{\mathcal{C}^{q}}\left(\Delta^{n}\right)\right| \rightarrow\left|n \mapsto \mathbb{B}\left(z^{q}(F, n)\right)\right|\right)
$$


is a weak equivalence. Thus, the sequence

$$
\left|\mathcal{F}^{q}\left(\Delta^{\bullet}\right)\right| \rightarrow \mathcal{K}^{\mathcal{C}^{q}}\left(\Delta^{\bullet}\right)=\left|n \mapsto \mathcal{K}^{\mathcal{C}^{q}}\left(\Delta^{n}\right)\right| \rightarrow\left|n \mapsto \mathbb{B}\left(z^{q}(F, n)\right)\right|
$$

is a homotopy fibration sequence.

For each $n$ the composition morphism

$$
\mathcal{K}^{\mathcal{C}^{q+1}}\left(\Delta^{n}\right) \rightarrow \mathcal{K}^{\mathcal{C}^{q}}\left(\Delta^{n}\right) \rightarrow \mathbb{B}\left(z^{q}(F, n)\right)
$$

is trivial and hence defines a canonical morphism of prespectra $\mathcal{K}^{\mathcal{C}^{q+1}}\left(\Delta^{n}\right) \rightarrow \mathcal{F}^{q}\left(\Delta^{n}\right)$. We proceed to show that the resulting map on geometric realizations

$$
\mathcal{K}^{\mathcal{C}^{q+1}}\left(\Delta^{\bullet}\right)=\left|n \mapsto \mathcal{K}^{\mathcal{C}^{q+1}}\left(\Delta^{n}\right)\right| \rightarrow \mathcal{F}^{q}\left(\Delta^{\bullet}\right)=\left|n \mapsto \mathcal{F}^{q}\left(\Delta^{n}\right)\right|
$$

is also a weak equivalence. To do so we compare the exact sequence of the derived exact couple $\left(D^{2}, E^{2}\right)$ with the long exact sequence of homotopy groups for the homotopy fibration (6.2.1). Thus we consider the diagram (in which $n=p+q$ )

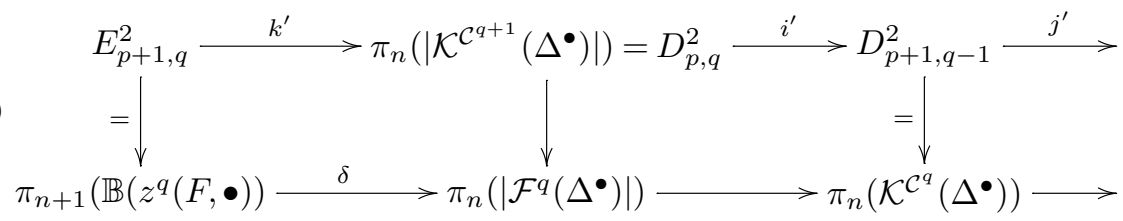

In the remainder of this section, we verify the commutativity of (6.2.3) which will, by the 5-Lemma, imply that (6.2.2) is a homotopy equivalence.

To prove the commutativity of the above diagram we identify the homomorphisms $i^{\prime}, j^{\prime}, k^{\prime}$ appearing in the derived exact couple and check the commutativity of each of the three types of squares in this ladder. This is achieved in Lemma 6.3, Proposition 6.5, and Proposition 6.6.

The naturality of our identification in Theorem 5.7 immediately implies the following lemma identifying $i^{\prime}$ and proving part of the required commutativity of (6.2.3).

LEMMA 6.3. - The homomorphism

$$
i^{\prime}: D_{p, q}^{2}=K_{p+q}^{\mathcal{C}^{q+1}}\left(\Delta^{\bullet}\right) \rightarrow D_{p+1, q-1}^{2}=K_{p+q}^{\mathcal{C}^{q}}\left(\Delta^{\bullet}\right)
$$

is induced by the canonical morphism of prespectra $\mathcal{K}^{\mathcal{C}^{q+1}}\left(\Delta^{\bullet}\right) \rightarrow \mathcal{K}^{\mathcal{C}^{q}}\left(\Delta^{\bullet}\right)$.

Next we identify the homomorphism

$$
j^{\prime}: D_{p+1, q-1}^{2}=\pi_{p+q}\left(\mathcal{K}^{\mathcal{C}^{q}}\left(\Delta^{\bullet}\right)\right) \rightarrow E_{p, q}^{2}=C H^{q}(F, p+q)=\pi_{p+q}\left(\mathbb{B}\left(z^{q}(F, \bullet)\right)\right) .
$$

Before doing so we discuss a certain general construction which applies to any $(-1)$-connected good simplicial prespectrum and of which the homomorphism $j^{\prime}$ is a special case. So let $X \bullet$ be a $(-1)$-connected good simplicial prespectrum. Consider the diagram whose column is exact by Corollary 5.2 


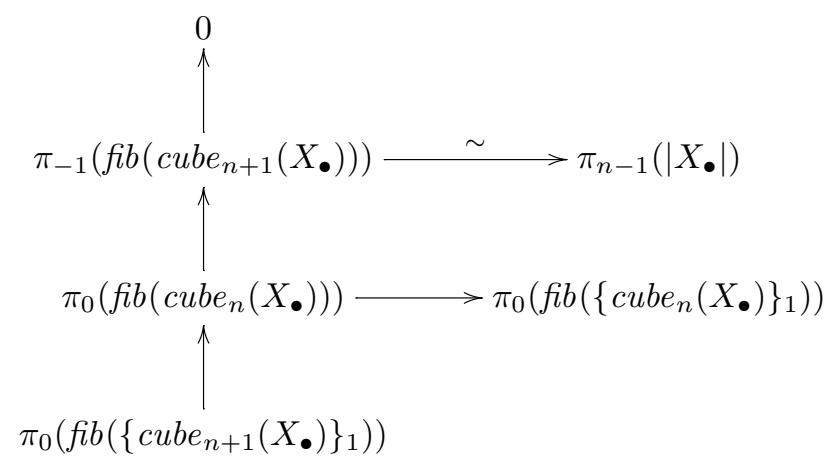

The isomorphism in (6.3.1) is a consequence of Theorem 4.3. Start with an element in $\pi_{n-1}\left(\left|X_{\bullet}\right|\right)$, lift it to $\pi_{0}\left(f i b\left(\operatorname{cube}_{n}\left(X_{\bullet}\right)\right)\right)$ and then take the image of the lifting in $\pi_{0}\left(f i b\left(\left\{\operatorname{cube}_{n}\left(X_{\bullet}\right)\right\}_{1}\right)\right)=\mathcal{M}_{n-1}\left(\pi_{0}\left(X_{\bullet}\right)\right)$. One checks immediately that the resulting element is a cycle of the complex $\mathcal{M}\left(\pi_{0}\left(X_{\bullet}\right)\right)$ and the homology class of this cycle is independent of the choice of the lifting. Thus for any $(-1)$-connected good simplicial prespectrum $X \bullet$ we get a canonical homomorphism, which we denote $j^{\prime}{ }_{X}$

$$
j_{X}^{\prime}: \pi_{n-1}\left(\left|X_{\bullet}\right|\right) \rightarrow H_{n-1}\left(\mathcal{M}\left(\pi_{0}\left(X_{\bullet}\right)\right)\right)=\pi_{n-1}\left(\pi_{0}\left(X_{\bullet}\right)\right) .
$$

This homomorphism is obviously functorial with respect to morphisms of $(-1)$-connected good simplicial prespectra and the homomorphism

$$
j^{\prime}: D_{p, q}^{2}=\pi_{p+q}\left(\mathcal{K}^{\mathcal{C}^{q}}\left(\Delta^{\bullet}\right)\right) \rightarrow E_{p, q}^{2}=C H^{q}(F, p+q)=\pi_{p+q}\left(z^{q}(F, \bullet)\right)
$$

from the exact couple $\left(D^{2}, E^{2}\right)$ is exactly this homomorphism applied to the simplicial prespectrum $\mathcal{K}^{\mathcal{C}^{q}}\left(\Delta^{\bullet}\right)$.

One function of the isomorphism established in the following lemma is to make explicit the identification between $E_{p, q}^{2}=\pi_{p+q}\left(z^{q}(F, \bullet)\right)$ and $\pi_{p+q}\left(\mathbb{B}\left(z^{q}(F, \bullet)\right)\right)$.

LEMmA 6.4. - Let $A_{\bullet}$ be a simplicial abelian group and let $X_{\bullet}=\mathbb{B}\left(A_{\bullet}\right)$ be the corresponding simplicial prespectrum. The corresponding homomorphism

$$
\pi_{n-1}\left(\left|X_{\bullet}\right|\right) \stackrel{j_{X}^{\prime}}{\longrightarrow} \pi_{n-1}\left(\pi_{0}\left(X_{\bullet}\right)\right)
$$

is an isomorphism for any $n$.

Proof. - Note that $\pi_{i}\left(X_{n}\right)=0$ for all $n$ and all $i \neq 0$. In view of Proposition 5.1 this readily implies that $\pi_{i}\left(f i b\left(\right.\right.$ cube $\left.\left._{n+1}\left(X_{\bullet}\right)_{1}\right)\right)=0$ for all $n$ and all $i \neq 0$. Using now an easy induction on $n$ we conclude that

$$
\pi_{i}\left(f i b\left(\operatorname{cube}_{n}\left(X_{\bullet}\right)\right)\right)=0, \quad \forall n, i>0,
$$

This vanishing of homotopy groups in positive degrees implies further the injectivity (for all $n$ ) of the homomorphism

$$
\pi_{0}\left(f i b\left(\operatorname{cube}_{n}\left(X_{\bullet}\right)\right)\right) \rightarrow \pi_{0}\left(f i b\left(\left\{\operatorname{cube}_{n}\left(X_{\bullet}\right)\right\}_{1}\right)\right)=\mathcal{M}_{n-1}\left(\pi_{0}\left(X_{\bullet}\right)\right) .
$$

Next we extend the middle row of (6.3.1) to the exact sequence 


$$
\begin{aligned}
0 & =\pi_{1}\left(f i b\left(\left\{\operatorname{cube}_{n}\left(X_{\bullet}\right)\right\}_{0}\right)\right) \\
& \rightarrow \pi_{0}\left(f i b \left(\pi _ { 0 } \left(f i b\left(\left\{c u b e_{n}\left(X_{\bullet}\right)\right)\right)\right.\right.\right. \\
\left.\left.\left.\left.X_{\bullet}\right)\right\}_{1}\right)\right) & \rightarrow \pi_{0}\left(f i b\left(\left\{c u b e_{n}\left(X_{\bullet}\right)\right\}_{0}\right)\right) .
\end{aligned}
$$

The above remarks together with Corollary 5.3 show that cycles in degree $n-1$ of the Moore complex $\mathcal{M}\left(\pi_{0}\left(X_{\bullet}\right)\right)$ coincide with the kernel of the last map. This implies the surjectivity of $j_{X}^{\prime}$. Injectivity of $j_{X}^{\prime}$ follows immediately from the injectivity of

$$
\pi_{0}\left(f i b\left(\operatorname{cube}_{n}\left(X_{\bullet}\right)\right)\right) \rightarrow \pi_{0}\left(f i b\left(\left\{\operatorname{cube}_{n}\left(X_{\bullet}\right)\right\}_{1}\right)\right) .
$$

Lemma 6.4 enables us to identify the homomorphism $j^{\prime}$ and verify another portion of the commutativity of (6.2.3).

PROPOSITION 6.5. - The homomorphism

$$
j^{\prime}: D_{p+1, q-1}^{2}=\pi_{p+q}\left(\mathcal{K}^{\mathcal{C}^{q}}\left(\Delta^{\bullet}\right)\right) \rightarrow E_{p, q}^{2}=C H^{q}(F, p+q)=\pi_{p+q}\left(\mathbb{B}\left(z^{q}(F, \bullet)\right)\right)
$$

coincides with the canonical map in homotopy groups induced by the morphism of simplicial prespectra $\mathcal{K}^{\mathcal{C}^{q}}\left(\Delta^{\bullet}\right) \rightarrow \mathbb{B}\left(z^{q}(F, \bullet)\right)$.

Proof. - We use the isomorphism of Lemma 6.4 to identify $E_{p, q}^{2}=\pi_{p+q}\left(z^{q}(F, \bullet)\right)$ with $\pi_{p+q}\left(\mathbb{B}\left(z^{q}(F, \bullet)\right)\right)$. Then, our statement follows from the commutativity of the diagram

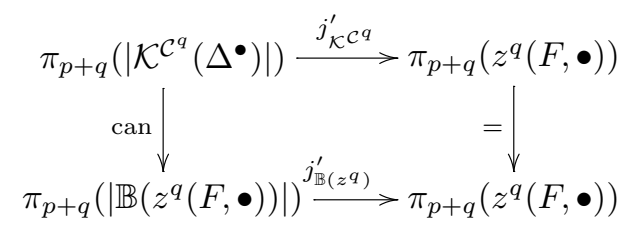

together with the verification achieved prior to Lemma 6.4 that $j^{\prime}=j^{\prime} \mathcal{K}^{\mathcal{C}^{q}}$.

Finally we need to identify the homomorphism (where as always $n=p+q$ )

$$
k^{\prime}: E_{p, q}^{2}=\pi_{n}\left(\left|\mathbb{B}\left(z^{q}(F, \bullet)\right)\right|\right) \rightarrow D_{p-1, q}^{2}=\pi_{n-1}\left(\left|\mathcal{K}^{\mathcal{C}^{q+1}}\left(\Delta^{\bullet}\right)\right|\right)
$$

or rather its composition with the homomorphism

$$
\pi_{n-1}\left(\left|\mathcal{K}^{\mathcal{C}^{q+1}}\left(\Delta^{\bullet}\right)\right|\right) \rightarrow \pi_{n-1}\left(\left|\mathcal{F}^{q}\left(\Delta^{\bullet}\right)\right|\right) .
$$

PROPOSITION 6.6. - The following diagram commutes (up to a sign)

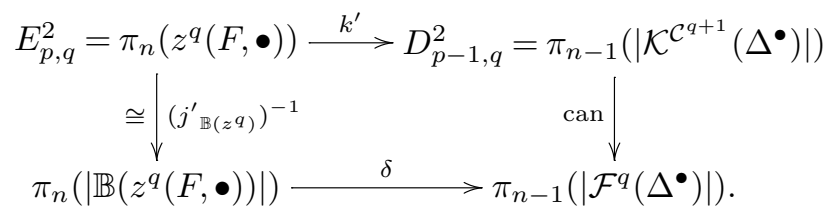

Here $\delta$ is the connecting homomorphism from the long exact homotopy sequence corresponding to the homotopy fibration sequence

$$
\left|\mathcal{F}^{q}\left(\Delta^{\bullet}\right)\right| \rightarrow\left|\mathcal{K}^{\mathcal{C}^{q}}\left(\Delta^{\bullet}\right)\right| \rightarrow\left|\mathbb{B}\left(z^{q}(F, \bullet)\right)\right| .
$$


Proof. - The homomorphism $k^{\prime}$ originates from the diagram

$$
\begin{gathered}
\pi_{n-1}\left(\left|\mathcal{K}^{\mathcal{C}^{q+1}}\left(\Delta^{\bullet}\right)\right|\right) \\
(\eta \rho)_{*} \uparrow \cong \\
\pi_{-1}\left(f i b\left(\operatorname{cube}_{n+1}\left(\mathcal{K}^{\mathcal{C}^{q+1}}\left(\Delta^{\bullet}\right)\right)\right) \stackrel{\delta}{\longrightarrow} \pi_{0}\left(f i b\left(\operatorname{cube}_{n}\left(\mathcal{K}^{\mathcal{C}^{q+1}}\left(\Delta^{\bullet}\right)\right)\right)\right)\right. \\
\pi_{0}\left(f i b\left(\left\{c u b e_{n+1}\left(\mathcal{K}^{\mathcal{C}^{q}}\left(\Delta^{\bullet}\right)\right)\right\}_{1}\right)\right) \stackrel{k}{i} \pi_{0}\left(f i b\left(\operatorname{cube}_{n}\left(\mathcal{K}^{\mathcal{C}^{q}}\left(\Delta^{\bullet}\right)\right)\right)\right) \\
j \mid \\
\pi_{0}\left(f i b\left(\left\{\operatorname{cube}_{n}\left(\mathcal{K}^{\mathcal{C}^{q}}\left(\Delta^{\bullet}\right)\right)\right\}_{1}\right)\right)
\end{gathered}
$$

Namely, we start with a $n$-cycle $x$ of the complex

$$
\pi_{0}\left(f i b\left(\left\{\operatorname{cube}_{*+1}\left(\mathcal{K}^{\mathcal{C}^{q}}\left(\Delta^{\bullet}\right)\right)\right\}_{1}\right)\right)=\mathcal{M}\left(z^{q}(F, *)\right) .
$$

Since $j \circ k(x)=0$ we can find $y \in \pi_{0}\left(f i b\left(c u b e_{n}\left(\mathcal{K}^{\mathcal{C}^{q+1}}\left(\Delta^{\bullet}\right)\right)\right)\right)$ such that $i(y)=k(x)$ and we set $k^{\prime}([x])=(\eta \rho)_{*} \delta(y)$. To compute the image of $k^{\prime}([x])$ in $\pi_{n-1}\left(\mid \mathcal{F}^{q}\left(\Delta^{\bullet} \mid\right)\right.$ we may take the image $z$ of $y$ in $\pi_{0}\left(f i b\left(\operatorname{cube}_{n}\left(\mathcal{F}^{q}\left(\Delta^{\bullet}\right)\right)\right)\right)$ and then apply the homomorphism

$$
\pi_{0}\left(f i b\left(\operatorname{cube}_{n}\left(\mathcal{F}^{q}\left(\Delta^{\bullet}\right)\right)\right)\right) \stackrel{\delta}{\rightarrow} \pi_{-1}\left(f i b\left(\operatorname{cube}_{n+1}\left(\mathcal{F}^{q}\left(\Delta^{\bullet}\right)\right)\right)\right) \stackrel{(\eta \rho)_{*}}{\longrightarrow} \pi_{n-1}\left(\left|\mathcal{F}^{q}\left(\Delta^{\bullet}\right)\right|\right)
$$

to $z$.

We denote the simplicial prespectrum $\mathcal{K}^{\mathcal{C}^{q}}\left(\Delta^{\bullet}\right)$ by $X_{\bullet}$, the simplicial prespectrum $\left(n \mapsto \mathbb{B}\left(z^{q}(F, n)\right)\right)$ by $B_{\bullet}$, and the simplicial prespectrum $\mathcal{F}^{q}\left(\Delta^{\bullet}\right)$ by $\mathcal{F}_{\bullet}$.

To simplify matters slightly we make a few remarks.

(6.6.1). - The kernel of the homomorphism $j$ coincides with the kernel of the homomorphism

$$
\pi_{0}\left(f i b\left(c u b e_{n}\left(X_{\bullet}\right)\right)\right) \rightarrow \pi_{0}\left(f i b\left(\operatorname{cube}_{n}\left(\mathbb{B}\left(z^{q}(F, \bullet)\right)\right)\right)\right) .
$$

This follows immediately from (6.4.2) and the identification

$$
\pi_{0}\left(f i b\left(\left\{c u b e_{n}\left(X_{\bullet}\right)\right\}_{1}\right)\right)=\pi_{0}\left(f i b\left(\left\{\operatorname{cube}_{n}\left(B_{\bullet}\right)\right\}_{1}\right)\right) .
$$

(6.6.2). - The homomorphism $\pi_{0}\left(f i b\left(c u b e_{n}\left(\mathcal{F}_{\bullet}\right)\right) \rightarrow \pi_{0}\left(f i b\left(c u b e_{n}\left(X_{\bullet}\right)\right)\right.\right.$ is injective.

This follows immediately from (6.4.1) and the definition of $\mathcal{F}_{\bullet}=\mathcal{F}^{q}\left(\Delta^{\bullet}\right)$ (cf. (6.2.0)). 
Thus (6.6.0), refined by (6.6.1) and (6.6.2), leading to the computation of the image of $k^{\prime}([x])$ in $\pi_{n-1}\left(\mid \mathcal{F}^{q}\left(\Delta^{\bullet} \mid\right)\right.$, has the following form

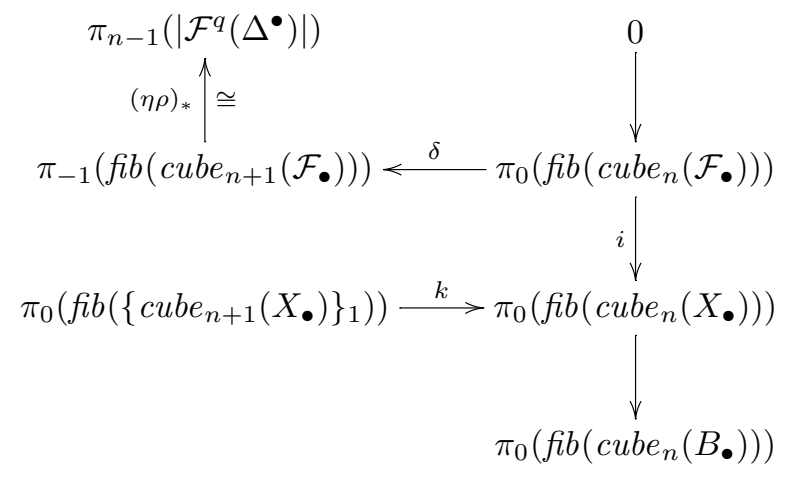

We consider the following commutative diagram (in which we dropped the notation fib everywhere so that it would fit the page), each of whose rows and columns are fibration sequences

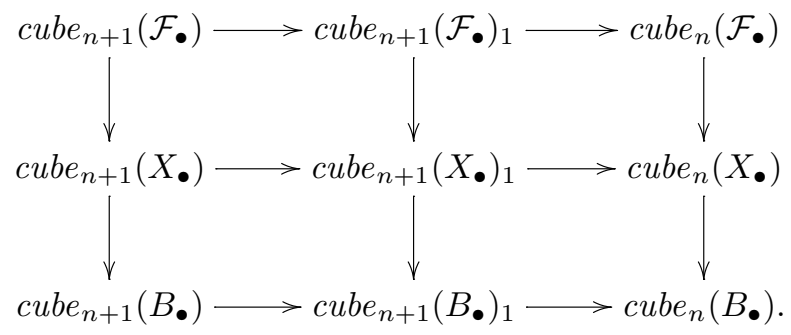

Note that $\pi_{1}\left(f i b\left(\operatorname{cube}_{n}\left(B_{\bullet}\right)\right)=0\right.$ according to (6.4.1) and hence the sequences obtained by applying the functor $\pi_{0}$ to both the rightmost column and the bottom row are left exact. We start with an element $x$ in the kernel of

$$
\pi_{0}\left(f i b\left(\operatorname{cube}_{n+1}\left(X_{\bullet}\right)_{1}\right)\right) \rightarrow \pi_{0}\left(f i b\left(\operatorname{cube}_{n}\left(B_{\bullet}\right)\right)\right)
$$

apply to it the diagram chase which may be described as "first go right, then up, then apply $\delta$ ". We can equally consider the diagram chase described as "first go down, then left, then apply $\delta$ ".

(6.6.3). - Both diagram chases give the same result (up to a sign).

Namely, the element $x$ comes from the (unique) element $x_{0} \in \pi_{0}\left(\mathcal{F}^{\prime}\right)$, where

$$
\mathcal{F}^{\prime}=f i b\left(f i b\left(\operatorname{cube}_{n+1}\left(X_{\bullet}\right)_{1}\right)\right) \rightarrow f i b\left(c u b e_{n}\left(B_{\bullet}\right)\right) .
$$

There are two canonical maps $p: \mathcal{F}^{\prime} \rightarrow f i b\left(\operatorname{cube}_{n+1}\left(B_{\bullet}\right)\right)$ and $q: \mathcal{F}^{\prime} \rightarrow f i b\left(\operatorname{cube}_{n}\left(\mathcal{F}_{\bullet}\right)\right)$ and the results of the two diagram chases are equal to $\delta q_{*}\left(x_{0}\right)$ and $\delta p_{*}\left(x_{0}\right)$ respectively. Our statement now follows from the usual relationship relating boundary maps in long exact sequences in homotopy groups associated to fibration sequences in (6.6.3). 
Furthermore, we have a commutative (up to a sign) diagram (see Proposition 4.6 and Lemma 4.7), in which we again omitted the notation $f i b$ in the top row

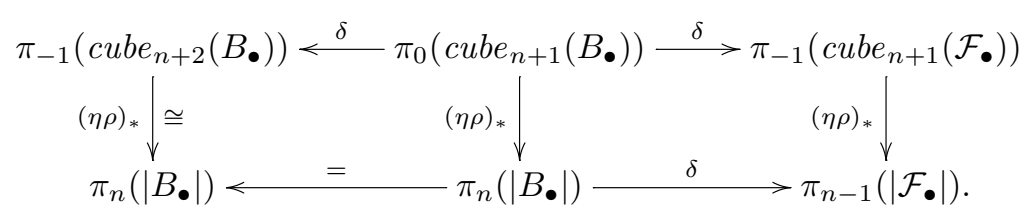

Finally, the diagram chase in which one goes down, then left, and then applies

$$
\pi_{0}\left(f i b\left(c u b e_{n+1}\left(B_{\bullet}\right)\right)\right) \stackrel{\delta}{\rightarrow} \pi_{-1}\left(f i b\left(c u b e_{n+2}\left(B_{\bullet}\right)\right)\right) \stackrel{\sim}{\rightarrow} \pi_{n}\left(\left|B_{\bullet}\right|\right)
$$

establishes the isomorphism $\left(j^{\prime}\right)^{-1}: E_{p, q}^{2} \stackrel{\sim}{\rightarrow} \pi_{n}\left(\left|B_{\bullet}\right|\right)$ used to identify these two groups.

\section{The homotopy invariance of the prespectrum $\mathcal{K}^{\mathcal{C}^{q}}\left(\Delta^{\bullet} \times X\right)$}

The main result of this section, Theorem 7.6, asserts for any smooth scheme $X$ over our fixed field $F$ that flat pull-back

$$
\mathcal{K}^{\mathcal{C}^{q}}\left(\Delta^{\bullet} \times X\right) \rightarrow \mathcal{K}^{\mathcal{C}^{q}}\left(\Delta^{\bullet} \times X \times \mathbb{A}^{1}\right)
$$

is a weak equivalence. As we see in Proposition 7.2 below, this "homotopy invariance" would be elementary if $\mathcal{K}^{\mathcal{C}^{q}}\left(\Delta^{\bullet} \times-\right)$ were a well-defined contravariant functor on smooth schemes. To overcome the lack of functoriality of $\mathcal{K}^{\mathcal{C}^{q}}\left(\Delta^{\bullet} \times-\right)$, we use a technique developed by Bloch by showing that suitable functoriality can be arranged on finite subcomplexes.

We start with the following elementary observation.

LEMMA 7.1. - Let $X \mapsto M(X)$ be a contravariant functor from the category $S m / F$ of smooth schemes of finite type over a field $F$ to the category of pointed spaces. For any $X \in S m / F$ the two continuous maps

$$
\left|M\left(\Delta^{\bullet} \times \mathbb{A}^{1} \times X\right)\right| \underset{i_{1}^{*}}{\stackrel{i_{0}^{*}}{\longrightarrow}}\left|M\left(\Delta^{\bullet} \times X\right)\right|
$$

induced by the embeddings $X \underset{i_{1}: x \mapsto 1 \times x}{\stackrel{i_{0}: x \mapsto 0 \times x}{\underset{i_{1}}{3}}} \mathbb{A}^{1} \times X$ are homotopic and hence induce the same homomorphisms in homotopy groups.

Proof. - Let $\mathrm{I}_{\bullet}$ denote the simplicial set corresponding to the poset $\{0<1\}$ (i.e., $n$-simplices of I. are non-decreasing sequences $\left.j_{0} \leqslant j_{1} \leqslant \cdots \leqslant j_{n}\left(j_{k} \in\{0<1\}\right)\right)$. We construct explicitly a simplicial homotopy

$$
\mathrm{I} \times\left|M\left(\Delta^{\bullet} \times \mathbb{A}^{1} \times X\right)\right|=\left|\mathrm{I}_{\bullet} \times M\left(\Delta^{\bullet} \times \mathbb{A}^{1} \times X\right)\right| \stackrel{H}{\rightarrow}\left|M\left(\Delta^{\bullet} \times X\right)\right|
$$

setting $\mathrm{H}_{n}(j \times m)=\left(f_{j} \times 1_{X}\right)^{*}(m)\left(j \in \mathrm{I}_{n}, m \in M\left(\Delta^{n} \times \mathbb{A}^{1} \times X\right)\right)$, where

$$
f_{j}: \Delta^{n} \rightarrow \Delta^{n} \times \mathbb{A}^{1}
$$

is the linear morphism sending the $k$ th vertex $v_{k} \in \Delta^{n}$ to $v_{k} \times j_{k} \in \Delta^{n} \times \mathbb{A}^{1}$. 
Remark 7.1.1. - The explicit form of the above homotopy shows immediately that its composition with the map $\left|M\left(\Delta^{\bullet} \times X\right)\right| \stackrel{p^{*}}{\longrightarrow}\left|M\left(\Delta^{\bullet} \times \mathbb{A}^{1} \times X\right)\right|$ (where $p: \mathbb{A}^{1} \times X \rightarrow X$ is the obvious projection) is the constant homotopy relating the identity endomorphism of $\left|M\left(\Delta^{\bullet} \times X\right)\right|$ to itself, in particular the above homotopy respects the distinguished points.

Lemma 7.1 easily implies the following proposition.

PROPOSITION 7.2. - Let $X \mapsto \mathcal{M}(X)$ be a contravariant functor from the category $\mathrm{Sm} / \mathrm{F}$ of smooth schemes of finite type over a field $F$ to the category of prespectra. Then for any $X \in S m / F$ the obvious morphism

$$
\begin{aligned}
\mathcal{M}\left(\Delta^{\bullet} \times X\right) & =\left|n \mapsto \mathcal{M}\left(\Delta^{n} \times X\right)\right| \stackrel{p^{*}}{\longrightarrow} \mathcal{M}\left(\Delta^{\bullet} \times \mathbb{A}^{1} \times X\right) \\
& =\left|n \mapsto \mathcal{M}\left(\Delta^{n} \times \mathbb{A}^{1} \times X\right)\right|
\end{aligned}
$$

induced by the projection $\mathbb{A}^{1} \times X \stackrel{p}{\rightarrow} X$ is a weak equivalence of prespectra.

Proof. - The embedding $i: X \stackrel{x \mapsto 0 \times x}{\longrightarrow} \mathbb{A}^{1} \times X$ is right inverse to $p$ and hence the induced morphism of prespectra $i^{*}$ is left inverse to $p^{*}$. The composition $i \circ p: \mathbb{A}^{1} \times X \rightarrow \mathbb{A}^{1} \times X$ is algebraically homotopic to the identity endomorphism, i.e., there exists a morphism

$$
H: \mathbb{A}^{1} \times\left(\mathbb{A}^{1} \times X\right) \stackrel{s \times t \times x \mapsto s t \times x}{\longrightarrow} \mathbb{A}^{1} \times X
$$

such that the restriction $H_{0}$ of $H$ to $0 \times\left(\mathbb{A}^{1} \times X\right)$ equals $i \circ p$, whereas the restriction $H_{1}$ of $H$ to $1 \times\left(\mathbb{A}^{1} \times X\right)$ equals the identity. Thus, Lemma 7.1 implies that the homomorphism in homotopy groups induced by $i \circ p$ equals the identity.

Proposition 7.2 does not apply to the prespectrum $\mathcal{K}^{\mathcal{C}^{q}}\left(\Delta_{X}^{\bullet}\right)$ since the assignment $X \mapsto \mathcal{K}^{\mathcal{C}^{q}}\left(\Delta_{X}^{\bullet}\right)$ is contravariant functorial only for flat morphisms. To prove the homotopy invariance of the prespectrum $\mathcal{K}^{\mathcal{C}^{q}}\left(\Delta_{X}^{\bullet}\right)$ we use a modification of a method developed by Bloch [3].

To simplify notation, we let $S_{\bullet}^{\left\{X_{i}\right\}}(X)$ denote the bisimplicial set

$$
S_{\bullet}^{\left\{X_{i}\right\}}(X) \equiv w S_{\bullet}\left(\mathcal{C P}^{\left\{X_{i}\right\}}(X)\right) .
$$

Here, $\mathcal{C P} \mathcal{P}^{\left\{X_{i}\right\}}(X)$ is the Waldhausen category of complexes of big vectors bundles on $X$ acyclic outside of the family of closed subschemes $\left\{X_{i} \subset X\right\}$ and $w S_{n}\left(\mathcal{C P}^{\left\{X_{i}\right.}(X)\right)$ is the subcategory of weak equivalences of the category on $n$-filtered objects of $\mathcal{S P}^{\left\{X_{i}\right\}}(X)$. The geometric realization of this simplicial set is the first term (i.e., the first delooping of the K-theory space) of the $\Omega$-prespectrum $\mathcal{K}^{\left\{X_{i}\right\}}(X)$, so that we can write

$$
\Omega^{-1} \mathcal{K}^{\left\{X_{i}\right\}}(X)=\left|S_{\bullet}^{\left\{X_{i}\right\}}(X)\right| .
$$

We extend this in the evident fashion to apply to cosimplicial varieties such as $\Delta^{\bullet} \times X$. We refer the reader to Appendix $\mathrm{C}$ for a brief discussion of these matters.

For any $a \in \mathbb{A}^{1}(F)$, we let

$$
\mathcal{C}_{a}^{q}\left(\Delta^{n} \times \mathbb{A}^{1} \times X\right)
$$

denote the family of supports consisting of all closed subschemes of $\Delta^{n} \times \mathbb{A}^{1} \times X$ which for any face $\Delta^{m} \subset \Delta^{n}$ intersect in codimension $\geqslant q$ the subschemes $\Delta^{m} \times \mathbb{A}^{1} \times X$ and $\Delta^{m} \times a \times X$. 
Since, according to the definition, the inverse image of any $Y \in \mathcal{C}_{a}^{q}\left(\Delta^{n} \times \mathbb{A}^{1} \times X\right)$ under the closed embedding

$$
i_{a}: \Delta^{n} \times X \stackrel{v \times x \mapsto v \times a \times x}{\longrightarrow} \Delta^{n} \times \mathbb{A}^{1} \times X
$$

is in $\mathcal{C}^{q}\left(\Delta^{n} \times X\right)$ we have canonical morphisms of spaces and prespectra (specialization at $a$ )

$$
s_{a}=i_{a}^{*}:\left|S_{\bullet}^{\mathcal{C}_{a}^{q}}\left(\Delta^{\bullet} \times \mathbb{A}^{1} \times X\right)\right| \rightarrow\left|S_{\bullet}^{\mathcal{C}^{q}}\left(\Delta^{\bullet} \times X\right)\right| .
$$

Assume now that we have two elements $a, b \in \mathbb{A}^{1}(F)$. Denote by $\mathcal{C}_{a, b}^{q}\left(\Delta^{n} \times \mathbb{A}^{1} \times X\right)$ the family of supports consisting of all closed subschemes $Z \in \mathcal{C}^{q}\left(\Delta^{n} \times \mathbb{A}^{1} \times X\right)$, whose inverse image under each of the morphisms

$$
\Delta^{n} \times X \stackrel{f_{j} \times 1_{X}}{\longrightarrow} \Delta^{n} \times \mathbb{A}^{1} \times X \stackrel{v \times \lambda \times x \mapsto v \times(1-\lambda) a+\lambda b \times x}{\longrightarrow} \Delta^{n} \times \mathbb{A}^{1} \times X
$$

is in $\mathcal{C}^{q}\left(\Delta^{n} \times X\right)$. Here $j=\left(j_{0} \leqslant j_{1} \leqslant \cdots \leqslant j_{n}\right), j_{k} \in\{0<1\}$ and $f_{j}$ is the morphism considered in the proof of Lemma 7.1; the second arrow is induced by the linear automorphism of $\mathbb{A}^{1}$, taking 0 to $a$ and 1 to $b$. Note that

$$
\mathcal{C}_{a, b}^{q}\left(\Delta^{n} \times \mathbb{A}^{1} \times X\right) \subset \mathcal{C}_{a}^{q}\left(\Delta^{n} \times \mathbb{A}^{1} \times X\right) \cap \mathcal{C}_{b}^{q}\left(\Delta^{n} \times \mathbb{A}^{1} \times X\right)
$$

and hence both specialization maps $s_{a}, s_{b}$ are defined on $\left|S_{\bullet}^{\mathcal{C}_{a, b}^{q}}\left(\Delta^{\bullet} \times \mathbb{A}^{1} \times X\right)\right|$. Using the same simplicial homotopy as in the proof of Lemma 7.1 we get immediately the following result.

LEMMA 7.3. - For any $a, b \in \mathbb{A}^{1}(F)$, the specialization maps of (7.2.4)

$$
\left|S_{\bullet}^{\mathcal{C}_{a, b}^{q}}\left(\Delta^{\bullet} \times \mathbb{A}^{1} \times X\right)\right| \underset{s_{b}}{\stackrel{s_{a}}{\longrightarrow}}\left|S_{\bullet}^{\mathcal{C}^{q}}\left(\Delta^{\bullet} \times X\right)\right|
$$

are homotopic.

The following lemma is easily proved by observing that $Y \in \mathcal{C}^{q}\left(\Delta^{n} \times \mathbb{A}^{1} \times X\right)$ is not in $\mathcal{C}_{a}^{q}\left(\Delta^{n} \times \mathbb{A}^{1} \times X\right)$ if and only if $Y$ satisfies at least one of a finite number of proper, closed conditions (of improper intersection with some $\Delta^{m} \times a \times X$ ). We leave the proof to the reader.

LEMma 7.4. - (1) Assume that $Y \in \mathcal{C}^{q}\left(\Delta^{n} \times \mathbb{A}^{1} \times X\right)$. Then $Y \in \mathcal{C}_{a}^{q}\left(\Delta^{n} \times \mathbb{A}^{1} \times X\right)$ for all but finitely many $a \in \mathbb{A}^{1}(F)$.

(2) Assume that $Y \in \mathcal{C}_{a}^{q}\left(\Delta^{n} \times \mathbb{A}^{1} \times X\right)$. Then $Y \in \mathcal{C}_{a, b}^{q}\left(\Delta^{n} \times \mathbb{A}^{1} \times X\right)$ for all but finitely many $b \in \mathbb{A}^{1}(F)$.

Lemmas 7.3 and 7.4 easily imply the following corollary.

COROllary 7.5. - Let $T \subset S_{\bullet}^{\mathcal{C}^{q}}\left(\Delta^{\bullet} \times \mathbb{A}^{1} \times X\right)$ be a simplicial subset with only finitely many non-degenerate simplices. Then $T \subset S_{\bullet}^{\mathcal{C}_{a}^{q}}\left(\Delta^{\bullet} \times \mathbb{A}^{1} \times X\right)$ for all but finitely many a, and hence for all but finitely many a we have a well defined specialization map

$$
s_{a}:|T| \rightarrow\left|S_{\bullet}^{\mathcal{C}^{q}}\left(\Delta^{\bullet} \times X\right)\right| .
$$

Moreover, if $F$ is infinite and if

$$
s_{b}:|T| \rightarrow\left|S_{\bullet}^{\mathcal{C}^{q}}\left(\Delta^{\bullet} \times X\right)\right|
$$

is another specialization map defined on $T$, then these two maps are homotopic. 
Proof. - The first statement is obvious from Lemma 7.4. To prove the second, observe that since $F$ is infinite there are elements $c \in \mathbb{A}^{1}(F)$ for which

$$
T \subset S_{\bullet}^{\mathcal{C}_{a, c}^{q}}\left(\Delta^{\bullet} \times \mathbb{A}^{1} \times X\right) \cap S_{\bullet}^{\mathcal{C}_{b, c}^{q}}\left(\Delta^{\bullet} \times \mathbb{A}^{1} \times X\right)
$$

and hence $s_{a \mid T} \simeq s_{c \mid T} \simeq s_{b \mid T}$ according to Lemma 7.3.

We can now prove the main result of this section.

THEOREM 7.6. - Assume that the field $F$ is infinite. For any $X \in S m / F$ and any $q \geqslant 0$, the canonical morphism of prespectra

$$
\mathcal{K}^{\mathcal{C}^{q}}\left(\Delta^{\bullet} \times X\right) \stackrel{p^{*}}{\rightarrow} \mathcal{K}^{\mathcal{C}^{q}}\left(\Delta^{\bullet} \times \mathbb{A}^{1} \times X\right)
$$

induced by the (flat) projection $\mathbb{A}^{1} \times X \stackrel{p}{\rightarrow} X$, is a weak equivalence, where $\mathcal{K}^{\mathcal{C}^{q}}(\Delta \bullet \times X)$ is the $\Omega$-spectrum of (5.0.2).

Proof. - We have to show that the induced homomorphisms of homotopy groups

$$
p^{*}: \pi_{i}\left(\left|S_{\bullet}^{\mathcal{C}^{q}}\left(\Delta^{\bullet} \times X\right)\right|\right) \rightarrow \pi_{i}\left(\left|S_{\bullet}^{\mathcal{C}^{q}}\left(\Delta^{\bullet} \times \mathbb{A}^{1} \times X\right)\right|\right)
$$

are isomorphisms. Corollary 7.5 shows that we have well-defined specialization homomorphisms on homotopy groups

$$
s=s^{X}: \pi_{i}\left(\left|S_{\bullet}^{\mathcal{C}^{q}}\left(\Delta^{\bullet} \times \mathbb{A}^{1} \times X\right)\right|\right) \rightarrow \pi_{i}\left(\left|S_{\bullet}^{\mathcal{C}^{q}}\left(\Delta^{\bullet} \times X\right)\right|\right),
$$

and the composition

$$
\pi_{i}\left(\left|S_{\bullet}^{\mathcal{C}^{q}}\left(\Delta^{\bullet} \times X\right)\right|\right) \stackrel{p^{*}}{\rightarrow} \pi_{i}\left(\left|S_{\bullet}^{\mathcal{C}^{q}}\left(\Delta^{\bullet} \times \mathbb{A}^{1} \times X\right)\right|\right) \stackrel{s}{\rightarrow} \pi_{i}\left(\left|S_{\bullet}^{\mathcal{C}^{q}}\left(\Delta^{\bullet} \times X\right)\right|\right)
$$

is obviously the identity. To show that the other composition is also the identity it suffices to consider elements coming from $\pi_{i}$ of an appropriate $\left|S_{\bullet}^{\mathcal{C}_{a}^{q}}\left(\Delta^{\bullet} \times \mathbb{A}^{1} \times X\right)\right|$. Making if necessary a translation we may even restrict our attention to elements coming from $\pi_{i}(|T|)$, where $T \subset S_{\bullet}^{\mathcal{C}_{0}^{q}}\left(\Delta^{\bullet} \times \mathbb{A}^{1} \times X\right)$ is a finitely generated simplicial subset. Set $Y=\mathbb{A}^{1} \times X$ and consider the flat morphism

$$
m: \mathbb{A}^{1} \times Y=\mathbb{A}^{1} \times \mathbb{A}^{1} \times X \stackrel{a \times b \times x \mapsto a b \times x}{\longrightarrow} Y=\mathbb{A}^{1} \times X .
$$

A straightforward verification shows that the exact functor $m^{*}$ takes $S_{\bullet}^{\mathcal{C}^{q}}\left(\Delta^{\bullet} \times \mathbb{A}^{1} \times X\right)$ to $S_{\bullet}^{\mathcal{C}_{1}^{q}}\left(\Delta^{\bullet} \times \mathbb{A}^{1} \times Y\right)$ and takes $S_{\bullet}^{\mathcal{C}_{0}^{q}}\left(\Delta^{\bullet} \times \mathbb{A}^{1} \times X\right)$ to $S_{\bullet}^{\mathcal{C}_{0}^{q}}\left(\Delta^{\bullet} \times \mathbb{A}^{1} \times Y\right)$. Moreover the following diagrams of spaces commute

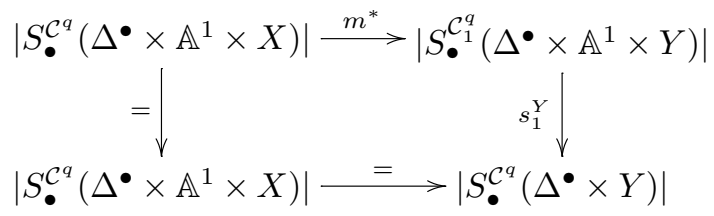

$4^{\mathrm{e}}$ SÉRIE - TOME $35-2002-\mathrm{N}^{\circ} 6$ 


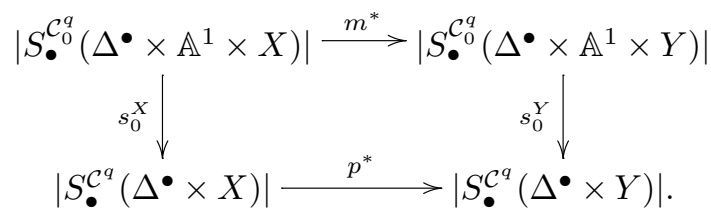

Applying Corollary 7.5 to $Y$ and using the above commutative diagrams we conclude that the restriction of $p^{*} \circ s_{0}^{X}$ to $|T|$ is homotopic to the identity map which concludes the proof.

Theorem 7.6 implies in particular the following extension of Theorem 6.2.

COROLLARY 7.7. - For any $n, q \geqslant 0$ the following sequence of $\Omega$-prespectra is a homotopy fibration sequence

$$
\mathcal{K}^{\mathcal{C}^{q+1}}\left(\Delta^{\bullet} \times \mathbb{A}^{n}\right) \rightarrow \mathcal{K}^{\mathcal{C}^{q}}\left(\Delta^{\bullet} \times \mathbb{A}^{n}\right) \rightarrow \mathbb{B}\left(z^{q}\left(\mathbb{A}^{n}, \bullet\right)\right)
$$

More precisely the composition of the above maps is trivial and the induced map

$$
\mathcal{K}^{\mathcal{C}^{q+1}}\left(\Delta^{\bullet} \times \mathbb{A}^{n}\right) \rightarrow f i b\left(\mathcal{K}^{\mathcal{C}^{q}}\left(\Delta^{\bullet} \times \mathbb{A}^{n}\right) \rightarrow \mathbb{B}\left(z^{q}\left(\mathbb{A}^{n}, \bullet\right)\right)\right)
$$

is a weak equivalence.

Proof. - Observe that the projection $p: \mathbb{A}^{n} \rightarrow \operatorname{Spec} F$ gives us a commutative diagram all vertical arrows of which are weak equivalences,

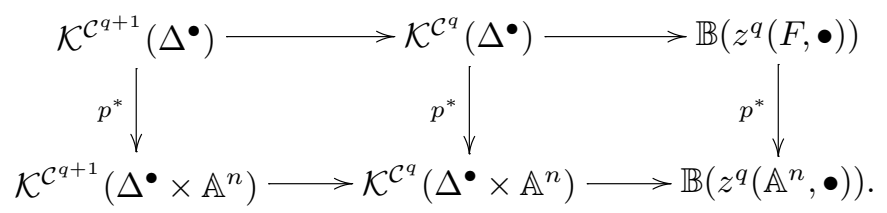

Since the top row is a homotopy fibration sequence by Theorem 6.2, the bottom row is a homotopy fibration sequence as well.

We conclude this section with few further remarks concerning the situation discussed above. For any $a \in \mathbb{A}^{1}(F)$ denote by $l_{a}: \mathbb{A}^{1} \stackrel{t \mapsto t+a}{\longrightarrow} \mathbb{A}^{1}$ the automorphism "translation by $a$ ".

LEMMA 7.8. - The associated map of spaces

$$
l_{a}^{*}:\left|S_{\bullet}^{\mathcal{C}^{q}}\left(\Delta^{\bullet} \times \mathbb{A}^{1} \times X\right)\right| \rightarrow\left|S_{\bullet}^{\mathcal{C}^{q}}\left(\Delta^{\bullet} \times \mathbb{A}^{1} \times X\right)\right|
$$

is homotopic to the identity map.

Proof. - Set $Y=\mathbb{A}^{1} \times X$ and denote by $l: \mathbb{A}^{1} \times Y=\mathbb{A}^{1} \times \mathbb{A}^{1} \times X \rightarrow \mathbb{A}^{1} \times X$ the morphism taking $a \times b \times x$ to $(a+b) \times x$. A straightforward verification shows that $l^{*}$ takes $\left|S_{\bullet}^{\mathcal{C}^{q}}\left(\Delta^{\bullet} \times \mathbb{A}^{1} \times X\right)\right|$ to $\left|S_{\bullet}^{\mathcal{C}_{0, a}^{q}}\left(\Delta^{\bullet} \times \mathbb{A}^{1} \times Y\right)\right|$. Since $s_{0}^{Y} l^{*}=i d, s_{a}^{Y} l^{*}=l_{a}^{*}$ our statement follows from Lemma 7.3.

One derives easily from the above proof an explicit simplicial homotopy relating $l_{a}^{*}$ and the identity map.

$$
\mathrm{H}_{n}(j \times m)=\left(f_{j} \times 1_{X}\right)^{*}(m) \quad\left(j \in \mathrm{I}_{n}, m \in\left|S_{\bullet}^{\mathcal{C}^{q}}\left(\Delta^{n} \times \mathbb{A}^{1} \times X\right)\right|\right)
$$


where this time $f_{j}: \Delta^{n} \times \mathbb{A}^{1} \rightarrow \Delta^{n} \times \mathbb{A}^{1}$ is an automorphism given by the formula

$$
\left(t_{0}, \ldots, t_{n}\right) \times t \mapsto\left(t_{0}, \ldots, t_{n}\right) \times\left(t_{0} j_{0}+\cdots+t_{n} j_{n}\right) a+t .
$$

The same simplicial homotopy may be used to prove the following result.

LEMMA 7.9. - The map

$$
l_{a}^{*}:\left|S_{\bullet}^{\mathcal{C}_{0, a}^{q}}\left(\Delta^{\bullet} \times \mathbb{A}^{1} \times X\right)\right| \rightarrow\left|S_{\bullet}^{\mathcal{C}_{0}^{q}}\left(\Delta^{\bullet} \times \mathbb{A}^{1} \times X\right)\right|
$$

is homotopic to the natural embedding.

Applying Corollary 7.5 and Lemma 7.9, we conclude the following.

COROLlary 7.10.- Assume that the field $F$ is infinite. Then the natural embedding $\left|S_{\bullet}^{\mathcal{C}^{q}}\left(\Delta^{\bullet} \times \mathbb{A}^{1} \times X\right)\right| \hookrightarrow\left|S_{\bullet}^{\mathcal{C}^{q}}\left(\Delta^{\bullet} \times \mathbb{A}^{1} \times X\right)\right|$ is a weak equivalence.

Proof. - For any finitely generated simplicial subset $T \subset S_{\bullet}^{\mathcal{C}^{q}}\left(\Delta^{\bullet} \times \mathbb{A}^{1} \times X\right)$ one can find $a \in \mathbb{A}^{1}(F)$ such that $T \subset S_{\bullet}^{\mathcal{C}^{q}}\left(\Delta^{\bullet} \times \mathbb{A}^{1} \times X\right)$ and consider the composition

$$
|T| \hookrightarrow\left|S_{\bullet}^{\mathcal{C}_{a}^{q}}\left(\Delta^{\bullet} \times \mathbb{A}^{1} \times X\right)\right| \stackrel{l_{a}^{*}}{\longrightarrow}\left|S_{\bullet}^{\mathcal{C}_{0}^{q}}\left(\Delta^{\bullet} \times \mathbb{A}^{1} \times X\right)\right| .
$$

Lemma 7.9 implies that this map is (up to homotopy) independent of the choice of $a$ (cf. the proof of Corollary 7.5) and that the composition

$$
|T| \rightarrow\left|S_{\bullet}^{\mathcal{C}_{0}^{q}}\left(\Delta^{\bullet} \times \mathbb{A}^{1} \times X\right)\right| \hookrightarrow\left|S_{\bullet}^{\mathcal{C}^{q}}\left(\Delta^{\bullet} \times \mathbb{A}^{1} \times X\right)\right|
$$

is homotopic to the geometric realization of the given simplicial inclusion. In this way we get canonical homomorphisms $\pi_{i}\left(\left|S_{\bullet}^{\mathcal{C}^{q}}\left(\Delta^{\bullet} \times \mathbb{A}^{1} \times X\right)\right|\right) \rightarrow \pi_{i}\left(\left|S_{\bullet}^{\mathcal{C}_{0}^{q}}\left(\Delta^{\bullet} \times \mathbb{A}^{1} \times X\right)\right|\right)$ inverse to the homomorphisms induced by the embedding $\left|S_{\bullet}^{\mathcal{C}_{0}^{q}}\left(\Delta^{\bullet} \times \mathbb{A}^{1} \times X\right)\right| \hookrightarrow\left|S_{\bullet}^{\mathcal{C}^{q}}\left(\Delta^{\bullet} \times \mathbb{A}^{1} \times X\right)\right|$.

Remark 7.11. - We will show below (in Theorem 9.6) how to eliminate the assumption that the field $F$ be infinite in the results of this and the next section.

\section{The $\Omega$-prespectrum $\mathcal{K}^{\mathcal{C}^{q}}\left(\Delta^{\bullet} \times \mathbb{A}^{q}\right)$ and sheaves with quasifinite support}

For schemes $X, S \in S m / F$, we denote by $\mathcal{K}^{\mathcal{Q}, S}(X)$ the $K$-theory prespectrum of the scheme $X \times S$ with family of supports $\mathcal{Q}(X \times S)$ consisting of all closed subschemes quasi-finite over $X$,

$$
\mathcal{K}^{\mathcal{Q}, S}(X) \equiv \mathcal{K}^{\mathcal{Q}(X \times S)}(X \times S) .
$$

The purpose of this section is to demonstrate that the $\Omega$-prespectra $\mathcal{K}^{\mathcal{C}^{q}}\left(\Delta^{\bullet}\right)$ can be replaced up to weak equivalence by the $\Omega$-prespectra $\mathcal{K}^{\mathcal{Q}, \mathbb{A}^{q}}\left(\Delta^{\bullet}\right)$. A major advantage of such a replacement is that $X \mapsto \mathcal{K}^{\mathcal{Q}, \mathbb{A}^{q}}\left(\Delta^{\bullet} \times X\right)$ is a well defined contravariant functor on the category $\mathrm{Sm} / \mathrm{F}$ by the discussion of Appendix $C$.

The technique we employ is borrowed from [29] where it is shown that if Bloch's condition on codimension $q$ cycles on $\Delta^{n} \times X$ that the cycles have good intersection with all faces is replaced (for $q$ less than or equal to the dimension of $X$ ) by the stronger condition that the cycles be equidimensional over $\Delta^{n}$ then the resulting complex is weakly equivalent to Bloch's complex $z^{q}(X, \bullet)$.

Throughout this section the field $F$ is assumed to be infinite.

We begin by recalling the key technical "moving" result which permits such a replacement. 
THEOREM 8.1 [29]. - Let $S$ be an affine scheme, let further $V$ be a closed subscheme in $\Delta^{n} \times S$ and let $t$ be a non-negative integer with the property $\operatorname{dim} V \leqslant n+t$. Assume that we are given an effective divisor $Z \subset \Delta^{n}$ and an $S$-morphism $\psi: Z \times S \rightarrow \Delta^{n} \times S$. Then there exists an $S$-morphism $\phi: \Delta^{n} \times S \rightarrow \Delta^{n} \times S$ such that

(1) $\left.\phi\right|_{Z \times S}=\psi$.

(2) The fibers of the projection $\phi^{-1}(V) \rightarrow \Delta^{n}$ over points of $\Delta^{n} \backslash Z$ are of dimension $\leqslant t$.

Let $\phi_{\bullet}: \Delta^{\bullet} \times \mathbb{A}^{q} \rightarrow \Delta^{\bullet} \times \mathbb{A}^{q}$ be a family of $\mathbb{A}^{q}$-morphisms such that for every strictly increasing map $\theta:[m] \rightarrow[n]$ the diagram

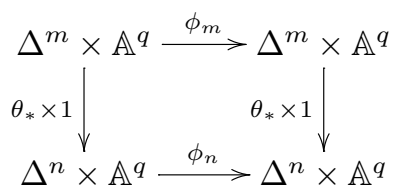

commutes. In this situation we will say that $\phi_{\bullet}$ is a pseudo endomorphism of the cosimplicial scheme $\Delta^{\bullet} \times \mathbb{A}^{q}$ (over $\left.\mathbb{A}^{q}\right)$. Denote by ${ }_{\phi} \mathcal{C}^{q}$ respectively ${ }_{\phi} \mathcal{Q}$ the family of supports on $\Delta^{n} \times \mathbb{A}^{q}$ consisting of those $Y \in \mathcal{C}^{q}\left(\Delta^{n} \times \mathbb{A}^{q}\right)$ (respectively $Y \in \mathcal{Q}\left(\Delta^{n} \times \mathbb{A}^{q}\right)$ ) whose inverse image under $\phi_{n}$ still belongs to $\mathcal{C}^{q}\left(\Delta^{n} \times \mathbb{A}^{p}\right)$ (respectively to $\mathcal{Q}\left(\Delta^{n} \times \mathbb{A}^{q}\right)$ ). For each $n$, the morphism $\phi_{n}$ defines a map

$$
\phi_{n}^{*}:\left|S_{\bullet}^{\phi^{\mathcal{C}^{q}}}\left(\Delta^{n} \times \mathbb{A}^{q}\right)\right| \rightarrow\left|S_{\bullet}^{\mathcal{C}^{q}}\left(\Delta^{n} \times \mathbb{A}^{q}\right)\right| .
$$

These maps are compatible with the maps induced by the strictly increasing $\theta:[m] \rightarrow[n]$ and hence give a map on Segal realizations

$$
\left\|n \mapsto\left|S_{\bullet}^{\mathcal{C}^{q}}\left(\Delta^{n} \times \mathbb{A}^{q}\right)\right|\right\| \stackrel{\phi^{*}}{\longrightarrow}\left\|n \mapsto\left|S_{\bullet}^{\mathcal{C}^{q}}\left(\Delta^{n} \times \mathbb{A}^{q}\right)\right|\right\|
$$

In the same way we get a map

$$
\left\|n \mapsto\left|S_{\bullet}^{\phi^{\mathcal{Q}}}\left(\Delta^{n} \times \mathbb{A}^{q}\right)\right|\right\| \stackrel{\phi^{*}}{\longrightarrow}\left\|n \mapsto\left|S_{\bullet}^{\mathcal{Q}}\left(\Delta^{n} \times \mathbb{A}^{q}\right)\right|\right\| .
$$

Using Theorem 8.1, we establish the existence of pseudo endomorphisms transporting a finite family $\left\{Y_{i}^{n}\right\} \subset \mathcal{C}^{q}\left(\Delta^{n} \times \mathbb{A}^{q}\right)$ to a family of subschemes quasi-finite over $\Delta^{n}$.

Proposition 8.2. - Assume that we are given an integer $N \geqslant 0$ and for each $0 \leqslant n \leqslant N$ a finite subfamily $\left\{Y_{i}^{n}\right\} \subset \mathcal{C}^{q}\left(\Delta^{n} \times \mathbb{A}^{q}\right)$. Then there exists a pseudo endomorphism $\phi_{\bullet}: \Delta^{\bullet} \times \mathbb{A}^{q} \rightarrow \Delta^{\bullet} \times \mathbb{A}^{q}$ such that

$$
\phi_{n}^{-1}\left(Y_{i}^{n}\right) \in \mathcal{Q}\left(\Delta^{n} \times \mathbb{A}^{q}\right) \quad \forall 0 \leqslant n \leqslant N, \forall i
$$

and, in particular, $Y_{i}^{n} \in{ }_{\phi} \mathcal{C}^{q}\left(\Delta^{n} \times \mathbb{A}^{q}\right)$.

Proof. - We may assume obviously that for any strictly increasing map $\theta:[m] \rightarrow[n]$ $(0 \leqslant m \leqslant n \leqslant N)$ and for any $i$ the scheme $\left(\theta_{*} \times 1_{\mathbb{A}^{q}}\right)^{-1}\left(Y_{i}^{n}\right)$ is a member of the family $\left\{Y_{j}^{m}\right\}$. We proceed to construct $\phi_{n}: \Delta^{n} \times \mathbb{A}^{q} \rightarrow \Delta^{n} \times \mathbb{A}^{q}$ which satisfy the following properties:

(1) The following diagrams commute (in which $\delta_{i}:[n-1] \rightarrow[n](0 \leqslant i \leqslant n)$ is the strictly increasing map missing $i$ ) 


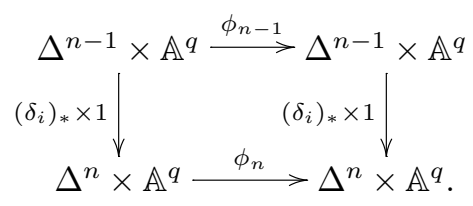

(2) If $n \leqslant N$ then $\phi_{n}^{-1}\left(Y_{i}^{n}\right) \in \mathcal{Q}\left(\Delta^{n} \times \mathbb{A}^{q}\right) \forall i$.

Assume that we have already constructed $\phi_{0}, \ldots, \phi_{n-1}$ which satisfy the above properties. Commutativity of diagrams (8.2.0) determines the morphism $\phi_{n}$ on each of the faces $\Delta_{i}^{n} \times \mathbb{A}^{q} \subset \Delta^{n} \times \mathbb{A}^{q}\left(\Delta_{i}^{n} \subset \Delta^{n}\right.$ is the divisor given by the equation $\left.t_{i}=0\right)$. Moreover the inductive assumption implies that these data are compatible one with another and define a morphism

$$
\psi: \partial \Delta^{n} \times \mathbb{A}^{q} \rightarrow \Delta^{n} \times \mathbb{A}^{q},
$$

where $\partial \Delta^{n} \subset \Delta^{n}$ is the divisor given by the equation $t_{0} \cdots t_{n}=0$. According to Theorem 8.1 we may extend $\psi$ to a morphism $\phi_{n}: \Delta^{n} \times \mathbb{A}^{q} \rightarrow \Delta^{n} \times \mathbb{A}^{q}$, so that the projection $\phi_{n}^{-1}\left(Y_{i}^{n}\right) \rightarrow \Delta^{n}$ is quasifinite outside $\partial \Delta^{n}$. However, over $\partial \Delta^{n}$ the above projection is quasifinite according to the induction assumption. Thus $\phi_{n}^{-1}\left(Y_{i}^{n}\right)$ is quasifinite over $\Delta^{n}$.

The following is an immediate corollary of Proposition 8.2.

Corollary 8.3. - For any compact subset $K \subset\left\|n \mapsto\left|S_{\bullet}^{\mathcal{C}^{q}}\left(\Delta^{n} \times \mathbb{A}^{q}\right)\right|\right\|$, there exists a pseudo endomorphism $\phi_{\bullet}$ such that

$$
K \subset\left\|n \mapsto\left|S_{\bullet}^{{ }^{\mathcal{C}^{q}}}\left(\Delta^{n} \times \mathbb{A}^{p}\right)\right|\right\| \quad \text { and } \quad \phi^{*}(K) \subset\left\|n \mapsto\left|S_{\bullet}^{\mathcal{Q}}\left(\Delta^{n} \times \mathbb{A}^{q}\right)\right|\right\| .
$$

Our next objective is to show that the embeddings of $K$ and $\phi^{*}(K)$ in Corollary 8.3 above are homotopic. To do so, we repeat the argument of Proposition 8.2 to construct a homotopy.

By a homotopy between $\phi_{\bullet}$ and the identity endomorphism we mean a pseudo endomorphism

$$
\Phi_{\bullet}: \Delta^{\bullet} \times \mathbb{A}^{1} \times \mathbb{A}^{q} \rightarrow \Delta^{\bullet} \times \mathbb{A}^{1} \times \mathbb{A}^{q}
$$

of the cosimplicial scheme $\Delta^{\bullet} \times \mathbb{A}^{1} \times \mathbb{A}^{q}$ (over $\mathbb{A}^{q}$ ) such that the following diagrams commute (in which $i_{0}$ and $i_{1}$ denote closed embeddings defined by points $0,1 \in \mathbb{A}^{1}$ )

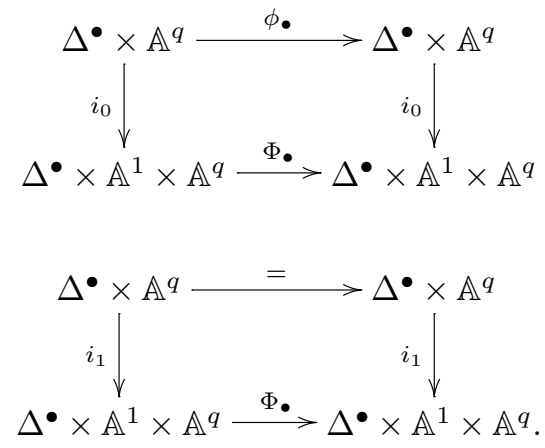

For a homotopy $\Phi$. as above let ${ }_{\Phi} \mathcal{C}^{q}\left(\Delta^{n} \times \mathbb{A}^{1} \times \mathbb{A}^{q}\right)$ (respectively ${ }_{\Phi} \mathcal{Q}\left(\Delta^{n} \times \mathbb{A}^{1} \times \mathbb{A}^{q}\right)$ ) be a family of supports on $\Delta^{n} \times \mathbb{A}^{1} \times \mathbb{A}^{q}$, consisting of those closed subschemes $Y \in \mathcal{C}^{q}\left(\Delta^{n} \times \mathbb{A}^{1} \times \mathbb{A}^{q}\right)$ (respectively, $\left.Y \in \mathcal{Q}\left(\left(\Delta^{n} \times \mathbb{A}^{1}\right) \times \mathbb{A}^{q}\right)\right)$ whose inverse image under any morphism of the form

$$
\Phi_{n} \circ\left(f_{j} \times 1_{\mathbb{A}^{q}}\right): \Delta^{n} \times \mathbb{A}^{q} \rightarrow \Delta^{n} \times \mathbb{A}^{1} \times \mathbb{A}^{q}
$$


belongs to $\mathcal{C}^{q}\left(\Delta^{n} \times \mathbb{A}^{q}\right)$ (respectively to $\mathcal{Q}\left(\Delta^{n} \times \mathbb{A}^{q}\right)$ ). Here, $j=\left(j_{0} \leqslant j_{1} \leqslant \cdots \leqslant j_{n}\right) j_{i} \in$ $\{0<1\}$ is a non-decreasing sequence and $f_{j}$ is a morphism defined in the proof of Lemma 7.1).

Let ${ }_{\Phi} \mathcal{C}^{q}\left(\Delta^{n} \times \mathbb{A}^{q}\right)$ (respectively ${ }_{\Phi} \mathcal{Q}\left(\Delta^{n} \times \mathbb{A}^{q}\right)$ ) denote the family of supports on $\Delta^{n} \times \mathbb{A}^{q}$, consisting of those closed subschemes $Y$, whose inverse image under the projection

$$
p: \Delta^{n} \times \mathbb{A}^{1} \times \mathbb{A}^{q} \rightarrow \Delta^{n} \times \mathbb{A}^{q}
$$

is in ${ }_{\Phi} \mathcal{C}^{q}\left(\Delta^{n} \times \mathbb{A}^{1} \times \mathbb{A}^{q}\right)$ (respectively is in ${ }_{\Phi} \mathcal{Q}\left(\Delta^{n} \times \mathbb{A}^{1} \times \mathbb{A}^{q}\right)$ ).

Proposition 8.4. - Let $\phi_{\bullet}: \Delta^{\bullet} \times \mathbb{A}^{q} \rightarrow \Delta^{\bullet} \times \mathbb{A}^{q}$ be a pseudo endomorphism of $\Delta^{\bullet} \times \mathbb{A}^{q}$. Assume that we are given an integer $N \geqslant 0$ and for each $0 \leqslant n \leqslant N$ a finite subfamily $\left\{Y_{i}^{n}\right\} \subset{ }_{\phi} \mathcal{C}^{q}\left(\Delta^{n} \times \mathbb{A}^{q}\right)$ (respectively $\left.\left\{Y_{i}^{n}\right\} \subset{ }_{\phi} \mathcal{Q}\left(\Delta^{n} \times \mathbb{A}^{q}\right)\right)$. Then there exists a homotopy

$$
\Phi_{\bullet}: \Delta^{\bullet} \times \mathbb{A}^{1} \times \mathbb{A}^{q} \rightarrow \Delta^{\bullet} \times \mathbb{A}^{1} \times \mathbb{A}^{q}
$$

between $\phi_{\cdot}$ and the identity endomorphism such that $Y_{i}^{n} \in{ }_{\Phi} \mathcal{C}^{q}\left(\Delta^{n} \times \mathbb{A}^{q}\right)$ (respectively $\left.Y_{i}^{n} \in{ }_{\Phi} \mathcal{Q}\left(\Delta^{n} \times \mathbb{A}^{p}\right)\right)$.

Proof. - We may assume obviously that for any strictly increasing map $\theta:[m] \rightarrow[n]$ $(0 \leqslant m \leqslant n \leqslant N)$ and for any $i$ the scheme $\left(\theta_{*} \times 1_{\mathbb{A}^{q}}\right)^{-1}\left(Y_{i}^{n}\right)$ is a member of the family $\left\{Y_{j}^{m}\right\}$. We proceed to construct

$$
\Phi_{n}: \Delta^{n} \times \mathbb{A}^{1} \times \mathbb{A}^{q} \rightarrow \Delta^{n} \times \mathbb{A}^{1} \times \mathbb{A}^{q}
$$

which satisfy the following properties

(1) The following diagrams (in which $\delta_{i}:[n-1] \rightarrow[n](0 \leqslant i \leqslant n)$ is the strictly increasing map missing $i$ ) commute

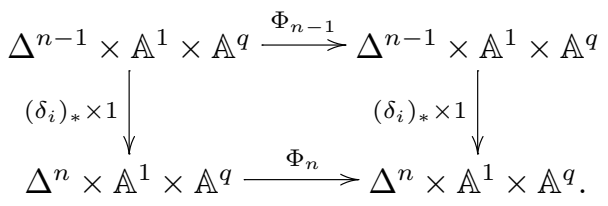

(2) The following diagrams commute

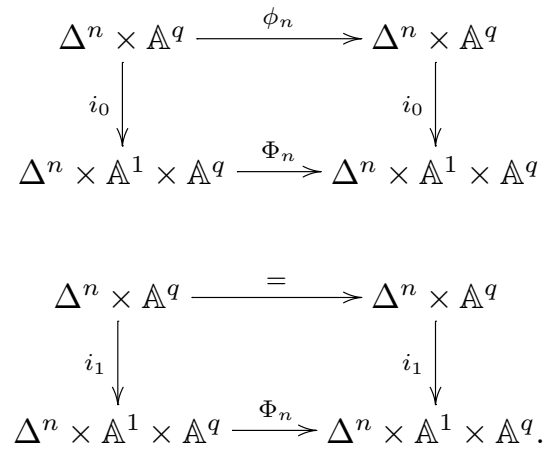

(3) If $n \leqslant N$ then for any $i$ the projection $\Phi_{n}^{-1}\left(Y_{i}^{n} \times \mathbb{A}^{1}\right) \rightarrow \Delta^{n} \times \mathbb{A}^{1}$ is quasifinite over $\Delta^{n} \times\left(\mathbb{A}^{1} \backslash\{0,1\}\right)$. 
The construction of $\Phi$ 's repeats essentially verbatim (with $\partial \Delta^{n} \times \mathbb{A}^{q}$ replaced by $\left(\partial \Delta^{n} \times\right.$ $\left.\left.\mathbb{A}^{1} \times \mathbb{A}^{q}\right) \cup\left(\Delta^{n} \times\{0,1\} \times \mathbb{A}^{q}\right)\right)$ the construction of $\phi$ 's in the proof of Proposition 8.2 and we skip the obvious details.

In the case of the family of supports $\mathcal{Q}$, we can say moreover that the projection

$$
\Phi_{n}^{-1}\left(Y_{i}^{n} \times \mathbb{A}^{1}\right) \rightarrow \Delta^{n} \times \mathbb{A}^{1}
$$

is quasifinite over all $\Delta^{n} \times \mathbb{A}^{1}$ (over $\Delta^{n} \times\{0,1\}$ it is quasifinite since $Y_{i}^{n} \in{ }_{\phi} \mathcal{Q}\left(\Delta^{n} \times \mathbb{A}^{p}\right)$ ). This implies readily that the inverse image of $\Phi_{n}^{-1}\left(Y_{i}^{n} \times \mathbb{A}^{1}\right)$ under the morphism

$$
f_{j} \times 1: \Delta^{n} \times \mathbb{A}^{q} \rightarrow \Delta^{n} \times \mathbb{A}^{1} \times \mathbb{A}^{q}
$$

is quasifinite over $\Delta^{n}$, i.e., $Y_{i}^{n} \in{ }_{\Phi} \mathcal{Q}\left(\Delta^{n} \times \mathbb{A}^{q}\right)$, which finishes the proof in this case.

In the case of the family of supports $\mathcal{C}^{q}$, one readily verifies that the inverse image of $\Phi_{n}^{-1}\left(Y_{i}^{n} \times \mathbb{A}^{1}\right)$ under the morphism $f_{j} \times 1: \Delta^{n} \times \mathbb{A}^{q} \rightarrow \Delta^{n} \times \mathbb{A}^{1} \times \mathbb{A}^{q}$ is of codimension $\geqslant q$. Since the pull back of this scheme under the morphism

$$
\Delta^{m} \times \mathbb{A}^{q} \stackrel{\theta_{*} \times 1}{\longrightarrow} \Delta^{n} \times \mathbb{A}^{q}
$$

corresponding to the strictly increasing map $\theta:[m] \rightarrow[n]$ may be obtained in a similar way from $\left(\left(\theta_{*} \times 1_{\mathbb{A}^{q}}\right)^{-1} Y_{i}^{n}\right) \times \mathbb{A}^{1}$ we conclude that

$$
\left(\Phi_{n} \circ\left(f_{j} \times 1\right)\right)^{-1}\left(Y_{i}^{n}\right) \in \mathcal{C}^{q}\left(\Delta^{n} \times \mathbb{A}^{q}\right)
$$

i.e., $Y_{i}^{n} \in{ }_{\Phi} \mathcal{C}^{q}\left(\Delta^{n} \times \mathbb{A}^{q}\right)$.

Two maps between pointed spaces

$$
\left(X, x_{0}\right) \underset{g}{\stackrel{f}{\longrightarrow}}\left(Y, y_{0}\right)
$$

are weakly homotopic if their restrictions to any compact subspace $K \subset X$ containing $x_{0}$ are related by a (base point preserving) homotopy. Proposition 8.4 leads to the following important result about pseudo endomorphisms.

PROPOSITION 8.5. - Let $\phi \bullet$ be a pseudo endomorphism of the cosimplicial scheme $\Delta^{\bullet} \times \mathbb{A}^{q}$. Then the morphisms

$$
\begin{aligned}
& \left\|n \mapsto\left|S_{\bullet}^{\phi^{\mathcal{C}}}\left(\Delta^{n} \times \mathbb{A}^{q}\right)\right|\right\| \stackrel{\phi^{*}}{\longrightarrow}\left\|n \mapsto\left|S_{\bullet}^{\mathcal{C}^{q}}\left(\Delta^{n} \times \mathbb{A}^{q}\right)\right|\right\|, \\
& \left\|n \mapsto\left|S_{\bullet}^{\mathcal{Q}}\left(\Delta^{n} \times \mathbb{A}^{q}\right)\right|\right\| \stackrel{\phi^{*}}{\longrightarrow}\left\|n \mapsto\left|S_{\bullet}^{\mathcal{Q}}\left(\Delta^{n} \times \mathbb{A}^{q}\right)\right|\right\|,
\end{aligned}
$$

are weakly homotopic to the canonical inclusion maps.

Proof. - Let $\Phi_{\bullet}$ be a homotopy between $\phi_{\bullet}$ and the identity endomorphism. Associating to every pair $j \times m \in \mathrm{I}_{n} \times\left|S_{\bullet}^{\Phi} \mathcal{C}^{q}\left(\Delta^{n} \times \mathbb{A}^{1} \times \mathbb{A}^{q}\right)\right|$ the element

$$
\left(\Phi_{n} \circ\left(f_{j} \times 1_{\mathbb{A}^{q}}\right)\right)^{*}(m) \in\left|S_{\bullet}^{\mathcal{C}^{q}}\left(\Delta^{n} \times \mathbb{A}^{p}\right)\right|,
$$

we get a sequence of maps

$$
\mathrm{H}_{n}: \mathrm{I}_{n} \times\left|S_{\bullet}^{\mathcal{C}^{q}}\left(\Delta^{n} \times \mathbb{A}^{1} \times \mathbb{A}^{q}\right)\right| \rightarrow\left|S_{\bullet}^{\mathcal{C}^{q}}\left(\Delta^{n} \times \mathbb{A}^{q}\right)\right|
$$

$4^{\mathrm{e}}$ SÉRIE - TOME $35-2002-\mathrm{N}^{\circ} 6$ 
which are compatible with maps of the corresponding spaces defined by strictly increasing $\theta:[m] \rightarrow[n]$ and hence define a map on Segal realizations

$$
\left\|\mathrm{I}_{\bullet} \times\left(n \mapsto\left|S_{\bullet}^{\Phi} \mathcal{C}^{q}\left(\Delta^{n} \times \mathbb{A}^{1} \times \mathbb{A}^{q}\right)\right|\right)\right\| \stackrel{H}{\longrightarrow}\left\|n \mapsto\left|S_{\bullet}^{\mathcal{C}^{q}}\left(\Delta^{n} \times \mathbb{A}^{q}\right)\right|\right\| .
$$

Since for any simplicial space $Z_{\bullet}$ the obvious embeddings

$$
\left\|Z_{\bullet}\right\| \rightrightarrows\left\|\mathrm{I}_{\bullet} \times Z_{\bullet}\right\|
$$

defined by points 0 and 1 are homotopic, we conclude the commutativity up to homotopy of the following diagrams.

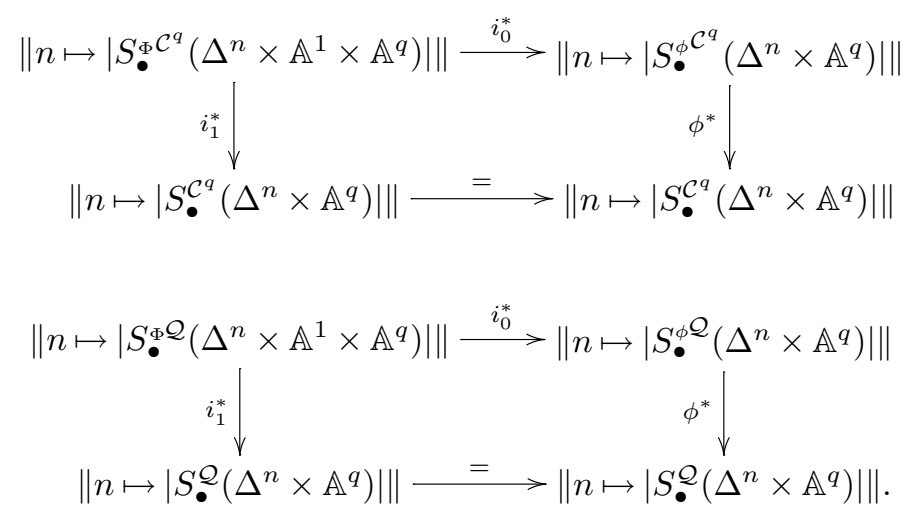

Observe that the compositions $p \circ i_{0}$ and $p \circ i_{1}$ coincide with the identity morphism. Thus, we conclude that ${ }_{\Phi} \mathcal{C}^{q}\left(\Delta^{n} \times \mathbb{A}^{q}\right) \subset{ }_{\phi} \mathcal{C}^{q}\left(\Delta^{n} \times \mathbb{A}^{q}\right)$ and that the restriction of $\phi^{*}$ to $\left\|n \mapsto\left|S_{\bullet}^{\Phi} \mathcal{C}^{q}\left(\Delta^{n} \times \mathbb{A}^{q}\right)\right|\right\|$ is homotopic to the inclusion map

$$
\left\|n \mapsto\left|{ }_{\Phi} \mathcal{C}^{q}\left(\Delta^{n} \times \mathbb{A}^{q}\right)\right|\right\| \hookrightarrow\left\|n \mapsto\left|\mathcal{C}^{q}\left(\Delta^{n} \times \mathbb{A}^{q}\right)\right|\right\| .
$$

Similarly, ${ }_{\Phi} \mathcal{Q}\left(\Delta^{n} \times \mathbb{A}^{q}\right) \subset{ }_{\phi} \mathcal{Q}\left(\Delta^{n} \times \mathbb{A}^{q}\right)$ and the restriction of $\phi^{*}$ to $\left\|n \mapsto\left|S_{\bullet}^{\Phi} \mathcal{Q}\left(\Delta^{n} \times \mathbb{A}^{q}\right)\right|\right\|$ is homotopic to the inclusion map $\left\|n \mapsto\left|{ }_{\Phi} \mathcal{Q}\left(\Delta^{n} \times \mathbb{A}^{q}\right)\right|\right\| \hookrightarrow\left\|n \mapsto\left|\mathcal{Q}\left(\Delta^{n} \times \mathbb{A}^{q}\right)\right|\right\|$.

The proof is now completed by appealing to Proposition 8.4.

The preceding results in conjunction with Theorem 7.6 now provide us with the following theorem.

THEOREM 8.6. - The embeddings of $\Omega$-prespectra

$$
\mathcal{K}^{\mathcal{Q}, \mathbb{A}^{q}}\left(\Delta^{\bullet}\right) \hookrightarrow \mathcal{K}^{\mathcal{C}^{q}}\left(\Delta^{\bullet} \times \mathbb{A}^{q}\right) \hookleftarrow \mathcal{K}^{\mathcal{C}^{q}}\left(\Delta^{\bullet}\right)
$$

are weak equivalences.

Proof. - Theorem 7.6 asserts that the right embedding is a weak equivalence. Corollary 8.3 and Proposition 8.5 show easily that the embedding of spaces

$$
\left\|n \mapsto\left|S_{\bullet}^{\mathcal{Q}}\left(\Delta^{n} \times \mathbb{A}^{q}\right)\right|\right\| \hookrightarrow\left\|n \mapsto\left|S_{\bullet}^{\mathcal{C}^{q}}\left(\Delta^{n} \times \mathbb{A}^{q}\right)\right|\right\|
$$

is a weak equivalence. Since the natural projection from the Segal realization to the usual one is also an equivalence, we conclude that the embedding 


$$
\begin{aligned}
|n \mapsto| S_{\bullet}^{\mathcal{Q}}\left(\Delta^{n} \times \mathbb{A}^{q}\right)|| & =\left|S_{\bullet}^{\mathcal{Q}}\left(\Delta^{\bullet} \times \mathbb{A}^{q}\right)\right| \hookrightarrow|n \mapsto| S_{\bullet}^{\mathcal{C}^{q}}\left(\Delta^{n} \times \mathbb{A}^{q}\right)|| \\
& =\left|S_{\bullet}^{\mathcal{C}^{q}}\left(\Delta^{\bullet} \times \mathbb{A}^{q}\right)\right|
\end{aligned}
$$

is a weak equivalence as well.

Consistent with our notation $\mathcal{K}^{\mathcal{Q}, S}(X)$, we shall denote by $\mathbb{Z}^{\mathcal{Q}, S}(X)$ the free abelian group generated by closed integral subschemes $Z \subset X \times S$, which are quasifinite and dominant over some connected component of $X$. The presheaf $X \mapsto \mathbb{Z}^{\mathcal{Q}, S}(X)$ is a presheaf with transfers in the sense of Voevodsky [34], and is also a sheaf in the etale topology as can be seen using faithfully flat descent. This etale sheaf with transfers is a special case of the sheaf of equidimensional cycles studied in [10], where it was written $z_{\text {equi }}(S, 0)(X)$.

LEMMA 8.7. - (1) For any $X, S \in S m / F$ there exists a natural surjective homomorphism $K_{0}^{\mathcal{Q}, S}(X) \rightarrow \mathbb{Z}^{\mathcal{Q}, S}(X)$.

(2) In case $X=\mathbb{A}^{n}$ the above homomorphism is an isomorphism.

Proof. - The $K$-groups $K_{*}^{\mathcal{Q}, S}(X)$ coincide with the $K$-groups of an abelian category $\mathcal{M}$, consisting of coherent sheaves $\mathcal{F}$ on $S \times X$ such that $\operatorname{Supp} \mathcal{F} \in \mathcal{Q}_{X}(S \times X)$ - see [32]. Let $\mathcal{M}^{\prime}$ denote the Serre subcategory of $\mathcal{M}$ consisting of sheaves $\mathcal{F} \in \mathcal{M}$ whose support is not dominant over a component of $X$. The quotient abelian category $\mathcal{M} / \mathcal{M}^{\prime}$ may be identified with $\coprod_{z} M f l\left(\mathcal{O}_{z}\right)$, where $M f l$ stands for the category of modules of finite length and $z$ in the coproduct runs throughout the set of generic points of closed integral subschemes $Z \subset S \times X$, which are quasifinite and dominant over a component of $X$. According to the devissage Theorem [25] the group $K_{0}\left(\coprod_{z} M f l\left(\mathcal{O}_{z}\right)\right)$ coincides with $\mathbb{Z}^{\mathcal{Q}, S}(X)$, which immediately provides us with the desired natural surjective homomorphism.

To show that this homomorphism is an isomorphism in case $X=\mathbb{A}^{n}$ one has to show that in this case the embedding $\mathcal{M}^{\prime} \hookrightarrow \mathcal{M}$ induces a zero map on $K_{0}$-groups. The proof of this last fact is based on the use of the linear version of Quillen's trick and proceeds as follows (cf. proof of $[15,1.2])$.

Let $M$ be a coherent sheaf on $\mathbb{A}^{n} \times S$ whose support $Z=S u p p M$ is quasifinite but not dominant over $\mathbb{A}^{n}$. Denote by $Y$ the closure of the image of $Z$ in $\mathbb{A}^{n}$. Since $\operatorname{dim} Y<n$ there exists a non-zero polynomial $P \in F\left[X_{1}, \ldots, X_{n}\right]$ vanishing on $Y$. Making a change of variables we may even assume that $P$ is monic in $X_{n}$. Denote by $p: \mathbb{A}^{n} \times S \rightarrow \mathbb{A}^{n-1} \times S$ the projection onto the first $n-1$ coordinates. Since a sufficiently high power of $P$ annihilates $M$, we conclude easily that $p_{*}(M)$ is a coherent sheaf on $\mathbb{A}^{n-1} \times S$, with support quasifinite over $\mathbb{A}^{n-1}$. Multiplication by $X_{n}$ determines an endomorphism $\alpha$ of $p_{*}(M)$. Finally we use the well-known characteristic exact sequence of an endomorphism (see [1], Chapter 12)

$$
0 \rightarrow p^{*}\left(p_{*}(M)\right) \stackrel{X_{n}-p^{*}(\alpha)}{\longrightarrow} p^{*}\left(p_{*}(M)\right) \rightarrow M \rightarrow 0
$$

to conclude that $[M]=\left[p^{*}\left(p_{*}(M)\right)\right]-\left[p^{*}\left(p_{*}(M)\right)\right]=0 \in K_{0}(\mathcal{M})$.

LEMMA 8.7.1. - Let $S, S^{\prime} \in S m / F$ be equidimensional schemes with $\operatorname{dim} S^{\prime}<\operatorname{dim} S$. Let further $X \in S m / F$ be a scheme and $f: X \times S^{\prime} \rightarrow X \times S$ be a quasifinite morphism over $X$. Then the induced homomorphism

$$
K_{0}^{\mathcal{Q}, S}(X) \stackrel{f^{*}}{\longrightarrow} K_{0}^{\mathcal{Q}, S^{\prime}}(X) \rightarrow \mathbb{Z}^{\mathcal{Q}, S^{\prime}}(X)
$$

is trivial. 
Proof. - We may obviously assume that $X$ is irreducible. As was noted above the group $K_{0}^{\mathcal{Q}, S}(X)$ coincides with $K_{0}(\mathcal{M})$, where $\mathcal{M}$ is the abelian category of coherent sheaves on $X \times S$ with support quasifinite over $X$ - see [32]. For any $M \in \mathcal{M}$ denote by $[M]$ its class in $K_{0}^{\mathcal{Q}, S}(X)=K_{0}(\mathcal{M})$. To compute the image of $[M]$ under $f^{*}$ one should consider a resolution

$$
0 \leftarrow M \leftarrow P_{0} \leftarrow P_{1} \leftarrow \cdots \leftarrow P_{n} \leftarrow 0
$$

of $M$ by vector bundles, apply $f^{*}$ to $P_{\bullet}$ and take the alternating sum of classes of the homology sheaves of $f^{*}\left(P_{\bullet}\right)$. In other words the inverse image is given by the usual Tor-formula

$$
f^{*}([M])=\sum_{i=0}^{\infty}(-1)^{i}\left[\operatorname{Tor}_{i}^{\mathcal{O}_{X \times S}}\left(\mathcal{O}_{X \times S^{\prime}}, M\right)\right] .
$$

Let $Z^{\prime} \subset X \times S^{\prime}$ be a closed integral subscheme quasifinite and dominant over $X$, let $z^{\prime}$ be the generic point of $Z^{\prime}$ and let $z=f\left(z^{\prime}\right)$ denote the image of $z^{\prime}$ in $X \times S$. The multiplicity with which $Z^{\prime}$ appears in the image of $f^{*}([M])$ in $\mathbb{Z}^{\mathcal{Q}, S^{\prime}}(X)$ coincides with

$$
\begin{aligned}
& \sum_{i=0}^{\infty}(-1)^{i} l_{\mathcal{O}_{X \times S^{\prime}, z^{\prime}}}\left(\operatorname{Tor}_{i}^{\mathcal{O}_{X \times S}}\left(\mathcal{O}_{X \times S^{\prime}}, M\right)_{z^{\prime}}\right) \\
& \quad=\left[F\left(z^{\prime}\right): F(z)\right]^{-1} \cdot \sum_{i=0}^{\infty}(-1)^{i} l_{\mathcal{O}_{X \times S, z}}\left(\operatorname{Tor}_{i}^{\mathcal{O}_{X \times S, z}}\left(\mathcal{O}_{X \times S^{\prime}, z^{\prime}}, M_{z}\right)\right) .
\end{aligned}
$$

The last expression is zero since

$$
\begin{aligned}
\operatorname{dim} \mathcal{O}_{X \times S^{\prime}, z^{\prime}}+\operatorname{dim} M_{z} & \leqslant \operatorname{dim} X+\operatorname{dim} S^{\prime}-\operatorname{dim} Z^{\prime}+\operatorname{dim} \text { Supp } M-\operatorname{dim} Z \\
& =\operatorname{dim} S^{\prime}+\operatorname{dim} \text { Supp } M-\operatorname{dim} X \\
& \leqslant \operatorname{dim} S^{\prime}<\operatorname{dim} S=\operatorname{dim} X+\operatorname{dim} S-\operatorname{dim} Z=\operatorname{dim} \mathcal{O}_{X \times S, z}
\end{aligned}
$$

see [27], Chapter V(C.1), §3.

Since $X \mapsto \mathcal{K}^{\mathcal{Q}, S}(X)$ is a contravariant functor from $S m / F$ to the category of prespectra, we may define for any cosimplicial scheme $X^{\bullet}$ the prespectrum

$$
\mathcal{K}^{\mathcal{Q}, S}\left(X^{\bullet}\right) \equiv\left|n \mapsto \mathcal{K}^{\mathcal{Q}, S}\left(X^{n}\right)\right|
$$

In particular for any $X \in S m / F$,we shall consider the $\Omega$-prespectrum

$$
\mathcal{K}^{\mathcal{Q}, \mathbb{A}^{q}}\left(X \times \Delta^{\bullet}\right) \equiv\left|n \mapsto \mathcal{K}^{\mathcal{Q}, \mathbb{A}^{q}}\left(X \times \Delta^{n}\right)\right| .
$$

Note further that every quasifinite morphism $S^{\prime} \rightarrow S$ defines a natural (in $X$ ) morphism of prespectra

$$
\mathcal{K}^{\mathcal{Q}, S}\left(\Delta^{\bullet} \times X\right) \rightarrow \mathcal{K}^{\mathcal{Q}, S^{\prime}}\left(\Delta^{\bullet} \times X\right)
$$

In particular we have a natural (in $X$ ) morphism of prespectra

$$
\mathcal{K}^{\mathcal{Q}, \mathbb{A}^{q+1}}\left(\Delta^{\bullet} \times X\right) \rightarrow \mathcal{K}^{\mathcal{Q}, \mathbb{A}^{q}}\left(\Delta^{\bullet} \times X\right),
$$

corresponding to the embedding $i_{0}: \mathbb{A}^{q} \hookrightarrow \mathbb{A}^{q+1}=\mathbb{A}^{q} \times \mathbb{A}^{1}$ defined by the point $0 \in \mathbb{A}^{1}$. 
Setting $C_{n}\left(\mathbb{Z}^{\mathcal{Q}, S}\right)(X)=\mathbb{Z}^{\mathcal{Q}, S}\left(\Delta^{n} \times X\right)$ we get a simplicial sheaf $C_{\bullet}\left(\mathbb{Z}^{\mathcal{Q}, S}\right)$. In view of Lemma 8.7, the construction of (C.1.1) provides us with a canonical morphism of prespectra

$$
\mathcal{K}^{\mathcal{Q}, \mathbb{A}^{q}}\left(\Delta^{\bullet}\right) \rightarrow \mathbb{B}\left(C \bullet\left(\mathbb{Z}^{\mathcal{Q}, \mathbb{A}^{q}}\right)(\operatorname{Spec} F)\right)
$$

Lemma 8.7.1 shows that the composition

$$
\mathcal{K}^{\mathcal{Q}, \mathbb{A}^{q+1}}\left(\Delta^{\bullet}\right) \rightarrow \mathcal{K}^{\mathcal{Q}, \mathbb{A}^{q}}\left(\Delta^{\bullet}\right) \rightarrow \mathbb{B}\left(C \bullet\left(\mathbb{Z}^{\mathcal{Q}, \mathbb{A}^{q}}\right)(\operatorname{Spec} F)\right)
$$

is trivial.

LEMMA 8.8. - Let $\mathcal{Q}_{0}\left(\Delta^{n} \times \mathbb{A}^{q+1}\right)$ denote the family of supports on $\Delta^{n} \times \mathbb{A}^{q+1}$ consisting of those closed subschemes $Y \in \mathcal{Q}\left(\Delta^{n} \times \mathbb{A}^{q+1}\right)$ whose intersection with $\Delta^{n} \times \mathbb{A}^{q}$ belongs to $\mathcal{C}^{q+1}\left(\Delta^{n} \times \mathbb{A}^{q}\right)$. Then the natural morphisms of prespectra

$$
\begin{gathered}
\mathcal{K}^{\mathcal{Q}_{0}, \mathbb{A}^{q+1}}\left(\Delta^{\bullet}\right) \rightarrow \mathcal{K}^{\mathcal{Q}, \mathbb{A}^{q+1}}\left(\Delta^{\bullet}\right), \\
\mathcal{K}^{\mathcal{Q}_{0}, \mathbb{A}^{q+1}}\left(\Delta^{\bullet}\right) \rightarrow \mathcal{K}^{\mathcal{C}^{q+1}}\left(\Delta^{\bullet} \times \mathbb{A}^{q}\right)
\end{gathered}
$$

are weak equivalences.

Proof. - Essentially the same argument as in the proof of Corollary 7.10 establishes that the first embedding is a weak equivalence. To show that the second morphism is a weak equivalence, we utilize the family of supports $\mathcal{C}_{0}^{q+1}\left(\mathbb{A}^{q} \times \mathbb{A}^{1} \times \Delta^{n}\right)$ as introduced in (7.2.3). Observe that this second morphism coincides with the composition

$$
\mathcal{K}^{\mathcal{Q}_{0}, \mathbb{A}^{q+1}}\left(\Delta^{\bullet}\right) \rightarrow \mathcal{K}^{\mathcal{C}_{0}^{q+1}}\left(\mathbb{A}^{q} \times \mathbb{A}^{1} \times \Delta^{\bullet}\right) \stackrel{s_{0}}{\longrightarrow} \mathcal{K}^{\mathcal{C}^{q+1}}\left(\Delta^{\bullet} \times \mathbb{A}^{q}\right)
$$

where the first arrow is induced by the obvious inclusion of families of supports and the second arrow is the specialization at 0 morphism considered in Section 7 . The first equivalence of the present lemma together with Corollary 7.10 and Theorem 8.6 imply that the first arrow above is a weak equivalence, whereas Theorem 7.6 in conjunction with Corollary 7.10 show that the second arrow is an equivalence as well.

THEOREM 8.9. - The sequence of maps

$$
\mathcal{K}^{\mathcal{Q}, \mathbb{A}^{q+1}}\left(\Delta^{\bullet}\right) \rightarrow \mathcal{K}^{\mathcal{Q}, \mathbb{A}^{q}}\left(\Delta^{\bullet}\right) \rightarrow \mathbb{B}\left(C \bullet\left(\mathbb{Z}^{\mathcal{Q}, \mathbb{A}^{q}}\right)(F)\right)
$$

is a homotopy fibration sequence.

Proof. - By Lemma 8.8, it suffices to show that the sequence

$$
\mathcal{K}^{\mathcal{Q}_{0}, \mathbb{A}^{q+1}}\left(\Delta^{\bullet}\right) \rightarrow \mathcal{K}^{\mathcal{Q}, \mathbb{A}^{q}}\left(\Delta^{\bullet}\right) \rightarrow \mathbb{B}\left(C \bullet\left(\mathbb{Z}^{\mathcal{Q}, \mathbb{A}^{q}}\right)(F)\right)
$$

is a homotopy fibration sequence. Consider the following commutative diagram

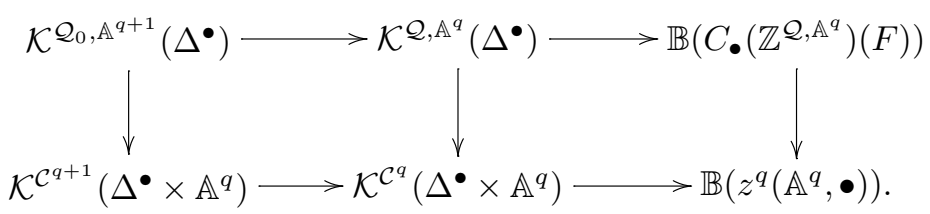


By Lemma 8.8, the left vertical map is a weak equivalence, Theorem 8.6 implies that the middle vertical arrow is a weak equivalence, and [29] verifies that the right vertical arrow is also a weak equivalence. Since the bottom row is a homotopy fibration sequence by Corollary 7.7 , the top row is likewise a homotopy fibration sequence.

The embedding $\mathbb{A}^{q} \hookrightarrow \mathbb{A}^{q+1}$, used above to define the morphism of prespectra

$$
\mathcal{K}^{\mathcal{Q}, \mathbb{A}^{q+1}}\left(\Delta^{\bullet}\right) \rightarrow \mathcal{K}^{\mathcal{Q}, \mathbb{A}^{q}}\left(\Delta^{\bullet}\right)
$$

is by no means essential for our construction. The following result shows that we can replace it by any other coordinate embedding (i.e., embeddings of the form

$$
\left.\left(x_{1}, \ldots, x_{q}\right) \mapsto\left(x_{1}, \ldots, \underset{k}{0}, \ldots, x_{q}\right)\right) .
$$

LEMMA 8.10. - Let $i, i^{\prime}: \mathbb{A}^{q} \rightarrow \mathbb{A}^{q+1}$ be two coordinate embeddings. For any scheme $X \in S m / F$ the corresponding morphisms of prespectra $\mathcal{K}^{\mathcal{Q}, \mathbb{A}^{q+1}}\left(\Delta^{\bullet} \times X\right) \rightarrow \mathcal{K}^{\mathcal{Q}, \mathbb{A}^{q}}\left(\Delta^{\bullet} \times X\right)$ are homotopic. Moreover the corresponding homotopy becomes constant when composed with the morphism $\mathcal{K}^{\mathcal{Q}, \mathbb{A}^{q}}\left(\Delta^{\bullet} \times X\right) \rightarrow \mathbb{B}\left(C_{\bullet}\left(\mathbb{Z}^{\mathcal{Q}, \mathbb{A}^{q}}\right)(X)\right)$ and hence the resulting morphisms

$$
\mathcal{K}^{\mathcal{Q}, \mathbb{A}^{q+1}}\left(\Delta^{\bullet} \times X\right) \rightarrow f i b\left\{\mathcal{K}^{\mathcal{Q}, \mathbb{A}^{q}}\left(\Delta^{\bullet} \times X\right) \rightarrow \mathbb{B}\left(C \bullet\left(\mathbb{Z}^{\mathcal{Q}, \mathbb{A}^{q}}\right)(X)\right)\right\}
$$

are also homotopic.

Proof. - It suffices to consider the case when the corresponding indices $k$ differ by 1 . In this case one checks easily that the morphism

$$
\eta\left(i, i^{\prime}\right) \times i d_{\mathbb{A}^{1}}: \mathbb{A}^{q} \times \mathbb{A}^{1} \rightarrow \mathbb{A}^{q+1} \times \mathbb{A}^{1} \quad(x, t) \mapsto\left(t \cdot i(x)+(1-t) \cdot i^{\prime}(x), t\right)
$$

is quasifinite and our statement follows from the following (more general) fact.

LEMMA 8.10.1.- Let $i, i^{\prime}: \mathbb{A}^{q} \rightarrow \mathbb{A}^{q+1}$ be two quasifinite morphisms for which the above morphism

$$
\eta\left(i, i^{\prime}\right) \times i d_{\mathbb{A}^{1}}: \mathbb{A}^{q} \times \mathbb{A}^{1} \rightarrow \mathbb{A}^{q+1} \times \mathbb{A}^{1}
$$

is quasifinite as well. Then for any scheme $X \in S m / F$ the corresponding morphisms of prespectra $\mathcal{K}^{\mathcal{Q}, \mathbb{A}^{q+1}}\left(\Delta^{\bullet} \times X\right) \rightarrow \mathcal{K}^{\mathcal{Q}, \mathbb{A}^{q}}\left(\Delta^{\bullet} \times X\right)$ are canonically homotopic and the corresponding homotopy becomes trivial when composed with the morphism

$$
\mathcal{K}^{\mathcal{Q}, \mathbb{A}^{q}}\left(\Delta^{\bullet} \times X\right) \rightarrow \mathbb{B}\left(C \bullet\left(\mathbb{Z}^{\mathcal{Q}, \mathbb{A}^{q}}\right)(X)\right) .
$$

Proof. - Consider the following diagram of prespectra

$$
\mathcal{K}^{\mathcal{Q}, \mathbb{A}^{q+1}}\left(\Delta^{\bullet} \times X\right) \rightarrow \mathcal{K}^{\mathcal{Q}, \mathbb{A}^{q}}\left(\Delta^{\bullet} \times X \times \mathbb{A}^{1}\right) \rightrightarrows \mathcal{K}^{\mathcal{Q}, \mathbb{A}^{q}}\left(\Delta^{\bullet} \times X\right)
$$

Here the first arrow is induced by the morphism $\eta\left(i, i^{\prime}\right): \mathbb{A}^{q} \times \mathbb{A}^{1} \rightarrow \mathbb{A}^{q+1}$ and the last two arrows are pull-backs corresponding to the closed embeddings $X \rightarrow X \times \mathbb{A}^{1}$ defined by the closed points $0,1 \in \mathbb{A}^{1}$. Now it suffices to note that the two morphisms in question coincide with the top and bottom compositions of the above morphisms and use Lemma 7.1. The fact that the resulting homotopy becomes trivial being composed with $\mathcal{K}^{\mathcal{Q}, \mathbb{A}^{q}}\left(\Delta^{\bullet} \times X\right) \rightarrow \mathbb{B}\left(C_{\bullet}\left(\mathbb{Z}^{\mathcal{Q}, \mathbb{A}^{q}}\right)(X)\right)$ follows easily from the explicit form of the homotopy, using Lemma 8.7.1. 
Consider in particular the special case where $i=i_{q}, i^{\prime}=i_{q-1}$ are two coordinate embeddings (so that $i^{*}$ is the standard morphism used before). Let $F_{t}$ denote the homotopy relating the morphisms $i_{q}^{*}$ and $i_{q-1}^{*}$. In Section 14 we will need the following property of this homotopy.

LEMMA 8.10.2. - The homotopy $F_{t}$ becomes constant when composed with the morphism $\mathcal{K}^{\mathcal{Q}, \mathbb{A}^{q}}\left(\Delta^{\bullet} \times X\right) \rightarrow \mathcal{K}^{\mathcal{Q}, \mathbb{A}^{q-1}}\left(\Delta^{\bullet} \times X\right)$.

Proof. - Note that the composition

$$
\mathbb{A}^{q-1} \times \mathbb{A}^{1} \rightarrow \mathbb{A}^{q} \times \mathbb{A}^{1} \stackrel{\eta\left(i, i^{\prime}\right)}{\longrightarrow} \mathbb{A}^{q+1}
$$

coincides with the composition $\mathbb{A}^{q-1} \times \mathbb{A}^{1} \stackrel{p}{\rightarrow} \mathbb{A}^{q-1} \hookrightarrow \mathbb{A}^{q+1}$ since $i=i^{\prime}$ on $\mathbb{A}^{q-1}$. This implies readily the commutativity of the diagram

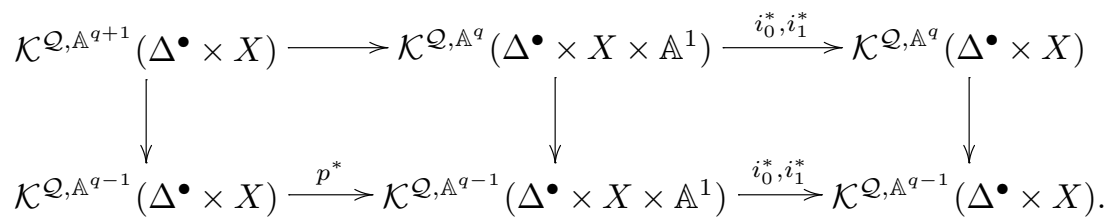

Our result follows now from Remark 7.1.1.

\section{Transfers in homotopy groups of prespectra $\mathcal{K}^{\mathcal{C}^{q}}\left(\Delta^{\bullet} \times X\right)$ and $\mathcal{K}^{\mathcal{Q}, \mathbb{A}^{q}}\left(\Delta^{\bullet} \times X\right)$}

In this section we define transfers in the homotopy groups of the prespectra in the title of the section corresponding to finite flat morphisms. This construction will be crucial in Section 11. Here we use it to extend the results of the two previous sections to the case of finite fields.

Let $p: D \rightarrow S$ be a finite flat morphism of schemes. Consider the functor

$$
p_{*}: \mathcal{P}(S c h / D) \rightarrow \mathcal{P}(S c h / S)
$$

on categories of "big vector bundles" (see Appendix C.4). This functor is defined uniquely up to a unique isomorphism, but not quite uniquely as yet. To make it absolutely well-defined we have to fix models for all schemes $S^{\prime} \times{ }_{S} D\left(S^{\prime} \in S c h / S\right)$. As soon as these choices are made the required big vector bundle is defined uniquely by the formula

$$
p_{*}(P)\left(S^{\prime}\right)=P\left(D \times{ }_{S} S^{\prime}\right) .
$$

Here $P: S c h / D \rightarrow A b$ is a big vector bundle on the site $S c h / D$ and the resulting sheaf of $\mathcal{O}$ modules on the site $S c h / S$ is a big vector bundle since the morphism $p: D \rightarrow S$ is finite and flat. Let now $S^{\prime} \rightarrow S$ be a scheme of finite type over $S$. Proceeding in the same way as above we may consider the functor $p_{*}^{\prime}: \mathcal{P}\left(S c h / D \times{ }_{S} S^{\prime}\right) \rightarrow \mathcal{P}\left(S c h / S^{\prime}\right)$. Note however that by now we don't need to make any choices, since for any $S^{\prime \prime} / S^{\prime}$ the scheme $S^{\prime \prime} \times{ }_{S} D$ already fixed above may (and will) be chosen as the model for $\left(D \times{ }_{S} S^{\prime}\right) \times{ }_{S^{\prime}} S^{\prime \prime}$. With this agreement we see immediately that the following statement holds. 
LEMma 9.1. - Consider a Cartesian diagram of schemes as above

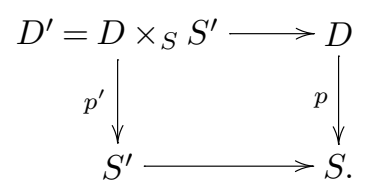

Then the following diagram of functors strictly commutes.

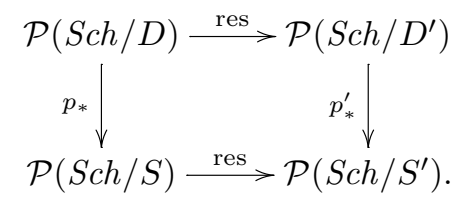

Here the horizontal arrows are the inverse image (restriction of the domain) functors and the vertical arrows are the direct image functors as fixed above.

We shall use the notation $\operatorname{Tr}_{D / S}, \operatorname{Tr}_{D^{\prime} / S^{\prime}}$ for the functors $p_{*}, p_{*}^{\prime}$ introduced above. Lemma 9.1 implies immediately the following corollary

COROLlaRY 9.2. - Let $\theta:[m] \rightarrow[n]$ be a non-decreasing map and let the same letter $\theta$ be used to denote the corresponding morphism of schemes $\Delta^{m} \rightarrow \Delta^{n}$. For any $q \geqslant 0$ the following diagram of functors strictly commutes

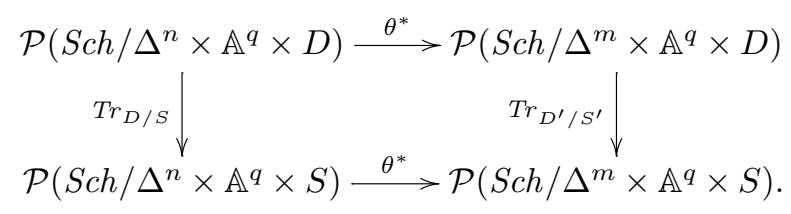

Corollary 9.2 implies readily that for any $q \geqslant 0$ we get natural morphisms of simplicial prespectra (where in the second case we assume that the scheme $S$, and hence also $D$, is equidimensional)

$$
\begin{aligned}
& \operatorname{Tr}_{D / S}: \mathcal{K}^{\mathcal{Q}, \mathbb{A}^{q}}\left(\Delta^{\bullet} \times D\right) \rightarrow \mathcal{K}^{\mathcal{Q}, \mathbb{A}^{q}}\left(\Delta^{\bullet} \times S\right), \\
& \operatorname{Tr}_{D / S}: \mathcal{K}^{\mathcal{C}^{q}}\left(\Delta^{\bullet} \times D\right) \rightarrow \mathcal{K}^{\mathcal{C}^{q}}\left(\Delta^{\bullet} \times S\right) .
\end{aligned}
$$

Moreover one checks easily that if we change the choices for the fiber products which were made to produce the above map then the corresponding maps

$$
\begin{aligned}
& \operatorname{Tr}_{D / S}: \mathcal{K}^{\mathcal{Q}, \mathbb{A}^{q}}\left(\Delta^{n} \times D\right) \rightarrow \mathcal{K}^{\mathcal{Q}, \mathbb{A}^{q}}\left(\Delta^{n} \times S\right), \\
& \operatorname{Tr}_{D / S}: \mathcal{K}^{\mathcal{C}^{q}}\left(\Delta^{\bullet} \times D\right) \rightarrow \mathcal{K}^{\mathcal{C}^{q}}\left(\Delta^{\bullet} \times S\right)
\end{aligned}
$$

are replaced by homotopic ones and, in particular, the induced homomorphisms in homotopy groups are independent of the choices made. We keep the same notation $\operatorname{Tr}_{D / S}$ for the maps in homotopy groups of the above prespectra induced by the morphisms of prespectra $\operatorname{Tr}_{D / S}$.

In the case of the prespectrum $\mathcal{K}^{\mathcal{Q}, \mathbb{A}^{q}}\left(\Delta^{n} \times X\right)$, which depends contravariantly functorially on $X$ (thanks to the functorial constructions of Appendix C), the transfer maps are compatible with pull-backs. The following statement follows immediately from Lemma 9.1. 
Lemma 9.3. - Let $f: S^{\prime} \rightarrow S$ be a scheme of finite type over $S$. Consider the Cartesian diagram

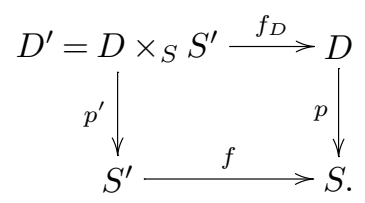

Then the following diagram of simplicial prespectra commutes

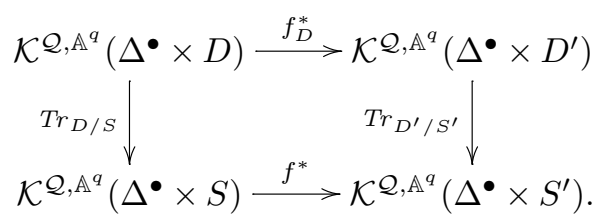

PROPOSITION 9.4. - Assume that the vector bundle $p_{*}\left(\mathcal{O}_{D}\right)$ is a free $\mathcal{O}_{S}$-module of rank $n$. Then the compositions

$$
\begin{aligned}
& \pi_{i}\left(\mathcal{K}^{\mathcal{Q}, \mathbb{A}^{q}}\left(\Delta^{\bullet} \times S\right)\right) \stackrel{p^{*}}{\longrightarrow} \pi_{i}\left(\mathcal{K}^{\mathcal{Q}, \mathbb{A}^{q}}\left(\Delta^{\bullet} \times D\right)\right) \stackrel{\operatorname{Tr}_{D / S}}{\longrightarrow} \pi_{i}\left(\mathcal{K}^{\mathcal{Q}, \mathbb{A}^{q}}\left(\Delta^{\bullet} \times S\right)\right), \\
& \pi_{i}\left(\mathcal{K}^{\mathcal{C}^{q}}\left(\Delta^{\bullet} \times S\right)\right) \stackrel{p^{*}}{\longrightarrow} \pi_{i}\left(\mathcal{K}^{\mathcal{C}^{q}}\left(\Delta^{\bullet} \times D\right)\right) \stackrel{\operatorname{Tr}_{D / S}}{\longrightarrow} \pi_{i}\left(\mathcal{K}^{\mathcal{C}^{q}}\left(\Delta^{\bullet} \times S\right)\right)
\end{aligned}
$$

coincide with multiplication by $n$.

Proof. - This follows easily from the Waldhausen Additivity Theorem [37] in view of the fact that the composition functor $p_{*} \circ p^{*}$ is naturally isomorphic to the direct sum of $n$ copies of the identity functor.

As a first application of the existence of the transfer maps we show that the results of the previous two sections remain valid over finite fields. To do this, we need one more elementary technical result.

Lemma 9.5. - Let $E / F$ be an algebraic extension of fields. Then for any scheme $X \in S m / F$ we have natural isomorphisms

$$
\begin{aligned}
& K_{i}^{\mathcal{Q}, \mathbb{A}^{q}}\left(\Delta^{\bullet} \times X_{E}\right)=\underset{L}{\lim _{\longrightarrow}} K_{i}^{\mathcal{Q}, \mathbb{A}^{q}}\left(\Delta^{\bullet} \times X_{L}\right), \\
& K_{i}^{\mathcal{C}^{q}}\left(\Delta^{\bullet} \times X_{E}\right)=\underset{L}{\lim _{\longrightarrow}} K_{i}^{\mathcal{C}^{q}}\left(\Delta^{\bullet} \times X_{L}\right),
\end{aligned}
$$

where $L$ runs through all finite subextensions of $E / F$.

THEOREM 9.6. - The results Theorems 7.6, 8.6, 8.9 are valid over finite fields.

Proof. - To show how to extend Theorem 7.6 to the case of finite fields let $F$ be a finite field and let $X / F$ be a smooth scheme over $F$. We have to show that the homomorphisms

$$
K_{i}^{\mathcal{C}^{q}}\left(\Delta^{\bullet} \times X\right) \stackrel{p^{*}}{\longrightarrow} K_{i}^{\mathcal{C}^{q}}\left(\Delta^{\bullet} \times \mathbb{A}^{1} \times X\right)
$$

are isomorphisms. We shall show that these maps are injective, surjectivity is proved similarly. Assume that $a \in K_{i}^{\mathcal{C}^{q}}\left(\Delta^{\bullet} \times X\right)$ is in the kernel of $p^{*}$. For any infinite algebraic extension $E / F$ the image of $a$ in $K_{i}^{\mathcal{C}^{q}}\left(\Delta^{\bullet} \times X_{E}\right)$ is trivial in view of Theorem 7.6. We conclude further from 
Lemma 9.5 that there exists a finite subextension $F \subset L \subset E$ such that $a$ dies in $K_{i}^{\mathcal{C}^{q}}\left(\Delta^{\bullet} \times X_{L}\right)$ and hence is killed by $[L: F]$ according to Proposition 9.4. Finally for any prime integer $l \neq \operatorname{char} F$ we can find an infinite extension $E / F$ with Galois group $\operatorname{Gal}(E / F)=\mathbb{Z}_{l}$, in which case the degree $[L: F]$ is a power of $l$ (for any finite subextension $L$ ). Thus $a$ is killed by a sufficiently high power of an arbitrary prime integer and hence $a=0$.

Theorem 8.6 is proved in exactly the same way. Theorem 8.9 follows similarly, by observing that its statement is verified by showing that the natural map from $\mathcal{K}^{\mathcal{Q}, \mathbb{A}^{q+1}}\left(\Delta^{\bullet}\right)$ to the homotopy fibre is a weak equivalence.

\section{Pseudo pretheories}

Vladimir Voevodsky has introduced the concept of a pretheory which has proved extremely useful in the study of several problems. A pretheory on the category $S m / F$ is a contravariant functor which has well-behaved transfers. A key property of a homotopy invariant pretheory $\mathcal{F}$ is the fact that the restriction map $\mathcal{F}(S) \rightarrow \mathcal{F}(F(S))$ is injective for any smooth affine irreducible semilocal scheme $S$ with field of rational functions $F(S)$; in particular, $\mathcal{F}$ vanishes on such semilocal schemes whenever it vanishes on all fields.

In order to apply this to extend the fibration sequence of Theorem 6.1 from fields to semilocal schemes, we need a minor modification of Voevodsky's pretheories. Namely, the functors $X \mapsto \pi_{n}\left(\mathcal{K}^{\mathcal{Q}, \mathbb{A}^{q}}\left(\Delta^{\bullet} \times X\right)\right)$ are not quite pretheories with the given transfers $\operatorname{Tr}_{D / S}$ because $\operatorname{Tr}_{\left(D \cdot D^{\prime}\right) / S}$ is not necessarily equal to $\operatorname{Tr}_{D / S}+\operatorname{Tr}_{D^{\prime} / S}$. (Here, we are identifying an effective Cartier divisor $D$ with a codimension 1 subscheme, so that $D \cdot D^{\prime}$ denotes the subscheme associated to the product of Cartier divisors.) The purpose of this section is to introduce the slightly weaker notion of a pseudo pretheory and to verify that minor modifications of Voevodsky's arguments imply that the above injectivity property remains valid for such pseudo pretheories.

We say that a contravariant functor $\mathcal{F}$ from the category of smooth schemes over a given field $F$ to abelian groups is a pseudo pretheory if $\mathcal{F}$ satisfies the following properties:

(1) $\mathcal{F}(X \coprod Y) \cong \mathcal{F}(X) \oplus \mathcal{F}(Y)$.

(2) For any smooth affine curve $X / S$ and any effective Cartier divisor $D \subset X$ finite and surjective over $S$ we have a canonical homomorphism $\operatorname{Tr}_{D}: \mathcal{F}(X) \rightarrow \mathcal{F}(S)$. If $D \subset X$ is the graph of some section $i: S \rightarrow X$, then $\operatorname{Tr}_{D}=\mathcal{F}(i)$. Moreover these transfer homomorphisms are compatible with pull-backs.

(3) $\operatorname{Tr}_{D}+\operatorname{Tr}_{D^{\prime}}=\operatorname{Tr}_{D \cdot D^{\prime}}$ whenever the restriction of the line bundle $I_{D}$ to $D^{\prime}$ is trivial.

If in addition $\mathcal{F}\left(X \times \mathbb{A}^{1}\right)=\mathcal{F}(X)$ for any $X \in S m / F$, then we say that $\mathcal{F}$ is a homotopy invariant pseudo pretheory.

As always we extend canonically all functors defined on the category $S m / F$ to the wider category of appropriate pro-schemes (which includes at least all semilocalizations of all smooth schemes) by taking direct limits. The above properties obviously remain true after such extension as well.

We denote by $C_{0}(X / S)$ the free abelian group generated by closed integral subschemes $D \subset X$ finite and surjective over $S$. Let $c(D) \in C_{0}(X / S)$ denote the Weil divisor corresponding to the Cartier divisor $D$. We shall also consider the corresponding singular homology group

$$
H_{0}(X / S) \equiv C_{0}(X / S) /\left\langle\left\{D_{0}-D_{1}, D \in C_{0}\left(X \times \mathbb{A}^{1} / S \times \mathbb{A}^{1}\right)\right\}\right\rangle
$$

(cf. [30]).

Our first proposition verifies that the transfers on a pseudo pretheory $\mathcal{F}$ are sufficiently well behaved to give an action of relative 0 -cycles on $\mathcal{F}(S)$ for $S$ semilocal. 
Proposition 10.1. - Let $\mathcal{F}$ be a homotopy invariant pseudo pretheory and let $S / F$ be a smooth connected semilocal scheme. Let further $X / S$ be a smooth affine curve over $S$. Then there exists a natural pairing

$$
\mathcal{F}(X) \otimes C_{0}(X / S) \stackrel{\operatorname{Tr}}{\longrightarrow} \mathcal{F}(S)
$$

which is uniquely characterized by the formula $\operatorname{Tr}(f \otimes c(D))=\operatorname{Tr}_{D}(f)$ for any $f \in \mathcal{F}(X)$ and any effective Cartier divisor $D \subset X$ finite and surjective over $S$. Moreover this pairing factors through a pairing

$$
\mathcal{F}(X) \otimes H_{0}(X / S) \rightarrow \mathcal{F}(S) .
$$

Proof. - Observe that for the semilocal scheme $S$ the assumptions of (3) are always satisfied, since in this case the scheme $D^{\prime}$ is also semilocal and hence $\operatorname{Pic}\left(D^{\prime}\right)=0$. This shows that

$$
\operatorname{Tr}_{D \cdot D^{\prime}}=\operatorname{Tr}_{D}+\operatorname{Tr}_{D^{\prime}}: \mathcal{F}(X) \rightarrow \mathcal{F}(S) \quad \forall D, D^{\prime}
$$

Thus the pairing $(f, D) \mapsto \operatorname{Tr}_{D}(f)$ is bilinear and hence factors to define the pairing in question (since $C_{0}(X / S)$ is precisely the group completion of the abelian monoid of effective Cartier divisors finite and surjective over $S$ ). Assume now that $D \subset X \times \mathbb{A}^{1}$ is an effective Cartier divisor finite and surjective over $S \times \mathbb{A}^{1}$. Denote by $D_{0}, D_{1} \subset X$ the effective Cartier divisors (finite and surjective over $S$ ) obtained as pull-backs of $D$ under the two standard embeddings $i_{0}, i_{1}: S \hookrightarrow S \times \mathbb{A}^{1}$. Let $f \in \mathcal{F}(X)=\mathcal{F}\left(X \times \mathbb{A}^{1}\right)$ be an arbitrary element. The compatibility of transfers with pull-backs shows that the pull-backs of $\operatorname{Tr}_{D}(f) \in \mathcal{F}\left(S \times \mathbb{A}^{1}\right)=\mathcal{F}(S)$ under the embeddings $i_{0}$ and $i_{1}$ are equal to $\operatorname{Tr}_{D_{0}}(f)$ and $\operatorname{Tr}_{D_{1}}(f)$ respectively. In view of the homotopy invariance of the functor $\mathcal{F}$ these pull-backs are the same. Thus $\operatorname{Tr}_{D_{0}}(f)=\operatorname{Tr}_{D_{1}}(f)$, i.e., our pairing kills elements of the form $c\left(D_{0}\right)-c\left(D_{1}\right)$. Since such elements generate the kernel of the surjection $C_{0}(X / S) \rightarrow H_{0}(X / S)$, the statement follows.

The next proposition is the analogue of $[33,4.17]$ for pseudo pretheories.

Proposition 10.2. - Let $X \in S m / F$ be a smooth irreducible affine scheme over $F$ and let $x=\left\{x_{1}, \ldots, x_{n}\right\}$ be a finite number of points of $X$. Denote by $X_{x}$ the semilocalization of $X$ in $x$. Let finally $U \neq \emptyset$ be an open subscheme of $X$. Then for any homotopy invariant pseudo pretheory $\mathcal{F}$ there exists a homomorphism $\varphi: \mathcal{F}(U) \rightarrow \mathcal{F}\left(X_{x}\right)$ such that the following diagram commutes

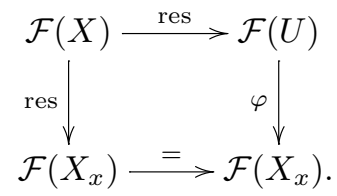

Proof. - The proof essentially repeats the proof of the Proposition 4.17 [33] so we only sketch the main points. The geometric part of the argument remains unchanged. We may obviously assume that all the points $x_{i}$ are closed, we note also that we can always diminish both $X$ and $U$ if necessary. Proposition 4.9 [33] shows that upon diminishing $X$ and $U$ we may assume that there exists a smooth affine morphism $p: X \rightarrow S$ of relative dimension one (i.e., $X$ is a smooth curve over a smooth affine $S$ ) whose restriction to $Z=X \backslash U$ is finite (cf. also [25] Lemma 5.12). Moreover we may assume that there exists a proper morphism of relative dimension one $\bar{p}: \bar{X} \rightarrow S$ with $\bar{X}$ normal and integral and an open embedding $i: X \hookrightarrow \bar{X}$ such that $\bar{p} \circ i=p$. Finally we may assume also that the following property holds (where we set 
$\left.X_{\infty}=\bar{X} \backslash X\right)$ : the closed subscheme $Z \coprod X_{\infty} \subset \bar{X}$ admits an open affine neighborhood (in other words $\bar{X}$ is a good compactification for both $X \rightarrow S$ and $U \rightarrow S$ - see [30] for the definition of the good compactification). Consider now a smooth affine curve $U \times{ }_{S} X_{x} \rightarrow X_{x}$. According to Proposition 10.1 each element $D \in H_{0}\left(U \times_{S} X_{x} / X_{x}\right)$ defines a homomorphism

$$
\mathcal{F}(U) \rightarrow \mathcal{F}\left(U \times_{S} X_{x}\right) \stackrel{\operatorname{Tr}_{D / X_{x}}}{\longrightarrow} \mathcal{F}\left(X_{x}\right),
$$

where the first map is given by the contravariant functoriality of $\mathcal{F}$. In the same way each element $D \in H_{0}\left(X \times_{S} X_{x} / X_{x}\right)$ defines a homomorphism $\mathcal{F}(X) \stackrel{\operatorname{Tr}_{D}}{\longrightarrow} \mathcal{F}\left(X_{x}\right)$ and the restriction homomorphism res: $\mathcal{F}(X) \rightarrow \mathcal{F}\left(X_{x}\right)$ coincides with $\operatorname{Tr}_{D_{0}}$ where $D_{0} \subset X \times_{S} X_{x} / X_{x}$ is the graph of the natural $S$-morphism $X_{x} \rightarrow X$. Thus the statement would follow if we can show that the homomorphism

$$
H_{0}\left(U \times_{S} X_{x} / X_{x}\right) \rightarrow H_{0}\left(X \times_{S} X_{x} / X_{x}\right)
$$

induced by the open embedding $U \hookrightarrow X$ is surjective. Let $\bar{Y}$ be the normalization of the disjoint sum of components of $\bar{X} \times{ }_{S} X_{x}$ considered as closed reduced subschemes. The relative curve $\bar{Y} \rightarrow X_{x}$ is easily seen to be a good compactification for both $U \times_{S} X_{x} \rightarrow X_{x}$ and $X \times_{S} X_{x} \rightarrow X_{x}$. Thus setting $Y_{\infty}=\bar{Y} \backslash X \times_{S} X_{x}$ we have the following computation of the corresponding $H_{0}$ 's:

$$
\begin{aligned}
& H_{0}\left(U \times{ }_{S} X_{x} / X_{x}\right)=\operatorname{Pic}\left(\bar{Y}, Y_{\infty} \coprod Z \times_{S} X_{x}\right), \\
& H_{0}\left(X \times{ }_{S} X_{x} / X_{x}\right)=\operatorname{Pic}\left(\bar{Y}, Y_{\infty}\right),
\end{aligned}
$$

see [30]. Finally we note that the scheme $Z \times_{S} X_{x}$ is semilocal and hence has trivial Picard group. The short exact sequence (cf. [30, (1)]

$$
\operatorname{Pic}\left(\bar{Y}, Y_{\infty} \coprod Z \times_{S} X_{x}\right) \rightarrow \operatorname{Pic}\left(\bar{Y}, Y_{\infty}\right) \rightarrow \operatorname{Pic}\left(Z \times_{S} X_{x}\right)=0
$$

concludes the proof.

The injectivity property we require is now an easy consequence.

THEOREM 10.3. - For any homotopy invariant pseudo pretheory $\mathcal{F}$ and any smooth affine irreducible semilocal scheme $S$, the restriction map $\mathcal{F}(S) \rightarrow \mathcal{F}(F(S))$ is injective, where $F(S)$ is the field of rational functions on $S$.

Proof. - Assume that $S$ is the semilocalization of a smooth affine scheme $X$ in the finite set of points $x=\left\{x_{1}, \ldots, x_{n}\right\}$. Since

$$
\mathcal{F}(S)=\lim _{x \subset U} \mathcal{F}(U), \quad \mathcal{F}(F(S))=\lim _{U \neq \emptyset} \mathcal{F}(U),
$$

it would suffice to show that if a certain element of $\mathcal{F}(X)$ dies being restricted to some non empty $U$ then it also dies being restricted to $X_{x}=S$. However this is obvious from Proposition 10.2.

COROLlARY 10.4. - Assume that the homotopy invariant pseudo pretheory $\mathcal{F}$ satisfies the condition that $\mathcal{F}(E)=0$ for any finitely generated separable extension $E / F$. Then $\mathcal{F}(S)=0$ for any smooth affine semilocal $S$. 


\section{Extension from fields to semi-local rings}

In Theorem 8.9, we exhibited homotopy fibration sequences

$$
\mathcal{K}^{\mathcal{Q}, \mathbb{A}^{q+1}}\left(\Delta^{\bullet}\right) \rightarrow \mathcal{K}^{\mathcal{Q}, \mathbb{A}^{q}}\left(\Delta^{\bullet}\right) \rightarrow \mathbb{B}\left(C \bullet\left(\mathbb{Z}^{\mathcal{Q}, \mathbb{A}^{q}}\right)(F)\right)
$$

which determine the first derived exact couple of the Bloch-Lichtenbaum exact couple and thus the spectral sequence (5.6.0). Since the composition of the maps is easily seen to be trivial, the fundamental result is the assertion that the induced map from the first space to the homotopy fibre of the second map is a weak equivalence. Our approach to extending this result is straightforward: for more general smooth schemes $S$ over $F$, consider the sequence of maps

$$
\mathcal{K}^{\mathcal{Q}, \mathbb{A}^{q+1}}\left(\Delta^{\bullet} \times_{F} S\right) \rightarrow \mathcal{K}^{\mathcal{Q}, \mathbb{A}^{q}}\left(\Delta^{\bullet} \times_{F} S\right) \rightarrow \mathbb{B}\left(C \bullet\left(\mathbb{Z}^{\mathcal{Q}, \mathbb{A}^{q}}\right)(S)\right)
$$

and show that the induced map from the first space to the homotopy fibre of the second map is a homotopy equivalence or equivalently that the kernel and cokernel of the induced maps on homotopy groups vanish.

In this section, we employ Voevodsky's technique extended to pseudo pretheories as formulated in Theorem 10.3 to obtain fibration sequences for smooth, affine semi-local schemes $S$. To carry out this argument, we must verify that the functors

$$
\begin{aligned}
& X \mapsto \pi_{i}\left(\mathcal{K}^{\mathcal{Q}, \mathbb{A}^{q}}\left(\Delta^{\bullet} \times X\right)\right), \\
& X \mapsto \pi_{i}\left(f i b\left(\mathcal{K}^{\mathcal{Q}, \mathbb{A}^{q}}\left(\Delta^{\bullet} \times X\right) \stackrel{\text { can }}{\longrightarrow} \mathbb{B}\left(C \bullet\left(\mathbb{Z}^{\mathcal{Q}, \mathbb{A}^{q}}\right)(X)\right)\right)\right)
\end{aligned}
$$

are indeed pseudo pretheories.

Let $S \in S m / F$ be a smooth scheme and let $X / S$ be a smooth affine curve over $S$. Let further $D \subset X$ be an effective Cartier divisor (i.e., a closed subscheme whose defining sheaf of ideals is a line bundle) on $X$, which is finite and surjective over $S$. Since $D$ is locally defined by a single equation, which is a non-zero divisor in the fiber, the projection $p_{D}: D \rightarrow S$ is also flat. Thus according to the results of Section 9 we see that for any $q \geqslant 0$ we get a natural morphism of simplicial prespectra

$$
\operatorname{Tr}_{D}: \mathcal{K}^{\mathcal{Q}, \mathbb{A}^{q}}\left(\Delta^{\bullet} \times X\right) \rightarrow \mathcal{K}^{\mathcal{Q}, \mathbb{A}^{q}}\left(\Delta^{\bullet} \times D\right) \stackrel{\operatorname{Tr}_{D / S}}{\longrightarrow} \mathcal{K}^{\mathcal{Q}, \mathbb{A}^{q}}\left(\Delta^{\bullet} \times S\right)
$$

Here the first arrow is the obvious restriction map whereas the second arrow is the transfer homomorphism (defined in Section 9) corresponding to the finite flat morphism $D \rightarrow S$. As was discussed in Section 9 the construction of $\operatorname{Tr}_{D / S}$ depends on certain choices; however the resulting morphism is well defined up to homotopy and in particular the corresponding homomorphisms in homotopy groups are independent of the choices made.

The etale sheaf $\mathbb{Z}^{\mathcal{Q}, \mathbb{A}^{q}}$, which is a presheaf with transfers as mentioned prior to Lemma 8.7, has transfer maps which are particularly easy to define in the context above: $\operatorname{Tr}_{D / S}$ is given by pull-back of cycles to $D$, then push-forward of cycles to $S$.

Recalling the construction of the homomorphism

$$
K_{0}^{\mathcal{Q}, \mathbb{A}^{q}}\left(\Delta^{n} \times X\right) \stackrel{\text { can }}{\longrightarrow} \mathbb{Z}^{\mathcal{Q}, \mathbb{A}^{q}}\left(\Delta^{n} \times X\right)
$$

in Lemma 8.7, we verify easily the following lemma.

LEMma 11.1. - As above, let $S \in S m / F$ be a smooth scheme, let $X / S$ be a smooth affine curve, and let $D$ be an effective Cartier divisor which is finite and surjective over $S$. 
(1) For any $n, q \geqslant 0$ the following diagram commutes

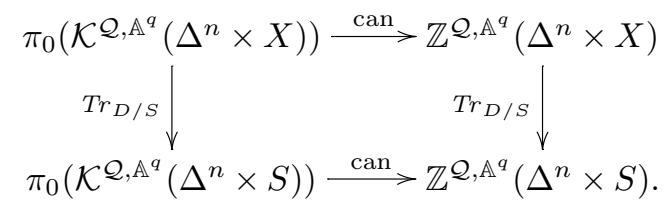

Here the left vertical arrow is the map in homotopy groups induced by the morphism of prespectra $\operatorname{Tr}_{D}: \mathcal{K}^{\mathcal{Q}, \mathbb{A}^{q}}\left(\Delta^{n} \times X\right) \rightarrow \mathcal{K}^{\mathcal{Q}, \mathbb{A}^{q}}\left(\Delta^{n} \times S\right)$ and the right vertical arrow is the transfer map corresponding to the presheaf with transfers $\mathbb{Z}^{\mathcal{Q}, \mathbb{A}^{q}}$.

(2) We have a commutative diagram of simplicial prespectra

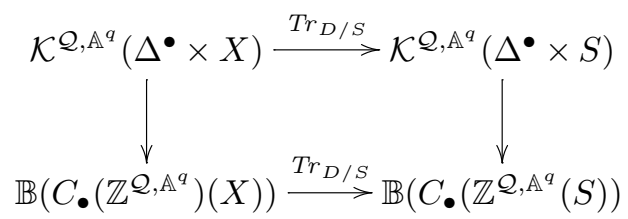

and hence the morphism $\operatorname{Tr}_{D / S}$ defines also a morphism of prespectra

$$
\begin{aligned}
& \operatorname{Tr}_{D / S}: f i b\left(\mathcal{K}^{\mathcal{Q}, \mathbb{A}^{q}}\left(\Delta^{\bullet} \times X\right) \stackrel{\text { can }}{\longrightarrow} \mathbb{B}\left(C \bullet\left(\mathbb{Z}^{\mathcal{Q}, \mathbb{A}^{q}}\right)(X)\right)\right) \\
& \quad \rightarrow f i b\left(\mathcal{K}^{\mathcal{Q}, \mathbb{A}^{q}}\left(\Delta^{\bullet} \times S\right) \stackrel{\text { can }}{\longrightarrow} \mathbb{B}\left(C \bullet\left(\mathbb{Z}^{\mathcal{Q}, \mathbb{A}^{q}}\right)(S)\right)\right) .
\end{aligned}
$$

(3) The morphism of prespectra

$$
\mathcal{K}^{\mathcal{Q}, \mathbb{A}^{q+1}}\left(\Delta^{\bullet} \times X\right) \rightarrow f i b\left(\mathcal{K}^{\mathcal{Q}, \mathbb{A}^{q}}\left(\Delta^{\bullet} \times X\right) \stackrel{\text { can }}{\longrightarrow} \mathbb{B}\left(C \bullet\left(\mathbb{Z}^{\mathcal{Q}, \mathbb{A}^{q}}\right)(X)\right)\right)
$$

is compatible with transfers.

The transfer maps defined by effective divisors finite and surjective over $S$ are compatible with pull-backs. The following result is an obvious corollary of Lemmas 9.3 and 11.1.

Proposition 11.2.- Let $f: S^{\prime} \rightarrow S$ be a morphism in $S m / F$. Consider the Cartesian diagram

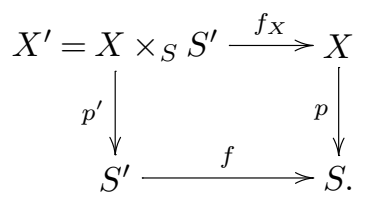

Let $D$ be an effective Cartier divisor on $X$ finite and surjective over $S$ and let further $D^{\prime}=D \times{ }_{S} S^{\prime}$ be the corresponding Cartier divisor on $X^{\prime}$. Then the following diagram commutes

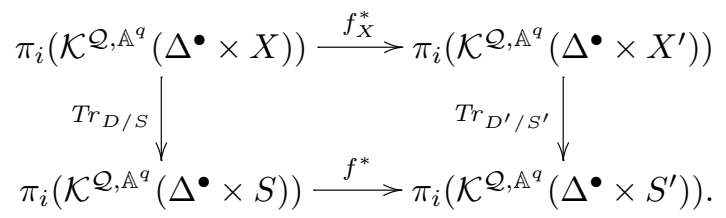


The same applies equally if we replace everywhere the prespectrum $\mathcal{K}^{\mathcal{Q}, \mathbb{A}^{q}}\left(\Delta^{\bullet} \times X\right)$ by the prespectrum $f i b\left(\mathcal{K}^{\mathcal{Q}, \mathbb{A}^{q}}\left(\Delta^{\bullet} \times X\right) \stackrel{\text { can }}{\longrightarrow} \mathbb{B}\left(C_{\bullet}\left(\mathbb{Z}^{\mathcal{Q}, \mathbb{A}^{q}}\right)(X)\right)\right)$. In other words transfer maps we have defined are compatible with pull-backs.

As mentioned at the beginning of Section $10, S \mapsto \pi_{i}\left(\mathcal{K}^{\mathcal{Q}, \mathbb{A}^{q}}\left(\Delta^{\bullet} \times S\right)\right)$ together with the transfer maps $\operatorname{Tr}_{D / S}$ is not a pretheory in the sense of Voevodsky [33]. Nevertheless, these transfers are sufficiently well behaved to determine the structure of pseudo pretheories.

Proposition 11.3. - Let $D$ and $D^{\prime}$ be effective Cartier divisors on $X$ which are finite and surjective over $S$. Assume further that the restriction of the sheaf of ideals $I_{D}$ (defining $D$ on $X$ ) to the scheme $D^{\prime}$ is a trivial line bundle. Then $\operatorname{Tr}_{D \cdot D^{\prime}}=\operatorname{Tr}_{D}+\operatorname{Tr}_{D^{\prime}}$.

Proof. - Denote by $i: D \hookrightarrow D \cdot D^{\prime}, i^{\prime}: D^{\prime} \hookrightarrow D \cdot D^{\prime}$ the corresponding closed embeddings. One checks easily that we have the following short exact sequence of coherent sheaves on $D \cdot D^{\prime}$

$$
0 \rightarrow i_{*}^{\prime}\left(I_{D} \otimes_{\mathcal{O}_{X}} \mathcal{O}_{D^{\prime}}\right) \rightarrow \mathcal{O}_{D \cdot D^{\prime}} \rightarrow i_{*}\left(\mathcal{O}_{D}\right) \rightarrow 0
$$

Let $f \in \Gamma\left(D^{\prime}, I_{D} \otimes_{\mathcal{O}_{X}} \mathcal{O}_{D^{\prime}}\right)$ be the trivialization of the line bundle $I_{D} \otimes_{\mathcal{O}_{X}} \mathcal{O}_{D^{\prime}}$. Multiplication by $f$ defines a short exact sequence of coherent $\mathcal{O}_{D \cdot D^{\prime}}$-modules

$$
0 \rightarrow i_{*}^{\prime}\left(\mathcal{O}_{D^{\prime}}\right) \stackrel{f}{\rightarrow} \mathcal{O}_{D \cdot D^{\prime}} \rightarrow i_{*}\left(\mathcal{O}_{D}\right) \rightarrow 0
$$

For any vector bundle $P \in \mathcal{P}(X)$ the above exact sequence gives the following exact sequence

$$
0 \rightarrow i_{*}^{\prime}\left(P \otimes_{\mathcal{O}_{X}} \mathcal{O}_{D^{\prime}}\right) \stackrel{f}{\rightarrow} P \otimes_{\mathcal{O}_{X}} \mathcal{O}_{D \cdot D^{\prime}} \rightarrow i_{*}\left(P \otimes_{\mathcal{O}_{X}} \mathcal{O}_{D}\right) \rightarrow 0
$$

Applying to the above exact sequence of coherent sheaves the exact functor $\left(p_{D \cdot D^{\prime}}\right)_{*}$ we get an exact sequence of vector bundles on $S$

$$
0 \rightarrow\left(p_{D^{\prime}}\right)_{*}\left(P \otimes_{\mathcal{O}_{X}} \mathcal{O}_{D^{\prime}}\right) \rightarrow\left(p_{D \cdot D^{\prime}}\right)_{*}\left(P \otimes_{\mathcal{O}_{X}} \mathcal{O}_{D \cdot D^{\prime}}\right) \rightarrow\left(p_{D}\right)_{*}\left(P \otimes_{\mathcal{O}_{X}} \mathcal{O}_{D}\right) \rightarrow 0 .
$$

Passing now from vector bundles on $X$ to the equivalent category of vector bundles on $S \mathrm{ch} / X$ we conclude immediately that multiplication by $f$ defines a homomorphism of functors (from $\mathcal{P}(S c h / X)$ to $\mathcal{P}(S c h / S)) f: \operatorname{Tr}_{D^{\prime}} \rightarrow \operatorname{Tr}_{D \cdot D^{\prime}}$ and moreover the following sequence of functors is exact

$$
0 \rightarrow \operatorname{Tr}_{D^{\prime}} \rightarrow \operatorname{Tr}_{D \cdot D^{\prime}} \rightarrow \operatorname{Tr}_{D} \rightarrow 0
$$

Now the statement follows easily from Waldhausen's Additivity Theorem [37].

COROLlaRY 11.4. - Let $\mathcal{F}$ denote either

$$
\begin{aligned}
& X \mapsto \pi_{i}\left(\mathcal{K}^{\mathcal{Q}, \mathbb{A}^{q}}\left(\Delta^{\bullet} \times X\right)\right), \\
& X \mapsto \pi_{i}\left(f i b\left(\mathcal{K}^{\mathcal{Q}, \mathbb{A}^{q}}\left(\Delta^{\bullet} \times X\right) \stackrel{\text { can }}{\longrightarrow} \mathbb{B}\left(C \bullet\left(\mathbb{Z}^{\mathcal{Q}, \mathbb{A}^{q}}\right)(X)\right)\right)\right) .
\end{aligned}
$$

Then $\mathcal{F}$ is a homotopy invariant pseudo pretheory (as discussed in Section 10).

Proof. - Homotopy invariance follows from Proposition 7.2, since both constructions are functorial on $S m / F$. The second and third defining properties of a pseudo pretheory are given by Propositions 11.2 and 11.3.

Combining Theorem 8.9, Theorem 10.3 and Corollary 11.4 , we now easily obtain the fibration sequences we seek for smooth, affine semilocal schemes. 
THEOREM 11.5. - Let S/F be a smooth, affine, semilocal scheme. Then the sequence of maps

$$
\mathcal{K}^{\mathcal{Q}, \mathbb{A}^{q+1}}\left(\Delta^{\bullet} \times_{F} S\right) \rightarrow \mathcal{K}^{\mathcal{Q}, \mathbb{A}^{q}}\left(\Delta^{\bullet} \times_{F} S\right) \rightarrow \mathbb{B}\left(C \bullet\left(\mathbb{Z}^{\mathcal{Q}, \mathbb{A}^{q}}\right)(S)\right)
$$

is a homotopy fibration sequence.

Proof. - We have to show that the induced homomorphisms

$$
\pi_{i}\left(\mathcal{K}^{\mathcal{Q}, \mathbb{A}^{q+1}}\left(\Delta^{\bullet} \times_{F} S\right)\right) \rightarrow \pi_{i}\left(f i b\left(\mathcal{K}^{\mathcal{Q}, \mathbb{A}^{q}}\left(\Delta^{\bullet} \times_{F} S\right) \rightarrow \mathbb{B}\left(C \bullet\left(\mathbb{Z}^{\mathcal{Q}, \mathbb{A}^{q}}\right)(S)\right)\right)\right)
$$

are isomorphisms for smooth affine semilocal $S$. By Corollary 11.4, the kernel and the cokernel of the above homomorphism of functors is a homotopy invariant pseudo pretheory. Since these pretheories vanish on fields according to Theorems 8.9 and 9.6, they vanish on all smooth affine semilocal schemes according to Corollary 10.4.

\section{Higher Chow groups and motivic cohomology}

In this section we fix our definition of motivic cohomology and show that motivic cohomology is naturally isomorphic to higher Chow groups of Bloch $[3,4]$.

Recall the etale sheaf with transfers $X \mapsto \mathbb{Z}^{\mathcal{Q}, \mathbb{A}^{q}}(X)$ introduced in Section 8: $\mathbb{Z}^{\mathcal{Q}, \mathbb{A}^{q}}(X)$ is the free abelian group on the closed integral subschemes $Z \subset X \times \mathbb{A}^{q}$ which are quasifinite and dominant over some connected component of $X$. Applying to this sheaf the singular complex construction (i.e., setting $C_{n}\left(\mathbb{Z}^{\mathcal{Q}, \mathbb{A}^{q}}\right)(X)=\mathbb{Z}^{\mathcal{Q}, \mathbb{A}^{q}}\left(\Delta^{n} \times X\right)$ ), we get a simplicial sheaf $C_{\bullet}\left(\mathbb{Z}^{\mathcal{Q}, \mathbb{A}^{q}}\right)$. We use the notation $C_{*}\left(\mathbb{Z}^{\mathcal{Q}, \mathbb{A}^{q}}\right)$ for the corresponding complex of sheaves with differential equal to the alternating sum of face operations. We set further $C^{i}\left(\mathbb{Z}^{\mathcal{Q}, \mathbb{A}^{q}}\right)=C_{-i}\left(\mathbb{Z}^{\mathcal{Q}, \mathbb{A}^{q}}\right)$, so that $C^{*}\left(\mathbb{Z}^{\mathcal{Q}, \mathbb{A}^{q}}\right)$ is a non positive complex of degree +1 . We define the motivic complex $\mathbb{Z}(q)$ as a degree shift of the complex $C^{*}\left(\mathbb{Z}^{\mathcal{Q}, \mathbb{A}^{q}}\right)$ :

$$
\mathbb{Z}(q)=C^{*}\left(\mathbb{Z}^{\mathcal{Q}, \mathbb{A}^{q}}\right)[-2 q]
$$

We define the motivic cohomology $H^{*}(X, \mathbb{Z}(q))$ of $X$ to be the (Zariski) hypercohomology of $X$ with coefficients in this complex of sheaves.

The fact that this definition agrees with the definition given in [34] is proved in [10] whenever $F$ admits resolution of singularities and is proved in [35] for general fields $F$. It has the advantage that the corresponding motivic cohomology groups always coincide with the higher Chow groups.

PROPOSITION 12.1. - For any smooth scheme $X \in S m / F$ there is a natural isomorphism in the derived category of complexes of sheaves on the small Zariski site of $X$

$$
C_{*}\left(\mathbb{Z}^{\mathcal{Q}, \mathbb{A}^{q}}\right) \cong z^{q}(-, *),
$$

where $z^{q}(U, n)$ is the free abelian group generated by closed integral subschemes $Z \subset \Delta^{n} \times U$ of codimension $q$ which intersect all faces of $\Delta^{n} \times U$ properly.

Proof. - Consider the following complex of sheaves on $X_{Z a r}$,

$$
U \mapsto z^{q}\left(U \times \mathbb{A}^{q}, *\right) .
$$


The homotopy invariance of higher Chow groups implies that the natural homomorphism

$$
z^{q}(-, *) \rightarrow z^{q}\left(-\times \mathbb{A}^{q}, *\right)
$$

is a quasi-isomorphism. Furthermore we have an obvious embedding of complexes

$$
C_{*}\left(\mathbb{Z}^{\mathcal{Q}, \mathbb{A}^{q}}\right) \hookrightarrow z^{q}\left(-\times \mathbb{A}^{q}, *\right) .
$$

The homomorphisms in homology induced by this embedding

$$
H_{n}\left(\mathbb{Z}^{\mathcal{Q}, \mathbb{A}^{q}}(X)\right) \rightarrow H_{n}\left(z^{q}\left(X \times \mathbb{A}^{q}, *\right)\right)=C H^{q}(X, n)
$$

are isomorphisms in the case of fields according to [29]. Note further that both sides are homotopy invariant presheaves with transfers. In the case of $H_{n}\left(\mathbb{Z}^{\mathcal{Q}, \mathbb{A}^{q}}(X)\right)$ this is obvious and in the case of the higher Chow groups this is verified in [36]. Since the displayed homomorphisms are obviously compatible with transfers, we further conclude from [33, 4.20] that these homomorphisms are isomorphisms for any smooth local scheme $X$, which shows that the embedding

$$
C_{*}\left(\mathbb{Z}^{\mathcal{Q}, \mathbb{A}^{q}}\right) \hookrightarrow z^{q}\left(-\times \mathbb{A}^{q}, *\right)
$$

is a quasi-isomorphism.

COROLLARY 12.2. - For any smooth quasiprojective scheme $X \in S m / F$ we have natural isomorphisms

$$
H^{p}(X, \mathbb{Z}(q)) \cong C H^{q}(X, 2 q-p) .
$$

Proof. - It suffices to note that higher Chow groups satisfy localization [4] and hence Zariski descent (i.e., are determined locally in the Zariski topology; cf. Proposition 13.2 below). This easily implies that the natural map from $C H^{q}(X,-n)$ to the $n$th hypercohomology group of the complex $z^{q}(-, *)$ (re-indexed cohomologically) is an isomorphism.

The symmetric group $\Sigma_{q}$ acts canonically on the scheme $\mathbb{A}^{q}$ which defines a (right) action of $\Sigma_{q}$ on the complex of sheaves $C_{*}\left(\mathbb{Z}^{\mathcal{Q}, \mathbb{A}^{q}}\right)$ and hence also an action of $\Sigma_{q}$ in motivic cohomology. The next result will be needed in Section 14.

LEMMA 12.3. - The natural action of $\Sigma_{q}$ in $H^{p}(X, \mathbb{Z}(q))$ is trivial.

Proof. - This follows immediately from the fact that both quasi-isomorphisms in the diagram

$$
z^{q}(-, *) \hookrightarrow z^{q}\left(-\times \mathbb{A}^{q}, *\right) \hookleftarrow C_{*}\left(\mathbb{Z}^{\mathcal{Q}, \mathbb{A}^{q}}\right)
$$

are $\Sigma_{q}$-equivariant, where the action of $\Sigma_{q}$ on $z^{q}(-, *)$ is trivial and that on $z^{q}\left(-\times \mathbb{A}^{q}, *\right)$ is induced by the natural action on $\mathbb{A}^{q}$.

We conclude this section by a discussion of products in motivic cohomology. This discussion is quite similar to that found in [39, 3.2] which uses somewhat different conventions. For any schemes $X, X^{\prime} \in S m / F$, by taking the direct product of cycles we get a natural map (cf. [10, $\S 8])$

$$
\mathbb{Z}^{\mathcal{Q}, \mathbb{A}^{q}}(X) \otimes \mathbb{Z}^{\mathcal{Q}, \mathbb{A}^{q^{\prime}}}\left(X^{\prime}\right) \rightarrow \mathbb{Z}^{\mathcal{Q}, \mathbb{A}^{q+q^{\prime}}}\left(X \times X^{\prime}\right)
$$


Applying this product operation to the schemes $X \times \Delta^{n}$ and $X^{\prime} \times \Delta^{m}$, we get a natural homomorphism of bisimplicial abelian groups

$$
\mathbb{Z}^{\mathcal{Q}, \mathbb{A}^{q}}\left(X \times \Delta^{\bullet}\right) \otimes \mathbb{Z}^{\mathcal{Q}, \mathbb{A}^{q^{\prime}}}\left(X^{\prime} \times \Delta^{\bullet}\right) \rightarrow \mathbb{Z}^{\mathcal{Q}, \mathbb{A}^{q+q^{\prime}}}\left(X \times X^{\prime} \times \Delta^{\bullet} \times \Delta^{\bullet}\right) .
$$

Composing this external product operation with the homomorphism induced by

$$
\Delta_{X}: X \rightarrow X \times X
$$

we get in the usual way for any $X \in S m / F$ a natural homomorphism of bisimplicial abelian groups

$$
\mathbb{Z}^{\mathcal{Q}, \mathbb{A}^{q}}\left(X \times \Delta^{\bullet}\right) \otimes \mathbb{Z}^{\mathcal{Q}, \mathbb{A}^{q^{\prime}}}\left(X \times \Delta^{\bullet}\right) \rightarrow \mathbb{Z}^{\mathcal{Q}, \mathbb{A}^{q+q^{\prime}}}\left(X \times \Delta^{\bullet} \times \Delta^{\bullet}\right) .
$$

Consider now the homomorphism of the associated total complexes and utilize the fact that the complex $\operatorname{Tot}\left(\mathbb{Z}^{\mathcal{Q}, \mathbb{A}^{q+q^{\prime}}}\left(X \times \Delta^{\bullet} \times \Delta^{\bullet}\right)\right)$ is canonically homotopy equivalent to the complex $\mathbb{Z}^{\mathcal{Q}, \mathbb{A}^{q+q^{\prime}}}\left(X \times \Delta^{\bullet}\right)$ (see [31] §0). Explicitly, the homomorphism

$$
\mathbb{Z}^{\mathcal{Q}, \mathbb{A}^{q+q^{\prime}}}\left(X \times \Delta^{\bullet}\right) \rightarrow \operatorname{Tot}\left(\mathbb{Z}^{\mathcal{Q}, \mathbb{A}^{q+q^{\prime}}}\left(X \times \Delta^{\bullet} \times \Delta^{\bullet}\right)\right)
$$

is induced by the projection onto the first copy of $\Delta^{\bullet}$, whereas the homotopy inverse map is defined by the shuffle map

$$
\mathbb{Z}^{\mathcal{Q}, \mathbb{A}^{q+q^{\prime}}}\left(X \times \Delta^{n} \times \Delta^{m}\right) \stackrel{\sum_{\phi:[n+m] \rightarrow[n] \times[m]} \varepsilon(\phi) \phi^{*}}{\longrightarrow} \mathbb{Z}^{\mathcal{Q}, \mathbb{A}^{q+q^{\prime}}}\left(X \times \Delta^{n+m}\right) .
$$

Here the sum is taken over all strictly increasing maps $\phi:[n+m] \rightarrow[n] \times[m]$ (which are in one to one correspondence with $(n, m)$ shuffles), $\varepsilon(\phi)$ denotes the sign of the corresponding shuffle and we use the same letter $\phi$ to denote the linear isomorphism of schemes $\Delta^{n+m} \rightarrow \Delta^{n} \times \Delta^{m}$ which coincides with $\phi$ on the set of vertices.

The previous considerations imply that we have a canonical pairing of complexes of sheaves

$$
\mathbb{Z}^{\mathcal{Q}, \mathbb{A}^{q}}\left(X \times \Delta^{\bullet}\right) \otimes \mathbb{Z}^{\mathcal{Q}, \mathbb{A}^{q^{\prime}}}\left(X \times \Delta^{\bullet}\right) \rightarrow \mathbb{Z}^{\mathcal{Q}, \mathbb{A}^{q+q^{\prime}}}\left(X \times \Delta^{\bullet}\right)
$$

explicitly given by the formula

$$
\left(Z, Z^{\prime}\right) \mapsto \sum_{\phi=\left(\phi^{\prime}, \phi^{\prime \prime}\right):[n+m] \rightarrow[n] \times[m]} \varepsilon(\phi)\left\{\left(\phi^{\prime}\right)^{*}(Z) \times_{X \times \Delta^{n+m}}\left(\phi^{\prime \prime}\right)^{*}\left(Z^{\prime}\right)\right\}
$$

(cf. [39, 2.3]). Re-indexing, we get a pairing of complexes

$$
\mathbb{Z}(q) \otimes \mathbb{Z}\left(q^{\prime}\right) \rightarrow \mathbb{Z}\left(q+q^{\prime}\right) .
$$

As discussed in Appendix A, this defines natural pairings in the hypercohomology groups

$$
H^{p}(X, \mathbb{Z}(q)) \otimes H^{p^{\prime}}\left(X, \mathbb{Z}\left(q^{\prime}\right)\right) \rightarrow H^{p+p^{\prime}}\left(X, \mathbb{Z}\left(q+q^{\prime}\right)\right) .
$$

The following properties of the product structure in motivic cohomology are straightforward from the definitions and Lemma 12.3 . 
LEMMA 12.4. - The bigraded ring $H^{*}(X, \mathbb{Z}(*))$ is associative and graded commutative with respect to the cohomological index.

Remark 12.5. - As shown in [39], if resolution of singularities holds, then the above product structure coincides with the one introduced in [34,31].

\section{The global spectral sequence}

In this section, we complete the program described at the beginning of Section 11 to produce homotopy fibration sequences and thus the globalization of the spectral sequence (5.6.0). As we have seen, the task is to show that the kernel and cokernel of certain natural maps of homotopy groups vanish. In Section 11, we verified this for smooth, affine semilocal schemes $S$. To globalize this result, we employ the techniques of simplicial sheaves established by Brown and Gersten [9] which are recalled in Appendix D.

For any $X \in S m / F$ we denote by $\Omega^{-1} \mathcal{K}^{q}$ the simplicial sheaf on $X$ associated to the presheaf $\left(U \mapsto S_{\bullet}^{\mathcal{Q}, \mathbb{A}^{q}}\left(\Delta^{\bullet} \times U\right) \equiv w S_{\bullet}\left(\mathcal{C P}^{\mathcal{Q}}\left(\Delta^{\bullet} \times U \times \mathbb{A}\right)\right)\right)$ :

$$
\Omega^{-1} \mathcal{K}^{q}:\left(U \mapsto S_{\bullet}^{\mathcal{Q}, \mathbb{A}^{q}}\left(\Delta^{\bullet} \times U\right)\right)_{Z a r} .
$$

For every $q \geqslant 0$ we have a sequence of simplicial sheaves

$$
\Omega^{-1} \mathcal{K}^{q+1} \rightarrow \Omega^{-1} \mathcal{K}^{q} \rightarrow B\left(C \bullet\left(\mathbb{Z}^{\mathcal{Q}, \mathbb{A}^{q}}\right)\right)
$$

and Theorem 11.5 implies that the conditions of Proposition D.5 are satisfied.

Thus we obtain the following consequence of Lemma D.7.

Proposition 13.1. - For any $X \in S m / F$ and any $q \geqslant 0$, we have a homotopy fibration sequence

$$
R \Gamma\left(X, \Omega^{-1} \mathcal{K}^{q+1}\right) \rightarrow R \Gamma\left(X, \Omega^{-1} \mathcal{K}^{q}\right) \rightarrow R \Gamma\left(X, B\left(C \bullet\left(\mathbb{Z}^{\mathcal{Q}, \mathbb{A}^{q}}\right)\right)\right)
$$

and hence a long exact homotopy sequence

$$
\begin{aligned}
& \rightarrow \pi_{n}\left(R \Gamma\left(X, \Omega^{-1} \mathcal{K}^{q}\right)\right) \rightarrow \pi_{n}\left(R \Gamma\left(X, B C \bullet\left(\mathbb{Z}^{\mathcal{Q}, \mathbb{A}^{q}}\right)\right)\right) \stackrel{\partial}{\rightarrow} \pi_{n-1}\left(R \Gamma\left(X, \Omega^{-1} \mathcal{K}^{q+1}\right)\right) \\
& \rightarrow \cdots \rightarrow \pi_{0}\left(R \Gamma\left(X, \Omega^{-1} \mathcal{K}^{q}\right)\right) \rightarrow \pi_{0}\left(R \Gamma\left(X, B C \bullet\left(\mathbb{Z}^{\mathcal{Q}, \mathbb{A}^{q}}\right)\right)\right) .
\end{aligned}
$$

Proposition 13.1 gives us a tower of homotopy fibration sequences

$$
\begin{gathered}
R \Gamma\left(X, \Omega^{-1} \mathcal{K}^{q+1}\right) \longrightarrow R \Gamma\left(X, \Omega^{-1} \mathcal{K}^{q}\right) \longrightarrow \\
\downarrow \\
R \Gamma\left(X, B\left(C \bullet\left(\mathbb{Z}^{\mathcal{Q}, \mathbb{A}^{q}}\right)\right)\right)
\end{gathered}
$$

Our goal is to show that this tower satisfies the conditions of Proposition 6.1 and hence defines a strongly convergent spectral sequence with limit $K_{*}(X)$.

Proposition 13.2. - For $X \in S m / F, \pi_{n}\left(R \Gamma\left(X, \Omega^{-1} \mathcal{K}^{0}\right)\right)=K_{n-1}(X)$.

Proof.-Since algebraic $K$-theory is homotopy invariant, the bisimplicial presheaf $U \mapsto S_{\bullet}^{\mathcal{Q}, \mathbb{A}^{0}}\left(\Delta^{\bullet} \times U\right)=w S_{\bullet}\left(\mathcal{C P}\left(\Delta^{\bullet} \times U\right)\right)$ is pointwise weakly equivalent to the simplicial presheaf $U \mapsto w S_{\bullet}(\mathcal{C P}(U))$. Moreover, $w S_{\bullet}(\mathcal{C P}(U))$ is the first delooping of the space whose 
homotopy groups are the $K$-groups of $U$. As seen in [9, Theorem 4], the Mayer-Vietoris property for algebraic K-theory implies that the canonical morphism

$$
\Gamma\left(X, w S_{\bullet}(\mathcal{C P}(-))\right) \rightarrow R \Gamma\left(X, w S_{\bullet}(\mathcal{C P}(-))_{Z a r}\right)
$$

is a weak equivalence.

LEMMA 13.3. - Let $I_{\bullet}$ be a simplicial abelian sheaf on $X$ and let $M_{*}$ denote the non-negative Moore complex of sheaves on X corresponding to $I_{\bullet}(c f .(1.1 .1))$. The following conditions are equivalent

(1) The simplicial sheaf $I_{\bullet}$ is flasque.

(2) All terms of the complex $M_{*}$ except possibly for $M_{0}$ are flasque sheaves.

Proof. - The simplicial sheaf $I_{\bullet}$ is flasque if and only if for any open $V \subset U$ the homomorphism of simplicial abelian groups

$$
I_{\bullet}(U) \rightarrow I_{\bullet}(V)
$$

is a Kan fibration. However a homomorphism of simplicial abelian groups is a Kan fibration if and only if the corresponding homomorphism of non-negative complexes is surjective in positive degrees (see $[38,8.2 .5]$ ). Thus $I_{\bullet}$ is flasque if and only if for any $V \subset U$ and any $i>0$ the restriction homomorphism $M_{i}(U) \rightarrow M_{i}(V)$ is surjective, i.e., if and only if the sheaves $M_{i}(i>0)$ are flasque.

COROLlary 13.3.1. - Let $C_{\bullet}$ be a simplicial abelian sheafon $X$. Denote by $C_{*}$ (respectively by $\mathcal{M}_{*}$ ) the complex of abelian sheaves with terms $C_{i}$ and differential equal to the alternating sum of face operations (respectively the Moore complex corresponding to $C_{\bullet}$ ) and set $C^{i}=C_{-i}$, $\mathcal{M}^{i}=\mathcal{M}_{-i}$ so that $C^{*}$ (respectively $\mathcal{M}^{*}$ ) is a non-positive complex of degree +1 . Then for all $p \geqslant 0$ we have canonical isomorphisms

$$
H^{-p}\left(X, C_{\bullet}\right)=\pi_{p}\left(R \Gamma\left(X, C_{\bullet}\right)\right) \stackrel{\sim}{\longrightarrow} H^{-p}\left(X, \mathcal{M}^{*}\right)=H^{-p}\left(X, C^{*}\right) .
$$

Proof (cf. [9] Prop. 2). - The equality $H^{-p}\left(X, C^{*}\right)=H^{-p}\left(X, \mathcal{M}^{*}\right)$ follows from Corollary A.2. Since the functor $\mathcal{R}$ of Proposition D.6 commutes with products, we conclude immediately that the flasque simplicial sheaf $\mathcal{R}\left(C_{\bullet}\right)$ is actually a simplicial abelian sheaf. Moreover the natural weak equivalence $C_{\bullet} \stackrel{\phi}{\rightarrow} \mathcal{R}\left(C_{\bullet}\right)$ is a homomorphism of simplicial abelian sheaves. The corresponding homomorphism of non-positive complexes $\mathcal{M}^{*} \rightarrow \mathcal{M}\left(\mathcal{R}\left(C_{\bullet}\right)\right)^{*}$ is a quasiisomorphism and hence defines canonical homomorphisms

$$
\pi_{p}\left(R \Gamma\left(X, C_{\bullet}\right)\right)=H^{-p}\left(\Gamma\left(X, \mathcal{M}\left(\mathcal{R}\left(C_{\bullet}\right)\right)^{*}\right)\right) \rightarrow H^{-p}\left(X, \mathcal{M}^{*}\right),
$$

see Appendix A. Finally these homomorphisms are isomorphisms for $p \geqslant 0$ according to Corollary A.4, since all terms of the complex $\mathcal{M}\left(\mathcal{R}\left(C_{\bullet}\right)\right)^{*}$ in negative degrees are flasque.

Lemma 13.4. - Let $C_{\bullet}$ be a simplicial abelian sheaf and let $B C \bullet$ denote the classifying simplicial abelian sheaf for $C_{\bullet}$ ( $(c f$. following Proposition B.1). Then the associated complex $\left(B C_{\bullet}\right)_{*}$ is naturally quasi-isomorphic to the complex $C_{*}[1]$. Hence, passing to the cohomological notation, we get a canonical quasi-isomorphism $\left(B C_{\bullet}\right)^{*} \cong C^{*}[1]$ and induced isomorphisms in hypercohomology groups

$$
H^{p}\left(X,\left(B C_{\bullet}\right)^{*}\right)=H^{p+1}\left(X, C^{*}\right)
$$


Proof. - This follows immediately from Lemma B.3.

COROLLARY 13.4.1. - The homotopy groups of the space $R \Gamma\left(X, B C \bullet\left(\mathbb{Z}^{\mathcal{Q}, \mathbb{A}^{q}}\right)\right)$ are given by the formulas:

$$
\pi_{p}\left(R \Gamma\left(X, B C \cdot\left(\mathbb{Z}^{\mathcal{Q}, \mathbb{A}^{q}}\right)\right)\right) \cong H^{-p+2 q+1}(X, \mathbb{Z}(q))=C H^{q}(X, p-1)
$$

Thus, in particular this space is connected and its fundamental group is abelian.

Proof. - This follows immediately from Corollary 13.3.1 and Lemma 13.4.

LEMMA 13.5. - The space $R \Gamma\left(X, \Omega^{-1} \mathcal{K}^{q}\right)$ is $(q-\operatorname{dim} X)$-connected.

Proof. - Denote by $\pi_{n}^{q}$ Zariski sheaf associated to the presheaf

$$
U \mapsto \pi_{n}\left(\Omega^{-1} \mathcal{K}^{q}(U)\right)
$$

By definition, the stalk of $\pi_{n}^{q}$ at the point $x \in X$ coincides with

$$
\pi_{n-1}\left(\mathcal{K}^{\mathcal{Q}, \mathbb{A}^{q}}\left(\Delta^{\bullet} \times_{F} S\right)\right)
$$

where $S$ is the localization of $X$ at $x$. Theorem 10.3 and Corollary 11.4 show that this group injects into

$$
\pi_{n-1}\left(\mathcal{K}^{\mathcal{Q}, \mathbb{A}^{q}}\left(\Delta^{\bullet} \times{ }_{F} F(X)\right)\right) \cong \pi_{n-1}\left(\mathcal{K}^{\mathcal{C}^{q}}\left(\Delta^{\bullet} \times_{F} F(X)\right)\right) .
$$

Note further that the prespectrum $\mathcal{K}^{\mathcal{C}^{q}}\left(\Delta^{n} \times_{F} F(X)\right)$ is trivial provided that $q>n$, which implies that the prespectrum $\mathcal{K}^{\mathcal{F}^{q}}\left(\Delta^{\bullet} \times_{F} F(X)\right)$ is $(q-1)$-connected (see B.1). Thus the sheaf $\pi_{n}^{q}$ is trivial provided that $n \leqslant q$. Our statement is trivial in case $q<\operatorname{dim} X$. Thus we may assume that $q \geqslant \operatorname{dim} X$. In this case, $H^{i}\left(X, \pi_{n}^{q}\right)=0$ for $i \geqslant n$ which allows us to use the spectral sequence

$$
E_{2}^{i, j}=H^{i}\left(X, \pi_{j}^{q}\right) \Longrightarrow \pi_{j-i}\left(R \Gamma\left(X, \Omega^{-1} \mathcal{K}^{q}\right)\right)
$$

(see [9, Theorem 3]). This spectral sequence implies immediately that $\pi_{m}\left(R \Gamma\left(X, \Omega^{-1} \mathcal{K}^{q}\right)\right)=0$ for $m \leqslant q-\operatorname{dim} X$ (since $E_{2}^{i, j} \neq 0$ implies that $\left.j>q, i \leqslant \operatorname{dim} X\right)$.

Corollary 13.5.1. - The space $R \Gamma\left(X, \Omega^{-1} \mathcal{K}^{q}\right)$ is connected for all $q \geqslant 0$. Moreover the group $\pi_{1}\left(R \Gamma\left(X, \Omega^{-1} \mathcal{K}^{q}\right)\right)$ is abelian.

Proof. - We prove the first statement using decreasing induction on $q$. For $q \geqslant \operatorname{dim} X$, our first statement follows from Lemma 13.5. To pass from $q+1$ to $q$, it suffices to use the exact sequence of Proposition 13.1 together with Lemma 13.4.1. The second statement follows from the fact that that the space $\left|R \Gamma\left(X, \Omega^{-1} \mathcal{K}^{q}\right)\right|$ is an infinite loop space; this is verified in Proposition 13.10.

The results established above together with Proposition 6.1 prove the following Main Theorem.

THEOREM 13.6. - The tower of spaces

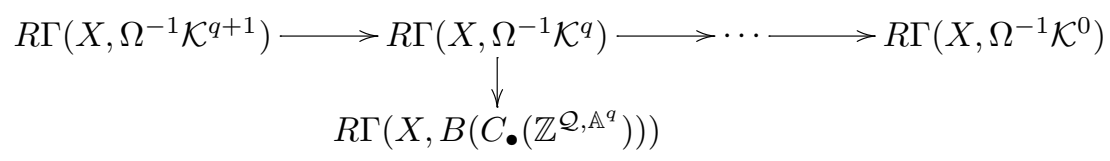


yields a strongly convergent spectral sequence

$$
E_{2}^{p, q}=H^{p-q}(X, \mathbb{Z}(-q))=C H^{-q}(X,-p-q) \Rightarrow K_{-p-q}(X) .
$$

In Section 15, we will need to know that the same spectral sequence may be obtained using the delooping presheaves $U \mapsto S_{\bullet} \ldots S_{\bullet}^{\mathcal{Q}, \mathbb{A}^{q}}\left(\Delta^{\bullet} \times U\right)$. We proceed to establish this result (in Proposition 13.10 below).

For any pointed simplicial set $K$, let $\mathcal{P}(K)$ denote the simplicial based path space of $K$, so that $p$-simplices of $\mathcal{P}(K)$ are maps of simplicial sets $\Delta^{p} \times \mathrm{I} \rightarrow K$ which take $\Delta^{p} \times 0$ to the distinguished point of $K$. Evaluation at 1 gives a canonical map $\mathcal{P}(K) \stackrel{\varepsilon_{1}}{\longrightarrow} K$. The fiber of $\varepsilon_{1}$ over the distinguished point of $K$ is the simplicial loop space $\Omega K$ of $K$. Thus $p$-simplices of $\Omega K$ are maps of simplicial sets $\Delta^{p} \times \mathrm{I} \rightarrow K$ which take $\Delta^{p} \times\{0,1\}$ to the distinguished point of $K$. The functor $\Omega$ (as in the case of topological spaces) is right adjoint to the functor $\Sigma$ : for any pointed simplicial set $L$ the set of pointed maps $L \rightarrow \Omega K$ is in one to one correspondence with the set of pointed maps $\Sigma L \rightarrow K$. Note further that for any $K$ we have canonical maps $|\mathcal{P} K| \rightarrow \mathcal{P}(|K|),|\Omega K| \rightarrow \Omega|K|$ and the following diagram commutes

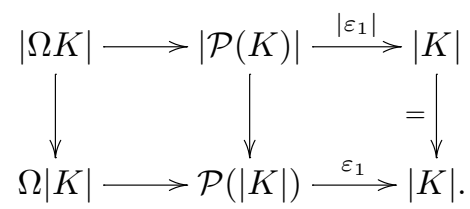

We skip the proof of the following elementary lemma.

LEMMA 13.7. - Let $K \rightarrow L$ be a Kan fibration of pointed simplicial sets. Then the obvious map $\mathcal{P}(K) \rightarrow \mathcal{P}(L) \times{ }_{L} K$ is again a Kan fibration and hence the induced map $\Omega(K) \rightarrow \Omega(L)$ is also a Kan fibration. In particular, for any Kan complex $K$ the map $\mathcal{P}(K) \stackrel{\varepsilon_{1}}{\longrightarrow} K$ is a Kan fibration and hence $\Omega(K)$ is also a Kan complex.

Since the space $|\mathcal{P}(K)|$ is contractible for any $K$ one concludes easily from Lemma 13.7 that for a Kan complex $K$ the natural map $|\Omega K| \rightarrow \Omega|K|$ is a weak equivalence.

For a pointed simplicial sheaf $K$ set

$$
(\mathcal{P}(K))(U)=\mathcal{P}(K(U)), \quad(\Omega(K))(U)=\Omega(K(U)) .
$$

One checks easily that $\mathcal{P}(K)$ and $\Omega(K)$ are again pointed simplicial sheaves. Moreover Lemma 13.7 implies that for a flasque simplicial sheaf $K$ the canonical morphism of simplicial sheaves

$$
\mathcal{P}(K) \stackrel{\varepsilon_{1}}{\longrightarrow} K
$$

is a global fibration and hence that the simplicial sheaves $\mathcal{P}(K)$ and $\Omega(K)$ are also flasque.

Let $K, K^{\prime}$ be pointed simplicial sheaves. Assume we are given a morphism $\Sigma K \stackrel{f}{\rightarrow} K^{\prime}$ such that for any $x \in X$ the induced map of topological spaces $\left|K_{x}\right| \rightarrow \Omega\left|K_{x}^{\prime}\right|$ is a weak equivalence. The morphism $\Sigma K=\mathrm{I} \times K /(\{0,1\} \times K \cup \mathrm{I} \times *) \rightarrow K^{\prime}$ defines a morphism

$$
\mathrm{I} \times \mathcal{R}(K) \rightarrow \mathcal{R}(\mathrm{I}) \times \mathcal{R}(K)=\mathcal{R}(\mathrm{I} \times K) \rightarrow \mathcal{R}\left(K^{\prime}\right)
$$

(cf. Proposition D.6). The naturality of $\mathcal{R}$ implies readily that the above morphism factors through $\Sigma \mathcal{R}(K)$. Thus we get canonical morphisms of simplicial sheaves $\Sigma \mathcal{R}(K) \rightarrow \mathcal{R}\left(K^{\prime}\right)$ 
and of simplicial sets of sections over $U \subset X$

$$
\Sigma R \Gamma(U, K) \rightarrow R \Gamma\left(U, K^{\prime}\right)
$$

LEMMA 13.8. - With the above notations and assumptions, the induced maps of topological spaces

$$
|R \Gamma(U, K)| \rightarrow\left|\Omega\left(R \Gamma\left(U, K^{\prime}\right)\right)\right| \rightarrow \Omega\left|R \Gamma\left(U, K^{\prime}\right)\right|
$$

are weak equivalences for any open $U \subset X$.

Proof. - Consider the commutative diagram

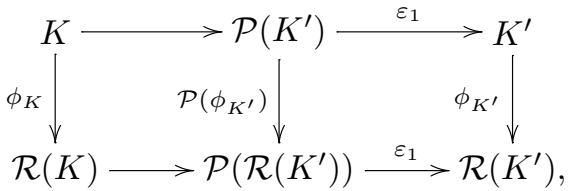

where $K \rightarrow \Omega\left(K^{\prime}\right) \subset \mathcal{P}\left(K^{\prime}\right)$ is adjoint to the given $\Sigma K \stackrel{f}{\rightarrow} K^{\prime}$. The top row satisfies the conditions of Proposition D.5 and the vertical arrows are weak equivalences. This implies that the bottom row satisfies conditions of Proposition D.5 as well. Since all simplicial sheaves appearing in the bottom row are flasque we conclude from Corollary D.5.1 that the sequence of simplicial sets

$$
R \Gamma(U, K) \rightarrow \Gamma\left(U, \mathcal{P}\left(\mathcal{R}\left(K^{\prime}\right)\right)\right)=\mathcal{P}\left(R \Gamma\left(U, K^{\prime}\right)\right) \rightarrow R \Gamma\left(U, K^{\prime}\right)
$$

is a homotopy fibration sequence and hence the map $|R \Gamma(U, K)| \rightarrow\left|\Omega\left(R \Gamma\left(U, K^{\prime}\right)\right)\right|$ is a weak equivalence. Finally the map $\left|\Omega\left(R \Gamma\left(U, K^{\prime}\right)\right)\right| \rightarrow \Omega\left|R \Gamma\left(U, K^{\prime}\right)\right|$ is also a weak equivalence since the simplicial set $R \Gamma\left(U, K^{\prime}\right)$ is a Kan complex.

Assume we are given a prespectrum of simplicial sheaves, i.e. a sequence of pointed simplicial sheaves $L^{q}$ together with pointed maps $\Sigma L^{q} \rightarrow L^{q+1}$. Applying to this sequence the functor $\mathcal{R}$, we get a sequence $\mathcal{R}\left(L^{q}\right)$ of fibrant simplicial sheaves and the associated sequence of sections over an open $U \subset X$

$$
R \Gamma\left(U, L^{q}\right)=\Gamma\left(U, \mathcal{R}\left(L^{q}\right)\right) .
$$

Moreover, as we saw above, the structure morphism $\Sigma L^{q} \rightarrow L^{q+1}$ defines canonical morphisms of simplicial sheaves $\Sigma \mathcal{R}\left(L^{q}\right) \rightarrow \mathcal{R}\left(L^{q+1}\right)$ and of simplicial sets of sections over $U \subset X$

$$
\Sigma R \Gamma\left(U, L^{q}\right) \rightarrow R \Gamma\left(U, L^{q+1}\right) .
$$

In other words $R \Gamma\left(U, L^{*}\right)$ is a prespectrum of simplicial sets. The following result is an immediate consequence of Lemma 13.8.

COROLlary 13.9. - With the above conditions and notations, assume in addition that for any $x \in X$ the geometric realization of the prespectrum of stalks $L_{x}^{*}$ at $x$ is an $\Omega$-prespectrum. Then for any open $U \subset X$, the prespectrum $R \Gamma\left(U, L^{*}\right)$ is also an $\Omega$-prespectrum.

Consistent with the notation (13.0), we denote by $\Omega^{-i} \mathcal{K}^{q}$ the simplicial sheaf associated to the presheaf sending $U$ to $\underbrace{S_{\bullet} \ldots S_{\bullet}}_{i}{ }^{\mathcal{Q}, \mathbb{A}^{q}}\left(\Delta^{\bullet} \times U\right) \equiv w \underbrace{S_{\bullet} \ldots S_{\bullet}}_{i}\left(\mathcal{C P}^{\mathcal{Q}}\left(\Delta^{\bullet} \times U \times \mathbb{A}^{q}\right)\right)$. 
As seen in (C.1), the natural maps $\Sigma \Omega^{-i} \mathcal{K}^{q} \rightarrow \Omega^{-i-1} \mathcal{K}^{q}$ have the property that for any point $x \in X$ the induced map of topological spaces

$$
\left|\Omega^{-i} \mathcal{K}_{x}^{q}\right| \rightarrow \Omega\left(\left|\Omega^{-i-1} \mathcal{K}_{x}^{q}\right|\right)
$$

is a weak equivalence. Corollary 13.9 implies now the following result.

Proposition 13.10. - For any open subset $U \subset X$ and any $q \geqslant 0$ the sequence $\left|R \Gamma\left(U, \Omega^{-i} \mathcal{K}^{q}\right)\right|$ is an $\Omega$-prespectrum. In particular $\left|R \Gamma\left(U, \Omega^{-i} \mathcal{K}^{q}\right)\right|$ is an infinite loop space for every $q \geqslant 0, i \geqslant 1$. Moreover the space $\left|R \Gamma\left(U, \Omega^{-i-1} \mathcal{K}^{q}\right)\right|$ is connected for any $U$ and $i \geqslant 1$ and hence is a connected delooping of the space $\left|R \Gamma\left(U, \Omega^{-i} \mathcal{K}^{q}\right)\right|$. The same conclusions also apply to the prespectrum $\left|R \Gamma(U, \underbrace{B \ldots B_{\bullet}}_{i}\left(C_{\bullet}\left(\mathbb{Z}^{\mathcal{Q}, \mathbb{A}^{q}}\right)\right))\right|$.

Proof.-Only the connectedness of the above spaces remains to be proved. Corollary 13.3.1 and Lemma B.3 give the following answer for the homotopy groups of $\left|R \Gamma(U, \underbrace{B \bullet \ldots B_{\bullet}}_{i}\left(C_{\bullet}\left(\mathbb{Z}^{\mathcal{Q}, \mathbb{A}^{q}}\right)\right))\right|:$

$$
\pi_{p}\left(\left|R \Gamma\left(U, B \bullet \ldots B \bullet\left(C \bullet\left(\mathbb{Z}^{\mathcal{Q}, \mathbb{A}^{q}}\right)\right)\right)\right|=H^{-p+i}\left(U, C^{*}\left(\mathbb{Z}^{\mathcal{Q}, \mathbb{A}^{q}}\right)\right)\right)=C H^{q}(U, p-i)
$$

This computation implies that the space $\left|R \Gamma\left(U, B \bullet \ldots B_{\bullet}\left(C_{\bullet}\left(\mathbb{Z}^{\mathcal{Q}, \mathbb{A}^{q}}\right)\right)\right)\right|$ is connected and for $i>1$ even simply connected. We verify the connectedness of the space $\left|R \Gamma\left(U, \Omega^{-i} \mathcal{K}^{q}\right)\right|$ by induction on $q$. For $q=0$, this follows from the explicit description of $\pi_{0}$ arising from Waldhausen's construction showing that $\mid w S_{\bullet} \ldots S_{\bullet}\left(\mathcal{C P}\left(U \times \Delta^{\bullet}\right) \mid\right.$ is connected, together with Mayer-Vietoris for $K$-theory, which implies (see [9] Theorem 4) that the canonical map

$$
w S_{\bullet} \ldots S_{\bullet}\left(\mathcal{C P}\left(U \times \Delta^{\bullet}\right)\right) \rightarrow R \Gamma\left(U, \Omega^{-i} \mathcal{K}^{q}\right)
$$

is a weak equivalence. To make the inductive step (for $i>1$ ) we note that the same argument as at the beginning of this section shows that for any $i \geqslant 1$ the sequence

$$
R \Gamma\left(\Omega^{-i} \mathcal{K}^{q+1}\right) \rightarrow R \Gamma\left(\Omega^{-i} \mathcal{K}^{q}\right) \rightarrow R \Gamma\left(B \bullet \ldots B \bullet\left(C \bullet\left(\mathbb{Z}^{\mathcal{Q}, \mathbb{A}^{q}}\right)\right)\right)
$$

is a homotopy fibration sequence and consider the corresponding long exact sequence in homotopy.

Denote by $\mathcal{K}^{q}$ the prespectrum of simplicial sheaves on $X$ with terms $\Omega^{-i} \mathcal{K}^{q}$. Proposition 13.10 shows that $\left|R \Gamma\left(U, \mathcal{K}^{q}\right)\right|$ is a $(-1)$-connected $\Omega$-prespectrum (for any open $U \subset X$ ). Moreover for every $q \geqslant 0$ we have a homotopy fibration sequence of prespectra

$$
R \Gamma\left(X, \mathcal{K}^{q+1}\right) \rightarrow R \Gamma\left(X, \mathcal{K}^{q}\right) \rightarrow R \Gamma\left(X, \mathbb{B}\left(C \bullet\left(\mathbb{Z}^{\mathcal{Q}, \mathbb{A}^{q}}\right)\right)\right)
$$

Corollary 6.1.1 implies now the following proposition.

PROPOSITION 13.11. - For any $i>0$, the tower of spaces

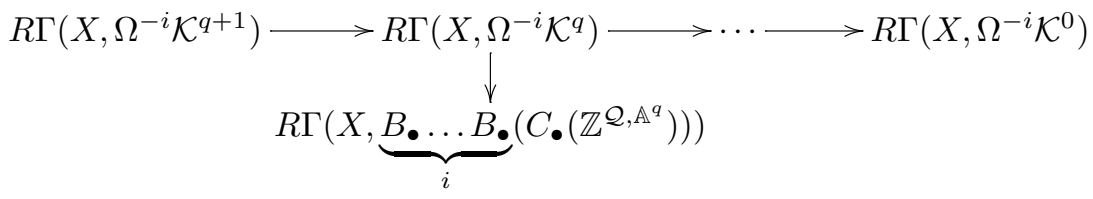


determines an exact couple and a strongly convergent spectral sequence, which coincides (after appropriate re-indexing) with the spectral sequence

$$
E_{2}^{p, q}=H^{p-q}(X, \mathbb{Z}(-q))=C H^{-q}(X,-p-q) \quad \Rightarrow \quad K_{-p-q}(X)
$$

of Theorem 13.6.

We finish this section with the following version of the spectral sequence (13.6.1) for nonsmooth schemes.

THEOREM 13.12. - Let $Z$ be a (not necessarily smooth) equidimensional quasiprojective scheme. Then there exists a strongly convergent spectral sequence

$$
E_{2}^{p, q}=C H^{-q}(Z,-p-q) \quad \Longrightarrow \quad K_{-p-q}^{\prime}(Z)
$$

where $K_{*}^{\prime}(X)$ denotes the Quillen $K$-theory of the exact category of coherent sheaves on $Z$.

Proof.-Embed $Z$ as a closed subscheme in a smooth irreducible scheme $X$ and set $U=X \backslash Z$. For any simplicial sheaf $A_{\bullet}$ on $X$ let $R \Gamma_{Z}\left(X, A_{\bullet}\right)$ be the fiber over the distinguished point of the Kan fibration

$$
R \Gamma\left(X, A_{\bullet}\right)=\Gamma\left(X, \mathcal{R}\left(A_{\bullet}\right)\right) \rightarrow R \Gamma\left(U, A_{\bullet}\right)=\Gamma\left(U, \mathcal{R}\left(A_{\bullet}\right)\right)
$$

Consider the following commutative diagram

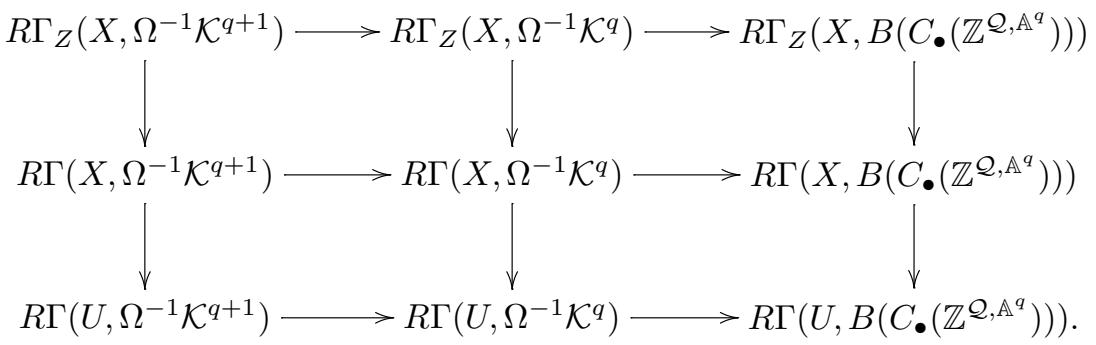

All columns of this diagram and all rows except possibly for the top one are homotopy fibration sequences which implies that the top row is a homotopy fibration sequence as well. Thus we get a tower of homotopy fibration sequences

$$
\begin{aligned}
R \Gamma_{Z}\left(X, \Omega^{-1} \mathcal{K}^{q+1}\right) \longrightarrow & R \Gamma_{Z}\left(X, \Omega^{-1} \mathcal{K}^{q}\right) \longrightarrow \cdots \longrightarrow \Gamma_{Z}\left(X, \Omega^{-1} \mathcal{K}^{0}\right) . \\
\downarrow & R \Gamma_{Z}\left(X, B\left(C \bullet\left(\mathbb{Z}^{\mathcal{Q}, \mathbb{A}^{q}}\right)\right)\right)
\end{aligned}
$$

Moreover the previous results imply immediately that the spaces $R \Gamma_{Z}\left(X, \Omega^{-1} \mathcal{K}^{q}\right)$ (and also $\left.R \Gamma_{Z}\left(X, B\left(C_{\bullet}\left(\mathbb{Z}^{\mathcal{Q}, \mathbb{A}^{q}}\right)\right)\right)\right)$ are connected infinite loop spaces and are at least $(q-\operatorname{dim} X-1)$ connected. Thus Proposition 6.1 yields a strongly convergent spectral sequence. Quillen's Localization Theorem implies immediately that the limit of this spectral sequence coincides with $K_{*}^{\prime}(Z)$ and Bloch's Localization Theorem [4] implies that the $E_{2}$-term consists of higher Chow groups of $Z$.

$4^{\mathrm{e}}$ SÉRIE - TOME $35-2002-\mathrm{N}^{\circ} 6$ 


\section{Multiplicative structure for the homotopy spectral sequence}

The purpose of this section is to formulate an existence theorem, Theorem 14.4, for the pairing of homotopy spectral sequences as in Proposition 6.1. The basis for such an existence proof is a classical construction of Massey, recalled in Theorem 14.1. Since the multiplicative structure of the motivic spectral sequence with finite coefficients is of particular interest, we are careful to formulate Theorem 14.4 so that it is applicable to the finite coefficients analogue of the spectral sequence of Proposition 6.1.

The following general result concerning the multiplicative properties of the spectral sequence defined by an exact couple is an extraction from a paper of Massey [18].

TheOREM 14.1. - Let $\left(D^{2}, E^{2} ; i, j, k\right),\left(D^{\prime 2}, E^{\prime 2} ; i^{\prime}, j^{\prime}, k^{\prime}\right),\left(D^{\prime \prime 2}, E^{\prime \prime 2} ; i^{\prime \prime}, j^{\prime \prime}, k^{\prime \prime}\right)$ be three exact couples (with degrees of $i, j, k$ being equal to $(1,-1),(0,0),(-2,1)$ respectively). Assume that we are given bilinear pairings (respecting bidegrees) $E^{\prime 2} \otimes E^{\prime \prime 2} \rightarrow E^{2}$ and the following condition is satisfied for all $n \geqslant 0$ :

Condition $\mu_{n}$ : For any bihomogenous elements $b^{\prime} \in E^{\prime 2}, b^{\prime \prime} \in E^{\prime \prime 2}$ and any bihomogenous elements $x^{\prime} \in D^{\prime 2}, x^{\prime \prime} \in D^{\prime \prime 2}$ such that $k^{\prime}\left(b^{\prime}\right)=\left(i^{\prime}\right)^{n}\left(x^{\prime}\right), k^{\prime \prime}\left(b^{\prime \prime}\right)=\left(i^{\prime \prime}\right)^{n}\left(x^{\prime \prime}\right)$, there exists $x \in D^{2}$ for which $k\left(b^{\prime} \cdot b^{\prime \prime}\right)=i^{n}(x), j(x)=j^{\prime}\left(x^{\prime}\right) \cdot b^{\prime \prime}+(-1)^{\text {degb }} b^{\prime} \cdot j^{\prime \prime}\left(x^{\prime \prime}\right)$.

Then for all $r \geqslant 2$ we have natural pairings $E^{\prime r} \otimes E^{\prime \prime r} \stackrel{\left(y^{\prime}, y^{\prime \prime}\right) \mapsto y^{\prime} \cdot y^{\prime \prime}}{\longrightarrow} E^{r}$, the differentials $d_{r}$ are "derivations" in the sense that $d_{r}\left(y^{\prime} \cdot y^{\prime \prime}\right)=d_{r}^{\prime}\left(y^{\prime}\right) \cdot y^{\prime \prime}+(-1)^{\operatorname{deg} y^{\prime}} y^{\prime} \cdot d_{r}^{\prime \prime}\left(y^{\prime \prime}\right)$, and the isomorphisms $E^{\prime r+1}=H\left(E^{\prime r}, d^{\prime r}\right), E^{\prime \prime r+1}=H\left(E^{\prime \prime r}, d^{\prime \prime r}\right), E^{r+1}=H\left(E^{r}, d^{r}\right)$ are compatible with pairings.

Assume further that all three exact couples are bounded (i.e., for any $n$ there exist $f(n)$ and $g(n)$ such that $D_{p, n-p}^{2}=0$ for $p<f(n)$ and $D_{p, n-p}^{2} \stackrel{i}{\rightarrow} D_{p+1, n-p-1}^{2}$ is an isomorphism for $p>g(n))$. Then the spectral sequences converge strongly to $H^{\prime}, H^{\prime \prime}$ and $H$ respectively, where $H_{n}=\lim _{p} D_{p, n-p}^{2}$ provided with the filtration $F_{p} H_{n}=\operatorname{Im}\left(D_{p, n-p}^{2} \rightarrow H_{n}\right)$.

Assume finally that we are also given pairings $D^{\prime 2} \otimes D^{\prime \prime 2} \stackrel{p, n-p}{\rightarrow} D^{2}$, which are compatible with the $j$-maps, i.e., the following diagram commutes

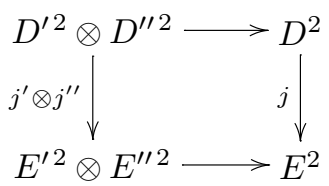

and the map $i$ is a transducer, i.e.,

$$
i\left(a^{\prime} \cdot a^{\prime \prime}\right)=i^{\prime}\left(a^{\prime}\right) \cdot a^{\prime \prime}=a^{\prime} \cdot i^{\prime \prime}\left(a^{\prime \prime}\right) .
$$

In this case we get a canonical pairing ${H^{\prime}}{ }_{*} \otimes H^{\prime \prime}{ }_{*} \rightarrow H_{*}$ compatible with filtrations, i.e., $F_{p^{\prime}}\left(H^{\prime}{ }_{*}\right) \cdot F_{p^{\prime \prime}}\left(H^{\prime \prime}{ }_{*}\right) \subset F_{p^{\prime}+p^{\prime \prime}}\left(H_{*}\right)$ and hence also the induced pairing on the associated graded groups. Moreover the isomorphisms $F_{p / p-1} H_{p+q} \stackrel{\sim}{\rightarrow} E_{p q}^{\infty}$ are compatible with pairings.

Whenever the conditions of Theorem 14.1 are satisfied we shall say that we have a pairing of exact couples. Thus, Theorem 14.1 may be restated in short by saying that a pairing of exact couples defines a pairing of associated spectral sequences.

We shall apply Massey's theorem above not only to spectral sequences which arise as in Proposition 6.1 but also their analogue involving homotopy groups with finite coefficients. More generally consider a finite pointed $C W$-complex $M$ of dimension $d$. For any pointed space $X$ 
set

$$
\pi_{i}(X, M)=\left[S^{i-d} \wedge M, X\right] \text { if } i \geqslant d .
$$

Thus $\pi_{i}(X, M)$ is defined only for $i \geqslant d, \pi_{d}(X, M)$ is only a pointed set, $\pi_{i}(X, M)$ is a group for $i>d$, which is abelian provided that $i>d+1$ or $X$ is an $H$-space. For any homotopy fibration $X^{\prime} \rightarrow X \rightarrow B$ we get a long exact sequence of homotopy groups with coefficients in $M$

$$
\begin{aligned}
\pi_{i}\left(X^{\prime}, M\right) & \rightarrow \pi_{i}(X, M) \rightarrow \pi_{i}(B, M) \stackrel{\partial}{\rightarrow} \pi_{i-1}\left(X^{\prime}, M\right) \rightarrow \cdots \\
& \rightarrow \pi_{d}\left(X^{\prime}, M\right) \rightarrow \pi_{d}(X, M) \rightarrow \pi_{d}(B, M) .
\end{aligned}
$$

All terms of this sequence except the last three are groups and all the maps not involving these three last terms are group homomorphisms. Note also that $\pi_{i}(X, M)=0$ for all $i \leqslant n$ provided that $X$ is $n$-connected. With these remarks the proof of the following statement becomes identical to that of Proposition 6.1.

Proposition 14.2. - With the conditions and notations of Proposition 6.1, assume further that $M$ is a pointed finite $C W$-complex such that

(1) $\left[M, X_{q}\right]=\left[M, B_{q}\right]=*$ for all $q \geqslant 0$.

(2) The groups $\left[\Sigma M, X_{q}\right]$ and $\left[\Sigma M, B_{q}\right]$ are abelian for all $q \geqslant 0$.

In this case, there exists a strongly convergent spectral sequence

$$
E_{p q}^{2}=\pi_{p+q}\left(B_{q}, M\right) \quad \Rightarrow \quad \pi_{p+q}(X, M) .
$$

The following standard homotopy result is useful for the computation of the map

$$
\partial:[\Sigma M, B] \rightarrow[M, Y]
$$

in the case of a homotopy fibration. In what follows we identify the (reduced) suspension $\Sigma M$ with $S^{1} \wedge M$, where $S^{1}=\mathrm{I} /\{0,1\}$. We consider I as a pointed space with a distinguished point $0 \in \mathrm{I}$ and we denote by $C M=\mathrm{I} \wedge M$ the reduced cone over $M$, so that $\Sigma M=C M / M$.

LEMMA 14.3.0. - Let $Y \stackrel{f}{\rightarrow} X \stackrel{p}{\rightarrow} B$ be a sequence of continuous maps of pointed spaces. Assume that the composition map pf is trivial and the induced map from $Y$ to the homotopy fiber of $p$ is a weak equivalence. Let further $M$ be a finite pointed $C W$-complex of dimension $d$.

(1) Let $w: M \rightarrow Y, v: C M \rightarrow X$ be continuous maps of pointed spaces such that, $v_{\mid M}=f \circ w$. Then $p \circ v$ contracts $M$ to the distinguished point $*$ of $B$, thus defining a map (which we still write as $p \circ v) \Sigma M \stackrel{p \circ v}{\longrightarrow} B$ and $\partial([p \circ v])=[w] \in[M, Y]$.

(2) Let $b \in[\Sigma M, B], y \in[M, Y]$ be elements such that $\partial(b)=y$ and let $w: M \rightarrow Y$ be $a$ representative of $y$. Then there exists a pointed map $v: C M \rightarrow X$ for which $v_{\mid M}=f \circ w$, $b=[p \circ v]$.

In conditions and notations of Lemma 14.3.0 assume that we are given a map

$$
u: \Sigma^{k+l} M=S^{k} \wedge S^{l} \wedge M \rightarrow B
$$

and a lifting

$$
v:(\mathrm{I} \wedge \mathrm{I}) \wedge \Sigma^{k+l-2} M=\left(\mathrm{I} \wedge S^{k-1}\right) \wedge\left(\mathrm{I} \wedge S^{l-1}\right) \wedge M \rightarrow X
$$

for $u$. Assume finally that we are given a map $w: \partial(\mathrm{I} \wedge \mathrm{I}) \wedge \Sigma^{k+l-2} M \rightarrow Y$ such that

$$
v_{\mid \partial(I \wedge I) \wedge \Sigma^{k+l-2} M}=f \circ w .
$$


In what follows we identify $\partial(\mathrm{I} \wedge \mathrm{I})$ with $S^{1}$. To do so we just need to fix the orientation of $\partial(\mathrm{I} \wedge \mathrm{I})$, which we do by requiring that we first go through $1 \times \mathrm{I}$ in the standard direction and then go through $\mathrm{I} \times 1$ in the reverse. Now we may identify $\partial(\mathrm{I} \wedge \mathrm{I}) \wedge \Sigma^{k+l-2} M$ with $\Sigma^{k+l-1} M$ which we do positioning $\partial(\mathrm{I} \wedge \mathrm{I})$ as the $k$ th suspension coordinate. We leave the proof of the following elementary Lemma to the reader.

LEMMA 14.3.1. - In the above conditions and notations we have the following formula

$$
\partial([u])=[w] \in \pi_{k+l-1+d}(Y, M) .
$$

Assume we are given three finite pointed $C W$-complexes $M^{\prime}, M^{\prime \prime}$ and $M$ of dimensions $d^{\prime}, d^{\prime \prime}$ and $d$ respectively (with $d^{\prime}+d^{\prime \prime} \geqslant d$ ) and a pointed map $\Sigma^{d^{\prime}+d^{\prime \prime}-d} M \rightarrow M^{\prime} \wedge M^{\prime \prime}$. Assume further that we have also three pointed spaces $X^{\prime}, X^{\prime \prime}, X$ and a pairing of spaces $X^{\prime} \wedge X^{\prime \prime} \rightarrow X$. In this case every pair of continuous pointed maps $\Sigma^{i-d^{\prime}} M^{\prime} \stackrel{f^{\prime}}{\rightarrow} X^{\prime}, \Sigma^{j-d^{\prime \prime}} M^{\prime \prime} \stackrel{f^{\prime \prime}}{\rightarrow} X^{\prime \prime}\left(i \geqslant d^{\prime}\right.$, $j \geqslant d^{\prime \prime}$ ) defines a pointed continuous map (which we denote by $f^{\prime} \cdot f^{\prime \prime}$ )

$$
\begin{aligned}
f^{\prime} \cdot f^{\prime \prime}: \Sigma^{i+j-d} M & =\Sigma^{i+j-d^{\prime}-d^{\prime \prime}} \Sigma^{d^{\prime}+d^{\prime \prime}-d} M \rightarrow \Sigma^{i+j-d^{\prime}-d^{\prime \prime}}\left(M^{\prime} \wedge M^{\prime \prime}\right) \\
& =\Sigma^{i-d^{\prime}} M^{\prime} \wedge \Sigma^{j-d^{\prime \prime}} M^{\prime \prime} \stackrel{f^{\prime} \wedge f^{\prime \prime}}{\longrightarrow} X^{\prime} \wedge X^{\prime \prime} \rightarrow X
\end{aligned}
$$

A straightforward verification shows that the above pairing on maps respects homotopy and thus we get pairings $\pi_{i}\left(X^{\prime}, M^{\prime}\right) \times \pi_{j}\left(X^{\prime \prime}, M^{\prime \prime}\right) \rightarrow \pi_{i+j}(X, M)$. Moreover the above pairings are bilinear provided that $i>d^{\prime}, j>d^{\prime \prime}$.

As seen in the next proposition, this leads to pairings of spectral sequences converging to homotopy groups with coefficients.

Proposition 14.3. - Assume that we are given three finite pointed $C W$-complexes $M^{\prime}, M^{\prime \prime}$ and $M$ of dimensions $d^{\prime}, d^{\prime \prime}$ and $d$ respectively (with $d^{\prime}+d^{\prime \prime} \geqslant d$ ) and a pointed map $\Sigma^{d^{\prime}+d^{\prime \prime}-d} M \rightarrow M^{\prime} \wedge M^{\prime \prime}$. Assume further that we are given three towers $\left(X^{\prime}, B^{\prime}\right),\left(X^{\prime \prime}, B^{\prime \prime}\right)$, $(X, B)$ as in Proposition 6.1 satisfying the conditions of Proposition 14.2 with respect to $M^{\prime}$, $M^{\prime \prime}$ and $M$ respectively. Assume finally that for all $q^{\prime}, q^{\prime \prime} \geqslant 0$, we are given pairings of spaces

$$
X_{q^{\prime}}^{\prime} \wedge X_{q^{\prime \prime}}^{\prime \prime} \rightarrow X_{q^{\prime}+q^{\prime \prime}}, \quad B_{q^{\prime}}^{\prime} \wedge B_{q^{\prime \prime}}^{\prime \prime} \rightarrow B_{q^{\prime}+q^{\prime \prime}}
$$

which fit in commutative diagrams
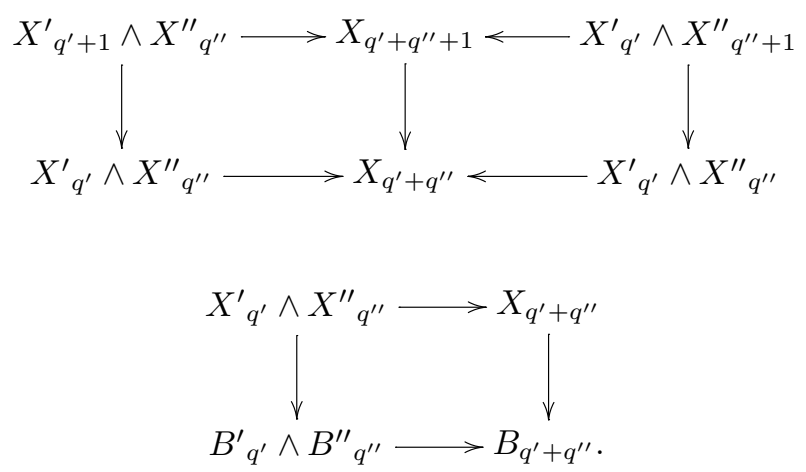

Then the above pairings of spaces induce natural pairings of the associated homotopy exact couples, and hence of the associated spectral sequences. 
Proof. - As was explained above, the given pairings of spaces induce pairings in homotopy groups, thus defining products $D^{\prime 2} \otimes D^{\prime \prime 2} \rightarrow D^{2}, E^{\prime 2} \otimes E^{\prime \prime 2} \rightarrow E^{2}$. Moreover these products are obviously compatible with the $j$-homomorphisms and the homomorphism $i$ is obviously a transducer. Thus we only need to check the validity of the condition $\mu_{r}(r \geqslant 0)$ of Theorem 14.1. Let $b^{\prime} \in \pi_{n}\left(B^{\prime}{ }_{q^{\prime}}, M^{\prime}\right), x^{\prime} \in \pi_{n-1}\left(X_{q^{\prime}+r+1}^{\prime}, M^{\prime}\right), b^{\prime \prime} \in \pi_{m}\left(B^{\prime \prime}{ }_{q^{\prime \prime}}, M^{\prime \prime}\right)$, $x^{\prime \prime} \in \pi_{m-1}\left(X^{\prime \prime} q^{\prime \prime}+r+1, M^{\prime \prime}\right)\left(n>d^{\prime}, m>d^{\prime \prime}\right)$ be elements such that

$$
\begin{aligned}
& \partial\left(b^{\prime}\right)=\left(i^{\prime}\right)^{r}\left(x^{\prime}\right)=\left(f_{q^{\prime}+2}^{\prime} \circ \cdots \circ f_{q^{\prime}+r+1}^{\prime}\right)_{*}\left(x^{\prime}\right), \\
& \partial\left(b^{\prime \prime}\right)=\left(i^{\prime \prime}\right)^{r}\left(x^{\prime \prime}\right)=\left(f_{q^{\prime \prime}+2}^{\prime \prime} \circ \cdots \circ f_{q^{\prime \prime}+r+1}^{\prime \prime}\right)_{*}\left(x^{\prime \prime}\right) .
\end{aligned}
$$

Represent $x^{\prime}$ (respectively $x^{\prime \prime}$ ) by

$$
w^{\prime}: \Sigma^{n-d^{\prime}-1} M^{\prime} \rightarrow X_{q^{\prime}+r+1}^{\prime}
$$

(respectively by $w^{\prime \prime}: \Sigma^{m-d^{\prime \prime}-1} M^{\prime \prime} \rightarrow X_{q^{\prime \prime}+r+1}^{\prime \prime}$ ). According to Lemma 14.3.0 there exist maps

$$
v^{\prime}: C \Sigma^{n-d^{\prime}-1} M^{\prime} \rightarrow X_{q^{\prime}}^{\prime}, \quad v^{\prime \prime}: C \Sigma^{m-d^{\prime \prime}-1} M^{\prime \prime} \rightarrow X_{q^{\prime \prime}}^{\prime \prime}
$$

such that

$$
\begin{aligned}
& v_{\mid \Sigma^{n-d^{\prime}-1} M^{\prime}}^{\prime}=f_{q^{\prime}+1}^{\prime} \circ \cdots \circ f_{q^{\prime}+r+1}^{\prime} \circ w^{\prime}, \quad b^{\prime}=\left[p_{q^{\prime}}^{\prime} \circ v^{\prime}\right], \\
& v_{\mid \Sigma^{m-d^{\prime \prime}-1} M^{\prime \prime}}^{\prime \prime}=f_{q^{\prime \prime}+1}^{\prime \prime} \circ \cdots \circ f_{q^{\prime \prime}+r+1}^{\prime \prime} \circ w^{\prime \prime}, \quad b^{\prime \prime}=\left[p_{q^{\prime \prime}}^{\prime \prime} \circ v^{\prime \prime}\right] .
\end{aligned}
$$

Denote the map

$$
p_{q^{\prime}}^{\prime} \circ v^{\prime}: \Sigma^{n-d^{\prime}} M^{\prime}=C \Sigma^{n-d^{\prime}-1} M^{\prime} / \Sigma^{n-d^{\prime}-1} M^{\prime} \rightarrow B_{q^{\prime}}^{\prime}
$$

(respectively $\left.p_{q^{\prime \prime}}^{\prime \prime} \circ v^{\prime \prime}: \Sigma^{m-d^{\prime \prime}} M^{\prime \prime}=C \Sigma^{m-d^{\prime \prime}-1} M^{\prime \prime} / \Sigma^{m-d^{\prime \prime}-1} M^{\prime \prime} \rightarrow B_{q^{\prime \prime}}^{\prime \prime}\right)$ by $u^{\prime}$ (respectively by $\left.u^{\prime \prime}\right)$, so that $b^{\prime}=\left[u^{\prime}\right], b^{\prime \prime}=\left[u^{\prime \prime}\right]$. The map $u=u^{\prime} \cdot u^{\prime \prime}: \Sigma^{n+m-d} M \rightarrow B_{q^{\prime}+q^{\prime \prime}}$ is a representative for $b^{\prime} \cdot b^{\prime \prime}$ and the map $v=v^{\prime} \cdot v^{\prime \prime}:(\mathrm{I} \wedge \mathrm{I}) \wedge \Sigma^{n+m-d-2} M \rightarrow X_{q^{\prime}+q^{\prime \prime}}$ is a lifting of $u$. Consider the restriction of $v$ to $\partial(\mathrm{I} \wedge \mathrm{I}) \wedge \Sigma^{n+m-d-2} M$. Observe that $\partial(\mathrm{I} \wedge \mathrm{I})=1 \times \mathrm{I} \cup \mathrm{I} \times 1$. The restrictions of $v$ to $(1 \times \mathrm{I}) \wedge \Sigma^{n+m-d-2} M$ and to $(\mathrm{I} \times 1) \wedge \Sigma^{n+m-d-2} M$ are given by the formulas:

$$
\begin{aligned}
& v_{\mid(1 \times I) \wedge \Sigma^{n+m-d-2} M}=\left(\left(f^{\prime}\right)^{r+1} \circ w^{\prime}\right) \cdot v^{\prime \prime}=f^{r+1} \circ\left(w^{\prime} \cdot v^{\prime \prime}\right), \\
& v_{\mid(I \times 1) \wedge \Sigma^{n+m-d-2} M}=v^{\prime} \cdot\left(\left(f^{\prime \prime}\right)^{r+1} \circ w^{\prime \prime}\right)=f^{r+1} \circ\left(v^{\prime} \cdot w^{\prime \prime}\right) .
\end{aligned}
$$

Define a map

$$
w: \Sigma^{n+m-d-1} M=(\partial(\mathrm{I} \wedge \mathrm{I})) \wedge \Sigma^{n+m-d-2} M \rightarrow X_{q^{\prime}+q^{\prime \prime}+r+1}
$$

requiring that

$$
w_{\mid(1 \times I) \wedge \Sigma^{n+m-d-2} M}=w^{\prime} \cdot v^{\prime \prime}, \quad w_{\mid(I \times 1) \wedge \Sigma^{n+m-d-2} M}=v^{\prime} \cdot w^{\prime \prime} .
$$

Note that the restrictions of $w^{\prime} \cdot v^{\prime \prime}$ and of $v^{\prime} \cdot w^{\prime \prime}$ to $(1 \times 1) \times \Sigma^{n+m-d-2} M$ are both equal to $f^{r+1} \circ\left(w^{\prime} \cdot w^{\prime \prime}\right)$. Thus the above partial data for $w$ are compatible one with another and hence the map $w$ is well defined. Furthermore

$$
f_{q^{\prime}+q^{\prime \prime}+1} \circ \cdots \circ f_{q^{\prime}+q^{\prime \prime}+r+1} \circ w=\left(v^{\prime} \cdot v^{\prime \prime}\right)_{\mid \partial(I \wedge I) \wedge \Sigma^{n+m-d-2} M} .
$$


Lemma 14.3.1 implies that $\left[f_{q^{\prime}+q^{\prime \prime}+2} \circ \cdots \circ f_{q^{\prime}+q^{\prime \prime}+r+1} \circ w\right]=\partial\left(b^{\prime} \cdot b^{\prime \prime}\right)$, i.e. $\partial\left(b^{\prime} \cdot b^{\prime \prime}\right)=i^{r}([w])$. Moreover $j([w])=\left[p_{q^{\prime}+q^{\prime \prime}+r+1} \circ w\right]$. The restriction of the map

$$
p \circ w:(\partial(I \wedge \mathrm{I})) \wedge \Sigma^{n+m-d-2} M \rightarrow B_{q^{\prime}+q^{\prime \prime}+r+1}
$$

to $(1 \times \mathrm{I}) \wedge \Sigma^{n+m-d-2} M$ (respectively to $\left.(\mathrm{I} \times 1) \wedge \Sigma^{n+m-d-2} M\right)$ coincides with $\left(p^{\prime} \circ w^{\prime}\right) \cdot u^{\prime \prime}$ (respectively with $u^{\prime} \cdot\left(p^{\prime \prime} \circ w^{\prime \prime}\right)$ ). In particular this map contracts $1 \times 1 \times \Sigma^{n+m-d-2} M$ to the distinguished point $*$ and hence may be factored in the form

$$
\begin{aligned}
& \partial(\mathrm{I} \wedge \mathrm{I}) \wedge \Sigma^{n+m-d-2} M \rightarrow(\partial(\mathrm{I} \wedge \mathrm{I}) /\{*, 1 \times 1\}) \wedge \Sigma^{n+m-d-2} M \\
& \quad=\left(S^{1} \vee S^{1}\right) \wedge \Sigma^{n+m-d-2} M=S^{n+m-d-1} M \vee S^{n+m-d-1} M \rightarrow B_{q^{\prime}+q^{\prime \prime}+r+1} .
\end{aligned}
$$

Thus the class of this map in $\pi_{n+m-1}\left(B_{q^{\prime}+q^{\prime \prime}+r+1}, M\right)$ is a sum of two classes, one being represented by a map $\left(p^{\prime} \circ w^{\prime}\right) \cdot u^{\prime \prime}$ and the second being represented by a map which we previously wrote (not quite accurately) as $u^{\prime} \cdot\left(p^{\prime \prime} \circ w^{\prime \prime}\right)$, but which actually differs from this product map by a cyclic permutation of the first $n-d^{\prime}+1$ suspension coordinates. Thus

$$
[p w]=\left[p^{\prime} w^{\prime}\right] \cdot\left[u^{\prime \prime}\right]+(-1)^{n-d^{\prime}}\left[u^{\prime}\right] \cdot\left[p^{\prime \prime} \circ w^{\prime \prime}\right]=j^{\prime}\left(x^{\prime}\right) \cdot b^{\prime \prime}+(-1)^{n-d^{\prime}} b^{\prime} \cdot j^{\prime \prime}\left(x^{\prime \prime}\right) .
$$

The pairings appearing in $K$-theory do not quite satisfy the conditions of Proposition 14.3. However, the $K$-theory tower of fibrations $(X, B)$ (and similarly for $X^{\prime}, X^{\prime \prime}$ ) has a much richer structure described in the following proposition.

THEOREM 14.4. - Assume that we are given three finite pointed $C W$-complexes $M^{\prime}, M^{\prime \prime}$ and $M$ of dimensions $d^{\prime}, d^{\prime \prime}$ and $d$ respectively (with $d^{\prime}+d^{\prime \prime} \geqslant d$ ) and a pointed map

$$
\Sigma^{d^{\prime}+d^{\prime \prime}-d} M \rightarrow M^{\prime} \wedge M^{\prime \prime}
$$

Assume further that we are given three towers $\left(X^{\prime}, B^{\prime}\right),\left(X^{\prime \prime}, B^{\prime \prime}\right),(X, B)$ satisfying the conditions of Proposition 14.2 with respect to $M^{\prime}, M^{\prime \prime}$ and $M$ respectively. Assume also that the tower $(X, B)$ has the following additional structure.

(1) For each $q$ we have $q+1$ maps

$$
f_{q+1}^{0}, f_{q+1}^{1}, \ldots, f_{q+1}^{q}: X_{q+1} \rightarrow X_{q}
$$

which satisfy the simplicial relation

$$
f_{q}^{i} \circ f_{q+1}^{j}=f_{q}^{j-1} \circ f_{q+1}^{i}: X_{q+1} \rightarrow X_{q-1} \quad(0 \leqslant i<j \leqslant q) .
$$

The map $f_{q+1}: X_{q+1} \rightarrow X_{q}$ of the tower coincides with $f_{q+1}^{q}$.

(2) For each $q$ the group $\Sigma_{q}$ acts on $X_{q}$ and $B_{q}$ on the right. More precisely, for each $\sigma \in \Sigma_{q}$ we have automorphisms of $X_{q}$ and $B_{q}$ (which we both denote by the same letter $\sigma^{*}$ ) such that $(\sigma \tau)^{*}=\tau^{*} \sigma^{*}$. The projection $p_{q}: X_{q} \rightarrow B_{q}$ is $\Sigma_{q}$-equivariant.

(3) The map $f_{q+1}=f_{q+1}^{q}: X_{q+1} \rightarrow X_{q}$ is $\Sigma_{q}$-equivariant.

(4) For each $0 \leqslant i \leqslant q$ we have the following relation

$$
f_{q+1}^{i}=f_{q+1} \circ \tau_{i}^{*}: X_{q+1} \rightarrow X_{q},
$$

where $\tau_{i}=\tau_{i}^{q+1}$ is the cyclic permutation $\tau_{i}=(i+1, i+2, \ldots, q+1)$. 
(5) For each $q$ we are given a homotopy $H_{t}$ between $f_{q+1}^{q-1}, f_{q+1}^{q}: X_{q+1} \rightarrow X_{q}$ with the following properties

(i) The homotopy $f_{q} \circ H_{t}$ between $f_{q} f_{q+1}^{q-1}=f_{q}^{q-1} f_{q+1}^{q-1}$ and $f_{q} f_{q+1}^{q}=f_{q}^{q-1} f_{q+1}^{q}=$ $f_{q}^{q-1} f_{q+1}^{q-1}$ is constant.

(ii) The homotopy $p H_{t}$ between $p_{q} f_{q+1}^{q-1}=*$ and $p_{q} f_{q+1}^{q}=*$ is constant.

(6) The induced action of $\Sigma_{q}$ in the homotopy groups of $B_{q}$ with coefficients in $M$ is trivial. Assume finally that for each $q^{\prime}, q^{\prime \prime}$, we are given pairings of spaces

$$
X_{q^{\prime}}^{\prime} \wedge X_{q^{\prime \prime}}^{\prime \prime} \rightarrow X_{q^{\prime}+q^{\prime \prime}}, \quad B_{q^{\prime}}^{\prime} \wedge B_{q^{\prime \prime}}^{\prime \prime} \rightarrow B_{q^{\prime}+q^{\prime \prime}}
$$

which fit in commutative diagrams
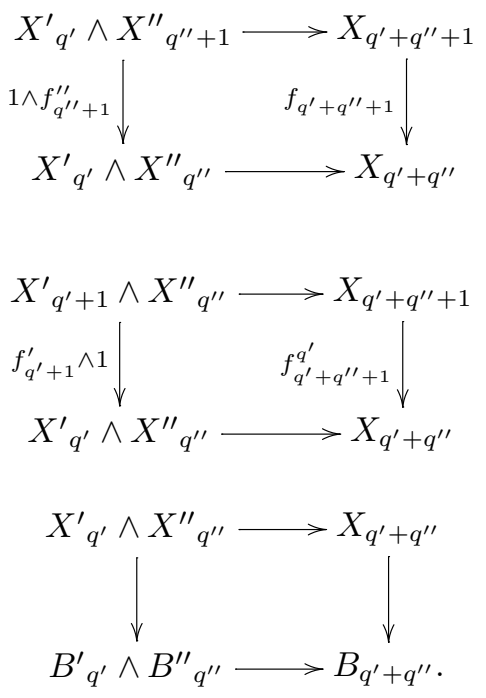

Then the above pairings of spaces induce natural pairings of the associated homotopy exact couples and hence of the associated spectral sequences as well.

Proof. - The given pairings of spaces induce (as was explained above) pairings in homotopy groups thus defining products $D^{\prime 2} \otimes D^{\prime \prime 2} \rightarrow D^{2}, E^{\prime 2} \otimes E^{\prime \prime 2} \rightarrow E^{2}$. Moreover these products are obviously compatible with the $j$-homomorphisms. To see that $i$ is a transducer it suffices (in view of the commutativity of (14.4.0) and (14.4.1)) to show that all the maps $f_{q+1}^{i}$ are homotopic one to another.

Sublemma 14.5. - For any $0 \leqslant i<j \leqslant q$ the maps $f_{q+1}^{i}, f_{q+1}^{j}: X_{q+1} \rightarrow X_{q}$ are homotopic. Moreover the corresponding homotopy becomes constant being composed with $p: X_{q} \rightarrow B_{q}$ and with $f^{\circ(q-i)}: X_{q} \rightarrow X_{i}$.

Proof. - It suffices to consider the case $j=i+1$. For $i=q-1$ the required homotopy is already given. In the general case we show that there exist $\tau \in \Sigma_{q}$ (not moving $1, \ldots, i$ ) and $\sigma \in \Sigma_{q+1}$ such that $f^{i}=\tau^{*} f^{q-1} \sigma^{*}, f^{i+1}=\tau^{*} f^{q} \sigma^{*}$. The above relations amount to the following relations in the symmetric group

$$
\tau_{i}=\sigma \tau_{q-1} \tau, \quad \tau_{i+1}=\sigma \tau .
$$

Thus $\sigma=\tau_{i+1} \tau^{-1}$ and we are left with one equation $\tau_{i+1}^{-1} \tau_{i}=\tau^{-1}(q, q+1) \tau\left(=\left(\tau^{-1}(q)\right.\right.$, $q+1))$. An easy computation shows that $\tau_{i+1}^{-1} \tau_{i}=(i+1, q+1)$ and hence we have only one 
requirement for $\tau: \tau(i+1)=q$. Having chosen $\tau$ and $\sigma$ we get the homotopy $\tau^{*} H_{t} \sigma^{*}$ relating $f^{i}$ and $f^{i+1}$. This homotopy obviously becomes constant being composed with $p$. To see that it becomes constant being composed with $f^{\circ(q-i)}$ it suffices to show that

$$
f^{\circ(q-i)} \circ \tau^{*}=f^{\circ(q-i)}: X_{q} \rightarrow X_{i}
$$

provided that $\tau \in \Sigma_{q}$ does not move the indices $1, \ldots, i$. The last fact is proved by an easy inverse induction on $i$ starting with the observation that

$$
f^{\circ 2}=f_{q-1}^{q-2} \circ f_{q}^{q-1}=f_{q-1}^{q-2} \circ f_{q}^{q-2}=f_{q-1}^{q-2} \circ f_{q}^{q-1} \circ(q-1, q)^{*}=f^{\circ 2} \circ(q-1, q)^{*} .
$$

To complete the proof of Theorem 14.4, it thus suffices to check the condition $\mu_{r}(r \geqslant 0)$. We proceed in the same way as in the proof of the Proposition 14.3. Let $b^{\prime}, b^{\prime \prime} ; x^{\prime}, x^{\prime \prime}$; $w^{\prime}, w^{\prime \prime} ; v^{\prime}, v^{\prime \prime} ; u^{\prime}, u^{\prime \prime} ; u, v$ have the same meaning as in that proof. The restrictions of $v$ to $(\mathrm{I} \times 1) \wedge \Sigma^{n+m-d-2} M$ and to $(1 \times \mathrm{I}) \wedge \Sigma^{n+m-d-2} M$ are given by the formulas:

$$
\begin{gathered}
v_{\mid(I \times 1) \wedge \Sigma^{n+m-d-2} M}=v^{\prime} \cdot\left(\left(f^{\prime \prime}\right)^{\circ(r+1)} \circ w^{\prime \prime}\right)=f^{\circ(r+1)} \circ\left(v^{\prime} \cdot w^{\prime \prime}\right), \\
v_{\mid(1 \times I) \wedge \Sigma^{n+m-d-2} M}=\left(\left(f^{\prime}\right)^{\circ(r+1)} \circ w^{\prime}\right) \cdot v^{\prime \prime}=f_{q^{\prime}+q^{\prime \prime}+1}^{q^{\prime}} \circ \cdots \circ f_{q^{\prime}+q^{\prime \prime}+r+1}^{q^{\prime}+r} \circ\left(w^{\prime} \cdot v^{\prime \prime}\right) \\
=f^{\circ(r+1)} \circ \tau^{*} \circ\left(w^{\prime} \cdot v^{\prime \prime}\right),
\end{gathered}
$$

where $\tau=\tau_{q^{\prime}+r}^{q^{\prime}+q^{\prime \prime}+r+1} \circ \cdots \circ \tau_{q^{\prime}}^{q^{\prime}+q^{\prime \prime}+1} \in \Sigma_{q^{\prime}+q^{\prime \prime}+r+1}$. The restrictions of $v^{\prime} \cdot w^{\prime \prime}$ and $\tau^{*} \circ\left(w^{\prime} \cdot v^{\prime \prime}\right)$ to $(1 \times 1) \times \Sigma^{n+m-d-2} M=\Sigma^{n+m-d-2} M$ do not agree and hence we cannot patch them together to get a map $w:(\partial(\mathrm{I} \times \mathrm{I})) \wedge \Sigma^{n+m-d-2} M \rightarrow X_{q^{\prime}+q^{\prime \prime}+r+1}$ as was done in the proof of Proposition 14.3. Fortunately they are at least homotopic so that we can patch them together having modified one (or both) of them. In fact

$$
\begin{aligned}
v^{\prime} \cdot w_{\mid \Sigma^{n+m-d-2} M}^{\prime \prime} & =\left(\left(f^{\prime}\right)^{\circ(r+1)} \circ w^{\prime}\right) \cdot w^{\prime \prime} \\
& =f_{q^{\prime}+q^{\prime \prime}+r+2}^{q^{\prime}} \circ \cdots \circ f_{q^{\prime}+q^{\prime \prime}+2 r+2}^{q^{\prime}+r} \circ\left(w^{\prime} \cdot w^{\prime \prime}\right)=f^{\circ(r+1)} \circ \alpha^{*} \circ\left(w^{\prime} \cdot w^{\prime \prime}\right)
\end{aligned}
$$

where $\alpha=\tau_{q^{\prime}+r}^{q^{\prime}+q^{\prime \prime}+2 r+2} \circ \cdots \circ \tau_{q^{\prime}}^{q^{\prime}+q^{\prime \prime}+r+2} \in \Sigma_{q^{\prime}+q^{\prime \prime}+2 r+2}$.

$$
\tau^{*} \circ\left(w^{\prime} \cdot v^{\prime \prime}\right)_{\mid \Sigma^{n+m-d-2} M}=\tau^{*} \circ f^{\circ(r+1)} \circ\left(w^{\prime} \cdot w^{\prime \prime}\right)=f^{\circ(r+1)} \circ \tau^{*} \circ\left(w^{\prime} \cdot w^{\prime \prime}\right) .
$$

Denote the map $\alpha^{*} \circ\left(w^{\prime} \cdot w^{\prime \prime}\right)$ by $w_{0}$. Then $\tau^{*} \circ\left(w^{\prime} \cdot w^{\prime \prime}\right)=\lambda^{*} \circ w_{0}$ where $\lambda=\alpha^{-1} \tau$ is an order two shuffle permuting the blocks $\left\{q^{\prime}+q^{\prime \prime}+1, \ldots, q^{\prime}+q^{\prime \prime}+r+1\right\}$ and $\left\{q^{\prime}+q^{\prime \prime}+r+\right.$ $\left.2, \ldots, q^{\prime}+q^{\prime \prime}+2 r+2\right\}$. Note that

$$
f^{\circ(r+1)} \circ \lambda=f_{q^{\prime}+q^{\prime \prime}+r+2}^{q^{\prime}+q^{\prime \prime}+1} \circ \cdots \circ f_{q^{\prime}+q^{\prime \prime}+2 r+2}^{q^{\prime}+q^{\prime \prime}+r+1} .
$$

Sublemma 14.5 implies that there exists a homotopy $F_{t}$ between $f^{\circ(r+1)} \circ \lambda$ and $f^{\circ(r+1)}$ such that the homotopy $f^{\circ(r+1)} \circ F_{t}$ is constant. Extend the homotopy $F_{t} \circ w_{0}$ between $\tau^{*} \circ\left(w^{\prime} \cdot v^{\prime \prime}\right)_{\mid \Sigma^{n+m-d-2} M}$ and $v^{\prime} \cdot w_{\mid \Sigma^{n+m-d-2} M}^{\prime \prime}$ to a homotopy $G_{t}$ defined on $(1 \times \mathrm{I}) \wedge \Sigma^{n+m-d-2} M$ and starting with $G_{0}=\tau^{*} \circ\left(w^{\prime} \cdot v^{\prime \prime}\right)$. According to the construction the map $v^{\prime} \cdot w^{\prime \prime}$ on $(\mathrm{I} \times 1) \wedge \Sigma^{n+m-d-2} M$ and the map $G_{1}$ on $(1 \times \mathrm{I}) \wedge \Sigma^{n+m-d-2} M$ agree one with another and hence define a map

$$
w: \Sigma^{n+m-1} M=(\partial(\mathrm{I} \wedge \mathrm{I})) \wedge \Sigma^{n+m-d-2} M \rightarrow X_{q^{\prime}+q^{\prime \prime}+r+1} .
$$


Note further that the maps $f^{\circ(r+1)} \circ w$ and $v_{\mid(\partial(\mathrm{I} \wedge \mathrm{I})) \wedge \Sigma^{n+m-d-2} M}$ coincide outside $(1 \times \mathrm{I}) \wedge \Sigma^{n+m-d-2} M$ and there restrictions to $(1 \times \mathrm{I}) \wedge \Sigma^{n+m-d-2} M$ are related by a homotopy constant on $(1 \times\{0,1\}) \wedge \Sigma^{n+m-d-2} M$. Thus they are homotopic and hence

$$
\partial\left(b^{\prime} \cdot b^{\prime \prime}\right)=\left[f^{\circ r} \circ w\right]=i^{r}([w]) .
$$

Finally the same reasoning as in the proof of Proposition 14.3 shows that

$$
[p \circ w]=\left(\tau^{*}\right)_{*}\left(\left(j^{\prime}\left(x^{\prime}\right) \cdot b^{\prime \prime}\right)\right)+(-1)^{n-d^{\prime}} b^{\prime} \cdot j^{\prime \prime}\left(x^{\prime \prime}\right)=\left(j^{\prime}\left(x^{\prime}\right) \cdot b^{\prime \prime}\right)+(-1)^{n-d^{\prime}} b^{\prime} \cdot j^{\prime \prime}\left(x^{\prime \prime}\right) .
$$

\section{Multiplicative structure of the motivic spectral sequence}

In this section we show that the spectral sequence (13.6.1) has a natural multiplicative structure. This structure is induced in a natural way by products in the $K$-theory with supports, corresponding to the tensor product operation on vector bundles (cf. (C.2)). To define the product operation on the $K$-theory of the cosimplicial scheme $X \times \Delta^{\bullet}$, we unfortunately need to assume that the tensor product operation for (big) vector bundles is strictly functorial. Since it is not clear whether this can be always achieved, we begin this section by replacing (in the case of an affine scheme) the category of big vector bundles by an equivalent category of what may be called small vector bundles. This new category has an advantage of having strictly functorial tensor products.

Let $X$ be any scheme. Set $A=\Gamma\left(X, \mathcal{O}_{X}\right)$. By a small vector bundle on $X$ we mean a pair, consisting of an integer $n \geqslant 0$ and an idempotent matrix $\alpha \in M_{n}(A)$. In case $n=0$ the ring $M_{n}(A)$ is trivial (consists of zero only), so we shall drop $\alpha$ from the notation in this case. Since we want our category to have only one zero object we assume $\alpha \neq 0$ for $n>0$. Define morphisms of small vector bundles via the formula

$$
\operatorname{Hom}_{\mathcal{O}_{X}}((n, \alpha),(m, \beta))=\left\{\gamma \in M_{m, n}(A): \gamma=\gamma \alpha, \beta \gamma=\gamma\right\}
$$

Note further that to any small vector bundle $(n, \alpha)$ we may associate an actual vector bundle

$$
\mathcal{P}_{n, \alpha}=\operatorname{Ker}\left(\mathcal{O}_{X}^{n} \stackrel{1-\alpha}{\longrightarrow} \mathcal{O}_{X}^{n}\right)=\operatorname{Im}\left(\mathcal{O}_{X}^{n} \stackrel{\alpha}{\rightarrow} \mathcal{O}_{X}^{n}\right) \subset \mathcal{O}_{X}^{n}
$$

Clearly, $\operatorname{Hom}_{\mathcal{O}_{X}}((n, \alpha),(m, \beta))=\operatorname{Hom}_{\mathcal{O}_{X}}\left(\mathcal{P}_{n, \alpha}, \mathcal{P}_{m, \beta}\right)$. Thus the category of small vector bundles on $X$ is naturally equivalent to the category of those vector bundles $\mathcal{P}$ which are direct summands in some $\mathcal{O}_{X}^{n}$. In particular the category of small vector bundles is equivalent to the category of vector bundles in case $X$ is affine.

Let $f: Y \rightarrow X$ be any morphism of schemes. For a small vector bundle $(n, \alpha)$ on $X$ define

$$
f^{*}(n, \alpha)= \begin{cases}\left(n, f^{*}(\alpha)\right), & \text { if } f^{*}(\alpha) \neq 0 \\ 0, & \text { if } f^{*}(\alpha)=0\end{cases}
$$

where $f^{*}$ on the right denotes the canonical ring homomorphism $\Gamma\left(X, \mathcal{O}_{X}\right) \stackrel{f^{*}}{\longrightarrow} \Gamma\left(Y, \mathcal{O}_{Y}\right)$. Note that $(f g)^{*}(n, \alpha)=g^{*}\left(f^{*}(n, \alpha)\right)$ for any pair of composable morphisms $f, g$. Thus associating to a pair $(n, \alpha)$ the family of vector bundles $Y \mapsto \mathcal{P}_{n, f^{*}(\alpha)}$ we get a big vector bundle on $X$. Once again the natural functor from the category of small vector bundles to that of big vector bundles is an equivalence provided that the scheme $X$ is affine. Define the tensor product of two small 
vector bundles using the formula

$$
(n, \alpha) \otimes_{\mathcal{O}_{X}}(m, \beta)= \begin{cases}(n m, \alpha \otimes \beta), & \text { if } \alpha \otimes \beta \neq 0, \\ 0, & \text { if } \alpha \otimes \beta=0,\end{cases}
$$

where $(\alpha \otimes \beta)_{k, l}=\alpha_{i, j} \cdot \beta_{i^{\prime}, j^{\prime}}$ provided that $k=\left(i^{\prime}-1\right) n+i, l=\left(j^{\prime}-1\right) n+j(1 \leqslant i, j \leqslant n$; $\left.1 \leqslant i^{\prime}, j^{\prime} \leqslant m\right)$. Note that $f^{*}((n, \alpha) \otimes(m, \beta))=f^{*}(n, \alpha) \otimes f^{*}(m, \beta)$ so that for small vector bundles the tensor product is strictly functorial. Note also that the tensor product operation for small vector bundles is strictly associative.

Replacing big vector bundles by small ones in the definition of the prespectrum $\mathcal{K}^{\mathcal{Q}, \mathbb{A}^{q}}\left(X \times \Delta^{\bullet}\right)$, we get an $\Omega$ - prespectrum

$$
\left.\mathcal{K}_{s m}^{\mathcal{Q}, \mathbb{A}^{q}}\left(X \times \Delta^{\bullet}\right)=\left(w S_{\bullet} \mathcal{C} \mathcal{P}_{s m}^{\mathcal{Q}, \mathbb{A}^{q}}\left(X \times \Delta^{\bullet}\right)\right), w S_{\bullet} S_{\bullet} \mathcal{C} \mathcal{P}_{s m}^{\mathcal{Q}, \mathbb{A}^{q}}\left(X \times \Delta^{\bullet}\right), \ldots\right),
$$

where $\mathcal{C} \mathcal{P}_{s m}^{\mathcal{Q}, \mathbb{A}^{q}}(Y)$ stands for the category of bounded complexes of small vector bundles on $Y \times \mathbb{A}^{q}$ acyclic outside of the family of supports consisting of subschemes quasi-finite over $Y$. Moreover we have an obvious morphism of prespectra $\mathcal{K}_{s m}^{\mathcal{Q}, \mathbb{A}^{q}}(X) \rightarrow \mathcal{K}^{\mathcal{Q}, \mathbb{A}^{q}}(X)$ which is a weak equivalence in the case of affine schemes.

Let $X, X^{\prime} ; S, S^{\prime}$ be any smooth schemes. The tensor product operation on complexes of (small) vector bundles defines a functor

$$
\begin{aligned}
& \mathcal{C} \mathcal{P}_{s m}^{\mathcal{Q}, S}(X) \times \mathcal{C} \mathcal{P}_{s m}^{\mathcal{Q}, S^{\prime}}\left(X^{\prime}\right) \rightarrow \mathcal{C} \mathcal{P}_{s m}^{\mathcal{Q}, S \times S^{\prime}}\left(X \times X^{\prime}\right), \\
& C_{*} \times C_{*}^{\prime} \mapsto p^{*}\left(C_{*}\right) \otimes \mathcal{O}_{X \times X^{\prime} \times S \times S^{\prime}}\left(p^{\prime}\right)^{*}\left(C_{*}^{\prime}\right),
\end{aligned}
$$

where $X \times S \stackrel{p}{\leftarrow} X \times X^{\prime} \times S \times S^{\prime} \stackrel{p^{\prime}}{\rightarrow} X^{\prime} \times S^{\prime}$ are the canonical projections. This functor is exact in each variable and preserves weak equivalences. Thus it defines a pairing of simplicial sets (see Appendix C.2)

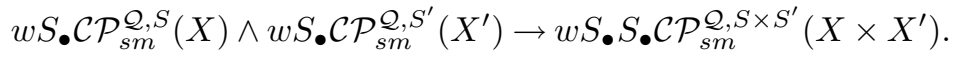

Composing this external pairing with the diagonal map we get internal pairings defined for any $X \in S m / F, S, S^{\prime} \in S m / F$

$$
\begin{aligned}
w S . \mathcal{C} \mathcal{P}_{s m}^{\mathcal{Q}, S}(X) \wedge w S_{\bullet} \mathcal{C} \mathcal{P}_{s m}^{\mathcal{Q}, S^{\prime}}(X) & \rightarrow w S \bullet S . \mathcal{C} \mathcal{P}_{s m}^{\mathcal{Q}, S \times S^{\prime}}(X \times X) \\
& \stackrel{\Delta^{*}}{\longrightarrow} w S_{\bullet} S_{\bullet} \mathcal{C} \mathcal{P}_{s m}^{\mathcal{Q}, S \times S^{\prime}}(X) .
\end{aligned}
$$

Since our tensor product operation is strictly functorial one checks immediately that the above construction generalizes to cosimplicial schemes, i.e., for any (smooth) cosimplicial scheme $X^{\bullet}$ we have a natural pairing

$$
w S_{\bullet} \mathcal{C} \mathcal{P}_{s m}^{\mathcal{Q}, S}\left(X^{\bullet}\right) \wedge w S_{\bullet} \mathcal{C} \mathcal{P}_{s m}^{\mathcal{Q}, S^{\prime}}\left(X^{\bullet}\right) \rightarrow w S \bullet S . \mathcal{C} \mathcal{P}_{s m}^{\mathcal{Q}, S \times S^{\prime}}\left(X^{\bullet}\right) .
$$

In particular we get canonical pairings

$$
w S_{\bullet} \mathcal{C} \mathcal{P}_{s m}^{\mathcal{Q}, \mathbb{A}^{q}}\left(X \times \Delta^{\bullet}\right) \wedge w S_{\bullet} \mathcal{C} \mathcal{P}_{s m}^{\mathcal{Q}, \mathbb{A}^{q^{\prime}}}\left(X \times \Delta^{\bullet}\right) \rightarrow w S_{\bullet} S_{\bullet} \mathcal{C} \mathcal{P}_{s m}^{\mathcal{Q}, \mathbb{A}^{q+q^{\prime}}}\left(X \times \Delta^{\bullet}\right) .
$$

Returning to the notations and assumptions of Section 13, let $X \in S m / F$ be a smooth scheme over a field $F$. For any integer $q \geqslant 0$ denote by $\Omega^{-1} \mathcal{K}_{s m}^{q}$ (respectively $\Omega^{-2} \mathcal{K}_{s m}^{q}$ ) the simplicial 
sheaf on $X$ associated to the presheaf $U \mapsto w S_{\bullet} \mathcal{C} \mathcal{P}_{s m}^{\mathcal{Q}, \mathbb{A}^{q}}\left(\Delta^{\bullet} \times U\right)$ (respectively

$$
\left.U \mapsto w S_{\bullet} S_{\bullet} \mathcal{C} \mathcal{P}_{s m}^{\mathcal{Q}, \mathbb{A}^{q}}\left(\Delta^{\bullet} \times U\right)\right)
$$

The previous discussion shows that the natural maps

$$
\Omega^{-1} \mathcal{K}_{s m}^{q} \rightarrow \Omega^{-1} \mathcal{K}^{q}, \quad \Omega^{-2} \mathcal{K}_{s m}^{q} \rightarrow \Omega^{-2} \mathcal{K}^{q}
$$

are weak equivalences and furthermore we have a natural pairing of simplicial sheaves $\Omega^{-1} \mathcal{K}_{s m}^{q} \wedge \Omega^{-1} \mathcal{K}_{s m}^{q^{\prime}} \rightarrow \Omega^{-2} \mathcal{K}_{s m}^{q+q^{\prime}}$

For the remainder of this section we only consider small vector bundles, and we shall drop the subscript $\mathrm{sm}$ in the notations; also, we shall always use the canonical flasque resolution $\mathcal{R}(L)$ when talking about the simplicial set $R \Gamma(U, L)$, i.e., set $R \Gamma(U, L)=\Gamma(U, \mathcal{R}(L))$ (cf. Proposition D.6).

Generalizing the previous discussion slightly we come to the following conclusion.

LEMma 15.1. - For any $X \in S m / F$, the tensor product pairings induce natural pairings of simplicial sheaves on $X$

$$
\Omega^{-i} \mathcal{K}^{q} \wedge \Omega^{-j} \mathcal{K}^{q^{\prime}} \rightarrow \Omega^{-(i+j)} \mathcal{K}^{q+q^{\prime}}
$$

and thereby pairings of connected spaces

$$
R \Gamma\left(X, \Omega^{-i} \mathcal{K}^{q}\right) \wedge R \Gamma\left(X, \Omega^{-j} \mathcal{K}^{q^{\prime}}\right) \rightarrow R \Gamma\left(X, \Omega^{-(i+j)} \mathcal{K}^{q+q^{\prime}}\right)
$$

We plan to verify that the pairings introduced above satisfy the conditions of Theorem 14.4 and hence induce products on the spectral sequence relating motivic cohomology to $K$-theory.

Note that for each $q$ we have $q+1$ coordinate embeddings $\mathbb{A}^{q} \stackrel{i_{0}, \ldots, i_{q}}{\longrightarrow} \mathbb{A}^{q+1}$ given by the formulas $i_{k}:\left(x_{1}, \ldots, x_{q}\right) \mapsto\left(x_{1}, \ldots, x_{k}, 0, x_{k+1}, \ldots, x_{q}\right)$, which satisfy the cosimplicial relations $i_{k} \circ i_{l}=i_{l} \circ i_{k-1}: \mathbb{A}^{q-1} \rightarrow \mathbb{A}^{q+1}(0 \leqslant l<k \leqslant q)$. Associated to these embeddings we have $q+1$ morphisms of simplicial prespectra $f_{0}=\left(i_{0}\right)^{*}, \ldots, f_{q}=\left(i_{q}\right)^{*}: \mathcal{K}^{\mathcal{Q}, \mathbb{A}^{q+1}} \rightarrow \mathcal{K}^{\mathcal{Q}, \mathbb{A}^{q}}$ which satisfy the corresponding simplicial relations. Note also that we chose the map $f=f_{q}: \mathcal{K}^{q+1} \rightarrow \mathcal{K}^{q}$ as the preferred one in the tower of fibrations defining the spectral sequence of Section 13.

LEMMA 15.2. - The tensor product pairings of Lemma 15.1 fit in commutative squares

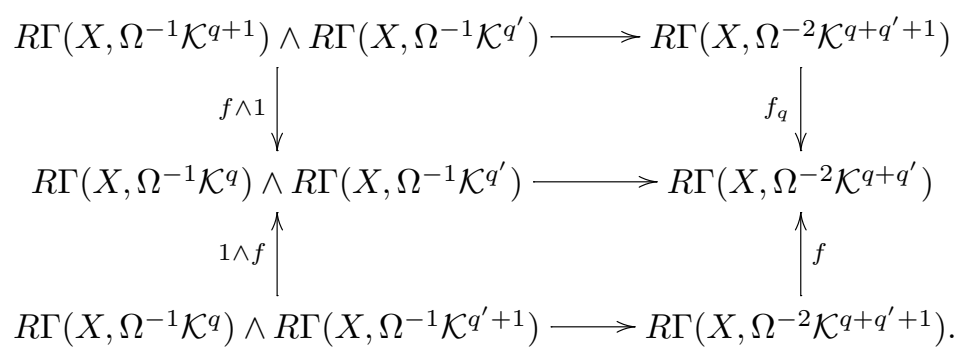


Proof. - This follows immediately from the commutativity of the following diagram of schemes

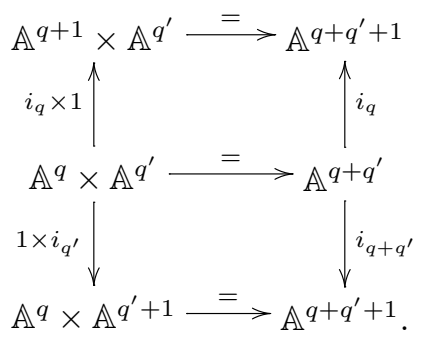

For any (affine) scheme $X$ the pairing of simplicial sets

$$
w S_{\bullet} \mathcal{C} \mathcal{P}^{\mathcal{Q}, \mathbb{A}^{q}}(X) \wedge w S_{\bullet} \mathcal{C P}{\mathcal{Q}, \mathbb{A}^{q^{\prime}}}^{(X) \rightarrow w S . S} \cdot \mathcal{C} \mathcal{P}^{\mathcal{Q}, S \times S^{\prime}}(X)
$$

induces a pairing on $K_{0}$-groups $K_{0}^{\mathcal{Q}, \mathbb{A}^{q}}(X) \otimes K_{0}^{\mathcal{Q}, \mathbb{A}^{q^{\prime}}}(X) \rightarrow K_{0}^{\mathcal{Q}, \mathbb{A}^{q+q^{\prime}}}(X)$ and it's clear from the definitions that the following diagram commutes

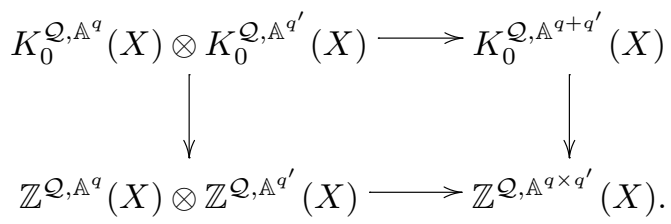

Here the vertical arrows are the canonical homomorphisms of Lemma 8.7, and the bottom pairing sends the pair of cycles $\left(Z, Z^{\prime}\right)$ to the cycle $\left(\Delta_{X}\right)^{*}\left(Z \times Z^{\prime}\right)$.

The pairings $\mathbb{Z}^{\mathcal{Q}, \mathbb{A}^{q}}\left(X \times \Delta^{n}\right) \otimes \mathbb{Z}^{\mathcal{Q}, \mathbb{A}^{q^{\prime}}}\left(X \times \Delta^{n}\right) \rightarrow \mathbb{Z}^{\mathcal{Q}, \mathbb{A}^{q \times q^{\prime}}}\left(X \times \Delta^{n}\right)$ determine a pairing of simplicial abelian sheaves $C_{\bullet}\left(\mathbb{Z}^{\mathcal{Q}, \mathbb{A}^{q}}\right) \otimes C_{\bullet}\left(\mathbb{Z}^{\mathcal{Q}, \mathbb{A}^{q^{\prime}}}\right) \rightarrow C_{\bullet}\left(\mathbb{Z}^{\mathcal{Q}, \mathbb{A}^{q \times q^{\prime}}}\right)$ and the induced pairing of classifying simplicial sheaves (see discussion after Lemma B.3)

$$
B\left(C \bullet\left(\mathbb{Z}^{\mathcal{Q}, \mathbb{A}^{q}}\right)\right) \otimes B\left(C \bullet\left(\mathbb{Z}^{\mathcal{Q}, \mathbb{A}^{q^{\prime}}}\right)\right) \rightarrow B^{2}\left(C \bullet\left(\mathbb{Z}^{\mathcal{Q}, \mathbb{A}^{q \times q^{\prime}}}\right)\right) .
$$

Thus we get, in particular, a pairing of simplicial sheaves

$$
\begin{aligned}
& B\left(C \bullet\left(\mathbb{Z}^{\mathcal{Q}, \mathbb{A}^{q}}\right)\right) \wedge B\left(C \bullet\left(\mathbb{Z}^{\mathcal{Q}, \mathbb{A}^{q^{\prime}}}\right)\right) \\
& \quad \rightarrow B\left(C_{\bullet}\left(\mathbb{Z}^{\mathcal{Q}, \mathbb{A}^{q}}\right)\right) \otimes B\left(C_{\bullet}\left(\mathbb{Z}^{\mathcal{Q}, \mathbb{A}^{q^{\prime}}}\right)\right) \rightarrow B^{2}\left(C_{\bullet}\left(\mathbb{Z}^{\mathcal{Q}, \mathbb{A}^{q \times q^{\prime}}}\right)\right) .
\end{aligned}
$$

Given the discussion, the following result is now immediate from the definitions.

LEMMA 15.3. - The following diagram of simplicial sheaves commutes

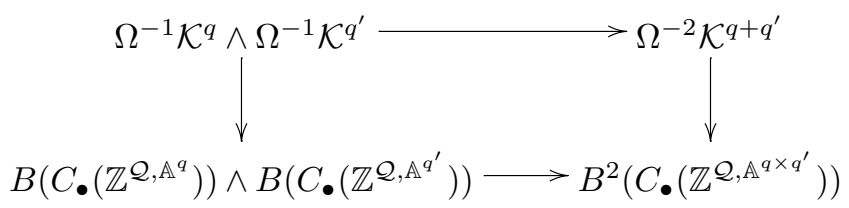


and hence determines the following diagram of simplicial sets (cf. Proposition D.6)

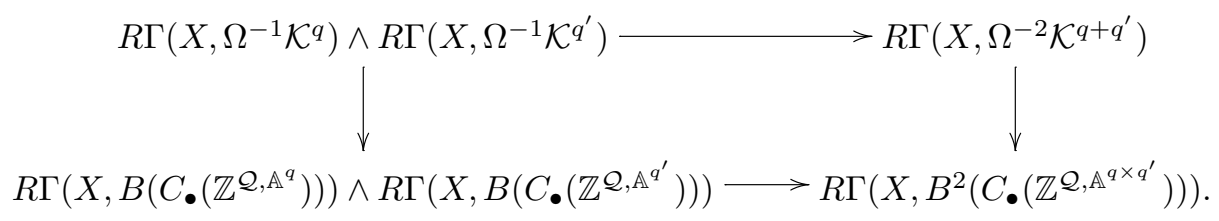

LEMMA 15.4. - The maps of simplicial sheaves

$$
\Omega^{-1} \mathcal{K}^{q+1} \underset{f_{q-1}}{\stackrel{f_{q}}{\longrightarrow}} \Omega^{-1} \mathcal{K}^{q}
$$

are related by a simplicial homotopy which becomes constant when composed with the maps $\Omega^{-1} \mathcal{K}^{q} \stackrel{f}{\rightarrow} \Omega^{-1} \mathcal{K}^{q-1}$ and $\Omega^{-1} \mathcal{K}^{q} \rightarrow B\left(C \cdot\left(\mathbb{Z}^{\mathcal{Q}, \mathbb{A}^{q}}\right)\right)$. Hence the same conclusion holds for the maps of simplicial sets

$$
R \Gamma\left(X, \Omega^{-1} \mathcal{K}^{q+1}\right) \underset{f_{q-1}}{\stackrel{f_{q}}{\longrightarrow}} R \Gamma\left(X, \Omega^{-1} \mathcal{K}^{q}\right) .
$$

Proof. - This follows immediately from Lemma 8.10, Lemma 8.10.2 and properties of the functor $\mathcal{R}$ described in Appendix D.

THEOREM 15.5. - The tensor product operation on complexes of (small) vector bundles induces a multiplicative structure on the spectral sequence of (13.6.1) (reindexed by changing signs)

$$
E_{p, q}^{2}=\pi_{p+q} R \Gamma\left(X, B\left(C \bullet\left(\mathbb{Z}^{\mathcal{Q}, \mathbb{A}^{q}}\right)\right)\right) \quad \Longrightarrow \quad K_{p+q}(X) .
$$

The product structure on the abutment $K_{*}(X)$ is the usual product structure on $K$-theory and the product structure on the $E_{2}$ term is the usual product structure in motivic cohomology (see Section 12).

Proof. - We have three towers of fibrations satisfying the conditions of Proposition 6.1:

$$
\begin{aligned}
& X_{q}^{\prime}=X_{q}^{\prime \prime}=R \Gamma\left(X, \Omega^{-1} \mathcal{K}^{q}\right), \quad X_{q}=R \Gamma\left(X, \Omega^{-2} \mathcal{K}^{q}\right), \\
& B_{q}^{\prime}=B_{q}^{\prime \prime}=R \Gamma\left(X, B\left(C \bullet\left(\mathbb{Z}^{\mathcal{Q}, \mathbb{A}^{q}}\right)\right)\right), \quad B_{q}=R \Gamma\left(X, B^{2}\left(C \bullet\left(\mathbb{Z}^{\mathcal{Q}, \mathbb{A}^{q}}\right)\right)\right) .
\end{aligned}
$$

Moreover we have pairings $X_{q}^{\prime} \wedge X_{q}^{\prime \prime} \rightarrow X_{q}, B_{q}^{\prime} \wedge B_{q}^{\prime \prime} \rightarrow B_{q}$. The discussion prior to Lemma 15.2 together with Lemmas 15.2, 15.3, 15.4 and 12.3 shows that the conditions of Theorem 14.4 (with $M^{\prime}=M^{\prime \prime}=M=S^{0}$ ) are satisfied and hence we get a pairing of the spectral sequences defined by $\left(X^{\prime}, B^{\prime}\right)$ and $\left(X^{\prime \prime}, B^{\prime \prime}\right)$ to the spectral sequence defined by $(X, B)$. However all three spectral sequences coincide (after appropriate re-indexing) by Proposition 13.11. Thus we get a multiplicative structure on our spectral sequence. The product structure on the limit is easily seen to coincide with the usual multiplicative structure on $K$-theory.

To identify the multiplicative structure on the $E_{2}$ term we need a few additional remarks before proving this in Corollary 15.5.4.

Remark 15.5.1. - Let $A_{\bullet}, B_{\bullet}$ and $C_{\bullet}$ be simplicial abelian sheaves. The data of a bilinear pairing of simplicial abelian sheaves $A_{\bullet} \times B_{\bullet} \rightarrow C_{\bullet}$ defines a homomorphism of complexes of abelian sheaves $A_{*} \otimes B_{*} \stackrel{E Z}{\longrightarrow} C_{*}$ where $E Z$ is obtained using the Eilenberg-Zilber 
homomorphism $A_{*} \otimes B_{*} \stackrel{E Z}{\longrightarrow}\left(A_{\bullet} \otimes B_{\bullet}\right)_{*}-$ see Appendix B.1. Moreover applying this construction to the pairing $C_{\bullet}\left(\mathbb{Z}^{\mathcal{Q}, \mathbb{A}^{q}}\right) \times C_{\bullet}\left(\mathbb{Z}^{\mathcal{Q}, \mathbb{A}^{q^{\prime}}}\right) \rightarrow C_{\bullet}\left(\mathbb{Z}^{\mathcal{Q}, \mathbb{A}^{q+q^{\prime}}}\right)$ we get (after re-indexing) the homomorphism $\mathbb{Z}(q) \otimes \mathbb{Z}\left(q^{\prime}\right) \rightarrow \mathbb{Z}\left(q+q^{\prime}\right)$ introduced in Section 12 which defines products in motivic cohomology.

Return back to the case of an arbitrary bilinear pairing of simplicial abelian sheaves

$$
A_{\bullet} \times B_{\bullet} \rightarrow C_{\bullet}
$$

and the associated homomorphism of complexes of abelian sheaves

$$
A^{*} \otimes B^{*} \stackrel{E Z}{\longrightarrow} C^{*} \text {. }
$$

Since the functor $\mathcal{R}$ preserves products we conclude immediately that the simplicial sheaves $\mathcal{R}\left(A_{\bullet}\right), \mathcal{R}\left(B_{\bullet}\right), \mathcal{R}\left(C_{\bullet}\right)$ are actually simplicial abelian sheaves and the augmentation maps $A_{\bullet} \stackrel{\phi_{A}}{\longrightarrow} \mathcal{R}\left(A_{\bullet}\right), \ldots$ are homomorphisms of simplicial abelian sheaves. Furthermore applying the functor $\mathcal{R}$ to the map $A_{\bullet} \times B_{\bullet} \rightarrow C_{\bullet}$ we get a map of simplicial sheaves

$$
\mathcal{R}\left(A_{\bullet}\right) \times \mathcal{R}\left(B_{\bullet}\right)=\mathcal{R}\left(A_{\bullet} \times B_{\bullet}\right) \rightarrow \mathcal{R}\left(C_{\bullet}\right)
$$

which is easily seen to be bilinear. Taking finally global sections we get a bilinear pairing of simplicial abelian groups

$$
R \Gamma\left(X, A_{\bullet}\right) \times R \Gamma\left(X, B_{\bullet}\right) \rightarrow R \Gamma\left(X, C_{\bullet}\right)
$$

and hence the induced pairing in homotopy groups

$$
\pi_{p}\left(R \Gamma\left(X, A_{\bullet}\right)\right) \otimes \pi_{q}\left(R \Gamma\left(X, B_{\bullet}\right)\right) \rightarrow \pi_{p+q}\left(R \Gamma\left(X, C_{\bullet}\right)\right) .
$$

LEMMA 15.5.2. - After the identification of $\pi_{p}\left(R \Gamma\left(X, A_{\bullet}\right)\right)$ with $H^{-p}\left(X, A^{*}\right)$, $\pi_{q}\left(R \Gamma\left(X, B_{\bullet}\right)\right)$ with $H^{-q}\left(X, B^{*}\right)$ and $\pi_{p+q}\left(R \Gamma\left(X, C_{\bullet}\right)\right)$ with $H^{-p-q}\left(X, C^{*}\right)$ (see Corollary 13.3.1) the above pairing in homotopy groups coincides with the pairing

$$
H^{-p}\left(X, A^{*}\right) \otimes H^{-q}\left(X, B^{*}\right) \rightarrow H^{-p-q}\left(X, C^{*}\right)
$$

defined by the homomorphism of complexes $A^{*} \otimes B^{*} \rightarrow C^{*}$.

Proof. - The pairing in homotopy groups defined by the bilinear pairing

$$
R \Gamma\left(X, A_{\bullet}\right) \times R \Gamma\left(X, B_{\bullet}\right) \rightarrow R \Gamma\left(X, C_{\bullet}\right)
$$

coincides with the pairing in homology defined by the homomorphism of complexes (see Proposition B.2)

$$
\begin{aligned}
\Gamma\left(X, \mathcal{R}\left(A_{\bullet}\right)\right)^{*} \otimes \Gamma\left(X, \mathcal{R}\left(B_{\bullet}\right)\right)^{*} & =R \Gamma\left(X, A_{\bullet}\right)^{*} \otimes R \Gamma\left(X, B_{\bullet}\right)^{*} \rightarrow \Gamma\left(X, \mathcal{R}\left(C_{\bullet}\right)\right)^{*} \\
& =R \Gamma\left(X, C_{\bullet}\right)^{*}
\end{aligned}
$$

Furthermore the homomorphisms $A^{*} \stackrel{\phi_{A}}{\longrightarrow} \mathcal{R}\left(A_{\bullet}\right)^{*}, \ldots$ are quasi-isomorphisms and hence define canonical maps

$$
H^{p}\left(\Gamma\left(X, \mathcal{R}\left(A_{\bullet}\right)\right)\right) \rightarrow H^{p}\left(X, A^{*}\right)
$$


which are isomorphisms in non-positive degrees according to Corollary A.4. Finally we have a commutative diagram of complexes of sheaves

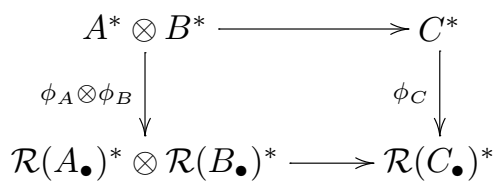

which according to Theorem A.6 yields a commutative diagram in cohomology

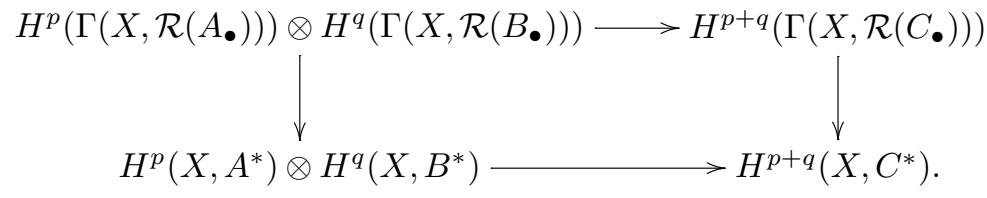

Here the bottom arrow is the product pairing in hypercohomology defined by the homomorphism of complexes of sheaves $A^{*} \otimes B^{*} \rightarrow C^{*}$, the top arrow coincides with the pairing in homotopy groups we are studying and the vertical arrows are isomorphisms provided that both $p$ and $q$ are non-positive.

Lemma 15.5.2 admits the following generalization which together with Remark 15.5.1 completes the proof of the Theorem 15.5.

LEMMA 15.5.3. - For any simplicial abelian sheaf $A \bullet$ on a Noetherian topological space of finite Krull dimension we have natural identifications

$$
\pi_{i}\left(R \Gamma\left(X, B^{k} A_{\bullet}\right)\right)=H^{-i+k}\left(X, A^{*}\right) \quad i \geqslant 0 .
$$

Moreover given a bilinear pairing of simplicial abelian sheaves $A_{\bullet} \times B_{\bullet} \rightarrow C_{\bullet}$, the pairing in homotopy groups defined by the bilinear pairing of simplicial abelian groups

$$
R \Gamma\left(X, B^{k} A_{\bullet}\right) \times R \Gamma\left(X, B^{k^{\prime}} B_{\bullet}\right) \rightarrow R \Gamma\left(X, B^{k+k^{\prime}} C_{\bullet}\right)
$$

coincides with the pairing in the hypercohomology groups induced by the pairing of complexes $A^{*} \otimes B^{*} \rightarrow C^{*}$.

Proof. - The homotopy group $\pi_{p}\left(R \Gamma\left(X, B^{k} A_{\bullet}\right)\right)$ coincides with $H^{-p}\left(X,\left(B^{k} A_{\bullet}\right)^{*}\right)$. Furthermore the complex $\left(B^{k} A_{\bullet}\right)^{*}$ is canonically quasi-isomorphic to $A^{*}[k]$ (using the usual explicit form of the Eilenberg-Zilber map given in Lemma B.3) and hence

$$
H^{-p}\left(X,\left(B^{k} A_{\bullet}\right)^{*}\right)=H^{-p+k}\left(X, A^{*}\right),
$$

which proves the first statement. To prove the second one it suffices to establish the commutativity of the following diagram (where the vertical arrows are the canonical quasi-isomorphisms introduced in Lemma B.3)

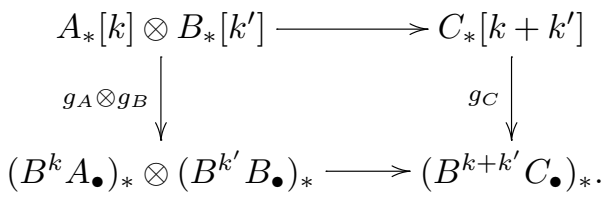

This is done by induction on $k$ and $k^{\prime}$ starting with Lemma B.4. 
COROLLARY 15.5.4. - For any $k, l \geqslant 0$ the pairing in homotopy groups induced by the bilinear pairing of simplicial abelian groups

$$
R \Gamma\left(X, B^{k}\left(C \bullet\left(\mathbb{Z}^{\mathcal{Q}, \mathbb{A}^{q}}\right)\right)\right) \times R \Gamma\left(X, B^{l}\left(C \bullet\left(\mathbb{Z}^{\mathcal{Q}, \mathbb{A}^{q^{\prime}}}\right)\right)\right) \rightarrow R \Gamma\left(X, B^{k+l}\left(C \bullet\left(\mathbb{Z}^{\mathcal{Q}, \mathbb{A}^{q+q^{\prime}}}\right)\right)\right)
$$

coincides (after re-indexing) with the product map in motivic cohomology.

\section{The spectral sequence for $K$-theory with finite coefficients}

In this final section, we discuss the natural version of our motivic spectral sequence (13.6.1) for $K$-theory with finite coefficients (cf. Appendix C.3). In particular, we discuss the multiplicative structure on the spectral sequence (16.2.1).

We recall the following facts concerning the existence and uniqueness of stable comultiplications on the mod-1 Moore space $M_{l}^{2}$.

PROPOSITION 16.1 (cf. [8,22]). - If $l$ is odd, then up to homotopy there is a unique comultiplication

$$
\Sigma^{2} M_{l}^{2} \stackrel{c}{\rightarrow} M_{l}^{2} \wedge M_{l}^{2}
$$

which is homotopy inverse on the right to the canonical map $M_{l}^{2} \wedge M_{l}^{2} \stackrel{p \wedge 1_{M_{l}^{2}}}{\longrightarrow} \Sigma^{2} M_{l}^{2}$.

If $4 \mid l$, then such a comultiplication exists; there are 4 distinct stable homotopy classes of such maps $M_{l} \wedge M_{l} \rightarrow M_{l}$. If $8 \mid l$, then only two of these have coboundary which is a derivation and these two differ by the adjoint of $\eta^{2}: M_{l} \wedge M_{l} \rightarrow S^{1} \wedge S^{1} \rightarrow S^{0} \rightarrow M_{l}$.

THEOREM 16.2. - As in Theorem 13.6, the tower of spaces

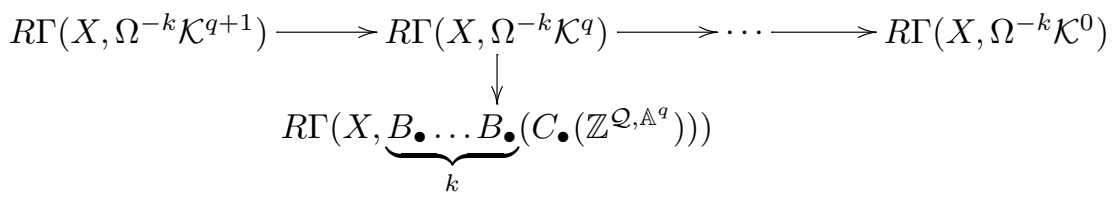

yields a strongly convergent spectral sequence for the $K$-theory with coefficients $\mathbb{Z} / l$

$$
E_{2}^{p, q}=H^{p-q}(X, \mathbb{Z} / l(-q)) \quad \Rightarrow \quad K_{-p-q}(X, \mathbb{Z} / l)
$$

which is independent of the choice of $k \geqslant 3$.

Moreover, in case $l \not \equiv 2 \bmod 4$, a choice of comultiplication $\Sigma^{2} M_{l}^{2} \stackrel{c}{\rightarrow} M_{l}^{2} \wedge M_{l}^{2}$ as in Proposition 16.1 determines a multiplicative structure on this spectral sequence. On the limit, this multiplicative structure coincides with the multiplicative structure on mod-l $K$-theory using the given comultiplication and on the $E_{2}$-term coincides with the multiplicative structure on the motivic cohomology with finite coefficients (and in particular is independent of the choice of c).

Finally for any $l$ we have a canonical pairing between the integral spectral sequence and the spectral sequence with coefficients $\mathbb{Z} / l$.

Proof. - The existence of the spectral sequence follows from Proposition 14.2 and its independence of the choice of $k \geqslant 3$ follows by Corollary 6.1.1 from the fact that $R \Gamma\left(X, \mathcal{K}^{q}\right)$ and $R \Gamma\left(X, \mathbb{B}\left(C_{\bullet}\left(\mathbb{Z}^{\mathcal{Q}, \mathbb{A}^{q}}\right)\right)\right)$ are $((-1)$-connected $) \Omega$-prespectra.

For any $k, k^{\prime} \geqslant 3$ we have a natural pairing of the tower determined by the simplicial sheaves $\Omega^{-k} \mathcal{K}^{q}$ and the tower determined by the simplicial sheaves $\Omega^{-k^{\prime}} \mathcal{K}^{q^{\prime}}$ to the tower determined by 
the simplicial sheaves $\Omega^{-k-k^{\prime}} \mathcal{K}^{q+q^{\prime}}$. Moreover, as we saw in Section 15 , the other conditions of Theorem 14.4 are satisfied. Thus, taking $M^{\prime}=M^{\prime \prime}=M=M_{l}^{2}$ we get a natural multiplicative structure on our spectral sequence (which is also independent of the choice of $k, k^{\prime}$ ). The multiplicative structure on the limit obviously coincides with the usual multiplicative structure on the $K$-theory with coefficients $\mathbb{Z} / l$.

Thus, once again, the only thing to verify is that the multiplicative structure on the $E_{2}$-term coincides with the usual multiplicative structure on motivic cohomology with finite coefficients (and, in particular, is independent of the choice of comultiplication on the space $M_{l}^{2}$ ). This is done in essentially the same way as in the proof of Theorem 15.5. We sketch the main steps below.

Lemma 16.3. - Let A. be a simplicial abelian sheaf on a Noetherian topological space of finite Krull dimension. Then for any $p \geqslant 1$,

$$
\pi_{p}\left(R \Gamma\left(X, A_{\bullet}\right), \mathbb{Z} / l\right)=H^{-p}\left(X, C_{l}\left(A^{*}\right)\right)
$$

where $C_{l}\left(A^{*}\right)=C\left(A^{*} \stackrel{l}{\rightarrow} A^{*}\right)$ is the cone of the multiplication by $l$ on $A^{*}$. If the abelian sheaves $A_{i}$ are $\mathbb{Z}$-flat, the above cohomology group may be further identified with $H^{-p}\left(X, A^{*} / l\right)$

Proof. - According to Lemma C.3.1, we have a natural identification

$$
\pi_{p}\left(R \Gamma\left(X, A_{\bullet}\right), \mathbb{Z} / l\right)=H_{p}\left(C_{l}\left(R \Gamma\left(X, A_{\bullet}\right)\right)\right)=H^{-p}\left(\Gamma\left(X, C_{l}\left(\mathcal{R}\left(A_{\bullet}\right)^{*}\right)\right)\right) .
$$

As seen in Appendix A, the quasi-isomorphism $C_{l}\left(A^{*}\right) \rightarrow C_{l}\left(\mathcal{R}\left(A_{\bullet}\right)^{*}\right)$ gives us canonical homomorphisms $H^{-p}\left(\Gamma\left(X, C_{l}\left(\mathcal{R}\left(A_{\bullet}\right)^{*}\right)\right) \rightarrow H^{-p}\left(X, C_{l}\left(A^{*}\right)\right)\right.$. Moreover, since all terms of the complex $C_{l}\left(\mathcal{R}\left(A_{\bullet}\right)^{*}\right)$ except possibly for the ones in degrees 0 and -1 are flasque sheaves, the above homomorphisms are isomorphisms in degrees $p \geqslant 1-$ see Corollary A.4.

The last part of the statement is obvious since for complexes of $\mathbb{Z}$-flat sheaves the natural map $C_{l}\left(A^{*}\right) \rightarrow A^{*} / l$ is a quasi-isomorphism.

Let $A_{\bullet} \times B_{\bullet} \rightarrow C_{\bullet}$ be a bilinear pairing of simplicial abelian sheaves on a Noetherian topological space $X$ of finite Krull dimension. This pairing induces a bilinear pairing of simplicial abelian groups (see the discussion after Remark 15.5.1)

$$
R \Gamma\left(X, A_{\bullet}\right) \times R \Gamma\left(X, B_{\bullet}\right) \rightarrow R \Gamma\left(X, C_{\bullet}\right),
$$

and hence an associated pairing in homotopy groups with finite coefficients

$$
\pi_{i}\left(R \Gamma\left(X, A_{\bullet}\right), \mathbb{Z} / l\right) \otimes \pi_{j}\left(R \Gamma\left(X, B_{\bullet}\right), \mathbb{Z} / l\right) \rightarrow \pi_{i+j}\left(R \Gamma\left(X, C_{\bullet}\right), \mathbb{Z} / l\right) .
$$

On the other hand the pairing $A_{\bullet} \times B_{\bullet} \rightarrow C_{\bullet}$ determines a pairing of complexes of sheaves $A^{*} \otimes B^{*} \rightarrow C^{*}$ (see Appendix B) and hence also a pairing $C_{l}\left(A^{*}\right) \otimes C_{l}\left(B^{*}\right) \rightarrow C_{l}\left(C^{*}\right)$. The last pairing determines further a pairing in hypercohomology groups

$$
H^{-i}\left(X, C_{l}\left(A^{*}\right)\right) \otimes H^{-j}\left(X, C_{l}\left(B^{*}\right)\right) \rightarrow H^{-i-j}\left(X, C_{l}\left(C^{*}\right)\right) .
$$

LEMma 16.4. - After the identification of $\pi_{i}\left(R \Gamma\left(X, A_{\bullet}\right), \mathbb{Z} / l\right)$ with $H^{-i}\left(X, C_{l}\left(A^{*}\right)\right)$, $\pi_{j}\left(R \Gamma\left(X, B_{\bullet}\right), \mathbb{Z} / l\right)$ with $H^{-j}\left(X, C_{l}\left(B^{*}\right)\right)$, and $\pi_{i+j}\left(R \Gamma\left(X, C_{\bullet}\right), \mathbb{Z} / l\right)$ with $H^{-i-j}\left(X, C_{l}\left(C^{*}\right)\right)$ the pairing in homotopy groups

$$
\pi_{i}\left(R \Gamma\left(X, A_{\bullet}\right), \mathbb{Z} / l\right) \otimes \pi_{j}\left(R \Gamma\left(X, B_{\bullet}\right), \mathbb{Z} / l\right) \rightarrow \pi_{i+j}\left(R \Gamma\left(X, C_{\bullet}\right), \mathbb{Z} / l\right)
$$


coincides with the pairing in the hypercohomology groups

$$
H^{-i}\left(X, C_{l}\left(A^{*}\right)\right) \otimes H^{-j}\left(X, C_{l}\left(B^{*}\right)\right) \rightarrow H^{-i-j}\left(X, C_{l}\left(C^{*}\right)\right) .
$$

Proof. - The proof is essentially identical to that of Lemma 15.5.2; we leave the details to the reader.

The bilinear pairing of simplicial abelian sheaves

$$
C \bullet\left(\mathbb{Z}^{\mathcal{Q}, \mathbb{A}^{q}}\right) \times C_{\bullet}\left(\mathbb{Z}^{\mathcal{Q}, \mathbb{A}^{q^{\prime}}}\right) \rightarrow C_{\bullet}\left(\mathbb{Z}^{\mathcal{Q}, \mathbb{A}^{q+q^{\prime}}}\right)
$$

determines a homomorphism of complexes of sheaves

$$
C^{*}\left(\mathbb{Z}^{\mathcal{Q}, \mathbb{A}^{q}}\right) \otimes C^{*}\left(\mathbb{Z}^{\mathcal{Q}, \mathbb{A}^{q^{\prime}}}\right) \rightarrow C^{*}\left(\mathbb{Z}^{\mathcal{Q}, \mathbb{A}^{q+q^{\prime}}}\right)
$$

which up to re-indexing coincides with the pairing $\mathbb{Z}(q) \otimes \mathbb{Z}\left(q^{\prime}\right)$ introduced in Section 12 . According to Lemma 16.4, the pairing in homotopy groups

$$
\begin{aligned}
& \pi_{i}\left(R \Gamma\left(X, C \bullet\left(\mathbb{Z}^{\mathcal{Q}, \mathbb{A}^{q}}\right)\right), \mathbb{Z} / l\right) \otimes \pi_{j}\left(R \Gamma\left(X, C \bullet\left(\mathbb{Z}^{\mathcal{Q}, \mathbb{A}^{q^{\prime}}}\right)\right), \mathbb{Z} / l\right) \\
& \quad \rightarrow \pi_{i+j}\left(R \Gamma\left(X, C_{\bullet}\left(\mathbb{Z}^{\mathcal{Q}, \mathbb{A}^{q+q^{\prime}}}\right)\right), \mathbb{Z} / l\right)
\end{aligned}
$$

coincides with the pairing in hypercohomology groups induced by the homomorphism of complexes

$$
C_{l}\left(C^{*}\left(\mathbb{Z}^{\mathcal{Q}, \mathbb{A}^{q}}\right)\right) \otimes C_{l}\left(C^{*}\left(\mathbb{Z}^{\mathcal{Q}, \mathbb{A}^{q^{\prime}}}\right)\right) \rightarrow C_{l}\left(C^{*}\left(\mathbb{Z}^{\mathcal{Q}, \mathbb{A}^{q+q^{\prime}}}\right)\right)
$$

and hence coincides (up to reindexing) with the product map in motivic cohomology with finite coefficients.

To finish the proof of Theorem 16.2 it suffices now to establish the following lemma.

LEMMA 16.5. - For any simplicial abelian sheaf $A_{\bullet}$ on a Noetherian topological space of finite Krull dimension we have natural identifications

$$
\pi_{i}\left(R \Gamma\left(X, B^{k} A_{\bullet}\right), \mathbb{Z} / l\right)=H^{-i+k}\left(X, C_{l}\left(A^{*}\right)\right), \quad i \geqslant 2 .
$$

Moreover given a bilinear pairing of simplicial abelian sheaves $A_{\bullet} \times B_{\bullet} \rightarrow C_{\bullet}$, the pairing in homotopy groups with $\bmod l$ coefficients (with $l \not \equiv 2(\bmod 4))$ defined by the bilinear pairing of simplicial abelian groups

$$
R \Gamma\left(X, B^{k} A_{\bullet}\right) \times R \Gamma\left(X, B^{k^{\prime}} B_{\bullet}\right) \rightarrow R \Gamma\left(X, B^{k+k^{\prime}} C_{\bullet}\right)
$$

coincides with the pairing in hypercohomology groups induced by the pairing of complexes $C_{l}\left(A^{*}\right) \otimes C_{l}\left(B^{*}\right) \rightarrow C_{l}\left(C^{*}\right)$.

Proof. - The proof is identical to the proof of Lemma 15.5.3.

\section{Appendix A. Products in hypercohomology}

The purpose of this appendix is to exhibit multiplicative pairings in hypercohomology of not necessarily bounded complexes of sheaves on a Noetherian scheme of finite Krull dimension. Since there are certain pitfalls one must avoid, we present the construction here. 
The easiest way to define these multiplicative pairings is via the Godement resolutions (cf. [11], Chapter II.6.4). Recall that for an abelian sheaf $A$ on an arbitrary topological space $X$ its Godement resolution $G^{*}(A)$ is defined as follows. One first defines a sheaf $G(A)$ via the formula

$$
\Gamma(U, G(A))=\prod_{x \in U} A_{x}
$$

In other words $G(A)=\prod_{x \in X}\left(i_{x}\right)_{*}\left(A_{x}\right)$. Note that the sheaf $G(A)$ is obviously flasque. Furthermore we have a natural sheaf homomorphism $A \stackrel{i_{A}}{\longrightarrow} G(A)$ and the induced homomorphisms on stalks $A_{x} \rightarrow G(A)_{x}$ are canonically split by means of obvious maps $p_{x}: G(A)_{x} \rightarrow A_{x}$. Note also that the family of homomorphisms $p_{x}$ defines a sheaf homomorphism $p: G(G(A)) \rightarrow G(A)$.

Finally we set

$$
G^{n}(A)=\underbrace{G \circ \cdots \circ G}_{n+1}(A)
$$

(so that $G(A)=G^{0}(A)$ ) and define the coface operations

$$
\delta^{i}: G^{n}(A) \rightarrow G^{n+1}(A) \quad(0 \leqslant i \leqslant n+1)
$$

via the formulas

$$
G^{n}(A)=G^{i-1}\left(G^{n-i}(A)\right) \stackrel{G^{i-1}\left(i_{G^{n-i}(A)}\right)}{\longrightarrow} G^{i-1}\left(G^{n-i+1}(A)\right)=G^{n+1}(A) .
$$

The codegeneracy operators are defined similarly using now the homomorphisms $p$. One checks easily (see [11] Chapter II, §6) that the augmented cosimplicial sheaf $A \stackrel{i_{A}}{\longrightarrow} G^{*}(A)$ is acyclic, i.e., $G^{*}(A)$ is a flasque resolution of $A$. Moreover the homomorphism $i_{A}$ is a stalk-wise homotopy equivalence by $[11,4.3 .1]$. Here, and below, "homotopy equivalence" means chain homotopy equivalence of complexes.

If $B^{*, *}$ is a bicomplex of abelian sheaves, then we denote by

$$
\operatorname{Tot}\left(B^{*, *}\right), \quad \operatorname{Tot}\left(B^{*, *}\right)^{n}=\bigoplus_{s+t=n} B^{s, t}
$$

the associated total complex of sheaves. Note that we use direct sum and not direct product operation to define $\operatorname{Tot}\left(B^{*, *}\right)$. For example, we consider the total complex $\operatorname{Tot}\left(G^{*} A^{*}\right)$ of the bicomplex given by the Godement resolution of a complex $A^{*}$ of abelian sheaves. We observe that the natural homomorphism

$$
A^{*} \stackrel{i_{A^{*}}}{\longrightarrow} \operatorname{Tot}\left(G^{*} A^{*}\right)
$$

is a stalkwise homotopy equivalence for any complex $A^{*}$ of abelian sheaves: each $A^{j} \rightarrow G^{*}\left(A^{j}\right)$ is a homotopy equivalence of cosimplicial sheaves with natural homotopies compatible with respect to $A^{j} \rightarrow A^{j+1}$. In particular, $A^{*} \stackrel{i_{A^{*}}}{\longrightarrow} \operatorname{Tot}\left(G^{*} A^{*}\right)$ a quasi-isomorphism. Moreover the complex $\operatorname{Tot}\left(G^{*} A^{*}\right)$ consists of flasque sheaves provided that $A^{*}$ is bounded below or the topological space $X$ is Noetherian (in which case, an arbitrary direct sum of flasque sheaves is flasque).

Assume now that $X$ is a Noetherian topological space of finite Krull dimension. In this case we can use the following elementary but useful result.

Proposition A.1. - Let $A^{*}$ be an acyclic complex of abelian sheaves on a Noetherian topological space of finite Krull dimension. Assume that the sheaves $A^{i}$ are acyclic (e.g. flasque) for $i \leqslant n-1$. Then the complex $\Gamma\left(X, A^{*}\right)$ is acyclic in degrees $\leqslant n+1$. 
Proof. - Set $\mathcal{Z}^{i}=\operatorname{Ker}\left(A^{i} \stackrel{d}{\rightarrow} A^{i+1}\right)$. Using the short exact sequences

$$
0 \rightarrow \mathcal{Z}^{i-1} \rightarrow A^{i-1} \rightarrow \mathcal{Z}^{i} \rightarrow 0
$$

and acyclicity of $A^{i-1}$ for $0<i \leqslant n$ we conclude immediately that for $0<i \leqslant n$ we have isomorphisms

$$
H^{p}\left(X, \mathcal{Z}^{i}\right)=H^{p+1}\left(X, \mathcal{Z}^{i-1}\right)=\cdots=0
$$

since the cohomological dimension of abelian sheaves on $X$ is bounded by $\operatorname{dim} X$. Thus the sheaves $\mathcal{Z}^{i}(i \leqslant n)$ are also acyclic. This implies exactness of the following sequences

$$
\begin{aligned}
0 \rightarrow \Gamma\left(X, \mathcal{Z}^{n-1}\right) & \rightarrow \Gamma\left(X, A^{n-1}\right) \rightarrow \Gamma\left(X, \mathcal{Z}^{n}\right) \rightarrow 0, \\
0 \rightarrow \Gamma\left(X, \mathcal{Z}^{n}\right) & \rightarrow \Gamma\left(X, A^{n}\right) \rightarrow \Gamma\left(X, \mathcal{Z}^{n+1}\right) \rightarrow 0, \\
0 \rightarrow \Gamma\left(X, \mathcal{Z}^{n+1}\right) & \rightarrow \Gamma\left(X, A^{n+1}\right) \rightarrow \Gamma\left(X, A^{n+2}\right)
\end{aligned}
$$

and hence exactness of $\Gamma\left(X, A^{*}\right)$ in degrees $\leqslant n+1$.

COROllary A.2. - Let $A^{*} \rightarrow B^{*}$ be a quasi-isomorphism of complexes of abelian sheaves on a Noetherian topological space of finite Krull dimension. Assume further that the sheaves $A^{i}$ and $B^{i}$ are acyclic for $i \leqslant n-1$. Then the homomorphism of complexes of global sections $\Gamma\left(X, A^{*}\right) \rightarrow \Gamma\left(X, B^{*}\right)$ induces isomorphisms in cohomology in degrees $\leqslant n$.

Since the terms of the Godement resolution are flasque, Corollary A.2 justifies the following definition of the hypercohomology of $X$ with coefficients in an arbitrary (not necessarily bounded below) complex of sheaves $A^{*}$.

Definition A.3. - Let $A^{*}$ be a complex of abelian sheaves on a Noetherian topological space of finite Krull dimension. We define the hypercohomology of $X$ with coefficients in $A^{*}$ by the formula

$$
H^{p}\left(X, A^{*}\right)=H^{p}\left(\Gamma\left(X, \operatorname{Tot}\left(G^{*} A^{*}\right)\right)\right)=H^{p}\left(\operatorname{Tot}\left(\Gamma\left(X, G^{*} A^{*}\right)\right)\right) .
$$

One verifies easily that all the usual properties of hypercohomology still hold. In particular for any quasi-isomorphism $A^{*} \rightarrow \widetilde{A}^{*}$ we get canonical homomorphisms

$$
H^{p}\left(\Gamma\left(X, \widetilde{A}^{*}\right)\right) \rightarrow H^{p}\left(X, A^{*}\right)
$$

which arise from the commutative diagram of complexes

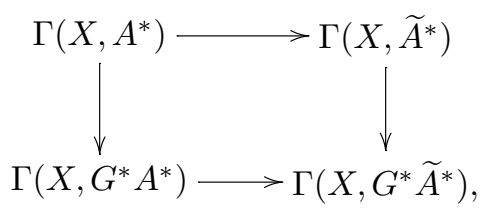

the bottom row of which is a quasi-isomorphism.

Consequently, Corollary A.2 immediately yields the following result.

COROLlary A.4. - Let $A^{*} \rightarrow \widetilde{A}^{*}$ be a quasi-isomorphism of complexes of abelian sheaves on a Noetherian topological space of finite Krull dimension. Assume that the sheaves $\widetilde{A}^{i}$ are acyclic for $i \leqslant n-1$. Then the associated homomorphisms $H^{p}\left(\Gamma\left(X, \widetilde{A}^{*}\right)\right) \rightarrow H^{p}\left(X, A^{*}\right)$ are isomorphisms in degrees $p \leqslant n$. 
Corollary A.4 shows in particular that hypercohomology may be equally defined using the Cartan-Eilenberg resolutions and hence we have the usual strongly convergent hypercohomology spectral sequences - see [38, 5.7.10].

The following lemma often simplifies the computations. We say that a homomorphism $A^{*} \rightarrow \widetilde{A}^{*}$ is a resolution of $A^{*}$ if it is a quasi-isomorphism.

LEMMA A.5 (cf. [11, II, 4.8.1]). - Let $A^{*} \rightarrow \widetilde{A}^{*}$ be a resolution of the complex $A^{*}$ of abelian sheaves. Assume further that we are given a homomorphism of resolutions, $f: \widetilde{A}^{*} \rightarrow G^{*} A^{*}$. Then the natural homomorphisms $H^{*}\left(\Gamma\left(X, \widetilde{A}^{*}\right)\right) \rightarrow H^{*}\left(X, A^{*}\right)=H^{*}\left(\Gamma\left(X, G^{*} A^{*}\right)\right)$ coincide with the homomorphisms induced by $f$.

Proof. - The general case is reduced easily to the case when $\widetilde{A}^{*}=G^{*} A^{*}$ and $f=I d$. In this case we have to show that the following two homomorphisms of complexes

$$
G^{*} A^{*} \underset{i_{G^{*} A^{*}}}{\stackrel{G^{*}\left(i_{A^{*}}\right)}{\longrightarrow}} G^{*} G^{*} A^{*}
$$

induce the same homomorphisms on homology of global sections. To do so we note that the homomorphism $G^{*}\left(i_{A^{*}}\right)-i_{G^{*} A^{*}}$ is killed on the right by a quasi-isomorphism $i_{A^{*}}$ and hence is killed on the left by an appropriate quasi-isomorphism $G^{*} G^{*} A^{*} \stackrel{g}{\rightarrow} B^{*}$. Replacing $B^{*}$ by its flasque resolution we may even assume that the complex $B^{*}$ consists of flasque sheaves. However in the latter case the homomorphism $\Gamma(X, g)$ of complexes of global sections is a quasi-isomorphism and our statement becomes obvious.

Assume now that $A^{*}$ and $B^{*}$ are two complexes of abelian sheaves on a Noetherian topological space $X$ of finite Krull dimension. The homomorphism $A^{*} \otimes_{\mathbb{Z}} B^{*} \stackrel{i_{A} \otimes i_{B}}{\longrightarrow} G^{*} A^{*} \otimes_{\mathbb{Z}} G^{*} B^{*}$ stalkwise is a tensor product of two homotopy equivalences and hence is a stalkwise homotopy equivalence as well. In particular $i_{A} \otimes_{\mathbb{Z}} i_{B}$ is a quasi-isomorphism. This gives us canonical pairings

$$
\begin{aligned}
H^{p}\left(X, A^{*}\right) \otimes H^{q}\left(X, B^{*}\right) & =H^{p}\left(\Gamma\left(X, G^{*} A^{*}\right)\right) \otimes H^{q}\left(\Gamma\left(X, G^{*} B^{*}\right)\right) \\
& \rightarrow H^{p+q}\left(\Gamma\left(X, G^{*} A^{*} \otimes_{\mathbb{Z}} G^{*} B^{*}\right)\right) \rightarrow H^{p+q}\left(X, A^{*} \otimes_{\mathbb{Z}} B^{*}\right) .
\end{aligned}
$$

Moreover we have a natural homomorphism of resolutions $G^{*} A^{*} \otimes_{\mathbb{Z}} G^{*} B^{*} \stackrel{f_{A, B}}{\longrightarrow} G^{*}\left(A^{*} \otimes_{\mathbb{Z}} B^{*}\right)$ - see [11] Chapter II, §6.4. Lemma A.5 shows now that the last arrow above coincides with the homomorphism in homology of global sections induced by $f_{A, B}$.

We now can formulate the naturality of pairings on hypercohomology. In the special case in which $A^{*}$ and $B^{*}$ are single sheaves, this is proved in [11], Theorem 6.6.1. We omit the proof of the general case stated below, for it presents no new difficulties.

THEOREM A.6. - Let $\widetilde{A}^{*}, \widetilde{B}^{*}, \widetilde{C}^{*}$ be resolutions of complexes $A^{*}, B^{*}$ and $C^{*}$ respectively. Assume further that we are given homomorphisms $A^{*} \otimes B^{*} \rightarrow C^{*}, \widetilde{A}^{*} \otimes \widetilde{B}^{*} \rightarrow \widetilde{C}^{*}$ which fit into a commutative diagram

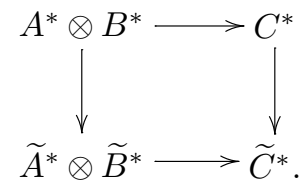

$4^{\mathrm{e}}$ SÉRIE - TOME $35-2002-\mathrm{N}^{\circ} 6$ 
Then the following diagram of cohomology groups commutes

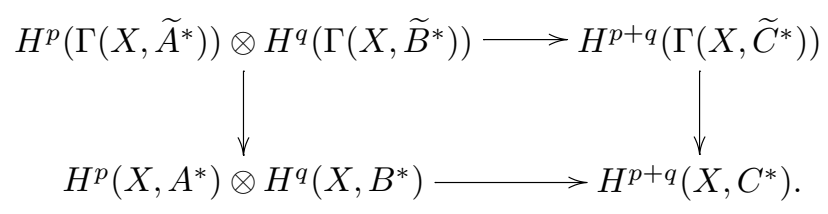

\section{Appendix B. Prespectra and $\Omega$-prespectra}

We recall the naive category of prespectra, which we shall call the category of prespectra, which requires strictly commutative diagrams. For us, a prespectrum is a sequence of pointed spaces $X=\left(X^{0}, X^{1}, \ldots\right)$ together with continuous pointed maps $\phi^{k}: \Sigma X^{k} \rightarrow X^{k+1}$ (or equivalently $\left.\psi^{k}: X^{k} \rightarrow \Omega X^{k+1}\right)$. To simplify matters we always assume that all distinguished points $* \in X^{k}$ are non-degenerate. A morphism of prespectra is a sequence of continuous pointed maps $f^{k}: X^{k} \rightarrow Y^{k}$ such that the following diagrams commute

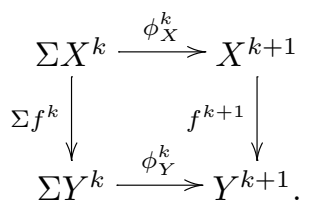

Equivalently one can require the commutativity of the diagrams

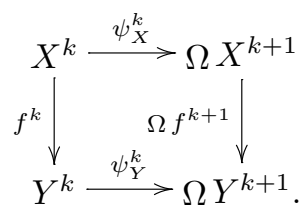

A prespectrum $\left(X^{0}, X^{1}, \ldots\right)$ is called an $\Omega$-prespectrum provided that all maps $\psi^{k}: X^{k} \rightarrow \Omega X^{k+1}$ are homotopy equivalences (of pointed spaces). For each prespectrum $X=\left(X^{0}, X^{1}, \ldots\right)$ we have canonical maps $\pi_{i}\left(X^{k}\right) \rightarrow \pi_{i}\left(\Omega X^{k+1}\right)=\pi_{i+1}\left(X^{k+1}\right)$. The homotopy groups of the prespectrum $X=\left(X^{0}, X^{1}, \ldots\right)$ are defined via the formula

$$
\pi_{n}(X)=\lim _{k \geqslant-n} \pi_{n+k}\left(X^{k}\right) \quad(-\infty<n<\infty) .
$$

Note that if $X$ is an $\Omega$-prespectrum, then $\pi_{n}(X)=\pi_{n+k}\left(X^{k}\right)$ whenever $k \geqslant-n$. A morphism of prespectra $f: X \rightarrow Y$ is called a weak equivalence provided that it induces isomorphisms on all homotopy groups. We will say that the prespectrum $X=\left(X^{0}, X^{1}, \ldots\right)$ is $N$-connected provided that each space $X^{k}$ is $(N+k)$-connected. In the case of $\Omega$-prespectra, this requirement is equivalent to the vanishing of $\pi_{i}(X)$ for all $i \leqslant N$.

Let $f: X \rightarrow Y$ be a morphism of prespectra. Denoting the homotopy fiber (respectively cofiber) of $f^{n}: X^{n} \rightarrow Y^{n}$ by $\mathcal{F}^{n}$ (respectively $\mathcal{C}^{n}$ ) one checks immediately that the sequences $\left(\mathcal{F}^{0}, \mathcal{F}^{1}, \ldots\right)$ and $\left(\mathcal{C}^{0}, \mathcal{C}^{1}, \ldots\right)$ have natural structures of a prespectra, which we denote $f i b(f)$ (respectively cofib $(f)$ ). Moreover $f i b(f)$ is an $\Omega$-prespectrum provided that $X$ and $Y$ are $\Omega$-prespectra. 
Applying the previous remarks to the canonical morphism $X \rightarrow *$ we get for any prespectrum $X$ two new prespectra $\Omega X=\left(\Omega X^{0}, \Omega X^{1}, \ldots\right), \Sigma X=\left(\Sigma X_{0}, \Sigma X_{1}, \ldots\right)$. Moreover the prespectrum $\Omega X$ is an $\Omega$-prespectrum in case $X$ is an $\Omega$-prespectrum, and its homotopy groups are related to that of $X$ via an obvious formula $\pi_{n}(\Omega X)=\pi_{n+1}(X)$. Note finally that for any $X$ we have obvious suspension homomorphisms $\Sigma: \pi_{n}(X) \rightarrow \pi_{n+1}(\Sigma X)$. The Freudenthal Suspension Theorem implies that these maps are isomorphisms (in all degrees) provided that $X$ is $N$-connected for some $N$.

Let $X_{\bullet}$ be a simplicial prespectrum (i.e., a simplicial object in the category of prespectra). Denote the spaces constituting the prespectrum $X_{i}$ by $X_{i}^{n}$. For every $n$ we get a simplicial space $X_{\bullet}^{n}$ and hence may consider its geometric realization $\left|X_{\bullet}^{n}\right|$ or its reduced Segal realization $\left\|X_{\bullet}^{n}\right\|_{\text {red }}=\left\|X_{\bullet}^{n}\right\| /\|*\|$. Moreover for each $n$ we have a canonical pointed map

$$
\Sigma\left(\left\|X_{\bullet}^{n}\right\|_{\text {red }}\right)=\left\|\Sigma X_{\bullet}^{n}\right\|_{\text {red }} \rightarrow\left\|X_{\bullet}^{n+1}\right\|_{\text {red }} .
$$

Thus $\left\|X_{\bullet}\right\|_{r e d}=\left(\left\|X_{\bullet}^{0}\right\|_{r e d},\left\|X_{\bullet}^{1}\right\|_{r e d}, \ldots\right)$ is a prespectrum. In the same way $\left|X_{\bullet}\right|=\left(\left|X_{\bullet}^{0}\right|,\left|X_{\bullet}^{1}\right|\right.$, ...) is also a prespectrum.

The following well-known result (cf. [7, B.7] is of considerable importance for our purposes. We implicitly use the fact that for any simplicial space $X$ • the natural maps

$$
\left\|X_{\bullet}\right\| \leftarrow\left\|n \mapsto\left|\operatorname{Sing}_{\bullet}\left(X_{n}\right)\right|\right\| \rightarrow\left|n \mapsto \operatorname{Sing}_{\bullet}\left(X_{n}\right)\right|
$$

are weak equivalences, where $\left\{n \mapsto \operatorname{Sing} \bullet\left(X_{n}\right)\right\}$ is the the bisimplicial set obtained by applying the singular functor to each $X_{n}$.

PROPOSITION B.1. - Let $X_{\bullet}$ be a simplicial object in the category of $\Omega$-prespectra. Then the prespectrum $\left\|X_{\bullet}\right\|_{\text {red }}$ is an $\Omega$-prespectrum. Moreover if all $\Omega$-prespectra $X_{i}$ are $N$-connected for an appropriate integer $N$, then $\left\|X_{\bullet}\right\|_{r e d}$ is also $N$-connected and there exists a spectral sequence

$$
E_{p, q}^{2}=\pi_{p}\left(\pi_{q}\left(X_{\bullet}\right)\right) \Longrightarrow \pi_{p+q}\left(\left\|X_{\bullet}\right\|_{r e d}\right) .
$$

Combining Proposition B.1 with the theorem of Segal (Proposition 2.2 above) we immediately get the following corollary.

Corollary B.1.1. - Assume in addition that all pointed simplicial spaces $X_{\bullet}^{i}$ are good. Then conclusions of Proposition B.1 apply equally to the prespectrum $\left|X_{\bullet}\right|$.

The naturality of the construction associating the classifying space $B_{\bullet}(A)$ (a simplicial abelian group) to an abelian group $A$ leads to the $\Omega$-prespectrum associated to any simplicial abelian group $A_{\bullet}$ :

$$
\mathbb{B}\left(A_{\bullet}\right)=\left(\left|A_{\bullet}\right|,\left|B_{\bullet}\left(A_{\bullet}\right)\right|,\left|B_{\bullet} B_{\bullet}\left(A_{\bullet}\right)\right|, \ldots\right) .
$$

Here, the $n$th term of the spectrum $\mathbb{B}\left(A_{\bullet}\right)$ is the geometric realization of the resulting $(n+1)$-fold simplicial abelian group.

To any simplicial abelian group $A$. we may associate naturally three complexes - the complex $A_{*}$ with terms $A_{i}$ and differential equal to the alternating sum of the face operations, its Moore complex $\mathcal{M}_{*}\left(A_{\bullet}\right)$ with terms $\mathcal{M}_{i}=\operatorname{Ker} \partial_{1} \cap \cdots \cap \operatorname{Ker} \partial_{i}$ and differential equal to $\partial_{0}$ and finally its normalized complex $K_{N, *}\left(A_{\bullet}\right)$ which is obtained from $A_{*}$ by factoring out the degenerate part. Moreover we have obvious homomorphisms of complexes

$$
\mathcal{M}_{*}\left(A_{\bullet}\right) \rightarrow A_{*} \rightarrow K_{N, *}\left(A_{\bullet}\right)
$$


both of which are homotopy equivalences and whose composition is an isomorphism (so that the complexes $\mathcal{M}_{*}\left(A_{\bullet}\right)$ and $K_{N, *}\left(A_{\bullet}\right)$ may be canonically identified) - see [19,20]. Furthermore we have natural identifications $\pi_{i}\left(A_{\bullet}\right)=H_{i}\left(A_{*}\right)=H_{i}\left(\mathcal{M}\left(A_{\bullet}\right)\right)$. Let $A_{\bullet}$ and $B_{\bullet}$ be two simplicial abelian groups. The Eilenberg-Zilber Theorem shows that the complex $\left(A_{\bullet} \otimes B_{\bullet}\right)_{*}$ is naturally homotopy equivalent to $A_{*} \otimes B_{*}$. The corresponding homotopy equivalence

$$
A_{*} \otimes B_{*} \stackrel{E Z}{\longrightarrow}\left(A_{\bullet} \otimes B_{\bullet}\right)_{*}
$$

is given by the so called shuffle map. Recall that the strictly increasing maps

$$
[i+j] \stackrel{\phi=\left(\phi^{\prime}, \phi^{\prime \prime}\right)}{\longrightarrow}[i] \times[j]
$$

are in one to one correspondence with $(i, j)$-shuffles - see [20]. For any such map $\phi$ we denote by $\varepsilon(\phi)$ the sign of the corresponding shuffle. With these notations the explicit formula for the Eilenberg-Zilber map looks as follows (cf. [19], Chapter 8, §8):

$$
E Z\left(a_{i} \otimes b_{j}\right)=\sum_{\phi=\left(\phi^{\prime}, \phi^{\prime \prime}\right):[i+j] \rightarrow[i] \times[j]} \varepsilon(\phi)\left(\phi^{\prime}\right)^{*}\left(a_{i}\right) \otimes\left(\phi^{\prime \prime}\right)^{*}\left(b_{j}\right) .
$$

Moreover the homomorphism $E Z$ also defines homotopy equivalences on Moore subcomplexes and on normalized quotient complexes.

For any simplicial abelian groups $A_{\bullet}, B_{\bullet}$ we get a canonical map of simplicial sets

$$
A_{\bullet} \wedge B_{\bullet} \rightarrow A_{\bullet} \otimes B_{\bullet}
$$

which defines a pairing in homotopy groups

$$
\pi_{i}\left(A_{\bullet}\right) \times \pi_{j}\left(B_{\bullet}\right) \rightarrow \pi_{i+j}\left(A_{\bullet} \wedge B_{\bullet}\right) \rightarrow \pi_{i+j}\left(A_{\bullet} \otimes B_{\bullet}\right)
$$

For any $x \in \pi_{i}\left(A_{\bullet}\right), y \in \pi_{j}\left(B_{\bullet}\right)$ we denote by $x \otimes y \in \pi_{i+j}\left(A_{\bullet} \otimes B_{\bullet}\right)$ the image of $x \times y$ under this pairing.

The following proposition is well known.

PROPOSITION B.2. - The following diagram commutes

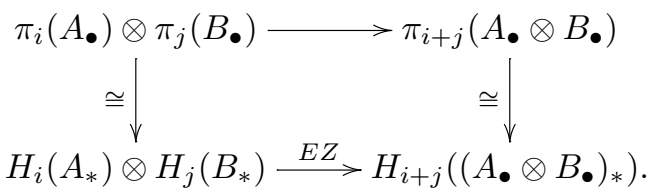

For any simplicial abelian group $A_{\bullet}$, the bisimplicial abelian group $B A_{\bullet}$ may be canonically identified with the tensor product $A_{\bullet} \otimes B_{\bullet} \mathbb{Z}$. Furthermore the complex $(B \bullet \mathbb{Z})_{*}$ is canonically homotopy equivalent to $\mathcal{M}_{*}(B \bullet \mathbb{Z})=\mathbb{Z}[1]$. The Eilenberg-Zilber Theorem now shows that the complex $\left(B A_{\bullet}\right)_{*}$ is canonically homotopy equivalent to the complex $A_{*}[1]$. Using finally the explicit form of the Eilenberg-Zilber map we easily obtain the following result.

LemmA B.3. - For any simplicial abelian group $A_{\text {. }}$. There is a natural homotopy equivalence $A_{*}[1] \stackrel{s}{\rightarrow}\left(B A_{\bullet}\right)_{*}$ given by the formula

$$
a \in A_{n-1} \mapsto\left(s_{0}(a),-s_{1}(a), \ldots,(-1)^{n-1} s_{n-1}(a)\right) \in\left(B A_{n}\right)_{n} .
$$


Assume that $A_{\bullet}^{\prime}, A_{\bullet}^{\prime \prime}, A_{\bullet}$ are simplicial abelian groups and $A_{\bullet}^{\prime} \times A_{\bullet}^{\prime \prime} \stackrel{\phi}{\rightarrow} A_{\bullet}$ is a bilinear map of simplicial sets. The map $\phi$ defines in an obvious way a homomorphism of simplicial abelian groups $A_{\bullet}^{\prime} \otimes A_{\bullet}^{\prime \prime} \rightarrow A_{\bullet}$ and hence a pairing in homotopy groups $\pi_{i}\left(A_{\bullet}^{\prime}\right) \times \pi_{j}\left(A_{\bullet}^{\prime \prime}\right) \rightarrow \pi_{i+j}\left(A_{\bullet}\right)$.

The map $\phi$ defines further two new bilinear maps

$$
\begin{array}{ll}
B A_{\bullet}^{\prime} \times A_{\bullet}^{\prime \prime} \rightarrow B A_{\bullet} & \left(a_{1}^{\prime}, \ldots, a_{p}^{\prime}\right) \times a^{\prime \prime} \mapsto\left(\phi\left(a_{1}^{\prime}, a^{\prime \prime}\right), \ldots, \phi\left(a_{p}^{\prime}, a^{\prime \prime}\right)\right), \\
A_{\bullet}^{\prime} \times B A_{\bullet}^{\prime \prime} \rightarrow B A_{\bullet} & a^{\prime} \times\left(a_{1}^{\prime \prime}, \ldots, a_{p}^{\prime \prime}\right) \mapsto\left(\phi\left(a^{\prime}, a_{1}^{\prime \prime}\right), \ldots, \phi\left(a^{\prime}, a_{p}^{\prime \prime}\right)\right),
\end{array}
$$

and hence two new pairings in homotopy groups

$$
\begin{aligned}
& \pi_{i}\left(A_{\bullet}^{\prime}\right) \times \pi_{j}\left(A_{\bullet}^{\prime \prime}\right)=\pi_{i+1}\left(B A_{\bullet}^{\prime}\right) \times \pi_{j}\left(A_{\bullet}^{\prime \prime}\right) \rightarrow \pi_{i+j+1}\left(B A_{\bullet}\right)=\pi_{i+j}\left(A_{\bullet}\right), \\
& \pi_{i}\left(A_{\bullet}^{\prime}\right) \times \pi_{j}\left(A_{\bullet}^{\prime \prime}\right)=\pi_{i}\left(A_{\bullet}^{\prime}\right) \times \pi_{j+1}\left(B A_{\bullet}^{\prime \prime}\right) \rightarrow \pi_{i+j+1}\left(B A_{\bullet}\right)=\pi_{i+j}\left(A_{\bullet}\right) .
\end{aligned}
$$

LEMMA B.4. - Let $\phi: A_{\bullet}^{\prime} \times A_{\bullet}^{\prime \prime} \rightarrow A_{\bullet}$ be a bilinear map of simplicial abelian groups. Then the following diagrams of complexes commute

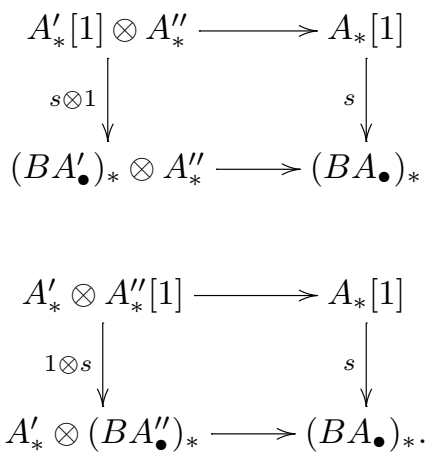

Hence the pairings in homotopy groups defined by the pairings of bisimplicial abelian groups $B A_{\bullet}^{\prime} \times A_{\bullet}^{\prime \prime} \rightarrow B A_{\bullet}, A_{\bullet}^{\prime} \times B A_{\bullet}^{\prime \prime} \rightarrow B A_{\bullet}$ coincide (up to a sign) with pairings in homotopy groups defined by the original pairing $\phi$.

Proof. - The first statement may be verified directly, or deduced from the strict coassociativity of the shuffle map. The second follows immediately from the first.

\section{Appendix C. Functorial formulation of $K$-theory}

We briefly review constructions of Waldhausen, Grayson, and Thomason which facilitate strict functoriality of $K$-theory spectra. We then make explicit our formulation of the definitions of multirelative $K$-theory and the $K$-theory of cosimplicial schemes.

\section{C.1. Waldhausen's $S$-construction}

Let $\mathcal{C}$ be a category with cofibrations and weak equivalences in the sense of Waldhausen [37]. To each such category Waldhausen associates an $\Omega$-prespectrum $\mathcal{K}(\mathcal{C})$ whose definition we recall briefly. We shall assume for simplicity that the category $\mathcal{C}$ has a unique zero object 0 . First of all, Waldhausen defines the category $S_{n}(\mathcal{C})$ of $n$-filtered objects. The objects of this category are chains of cofibrations

$$
0=X_{0} \longmapsto X_{1} \longmapsto \cdots \longmapsto X_{n}
$$


together with the choice of subfactors $X_{i, j}=X_{j} / X_{i}$ for all $i<j$. A morphism in $S_{n}(\mathcal{C})$ from a filtered object $X$ to a filtered object $Y$ is a morphism in $\mathcal{C}$ from $X_{n}$ to $Y_{n}$ which preserves filtrations. A morphism $f: X \rightarrow Y$ is a cofibration (respectively equivalence) in $S_{n}(\mathcal{C})$ provided that the induced morphisms on all subfactors $X_{i, j} \rightarrow Y_{i, j}$ are cofibrations (respectively equivalences) and for that it suffices that the morphisms $X_{i, i+1} \rightarrow Y_{i, i+1}$ be cofibrations (equivalences) for all $i$. One checks immediately that $S_{\bullet}(\mathcal{C})$ is a simplicial category with cofibrations and equivalences. Repeating this construction one gets a bisimplicial category with cofibrations and weak equivalences $S_{\bullet} S_{\bullet}(\mathcal{C})$, etc. Denoting the category of weak equivalences in $S_{\bullet} \ldots S_{\bullet}(\mathcal{C})$ by $w S_{\bullet} \ldots S_{\bullet}(\mathcal{C})$ and regarding each category as a simplicial set in the usual way we get a sequence of spaces

$$
\mathcal{K}^{0}(\mathcal{C})=|w \mathcal{C}|, \quad \mathcal{K}^{1}(\mathcal{C})=\left|w S_{\bullet}(\mathcal{C})\right|, \quad \mathcal{K}^{2}(\mathcal{C})=\left|w S_{\bullet} S_{\bullet}(\mathcal{C})\right|, \ldots
$$

Since $w S_{1}(\mathcal{C})=w \mathcal{C}$, the "1-skeleton in the $S_{\bullet}$ direction" of $\left|w S_{\bullet}(\mathcal{C})\right|$ is homeomorphic to $\Sigma|w \mathcal{C}|$ and similarly for $w S_{\bullet} \ldots S_{\bullet}(\mathcal{C})$, we obtain canonical (pointed) maps

$$
\Sigma \mathcal{K}^{n}(\mathcal{C}) \rightarrow \mathcal{K}^{n+1}(\mathcal{C})
$$

(i.e., a prespectrum). Moreover Waldhausen shows that the associated maps

$$
\mathcal{K}^{n}(\mathcal{C}) \rightarrow \Omega \mathcal{K}^{n+1}(\mathcal{C})
$$

are homotopy equivalences for $n \geqslant 1$, i.e., $\mathcal{K}(\mathcal{C})$ is an $\Omega$-prespectrum.

As always the group $K_{0}(\mathcal{C})=\pi_{0}(\mathcal{K}(\mathcal{C}))$ may be identified with the abelian group with generators $[X](X \in \mathcal{C})$ which are subject to relations

$$
\begin{aligned}
& {[X]=[Y]+[X / Y] \text { for any cofibration } Y \hookrightarrow X,} \\
& {[X]=[Y] \text { for any weak equivalence } X \stackrel{\sim}{\rightarrow} Y .}
\end{aligned}
$$

We finish this brief discussion with the construction of a canonical morphism of prespectra

$$
\mathcal{K}(\mathcal{C}) \rightarrow \mathbb{B}\left(K_{0}(\mathcal{C})\right)
$$

Associating to a filtered object $0=X_{0} \longmapsto X_{1} \longmapsto \cdots \longmapsto X_{n}$ an element $\left(\left[X_{1} / X_{0}\right], \ldots\right.$, $\left.\left[X_{n} / X_{n-1}\right]\right)$ we get a functor from the category $w S_{n}(\mathcal{C})$ to $\left(K_{0}(\mathcal{C})\right)^{n}($ considered as a trivial category). One checks immediately that this construction commutes with face and degeneracy operators and thus provides us with a canonical map of bisimplicial sets

$$
w S_{\bullet}(\mathcal{C}) \rightarrow B_{\bullet}\left(K_{0}(\mathcal{C})\right)
$$

where $B_{\bullet}\left(K_{0}(\mathcal{C})\right)$ is considered as a bisimplicial set trivial in the $q$-direction and hence gives us a canonical pointed continuous map $\left|w S_{\bullet}(\mathcal{C})\right| \rightarrow\left|B_{\bullet}\left(K_{0}(\mathcal{C})\right)\right|$. In the same way we get canonical morphisms

$$
|w \underbrace{S_{\bullet} \ldots S_{\bullet}}_{n}(\mathcal{C})| \rightarrow|\underbrace{B \ldots \ldots B \bullet}_{n}\left(K_{0}(\mathcal{C})\right)|
$$

for all $n \geqslant 0$. 


\section{C.2. Products in $K$-theory}

Let $\mathcal{C}, \mathcal{C}^{\prime}, \mathcal{C}^{\prime \prime}$ be categories with cofibrations and weak equivalences. Assume further that we are given a bifunctor $\otimes: \mathcal{C}^{\prime} \times \mathcal{C}^{\prime \prime} \rightarrow \mathcal{C}$ which is exact in each variable with other variable fixed (i.e., takes 0 to 0 , takes cofibrations to cofibrations and preserves the pushout diagrams involving cofibrations) and also takes weak equivalences to weak equivalences. These properties show that starting with an $i$-filtered object $X \in \mathcal{C}^{\prime}$ and an $j$-filtered object $Y \in \mathcal{C}^{\prime \prime}$ we get an $(i, j)$-bifiltered object $X \otimes Y \in \mathcal{C}$, i.e., $\otimes$ defines a functor from the bisimplicial category $S_{\bullet}\left(\mathcal{C}^{\prime}\right) \times S_{\bullet}\left(\mathcal{C}^{\prime \prime}\right)$ to the bisimplicial category $S_{\bullet} S_{\bullet}\left(\mathcal{C}^{\prime \prime}\right)$. This functor takes weak equivalences to weak equivalences and hence defines a map

$$
\left|w_{\bullet}(\mathcal{C})\right| \wedge\left|w S_{\bullet}(\mathcal{C})\right| \rightarrow\left|w S_{\bullet} S_{\bullet}(\mathcal{C})\right|
$$

These maps define product pairings on homotopy groups $K_{n}\left(\mathcal{C}^{\prime}\right) \times K_{m}\left(\mathcal{C}^{\prime \prime}\right) \rightarrow K_{n+m}(\mathcal{C})$. In the same way one constructs canonical maps of simplicial sets

$$
\mathcal{K}^{n}\left(\mathcal{C}^{\prime}\right) \wedge \mathcal{K}^{m}\left(\mathcal{C}^{\prime \prime}\right) \rightarrow \mathcal{K}^{n+m}(\mathcal{C})
$$

which fit (for all $m, n>0$ ) into commutative diagrams

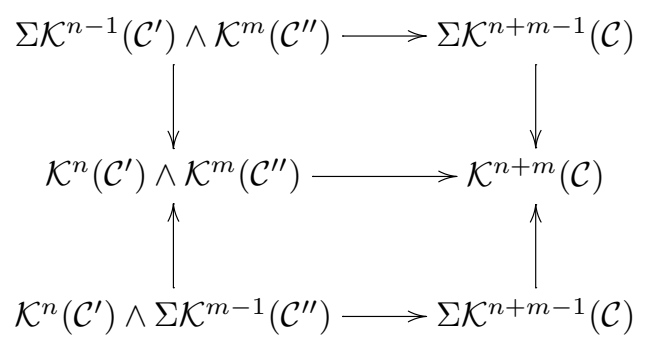

and hence induce the same (as above) pairings in homotopy groups

$$
\begin{aligned}
K_{i}\left(\mathcal{C}^{\prime}\right) \times K_{j}\left(\mathcal{C}^{\prime \prime}\right) & =\pi_{i+n}\left(\mathcal{K}^{n}\left(\mathcal{C}^{\prime}\right)\right) \times \pi_{j+m}\left(\mathcal{K}^{m}\left(\mathcal{C}^{\prime \prime}\right)\right) \rightarrow \pi_{i+j+n+m}\left(\mathcal{K}^{n+m}(\mathcal{C})\right) \\
& =K_{i+j}(\mathcal{C}) .
\end{aligned}
$$

\section{C.3. $K$-theory with finite coefficients}

Fix an integer $l \geqslant 2$ and denote by $M_{l}^{2}$ the mod-l Moore space defined as the cone of multiplication by $l$ endomorphism of $S^{1}$. The homotopy groups with mod-l coefficients of a pointed space $X$ are defined via the formula

$$
\pi_{i}(X, \mathbb{Z} / l)=\left[S^{i-2} \wedge M_{l}^{2}, X\right] \text { if } i \geqslant 2 .
$$

and of a prespectrum $X=\left(X^{0}, X^{1}, \ldots\right)$ by

$$
\pi_{i}(X, \mathbb{Z} / l)=\underset{k \geqslant-i+2}{\lim _{\overrightarrow{2}}} \pi_{i+k}\left(X^{k}, \mathbb{Z} / l\right) \quad(-\infty<i<\infty)
$$

For a category $\mathcal{C}$ with cofibrations and weak equivalences we define the $K$-groups of $\mathcal{C}$ with finite coefficients as homotopy groups with finite coefficients of the corresponding prespectrum:

$$
K_{i}(\mathcal{C}, \mathbb{Z} / l)=\pi_{i}\left(\mathcal{K}^{*}(\mathcal{C}), \mathbb{Z} / l\right)
$$


Returning to the situation discussed in Section C.2, we note first that the pairing of spaces $\mathcal{K}^{n}\left(\mathcal{C}^{\prime}\right) \wedge \mathcal{K}^{m}\left(\mathcal{C}^{\prime \prime}\right) \rightarrow \mathcal{K}^{n+m}(\mathcal{C})$ defines pairings in homotopy groups with coefficients (see discussion after Proposition 14.2, where we take $M^{\prime}=S^{0}, M^{\prime \prime}=M_{l}^{2}, M=M_{l}^{2}$ )

$$
\begin{aligned}
& \pi_{i}\left(\mathcal{K}^{n}\left(\mathcal{C}^{\prime}\right)\right) \times \pi_{j}\left(\mathcal{K}^{m}\left(\mathcal{C}^{\prime \prime}\right), \mathbb{Z} / l\right) \rightarrow \pi_{i+j}\left(\mathcal{K}^{n+m}(\mathcal{C}), \mathbb{Z} / l\right) \quad i \geqslant 0, j \geqslant 2, \\
& \pi_{i}\left(\mathcal{K}^{n}\left(\mathcal{C}^{\prime}\right), \mathbb{Z} / l\right) \times \pi_{j}\left(\mathcal{K}^{m}\left(\mathcal{C}^{\prime \prime}\right)\right) \rightarrow \pi_{i+j}\left(\mathcal{K}^{n+m}(\mathcal{C}), \mathbb{Z} / l\right) \quad i \geqslant 2, j \geqslant 0 .
\end{aligned}
$$

These pairings define pairings in $K$-groups with coefficients

$$
\begin{aligned}
& K_{i}\left(\mathcal{C}^{\prime}\right) \times K_{j}\left(\mathcal{C}^{\prime \prime}, \mathbb{Z} / l\right) \rightarrow K_{i+j}(\mathcal{C}, \mathbb{Z} / l), \\
& K_{i}\left(\mathcal{C}^{\prime}, \mathbb{Z} / l\right) \times K_{j}\left(\mathcal{C}^{\prime \prime}\right) \rightarrow K_{i+j}(\mathcal{C}, \mathbb{Z} / l),
\end{aligned}
$$

which are independent of the choice of $n, m \geqslant 2$.

To define pairings on the $K$-groups with coefficients, we need to fix a comultiplication $\Sigma^{2} M_{l}^{2} \stackrel{c}{\rightarrow} M_{l}^{2} \wedge M_{l}^{2}$ as in Proposition 16.1, which is required to be homotopy right inverse to the canonical map $M_{l}^{2} \wedge M_{l}^{2} \rightarrow S^{2} \wedge M_{l}^{2}=\Sigma^{2} M_{l}^{2}$. It is known that such a map exists in case $l \not \equiv 2 \bmod 4$ and is uniquely (up to homotopy) defined by the above requirement in case $l$ is odd - see [8]. Thus in case $l \not \equiv 2 \bmod 4$ we may define pairings in $K$-groups with finite coefficients

$$
K_{i}\left(\mathcal{C}^{\prime}, \mathbb{Z} / l\right) \times K_{j}\left(\mathcal{C}^{\prime \prime}, \mathbb{Z} / l\right) \rightarrow K_{i+j}(\mathcal{C}, \mathbb{Z} / l) .
$$

However these pairings in general depend on the choice of the map $c$. Finally we get really well-defined product maps on $K$-groups with coefficients $\mathbb{Z} / l$ for all odd $l$ 's.

In the main body of the paper we need a few facts concerning products in homotopy groups with finite coefficients in the case of simplicial abelian groups. We start with the following elementary observation.

LEMma C.3.1. - For any simplicial abelian group A• we have natural isomorphisms

$$
\pi_{i}\left(\left|A_{\bullet}\right|, \mathbb{Z} / l\right)=H_{i}\left(C_{l}\left(A_{*}\right)\right),
$$

where $C_{l}\left(A_{*}\right)=C\left(A_{*} \stackrel{l}{\rightarrow} A_{*}\right)$ is the cone of the multiplication by l homomorphism.

The correspondence $A_{*} \mapsto C_{l}\left(A_{*}\right)$ defines a functor from the category of complexes to itself. Moreover for any complexes $A_{*}, B_{*}$ we have a natural homomorphism

$$
C_{l}\left(A_{*}\right) \otimes C_{l}\left(B_{*}\right) \rightarrow C_{l}\left(A_{*} \otimes B_{*}\right) .
$$

Thus for any simplicial abelian groups $A_{\bullet}$ and $B \bullet$ we get a natural homomorphism of complexes

$$
C_{l}\left(A_{*}\right) \otimes C_{l}\left(B_{*}\right) \rightarrow C_{l}\left(A_{*} \otimes B_{*}\right) \stackrel{C_{l}(E Z)}{\longrightarrow} C_{l}\left(\left(A_{\bullet} \otimes B_{\bullet}\right)_{*}\right) .
$$

LEMMA C.3.2. - For any simplicial abelian groups $A_{\bullet}, B_{\bullet}$ we have a commutative diagram

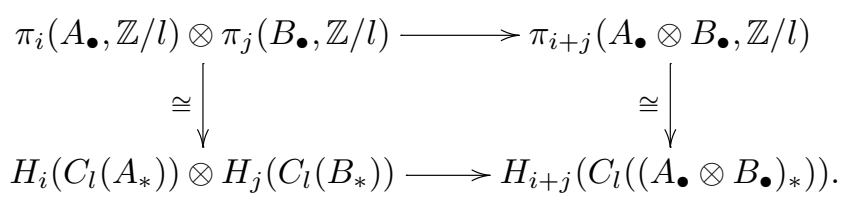




\section{C.4. Big vector bundles}

Let $X$ be a Noetherian scheme. Consider the big Zariski site $S c h / X$ of all schemes of finite type over $X$. Let $P: S c h / X \rightarrow A b$ be an $\mathcal{O}$-module. For every $Y \in S c h / X$ restricting $P$ to the small Zariski site of $Y$ gives an $\mathcal{O}_{Y}$-module $P_{Y}$. Following Grayson [12], we say that $P$ is a big vector bundle on $X$ provided that the following conditions hold

(1) For every $Y \in S c h / X$ the $\mathcal{O}_{Y}$-module $P_{Y}$ is a vector bundle on $Y$ (i.e., a locally free, coherent $\mathcal{O}_{Y}$-module).

(2) For every arrow $f: Y \rightarrow Z$ in $S c h / X$ the induced homomorphism $f^{*}\left(P_{Z}\right) \rightarrow P_{Y}$ is an isomorphism (obviously it suffices to require that the above condition holds for the structure morphisms $Y \rightarrow X$ only).

In other words a big vector bundle on $X$ is a family of vector bundles $P_{Y}(Y \in S c h / X)$ together with a data of isomorphisms $f^{*}\left(P_{Z}\right) \rightarrow P_{Y}$ which satisfy appropriate compatibility conditions. Denote by $\mathcal{P}(S c h / X)$ the category of big vector bundles considered as a full subcategory in the category of $\mathcal{O}$-modules. Denoting the category of vector bundles on $X$ by $\mathcal{P}(X)$, we have an obvious functor $\mathcal{P}(S c h / X) \rightarrow \mathcal{P}(X)$. One checks easily (cf. [12]) that this functor is an equivalence. For every scheme of finite type $f: Y \rightarrow X$ we have an obvious restriction functor $\mathcal{P}(S c h / X) \rightarrow \mathcal{P}(S c h / Y)$ which we denote $f^{*}$. The main advantage of this construction is that whenever we have a chain of arrows $Z \stackrel{g}{\rightarrow} Y \stackrel{f}{\rightarrow} X$ the functors $(f \circ g)^{*}$ and $g^{*} \circ f^{*}$ coincide (not simply agree up to a canonical isomorphism).

\section{C.5. The $\Omega$-prespectra of algebraic $K$-theory}

Let $X$ be a Noetherian scheme. The category $\mathcal{P}(X)$ is an exact category in the sense of Quillen [25] and hence one can apply Quillen's Q-construction [25] or Waldhausen's S-construction [37] to get the corresponding $K$-theory prespectrum. However it will be more convenient for our purposes to follow the approach developed by Thomason in [32] (replacing also the category $\mathcal{P}(X)$ by the equivalent category $\mathcal{P}(S c h / X))$.

Denote by $\mathcal{C P}(X)$ the category of bounded complexes of big vector bundles on $X$. The category $\mathcal{C P}(X)$ may be considered as a Waldhausen category, in which cofibrations are degreewise split monomorphisms and equivalences are quasi-isomorphisms. We denote by $\mathcal{K}(X)$ the $\Omega$-prespectrum corresponding to the category $\mathcal{C P}(X)$. It is known [32] that this $\Omega$-prespectrum is equivalent to the usual $\Omega$-prespectrum of algebraic $K$-theory. Let $f: Y \rightarrow X$ be a scheme of finite type over $X$. The exact functor $f^{*}: \mathcal{C P}(S c h / X) \rightarrow \mathcal{C P}(S c h / Y)$ defines a morphism of prespectra $f^{*}: \mathcal{K}(X) \rightarrow \mathcal{K}(Y)$. Furthermore if $g: Z \rightarrow Y$ is a scheme of finite type over $Y$ then the diagram of prespectra

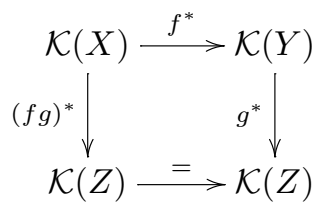

strictly commutes.

Assume now that we are given a family of closed subschemes $\left\{X_{i} \subset X\right\}_{i \in I}$. Denote by $\mathcal{C P} \mathcal{P}^{\left\{X_{i}\right\}}(X)$ the full subcategory of $\mathcal{C P}(X)$ consisting of complexes acyclic outside of $\bigcup_{i \in I} X_{i}$. The category $\mathcal{C} \mathcal{P}^{\left\{X_{i}\right\}}(X)$ is a Waldhausen category in its own right. We denote by $\mathcal{K}^{\left\{X_{i}\right\}}(X)$ the corresponding $\Omega$-prespectrum (this is called the prespectrum of $K$-theory with supports in $\left.\left\{X_{i}\right\}_{i \in I}\right)$. The first term of this $\Omega$-prespectrum is the space $\left|w S_{\bullet}\left(\mathcal{C P}^{\left\{X_{i}\right\}}(X)\right)\right|$, so that

$$
\Omega^{-1} \mathcal{K}^{\left\{X_{i}\right\}}(X) \cong\left|w S_{\bullet}\left(\mathcal{C} \mathcal{P}^{\left\{X_{i}\right\}}(X)\right)\right|
$$


We usually use the abbreviated notation $\left|S_{\bullet}^{\left\{X_{i}\right\}}(X)\right|$ for this space. Assume further that $f: Y \rightarrow X$ is a scheme of finite type over $X$ and $\left\{Y_{j}\right\}_{j \in J}$ is a family of closed subschemes of $Y$. The exact functor $f^{*}: \mathcal{C P}(X) \rightarrow \mathcal{C P}(Y)$ takes $\mathcal{C} \mathcal{P}^{\left\{X_{i}\right\}}(X)$ to $\mathcal{C} \mathcal{P}^{\left\{Y_{j}\right\}}(Y)$ provided that $f^{-1}\left(X_{i}\right) \subset \bigcup_{j \in J} Y_{j}$ for all $i \in I$. In this case we get the induced morphism of prespectra $f^{*}: \mathcal{K}^{\left\{X_{i}\right\}}(X) \rightarrow \mathcal{K}^{\left\{Y_{j}\right\}}(Y)$. Once again the formula $(f g)^{*}=g^{*} f^{*}: \mathcal{K}^{\left\{X_{i}\right\}}(X) \rightarrow \mathcal{K}^{\left\{Z_{k}\right\}}(Z)$ holds whenever both sides make sense.

Since the $\Omega$-prespectrum provided by the Waldhausen construction is always $(-1)$-connected we conclude that the $\Omega$-prespectra $\mathcal{K}(X)$ and $\mathcal{K}^{\left\{X_{i}\right\}}(X)$ are $(-1)$-connected.

\section{C.6. Relative and multirelative $K$-theory}

Let $X$ be a Noetherian scheme and $Y \subset X$ a closed subscheme of $X$. The $\Omega$-prespectrum of algebraic $K$-theory of $X$ relative to $Y$ is defined as the homotopy fiber of the morphism of prespectra $\mathcal{K}(X) \rightarrow \mathcal{K}(Y)$ :

$$
\mathcal{K}(X ; Y)=f i b(\mathcal{K}(X) \rightarrow \mathcal{K}(Y))
$$

and the relative $K$-groups are defined as homotopy groups of this prespectrum:

$$
K_{i}(X ; Y)=\pi_{i}(\mathcal{K}(X ; Y)) .
$$

More generally if we are given a family of closed subschemes $Y_{0}, \ldots, Y_{n-1} \subset X$ one defines the $\Omega$-prespectrum of multirelative $K$-theory inductively, setting

$$
\begin{aligned}
& \mathcal{K}\left(X ; Y_{0}, \ldots, Y_{n-1}\right) \\
& \quad=f i b\left(\mathcal{K}\left(X ; Y_{0}, \ldots, Y_{n-2}\right) \rightarrow \mathcal{K}\left(Y_{n-1} ; Y_{0} \cap Y_{n-1}, \ldots, Y_{n-2} \cap Y_{n-1}\right)\right) .
\end{aligned}
$$

One may rephrase the above construction in slightly different terms. The above data defines a $n$-cube in the category of prespectra $\mathcal{K}_{S}=\mathcal{K}\left(\bigcap_{i \notin S} Y_{i}\right)$, which we might denote by $\mathcal{K}_{\bullet}, \ldots, \bullet$ Thus,

$$
\mathcal{K}\left(X ; Y_{0}, \ldots, Y_{n-1}\right) \equiv f i b\left\{\operatorname{cube}_{n}\left(\mathcal{K}_{\bullet}, \ldots, \bullet\right)\right\}
$$

(see Sections 3, 4 for a detailed discussion of this construction). Assume finally that each of the intersection schemes $Y_{i_{0}} \cap \cdots \cap Y_{i_{k}}$ is provided with a family of supports $\mathcal{F}\left(Y_{i_{0}} \cap \cdots \cap Y_{i_{k}}\right)$ such that for any $Z \in \mathcal{F}\left(Y_{i_{0}} \cap \cdots \cap Y_{i_{k}}\right)$ and any $i_{k+1} \neq i_{0}, \ldots, i_{k}$ the intersection of $Z$ with $Y_{i_{k+1}}$ is contained in $\mathcal{F}\left(Y_{i_{0}} \cap \cdots \cap Y_{i_{k}} \cap Y_{k+1}\right)$. In this case we can repeat the previous construction replacing everywhere the prespectra of $K$-theory by prespectra of $K$-theory with supports. We denote the corresponding $\Omega$-prespectrum by $\mathcal{K}^{\mathcal{F}}\left(X ; Y_{0}, \ldots Y_{n-1}\right)$. It follows immediately from the definitions that the $\Omega$-prespectrum $\mathcal{K}^{\mathcal{F}}\left(X ; Y_{0}, \ldots Y_{n-1}\right)$ is $(-n-1)$-connected.

\section{C.7. $K$-theory of cosimplicial schemes}

Let $X^{\bullet}$ be a Noetherian cosimplicial scheme. The $\Omega$-prespectra $\mathcal{K}\left(X^{n}\right)$ form a simplicial $\Omega$-prespectrum and we define the $\Omega$-prespectrum $\mathcal{K}\left(X^{\bullet}\right)$ as the geometric realization of this simplicial $\Omega$-prespectrum. Assume now that each of the schemes $X^{n}$ is provided with a family of supports $\mathcal{F}\left(X^{n}\right)$ in such a way that for any $Y \in \mathcal{F}\left(X^{n}\right)$ and any structure morphism $X^{m} \rightarrow X^{n}$ the inverse image of $Y$ in $X^{m}$ is in $\mathcal{F}\left(X^{m}\right)$. Then we can repeat the previous construction replacing everywhere $\mathcal{K}\left(X^{n}\right)$ by $\mathcal{K}^{\mathcal{F}\left(X^{n}\right)}\left(X^{n}\right)$ thus getting the $\Omega$-prespectrum of $K$-theory with supports in $\mathcal{F}$ :

$$
\mathcal{K}^{\mathcal{F}}\left(X^{\bullet}\right)=\left|n \mapsto \mathcal{K}^{\mathcal{F}\left(X^{n}\right)}\left(X^{n}\right)\right|
$$




\section{Appendix D. Brown-Gersten techniques for simplicial sheaves}

The globalization construction of Brown-Gersten involves the formulation of hypercohomology of a Noetherian scheme $X$ with coefficients in a pointed simplicial sheaf $K$,

$$
H^{q}(X, K)=\pi_{-q} R \Gamma(X, K)
$$

Let $X$ be a Noetherian scheme. Recall (see [9]) that a morphism $p: E \rightarrow B$ of simplicial sheaves on $X$ is said to be a global fibration provided that for any inclusion $U \subset V$ of open sets the map of simplicial sets

$$
E(V) \stackrel{(p(V), \text { res })}{\longrightarrow} B(V) \times_{B(U)} E(U)
$$

is a Kan fibration. Taking here $U=\emptyset$ and noting that $E(\emptyset)=*$ for any simplicial sheaf $E$, we see in particular that $E(V) \stackrel{p(V)}{\longrightarrow} B(V)$ is a Kan fibration for any open $V \subset X$.

A simplicial sheaf $E$ is called flasque (or fibrant) if the natural morphism $E \rightarrow *$ is a global fibration. In other words $E$ is fibrant, provided that for any open subsets $U \subset V \subset X$ the restriction map $E(V) \rightarrow E(U)$ is a Kan fibration. Taking here $U=\emptyset$ we conclude in particular that $E(V)$ is a Kan complex for any open $V \subset X$.

A morphism $p: E \rightarrow B$ of simplicial sheaves is said to be a weak equivalence provided that for each point $x \in X$ the induced map on stalks $p_{x}: E_{x} \rightarrow B_{x}$ is a weak equivalence of simplicial sets.

Finally a morphism $p: E \rightarrow B$ of simplicial sheaves is said to be a cofibration provided that it is injective (the definition given in [9] is different but it is not hard to see that it amounts to the same thing).

A morphism of simplicial sheaves which is both a global fibration (respectively cofibration) and a weak equivalence is called a trivial (global) fibration (respectively a trivial cofibration).

THEOREM D.1 [9]. - The category of simplicial sheaves on $X$ with the above notions of (global) fibration, weak equivalence and cofibration is a closed model category in the sense of Quillen [24]. In particular the following statements hold

(1) (The lifting property). For any commutative diagram of simplicial sheaves

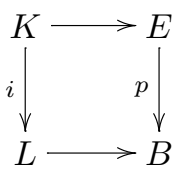

in which $i$ is a cofibration, $p$ is a global fibration and either $i$ or $p$ is a weak equivalence there exists a morphism $s: L \rightarrow E$ for which the resulting diagram still commutes.

(2) (The factorization property). Any morphism $f: K \rightarrow L$ of simplicial sheaves may be factored as $f=p i$, where $i$ is a cofibration, $p$ is a global fibration and either $i$ or $p$ can be taken to be a weak equivalence.

If $A$ is a simplicial set then we keep the same notation $A$ for the corresponding constant simplicial sheaf (i.e., the sheaf associated to the presheaf $U \mapsto A$ ). For a simplicial sheaf $K$ the product sheaf $K \times A$ coincides obviously with the simplicial sheaf associated to the presheaf $U \mapsto K(U) \times A$. We use the notation $\mathrm{I}=\mathrm{I}$. for the standard simplicial interval (i.e., $\mathrm{I}=\Delta^{1}$ ) and also for the corresponding constant simplicial sheaf. We say that two morphisms of simplicial 
sheaves $g_{0}, g_{1}: K \rightarrow L$ are homotopic provided there exists a morphism $H: K \times \mathrm{I} \rightarrow L$ such that $H_{\mid K \times 0}=g_{0}, H_{\mid K \times 1}=g_{1}$. Note that in this case the induced morphisms of simplicial sets $g_{0}(U), g_{1}(U): K(U) \rightarrow L(U)$ are homotopic for every open $U \subset X$.

The following lemma, a standard consequence of Theorem D.1, will be used sufficiently often that we provide a simple proof.

LEMMA D.2. - For any commutative diagram of simplicial sheaves

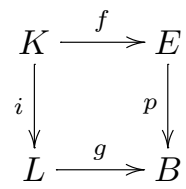

in which $i$ is a cofibration, $p$ is a global fibration and either $i$ or $p$ is a weak equivalence, a lifting $s: L \rightarrow E$ (which makes the total diagram commutative) is defined uniquely up to a fiberwise homotopy constant on $K$ (i.e., a homotopy $L \times I \rightarrow E$ whose composition with $p$ is $g$ composed with the projection $L \times I \rightarrow L$, and whose restriction to $K \times I$ is given by $f$ composed with the projection $K \times I \rightarrow K)$.

Proof. - Let $s_{0}, s_{1}: L \rightarrow E$ be two such liftings. Consider the following diagram

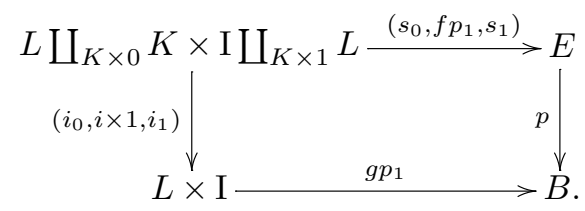

This diagram obviously commutes, the right vertical arrow is still a global fibration and the left vertical arrow is obviously a cofibration. Moreover the left vertical arrow is a trivial cofibration in case $i$ is a trivial cofibration. Thus the lifting property applies and gives us a morphism $H: L \times \mathrm{I} \rightarrow E$ such that $H_{\mid L \times 0}=s_{0}, H_{\mid L \times 1}=s_{1}$.

Applying the factorization property to the canonical morphism $K \rightarrow *$ we conclude that for any simplicial sheaf $K$ there exists a trivial cofibration $i: K \rightarrow J$ from $K$ to a flasque simplicial sheaf $J$. In this situation we say that $J$ is a flasque resolution of $K$. For any open subscheme $U \subset X$ we define the simplicial set $R \Gamma(U, K)$ via the formula $R \Gamma(U, K)=J(U)$ (where $J$ is a flasque resolution of $K$ ). Note that according to the definition of a flasque sheaf the simplicial set $J(U)$ is a Kan complex. One defines the hypercohomology of $X$ with coefficients in the pointed simplicial sheaf $K$ using the formula

$$
H^{q}(X, K)=\pi_{-q} R \Gamma(X, K) .
$$

Note that $H^{0}$ in general is just a pointed set and the group $H^{-1}$ in general need not be abelian.

One important consequence of Lemma D.2 is the following proposition.

Proposition D.3. - Let $i: K \rightarrow J$ be a flasque resolution of $K$ and let $f: K \rightarrow L$ be any morphism from $K$ to a flasque simplicial sheaf $L$. Then $f$ extends to a morphism $g: J \rightarrow L$ and moreover this extension is defined uniquely up to homotopy constant on $K$.

Consequently, the simplicial set $R \Gamma(U, K)$ is defined uniquely up to (a unique up to a homotopy) homotopy equivalence and depends functorially on K. Thus the hypercohomology groups are defined uniquely up to a unique isomorphism and depend functorially on $K$. 
We recall from [9] the following property of a weak equivalence of flasque simplicial sheaves.

LEMMA D.4 [9]. - Let $f: K \rightarrow L$ be a weak equivalence of flasque simplicial sheaves. Then for any open $U \subset X$ the corresponding morphism of simplicial sets $K(U) \stackrel{f(U)}{\longrightarrow} L(U)$ is also a weak equivalence.

Proposition D.5.- Assume we are given a sequence of pointed simplicial sheaves $K^{\prime} \stackrel{f^{\prime}}{\rightarrow} K \stackrel{f}{\rightarrow} K^{\prime \prime}$ such that the composition $f \circ f^{\prime}$ maps $K^{\prime}$ to the distinguished point of $K^{\prime \prime}$ and for any point $x \in X$ the corresponding sequence of stalks at $x$

$$
\left|K_{x}^{\prime}\right| \rightarrow\left|K_{x}\right| \rightarrow\left|K_{x}^{\prime \prime}\right|
$$

is a homotopy fibration sequence. Then there exists a commutative diagram

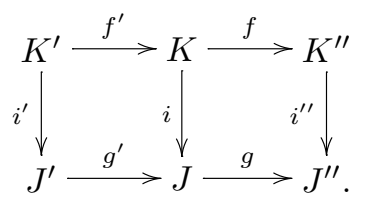

such that the vertical maps are flasque resolutions, $g$ is a global fibration and the composition $g \circ g^{\prime}$ maps $J^{\prime}$ to the distinguished point of $J^{\prime \prime}$. In this case

$$
\left|J^{\prime}(U)\right| \stackrel{g^{\prime}(U)}{\longrightarrow}|J(U)| \stackrel{g(U)}{\longrightarrow}\left|J^{\prime \prime}(U)\right|
$$

is a homotopy fibration sequence for any open $U \subset X$.

Proof. - Choose flasque resolutions $K^{\prime \prime} \stackrel{i^{\prime \prime}}{\rightarrow} J^{\prime \prime}, K^{\prime} \stackrel{i^{\prime}}{\rightarrow} J^{\prime}$. Applying the factorization property to the morphism $K \stackrel{f}{\rightarrow} K^{\prime \prime} \stackrel{i^{\prime \prime}}{\rightarrow} J^{\prime \prime}$ we get a flasque resolution $K \stackrel{i}{\rightarrow} J$ and a global fibration $g: J \rightarrow J^{\prime \prime}$ such that $g i=i^{\prime \prime} f$. Let $\mathcal{F}$ denote the fiber of $g$ over the distinguished point. Applying Proposition D.3 to the morphism $K^{\prime} \rightarrow \mathcal{F}$ we get a morphism $J^{\prime} \stackrel{g^{\prime}}{\rightarrow} J$ such that the composition $g \circ g^{\prime}$ is trivial and the diagram (D.5.1) commutes.

Since $g$ is a global fibration we conclude that $g(U)$ is a Kan fibration and hence $|g(U)|:|J(U)| \rightarrow\left|J^{\prime \prime}(U)\right|$ is a Serre fibration of topological spaces. This implies that the homotopy fiber of $|g(U)|$ is canonically weakly equivalent to the usual fiber, i.e., to $|\mathcal{F}(U)|$. Thus it would suffice to show that the map $\left|J^{\prime}(U)\right| \stackrel{g^{\prime}(U)}{\longrightarrow}|\mathcal{F}(U)|$ is a weak equivalence. However this follows immediately from Lemma D.4.

COROLlary D.5.1. - In conditions and notations of Proposition D.5 assume further that the simplicial sheaves $K^{\prime}, K, K^{\prime \prime}$ are flasque. Then for any open $U \subset X$ the sequence

$$
\left|\Gamma\left(U, K^{\prime}\right)\right| \rightarrow|\Gamma(U, K)| \rightarrow\left|\Gamma\left(U, K^{\prime \prime}\right)\right|
$$

is a homotopy fibration sequence.

Proof. - This follows immediately from the presence of a commutative diagram

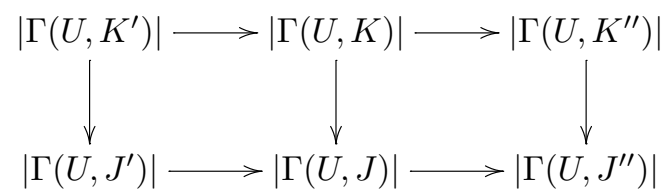


whose vertical arrows are weak equivalences and whose bottom row is a homotopy fibration sequence.

The following important result provides us with a canonical way to resolve simplicial sheaves.

Proposition D.6 [13, 7.65], [21]. - For any Noetherian scheme $X$ of finite Krull dimension there exists a functor $\mathcal{R}: L \mapsto \mathcal{R}(L)$ from the category of simplicial sheaves on $X$ to itself and a natural transformation $I d \stackrel{\phi}{\rightarrow} \mathcal{R}$ with the following properties

(1) The functor $\mathcal{R}$ commutes with finite products and, in particular takes the trivial simplicial sheaf to itself.

(2) Consequently, a pairing $L^{\prime} \wedge L^{\prime \prime} \rightarrow L$ of simplicial sheaves induces a pairing $\mathcal{R}\left(L^{\prime}\right) \wedge \mathcal{R}\left(L^{\prime \prime}\right) \rightarrow \mathcal{R}(L)$.

(3) For any simplicial sheaf $L$, the simplicial sheaf $\mathcal{R}(L)$ is flasque and the morphism $\phi_{L}: L \rightarrow \mathcal{R}(L)$ is a weak equivalence.

Proof. - The functor $\mathcal{R}$ is the composition $\mathcal{R}=G \circ E x$ of the Kan's functor $E x$ with the Godement resolution functor $G$ (cf. discussion at beginning of Appendix A). Applying the functor $E x$ to $L$ we get a simplicial sheaf $E x(L)$, whose stalks are Kan complexes and a weak equivalence $L \rightarrow \operatorname{Ex}(L)$. The functor $G$ takes a stalkwise fibration $E \rightarrow B$ (i.e., for all $x \in X$, the map of simplicial sets $E_{x} \rightarrow B_{x}$ obtained by taking the stalk at $x$ is a Kan fibration) to a global fibration $G(E) \rightarrow G(B)$; namely, for any inclusion $U \subset V$ of open sets, the restriction map

$$
\prod_{v \in V} E_{v} \stackrel{(p(V), \mathrm{res})}{\longrightarrow} \prod_{v \in V} B_{v} \times \prod_{u \in U} B_{u} \prod_{u \in U} E_{u}=\prod_{w \in V \backslash U} B_{w} \times \prod_{u \in U} E_{u}
$$

is given by the natural projection and thus is a Kan fibration. In particular, the functor takes every simplicial sheaf whose stalks are Kan complexes to a fibrant (= flasque) sheaf. Finally the natural morphism $L \rightarrow G(L)$ is a weak equivalence for any $L$ provided that the cohomological dimension of abelian sheaves on $X$ is bounded above - see [21] or [13]. Thus it suffices now to use the theorem of Grothendieck showing that cohomological dimension of abelian sheaves on $X$ is bounded by $\operatorname{dim} X$.

From this point on we assume that $X$ is a Noetherian scheme of finite Krull dimension and we always use the canonical flasque resolution $\mathcal{R}(L)$ when talking about the simplicial set $R \Gamma(U, L)$, i.e., set $R \Gamma(U, L)=\Gamma(U, \mathcal{R}(L))$.

LemmA D.7. - With the conditions and notations of Proposition D.5, the sequence

$$
R \Gamma\left(U, K^{\prime}\right) \rightarrow R \Gamma(U, K) \rightarrow R \Gamma\left(U, K^{\prime \prime}\right)
$$

is a homotopy fibration sequence for all $U$. In particular, we have a long exact homotopy sequence

$$
\begin{aligned}
\pi_{i+1}(R \Gamma(U, K)) \stackrel{f_{*}}{\rightarrow} \pi_{i+1}\left(R \Gamma\left(U, K^{\prime \prime}\right)\right) \stackrel{\partial}{\rightarrow} \pi_{i}\left(R \Gamma\left(U, K^{\prime}\right)\right) \stackrel{f_{*}^{\prime}}{\rightarrow} \pi_{i}(R \Gamma(U, K)) \\
\rightarrow \cdots \rightarrow \pi_{0}(R \Gamma(U, K)) \stackrel{f_{*}}{\rightarrow} \pi_{0}\left(R \Gamma\left(U, K^{\prime \prime}\right)\right)
\end{aligned}
$$


Proof. - This follows immediately from the commutative diagram

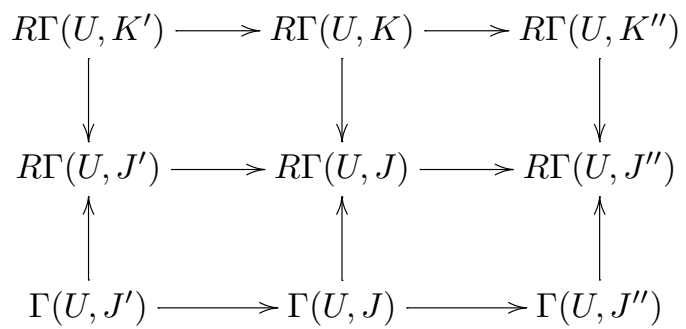

whose vertical arrows are weak equivalences according to Lemma D.4 and whose bottom row is a homotopy fibration sequence according to Proposition D.5.

COROLlARY D.7.1. - Consider a commutative diagram

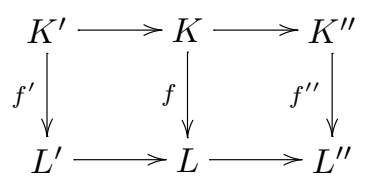

whose rows satisfy the conditions of Proposition D.5. Applying the functor $R \Gamma$ to this diagram, we get a commutative diagram whose rows are homotopy fibration sequences

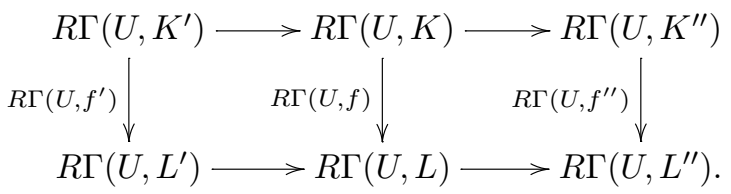

Thus, in particular, for any open $U \subset X$ the following diagram of homotopy groups commutes

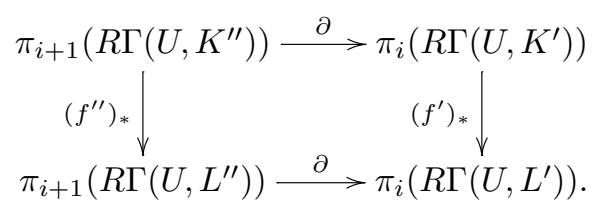

\section{Appendix E. Closed cofibrations}

In this short appendix we gathered some of the facts about closed cofibrations of topological spaces which are used in the main body of the paper.

Recall that a map $i: A \hookrightarrow X$ is called a closed cofibration iff it is a closed embedding and satisfies the homotopy extension property with respect to maps to all spaces or equivalently if $X \times 0 \cup A \times \mathrm{I}$ is a retract of $X \times \mathrm{I}$. The following easy observation (used in Section 2) is a part of a Theorem proved by Puppe [23].

LEMMA E.1. - Every closed cofibration $A \subset X$ is a zero set in $X$, i.e. there exists a continuous function $u: X \rightarrow$ I such that $A=u^{-1}(0)$. 
PROPOSITION E.2. - Consider a commutative diagram of pointed spaces with non-degenerate distinguished points

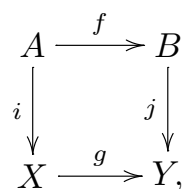

in which $i$ and $j$ are closed cofibrations. Then the induced maps

$$
f i b(f) \rightarrow f i b(g) \quad \text { and } \quad \operatorname{cofib}(f) \rightarrow \operatorname{cofib}(g)
$$

are also closed cofibrations (and distinguished points of $f i b(f), \operatorname{cofib}(f), \ldots$ are non-degenerate).

Proof. - An immediate verification shows that the maps $A^{\mathrm{I}} \rightarrow X^{\mathrm{I}}$ and $B^{\mathrm{I}} \rightarrow Y^{\mathrm{I}}$ are still closed cofibrations - see [28]. Consider next the commutative diagram

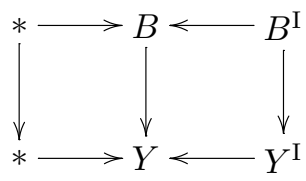

All the vertical arrows in this diagram are closed cofibrations and horizontal arrows on the right are Hurewitz fibrations. Kieboom's theorem [14] implies that the induced map $\mathcal{P}(B)=* \times_{B} B^{\mathrm{I}} \rightarrow \mathcal{P}(Y)=* \times_{Y} Y^{\mathrm{I}}$ is a closed cofibration. Applying finally the theorem of Kieboom to the diagram

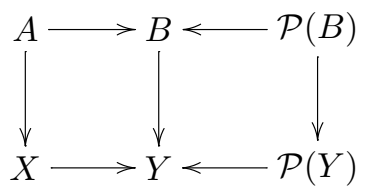

we conclude that the map $f i b(f)=A \times_{B} \mathcal{P}(B) \rightarrow f i b(g)=X \times_{Y} \mathcal{P}(Y)$ is also a closed cofibration.

A similar argument works for cofibers, only instead of Kieboom's theorem one should use this time the union theorem of Lillig [17].

COROLLARY E.3. - Let $f_{\bullet}: X_{\bullet} \rightarrow Y_{\bullet}$ be a morphism of good pointed simplicial spaces. Then the pointed simplicial spaces $f_{i b}\left(f_{\bullet}\right)$ and cofib $\left(f_{\bullet}\right)$ are also good.

\section{Acknowledgements}

The authors are both grateful for the hospitality of I.H.E.S. during the writing of this paper. Moreover, the first author thanks the I.A.S. and M.S.R.I., and the second author thanks M.P.I.Bonn for further hospitality. Finally, we thank the referee for his careful reading of our paper.

\section{REFERENCES}

[1] Bass H., Algebraic K-Theory, W.A. Benjamin, 1968.

[2] Beilinson A., Letter to C. Soulé (1982). 
[3] BLOCH S., Algebraic cycles and higher $K$-theory, Adv. in Math. 61 (1986) 267-304.

[4] BLoch S., The moving lemma for higher Chow groups, J. Algebraic Geom. 3 (1994) 537-568.

[5] Bloch S., Lichtenbaum S., A spectral sequence for motivic cohomology, Preprint.

[6] Bourbaki N., General Topology, Springer-Verlag, 1989.

[7] Bousfield A., Friedlander E., Homotopy theory of $\Gamma$-spaces, spectra, and bisimplicial sets, in: Geometric Applications of Homotopy Theory II, in: Lecture Notes in Math., Vol. 658, SpringerVerlag, 1978, pp. 80-130.

[8] BRowder W., Algebraic K-theory with coefficients $\mathbb{Z} / p$, in: Geometric Applications of Homotopy Theory, in: Lecture Notes in Math., Vol. 657, Springer-Verlag, 1978, pp. 40-84.

[9] Brown K., Gersten S., Algebraic K-theory as generalized sheaf cohomology, in: Algebraic K-theory, I: Higher K-theories, in: Lecture Notes in Math., Vol. 341, Springer-Verlag, 1973, pp. 266-292.

[10] Friedlander E., Voevodsky V., Bivariant cycle cohomology, in: Voevodsky V., Suslin A., Friedlander E. (Eds.), Cycles, Transfers and Motivic Homology Theories, in: Annals of Math. Studies, Vol. 143, 2000, pp. 138-187.

[11] Godement R., Topologie algébrique et théorie des faisceaux, Hermann, Paris, 1958.

[12] GRAYSON D., Weight filtrations via commuting automorphisms, K-Theory 9 (1995) 139-172.

[13] JARDINE J., Generalized étale cohomology theories, Birkhäuser Verlag, Basel, 1997.

[14] Кіевоом R., A pullback theorem for cofibrations, Manuscripta Math. 58 (1987) 381-384.

[15] LANDSBuRg S., Some filtrations on higher K-theory and related invariants, $K$-theory 6 (1992) 431457.

[16] LeVINE M., Techniques of localization in the theory of algebraic cycles, J. Algebraic Geom. 10 (2001) 299-363.

[17] LiLlig J., A union theorem for cofibrations, Arch. Math. 24 (1973) 410-415.

[18] Massey W., Products in exact couples, Ann. Math. 59 (1954) 558-569.

[19] Maclane S., Homology, Springer-Verlag, 1963.

[20] MAY P., Simplicial Objects in Algebraic Topology, University of Chicago Press, 1967.

[21] Morel F., Voevodsky V., $\mathbb{A}^{1}$-homotopy theory of schemes, Publ. Math. IHES 90 (2001) 45-143.

[22] OKa S., Multiplications on the Moore spectrum, Mem. Fac. Sci Kyushu, Ser. A 38 (1984) 257-276.

[23] PupPE D., Bemerkungen über die Erweiterung von Homotopien, Arch. Math. 18 (1967) 81-88.

[24] Quillen D., Homotopical Algebra, in: Lecture Notes in Math., Vol. 43, Springer-Verlag, 1967.

[25] Quillen D., Higher algebraic K-theory: I, in: Algebraic K-theory, I: Higher K-theories, in: Lecture Notes in Math., Vol. 341, Springer-Verlag, 1973, pp. 85-147.

[26] Segal G., Categories and cohomology theories, Topology 13 (1974) 293-312.

[27] Serre J.-P., Algèbre locale multiplicités, in: Lecture Notes in Math., Vol. 341, Springer-Verlag, 1975.

[28] Strom A., The homotopy category is a homotopy category, Arch. Math. 23 (1972) 435-441.

[29] Suslin A., Higher Chow groups and étale cohomology, in: Voevodsky V., Suslin A., Friedlander E. (Eds.), Cycles, Transfers and Motivic Homology Theories, in: Annals of Math. Studies, Vol. 143, 2000, pp. 237-252.

[30] Suslin A., Voevodsky V., Singular homology of abstract algebraic varieties, Invent. Math. 123 (1996) 61-94.

[31] SusLin A., Voevodsky V., Bloch-Kato conjecture and motivic cohomology with finite coefficients, in: The Arithmetic and Geometry of Algebraic Cycles (Banff, AB, 1998), in: NATO Sci. Ser. C Math. Phys. Sci., Vol. 548, 1998, pp. 117-189.

[32] Thomason R., Trobaugh T., Higher algebraic $K$-theory of schemes and of derived categories, in: The Grothendieck Festschrift, Vol. III, in: Progr. Math., Vol. 88, pp. 247-435.

[33] Voevodsky V., Cohomological theory of presheaves with transfers, in: Voevodsky V., Suslin A., Friedlander E. (Eds.), Cycles, Transfers and Motivic Homology Theories, in: Annals of Math. Studies, Vol. 143, 2000, pp. 87-137.

[34] Voevodsky V., Triangulated category of motives over a field, in: Voevodsky V., Suslin A., Friedlander E. (Eds.), Cycles, Transfers and Motivic Homology Theories, in: Annals of Math. Studies, Vol. 143, 2000, pp. 188-238.

[35] Voevods Ky V., Motivic cohomology are isomorphic to higher Chow groups, Preprint. 
[36] Voevodsky V., Mazza C., Weibel C., Lectures on motivic cohomology, Preprint.

[37] Waldhausen F., Algebraic K-theory of spaces, in: Algebraic and Geometric Topology (New Brunswick, NJ, 1983), in: Lecture Notes in Math., Vol. 1126, Springer-Verlag, 1985, pp. 318-419.

[38] Weibel C., An Introduction to Homological Algebra, Cambridge University Press, 1994.

[39] Weibel C., Products in higher Chow groups and motivic cohomology, in: Algebraic K-theory (Seattle, WA, 1997), in: Proceedings of Symposia in Pure Math., Vol. 67, 1999, pp. 305-315.

[40] WhiteHEAD G., Elements of Homotopy Theory, Springer-Verlag, 1978.

(Manuscrit reçu le 20 juillet 2000; accepté, après révision, le 10 mai 2002.)

Eric M. FRIEDLANDER and Andrei SUSLIN

Northwestern University,

Department of Mathematics,

Evanston, IL 60208, USA

E-mail: eric@math.nwu.edu (E.M. Friedlander),

suslin@dehn.math.nwu.edu (A. Suslin) 